\title{
Extended duality methods for nonlinear Helmholtz equations
}

\author{
Dissertation \\ zur Erlangung des Doktorgrades \\ der Naturwissenschaften
}

vorgelegt beim Fachbereich 12

der Johann Wolfgang Goethe-Universität

in Frankfurt am Main

von

Tolga Yeşil

aus Frankfurt am Main

Frankfurt am Main 2021 (D30) 
vom Fachbereich 12 der

Johann Wolfgang Goethe-Universität als Dissertation angenommen.

Dekan: Prof. Dr.-Ing. Lars Hedrich

Gutachter: Prof. Dr. Tobias Weth

Prof. Dr. Rupert L. Frank

Datum der Disputation: 16.4.2021 


\section{Contents}

$\begin{array}{ll}\text { Chapter 1. Overview } & 1\end{array}$

1. Introduction 3

2. Main results of this thesis 11

\begin{tabular}{ll}
\hline 3. Zusammenfassung & 21
\end{tabular}

Danksagungen 42

Chapter 2. Dual ground state solutions for the critical nonlinear Helmholtz equation 43

1. Introduction 43

2. The Helmholtz-resolvent in the Sobolev-critical case 48

3. Existence via the dual variational method 57

Chapter 3. Fourier-extension estimates and applications to a nonlinear Helmholtz equation 75

1. Introduction 75

2. Fourier extension estimates for $G_{k}$-invariant functions $\quad 82$

3. Resolvent estimates for $G$-invariant functions $\quad 88$

$4 . \quad$ Nonvanishing for $G$-invariant functions 93

\begin{tabular}{lll}
\hline $5 . \quad$ Dual variational framework and $G$-invariant solutions & 97
\end{tabular}

Chapter 4. Dual variational methods for a nonlinear Helmholtz equation with sign-changing nonlinearity 105

$\begin{array}{ll}1 . \text { Introduction } & 105\end{array}$

2. Dual variational formulation 108

$\begin{array}{lll}3 . & \text { Saddle-point reduction } & 110\end{array}$

4. Palais-Smale sequences for the reduced functional 113

\begin{tabular}{lll}
\hline 5. & Proof of Theorem 4.1 and Corollary 4.2 & 116
\end{tabular}

$\begin{array}{lll}\text { Chapter 5. Appendix } & 119\end{array}$

1. Failure of the nonvanishing-Theorem in the Stein-Tomas critical case 119

\begin{tabular}{lll}
\hline Chapter 6. Summary & 125
\end{tabular}

\begin{tabular}{ll}
\hline References constituting the authors dissertation thesis & 131
\end{tabular}

\begin{tabular}{ll}
\hline References & 133
\end{tabular} 
Dedicated to my grandparents Behice and Fevzi Yeşil 


\section{CHAPTER 1}

\section{Overview}

Dual variational methods have proven useful in a variety of situations in the context of nonlinear partial differential equations that did not allow an apparent direct variational approach. Probably one of the first works in this context is due to Clarke in [16, 17, where he introduces a "dual action principle" in order to find periodic solutions for Hamilton's equations by minimizing an integral that appears to be "dual" by means of the Fenchel-transform. Let us mention [18, where these ideas were carried out. See also [2], [3], [1], [19], [38, and [9] for more applications as well as recent examples where dual variational approaches have led to an advantageous and successful framework.

G. Evéquoz and T. Weth developed in [31] a dual variational framework for the nonlinear Helmholtz equation

$$
-\Delta u-k^{2} u=Q(x)|u|^{p-2} u, \quad x \in \mathbb{R}^{N},
$$

where $Q \in L^{\infty}\left(\mathbb{R}^{N}\right) \backslash\{0\}$ is non-negative, $k>0$ and $p \in\left[2_{*}, 2^{*}\right]$, with $2_{*}:=\frac{2(N+1)}{N-1}$ and $2^{*}:=\frac{2 N}{N-2}$ for space dimensions $N \geq 3$. In particular, the existence of standing-wave solutions, which arise from real-valued solutions, are obtained under additional requirements on $Q$ and p. Equation (1.1) is related to stationary Schrödinger equations of the type

$$
-\Delta u+\lambda u=f(x, u), \quad \lambda \in \mathbb{R}, \quad x \in \mathbb{R}^{N},
$$

which have been in the center of extensive research since the early 1980ies. Let us mention the works of Berestycki, Lions [7, 8, Ding and Ni [22], Floer and Weinstein [33] and many others in this context. Whereas the existence of real-valued solutions of (1.2) for $\lambda \geq 0$ is quite well understood (see e.g. [63, [69]), comparably less is known in the case $\lambda<0$, which corresponds to (1.1) by setting $\lambda=-k^{2}$ for the important case of power-type nonlinearities $f(x, u)=Q(x)|u|^{p-2} u$. One of the main reasons is the lack of a direct variational approach, since by classical results of Rellich [56] and Kato [40], solutions of the Helmholtz equation decay at most like

$$
u(x)=O\left(|x|^{\frac{1-N}{2}}\right), \quad \text { as }|x| \rightarrow \infty,
$$

thus are only to be expected to be in $L^{p}\left(\mathbb{R}^{N}\right)$ (or $\left.W^{2, p}\left(\mathbb{R}^{N}\right)\right)$ for values $p>\frac{2 N}{N-1}$. Therefore, $H^{1}$-solutions will not exists in general, as would be required for the natural energy functional associated with (1.1), and powerful tools as the concentration compactness machinery of Lions 
[47, [48, 49, ,50] are not available in this case. Here $W^{m, p}\left(\mathbb{R}^{N}\right)$ denotes the usual Sobolevspace with weak derivatives up to order $m \in \mathbb{N}$ that belong to $L^{p}\left(\mathbb{R}^{N}\right)$ and $H^{m}\left(\mathbb{R}^{N}\right):=$ $W^{m, 2}\left(\mathbb{R}^{N}\right)$. Additionally, in the situation of (1.1) we have, that 0 is contained in the essential spectrum of the (linearized) Schrödinger operator $-\Delta-k^{2}$ which limits the applicability of functional analytic tools.

In the present thesis we focus on extensions of the dual methods developed in [31. In particular, we will be concerned with the Sobolev-critical case $p=2^{*}$ (Chapter 2), the possibility to extend the dual framework for values $2<p \leq 2_{*}$ (Chapter 3) and describe an extension of the dual framework, that allows sign-changing weight functions (Chapter 4). In all of these cases we will in particular establish the existence of real-valued solutions of (1.1) under these new requirements.

Let us give an overview regarding the organization of this thesis. We start by introducing the Helmholtz equation in the context of wave propagation and recall the linear case briefly. Then we introduce the Helmholtz resolvent operator, fundamental solutions and recall important results concerning boundedness properties that will be of great significance throughout the text. We then recall one of the first results for the nonlinear problem (1.1] due to Gutièrrez [37] and formulate the basics concepts within her approach, also to relate the upcoming strategies and results. Equipped with these preliminary results, we are able formulate the dual variational framework developed in [31, present the main results, discuss certain limitations and recall further results established in this context. This allows us to present and classify the established results in [Y1], [Y2], [Y3], which we will then present in the Chapters 2,3 and 4 respectively. The results presented in Chapter 2 result from a collaboration with Gilles Evèquoz published in [Y1]. The content of Chapter 3 is a joint work with Tobias Weth published in [Y2]. The results in Chapter 4 can be found in [Y3] and are a result of a collaboration with Rainer Mandel and Dominic Scheider.

These will be presented in the same form as they can be found in the published versions. Additional details will be provided in the corresponding prefaces at the beginning of chapters 2,3 and 4 respectively. 


\section{Introduction}

Real-valued solutions of the nonlinear Helmholtz equation arise in the study of high-frequency time-harmonic solutions of the nonlinear Klein-Gordon equation

$$
\frac{\partial^{2} \psi}{\partial t^{2}}-\Delta \psi(t, x)+m \psi(t, x)=f(x, \psi(t, x)), \quad m>0, \quad(t, x) \in \mathbb{R} \times \mathbb{R}^{N},
$$

if the nonlinearity has the form $f(x, \psi)=g\left(x,|\psi|^{2}\right) \psi$. This can be seen via the time-harmonic ansatz $\psi(t, x)=e^{\mathrm{i} k t} u(x)$ for which (1.4) leads to

$$
-\Delta u+\left(m-\omega^{2}\right) u=f(x, u), \quad x \in \mathbb{R}^{N} .
$$

This equation has the form (1.2) with $\lambda=m-\omega^{2}$; thus we have $\lambda<0$ in the case of high frequencies $\omega^{2}>m$. The Klein-Gordon equation (1.4) is a central tool in the mathematical description of wave propagation in an ambient medium with nonlinear response, which appears to be a fascinating and challenging field in nonlinear analysis (see e.g. [42] and [61 for a detailed description). Thus, real valued solutions of Helmholtz equations help to investigate this important class of (high-frequency) time-periodic solutions as an essential step towards a full description and deeper understanding for wave phenomena.

1.1. Fundamental solution and resolvent operator. The decay condition (1.3) for solutions of the Helmholtz equation is incomplete from an analytical point of view, as it is necessary to distinguish the radiative behavior of the solutions at infinity (c.f. [55, Chapter $2])$. In the context of the linear problem

$$
-\Delta u-k^{2} u=f(x), \quad x \in \mathbb{R}^{N}
$$

for some (possibly complex-valued) function $f$ on $\mathbb{R}^{N}$, it was suggested by Sommerfeld (see [58] ) to additionally introduce the outgoing radiation condition

$$
r^{\frac{N-1}{2}}\left|\frac{\partial u}{\partial r}-\mathrm{i} k u\right| \rightarrow 0, \quad \text { as } r=|x| \rightarrow \infty .
$$

The corresponding solutions of the linear equation (1.6) represent outgoing waves that are stimulated by the source $f$. If $f$ is sufficiently benign (e.g. continuous and compactly supported), then (1.6), (1.7) has a unique solution (formally) given by $u=\left(-\Delta-k^{2}\right)^{-1}(f)$ (see [20]). Let us give a brief description how this resolvent operator can be derived. For $\varepsilon>0$ and $k>0$ the operator $-\Delta-\left(k^{2}+\mathrm{i} \varepsilon\right): H^{2}\left(\mathbb{R}^{N}\right) \subset L^{2}\left(\mathbb{R}^{N}\right) \rightarrow L^{2}\left(\mathbb{R}^{N}\right)$ is an isomorphism. For any Schwartz-function $f \in \mathcal{S}\left(\mathbb{R}^{N}\right)$ its inverse is given by

$$
\mathcal{R}_{k, \varepsilon} f(x):=\left[-\Delta-\left(k^{2}+\mathrm{i} \varepsilon\right)\right]^{-1} f(x)=(2 \pi)^{\frac{N}{2}} \int_{\mathbb{R}^{N}} e^{\mathrm{i} x \cdot \xi} \frac{\widehat{f}(\xi)}{|\xi|^{2}-\left(k^{2}+\mathrm{i} \varepsilon\right)} d \xi
$$

where

$$
\mathcal{F}(f)(\xi)=\widehat{f}(\xi):=(2 \pi)^{-\frac{N}{2}} \int_{\mathbb{R}^{N}} e^{\mathrm{i} x \cdot \xi} f(x) d x
$$


denotes the Fourier transform of $f: \mathbb{R}^{N} \rightarrow \mathbb{C}$. As described in [36], there exists a linear operator $\mathcal{R}_{k}: \mathcal{S}\left(\mathbb{R}^{N}\right) \rightarrow \mathcal{S}^{\prime}\left(\mathbb{R}^{N}\right)$ such that

$$
\left\langle\mathcal{R}_{k} f, g\right\rangle=\lim _{\varepsilon \rightarrow 0} \int_{\mathbb{R}^{N}}\left[\mathcal{R}_{k, \varepsilon} f\right](x) g(x) d x=\int_{\mathbb{R}^{N}}\left[\Phi_{k} * f\right](x) g(x) d x, \quad \text { for } f, g \in \mathcal{S}\left(\mathbb{R}^{N}\right)
$$

with $\Phi_{k}: \mathbb{R}^{N} \backslash\{0\} \rightarrow \mathbb{C}$ given by

$$
\Phi_{k}(x)=\lim _{\varepsilon \rightarrow 0}(2 \pi)^{-\frac{N}{2}} \mathcal{F}^{-1}\left(\left(|\cdot|^{2}-k^{2}-\mathrm{i} \varepsilon\right)^{-1}\right)(x)=\frac{\mathrm{i}}{4}\left(\frac{k}{2 \pi|x|}\right)^{\frac{N-2}{2}} H_{\frac{N-2}{2}}^{(1)}(k|x|) .
$$

The function $\Phi_{k}$ is the fundamental solution to (1.6) satisfying Sommerfeld's outgoing radiation condition 1.7 and $H_{\frac{N-2}{2}}^{(1)}$ denotes the Hankel-function of the first kind of order $\frac{N-2}{2}$ (see [68] for a detailed treatment of Hankel-functions).

Boundedness properties of the resolvent operator $\mathcal{R}_{k}$ turn out to be crucial for the study of the nonlinear problem (1.1). A first key result is the following estimate by C. E. Kenig, A. Ruiz and C.D. Sogge.

Theorem 1.1. [43, Special case of Theorem 2.3] Let $p \in\left[2_{*}, 2^{*}\right]$. Then there exists a constant $C=C(N, p)>0$ such that

$$
\left\|\mathcal{R}_{k} f\right\|_{L^{p}\left(\mathbb{R}^{N}\right)} \leq C k^{(N-2)-\frac{2 N}{p}}\|f\|_{L^{p^{\prime}}\left(\mathbb{R}^{N}\right)} \quad \text { for all } f \in \mathcal{S}\left(\mathbb{R}^{N}\right) .
$$

Here and in the following $p^{\prime}:=\frac{p}{p-1}$ denotes the Hölder-conjugate to $p \in(1, \infty)$.

Thus, the operator $\mathcal{R}_{k}$ can be continuously extended as a linear map $\mathcal{R}_{k}: L^{p^{\prime}}\left(\mathbb{R}^{N}\right) \rightarrow L^{p}\left(\mathbb{R}^{N}\right)$ such that $(1.9)$ holds for all $f \in L^{p^{\prime}}\left(\mathbb{R}^{N}\right)$. Let us also mention the following non-selfdual generalization of Theorem 1.1 from [37.

Theorem 1.2. Let $t \in\left[1, \frac{2 N}{N+1}\right)$ and $q \in\left(\frac{2 N}{N-1}, \infty\right]$ satisfy $\frac{2}{N+1} \leq \frac{1}{t}-\frac{1}{q} \leq \frac{2}{N}$ if $N \geq 3$. Then there exists a constant $C=C(N, t, q)$ such that

$$
\left\|\mathcal{R}_{k}\right\|_{L^{q}\left(\mathbb{R}^{N}\right)} \leq C k^{N(1 / t-1 / q-2 / N)}\|f\|_{L^{t}\left(\mathbb{R}^{N}\right)} \quad \text { for all } f \in \mathcal{S}\left(\mathbb{R}^{N}\right) .
$$

We see that Theorem 1.1 is implied by Theorem 1.2 with $q=p$ and $t=p^{\prime}$.

The given range for $t, q$ in Theorem 1.2 and thus for $p$ in Theorem 1.1 is optimal as can be seen by the counterexample of Börjeson [12, pp. 231-233]. Concerning the proof of Theorems 1.1 and 1.2 let us mention that the given limitation regarding the upper bound, namely $1 / t-1 / q \leq 2 / N$ (and $p \leq 2^{*}$ in the selfdual case, respectively) is due to an underlying Hardy-Littlewood-Sobolev inequality (c.f. [46, Theorem 4.3]) and we will refer this case in the following as the Sobolevcritical case. The lower critical case comes from the Stein-Tomas inequality (see Theorem 1.3 and 1.4 below), so that we will refer to this case in the following as the Stein-Tomas critical case. The Stein-Tomas inequality analyses boundedness properties of Fourier-restriction or Fourier-extension operators. For $F \in L^{2}\left(\mathbb{S}^{N-1}\right)$, where $\mathbb{S}^{N-1}:=\left\{x \in \mathbb{R}^{N}:|x|=1\right\}$ denotes 
the unit sphere in $\mathbb{R}^{N}$, the (inverse) Fourier-extension of $F$ is given by

$$
\check{F}_{\sigma}(x):=(2 \pi)^{-\frac{N}{2}} \int_{\mathbb{S}^{N-1}} e^{\mathrm{i} x \cdot \omega} F(\omega) d \sigma(\omega),
$$

where $d \sigma$ denotes the induced Lebesgue measure on $\mathbb{S}^{N-1}$. Let us state both (equivalent) versions of the Stein-Tomas results (see also [59, §8 Corollary 5.4]).

Theorem 1.3 (Stein-Tomas extension-theorem). Let $F \in L^{2}\left(\mathbb{S}^{N-1}\right)$. Then $\check{F}_{\sigma}$ belongs to $L^{q}\left(\mathbb{R}^{N}\right)$ for all $q \geq 2$ and we have

$$
\left\|\check{F}_{\sigma}\right\|_{L^{q}\left(\mathbb{R}^{N}\right)} \leq C\|F\|_{L^{2}\left(\mathbb{S}^{N-1}\right)}
$$

with a constant $C>0$ independent of $F$.

ThEOREM 1.4 (Stein-Tomas restriction-theorem). Let $1 \leq q \leq\left(2_{*}\right)^{\prime}$ and $f \in L^{q}\left(\mathbb{R}^{N}\right)$. Then for the Fourier transform $\widehat{f}$ we have $\widehat{f}_{\mathbb{S}^{N-1}} \in L^{2}\left(\mathbb{S}^{N-1}\right)$ and there exists a constant $C>0$ independent of $f$, such that

$$
\int_{\mathbb{S}^{N-1}}|\widehat{f}(\omega)|^{2} d \sigma(\omega) \leq C\|f\|_{L^{q}\left(\mathbb{R}^{N}\right)} .
$$

As can be seen by the famous Knapp example (c.f. [66, §4]) the range of values regarding $q$ in (1.11) and (1.12) is sharp. However, for radial (and thus constant) functions $F \in L^{2}\left(\mathbb{S}^{N-1}\right)$ the estimate (1.11) can be seen to be true (c.f. [59, $\S 8$ Proposition 5.1]) for all $q>\frac{2 N}{N-1}$. Contrary to the proof of Theorem 1.3 (or Theorem 1.4) this particular result can be achieved mainly by using the key bound

$$
d \check{\sigma}_{N}(x) \leq C(1+|x|)^{\frac{1-N}{2}}, \quad x \in \mathbb{R}^{N},
$$

where $d \check{\sigma}_{N}:=\check{F}_{\sigma}$ for $F \equiv 1$. Stein-Tomas type results have been receiving extensive attention due to their various applications and consequences to several fields in mathematics (see also [34, 66]). Variants of the Stein-Tomas Theorem play a crucial role in order to obtain Strichartz-estimates for the Wave- or Schrödinger equation (see [13], [41] 62]).

1.2. Small complex solutions for the nonlinear equation. One of the very first existence results for a nonlinear Helmholtz equation of the type (1.1) is due to Gutiérrez [37]. She proves the existence of (small) complex solutions for $N=3,4, p=4$ and $Q \equiv \pm 1$, that additionally satisfy an integral variant of the decay condition $(1.3)$ given by

$$
\sup _{R>1} \frac{1}{R} \int_{B_{R}(0)}|u|^{2} d x<\infty .
$$

A key observation in her approach is that for $N \geq 3$ and $p \in\left[2_{*}, 2^{*}\right]$ a (complex-valued) function $u \in W^{2, p}\left(\mathbb{R}^{N}\right)$ is a solution of (1.1) satisfying (1.14) if and only if $u \in L^{p}\left(\mathbb{R}^{N}\right)$ solves 
the integral equation

$$
u=\mathcal{R}_{k}\left(Q|u|^{p-2} u\right)+\varphi_{g}
$$

for some $\varphi_{g} \in \mathcal{H}$. Here $\mathcal{H}$ denotes the space of Herglotz-wave functions given by

$$
\mathcal{H}:=\left\{\varphi_{g}: \mathbb{R}^{N} \rightarrow \mathbb{C}: \varphi_{g}(x)=\int_{\mathbb{S}^{N-1}} e^{\mathrm{i} k x \cdot \xi} g(\omega) d \sigma(\omega) \text { for some } g \in L^{2}\left(\mathbb{S}^{N-1}\right)\right\} .
$$

Thus we have with Theorem 1.3 that every $\varphi_{g}=(2 \pi)^{\frac{N}{2}} \check{g}_{\sigma} \in L^{q}\left(\mathbb{R}^{N}\right)$ for all $q \geq 2_{*}$. Every $\varphi_{g} \in \mathcal{H}$ is a solution of the linear equation $(1.6)$ with $f \equiv 0$ (see [37, chapter 2]).

The nonlinearity in her result has the explicit form $f(x, u)=Q(x)|u|^{2} u$ and thus defines a substitution operator $N_{f}: L^{p}\left(\mathbb{R}^{N}\right) \rightarrow L^{p^{\prime}}\left(\mathbb{R}^{N}\right)$ for $p=4$. Consequently, the integral equation (1.15) reduces to the fixed-point problem

$$
u=\mathcal{R}_{k} N_{f}(u)+\varphi_{g}, \quad \text { for some } \varphi_{g} \in \mathcal{H},
$$

which is then solved with non-selfdual resolvent estimates and contraction-mapping techniques.

1.3. Dual variational framework for the nonlinear Helmholtz equation. Evèquoz and Weth [31] focus on the the complementary class of real-valued solutions for (1.1). Assuming that $Q \in L^{\infty}\left(\mathbb{R}^{N}\right) \backslash\{0\}$ is non-negative and $p \in\left[2_{*}, 2^{*}\right]$, they observe that real-valued solutions can be obtained as solutions of the integral equation

$$
u=\mathbf{R}_{k}\left(Q|u|^{p-2} u\right), \quad u \in L^{p}\left(\mathbb{R}^{N}\right) .
$$

Here $\mathbf{R}_{k}$ denotes the real part of $\mathcal{R}_{k}$ given by $\mathbf{R}_{k}(f)=\Psi_{k} * f$ and

$$
\Psi_{k}(x):=\operatorname{Re}\left(\Phi_{k}\right)(x)=-\frac{1}{4}\left(\frac{k}{2 \pi|x|}\right)^{\frac{N-2}{2}} Y_{\frac{N-2}{2}}(k|x|), \quad x \in \mathbb{R}^{N} \backslash\{0\},
$$

where $Y_{\frac{N-2}{2}}$ is the Bessel-function of the second kind of order $\frac{N-2}{2}$ (see [68 for a detailed study of these functions). A first central observation is that solutions of (1.17) satisfy (1.15) with $g=g_{u}$ given by

$$
g_{u}(\xi)=-\frac{\mathrm{i}}{4}\left(\frac{k^{2}}{2 \pi}\right)^{\frac{N-2}{2}} \mathcal{F}\left(Q|u|^{p-2} u\right)(k \xi), \quad \xi \in \mathbb{S}^{N-1} .
$$

Therefore, a solution $u$ is nonlinearly related to the associated Herglotz-wave, which (see e.g. [31]) is indeed uniquely determined.

Notice, that by (1.9) the trivial solution $u \equiv 0$ is an isolated solution of (1.17), thus contraction mapping arguments will not lead to nontrivial solutions of this problem. Instead, the idea is to introduce a dual transformation leading to a new problem that appears to have a rich variational structure. To this purpose let us assume $k=1$ to simplify the notation. The 
general case can be recovered by scaling. We are thus led to study the equation

$$
-\Delta u-u=Q(x)|u|^{p-2} u, \quad x \in \mathbb{R}^{N}
$$

and the corresponding integral equation

$$
u=\mathbf{R}\left(Q|u|^{p-2} u\right), \quad u \in L^{p}\left(\mathbb{R}^{N}\right)
$$

with $\mathbf{R}:=\mathbf{R}_{1}$.

REMARK 1.5. According to [31, Lemma 4.3] for $p \in\left[2_{*}, 2^{*}\right]$ any solution $u \in L^{p}\left(\mathbb{R}^{N}\right)$ of $u=\mathbf{R}\left(Q|u|^{p-2}\right)$ for $Q \in L^{\infty}\left(\mathbb{R}^{N}\right)$ is indeed a strong solution $u \in W^{2, q}\left(\mathbb{R}^{N}\right) \cap \mathcal{C}^{1, \gamma}\left(\mathbb{R}^{N}\right)$ for all $q \in[p, \infty)$ and $\gamma \in(0,1)$ of $(1.20)$. Using the same arguments as in [51, p.13] this can be improved to $W^{2, q}\left(\mathbb{R}^{N}\right) \cap \mathcal{C}^{1, \gamma}\left(\mathbb{R}^{N}\right)$ for all $q \in\left(\frac{2 N}{N-1}, \infty\right), \gamma \in(0,1)$.

Any solution of (1.21) will be referred as a dual bound state solution.

By setting $v:=Q^{\frac{1}{p^{\prime}}}|u|^{p-2} u \in L^{p^{\prime}}\left(\mathbb{R}^{N}\right)$ for $u \in L^{p}\left(\mathbb{R}^{N}\right)$ the integral equation (1.21) leads to

$$
|v|^{p^{\prime}-2} v=Q^{\frac{1}{p}} \mathbf{R}\left(Q^{\frac{1}{p}} v\right):=\mathbf{K}_{Q, p}(v) .
$$

The problem 1.22 is variational and the associated (dual) energy functional $J_{Q, p}: L^{p^{\prime}}\left(\mathbb{R}^{N}\right) \rightarrow$ $L^{p}\left(\mathbb{R}^{N}\right)$ is given by

$$
J_{Q, p}(v)=\frac{1}{p^{\prime}}\|v\|_{L^{p^{\prime}\left(\mathbb{R}^{N}\right)}}^{p^{\prime}}-\frac{1}{2} \int_{\mathbb{R}^{N}} v \mathbf{K}_{Q, p}(v) d x
$$

The functional $J_{Q, p}$ is of class $\mathcal{C}^{1}$ with derivative

$$
J_{Q, p}^{\prime}(v) w=\int_{\mathbb{R}^{N}}\left(|v|^{p^{\prime}-2} v-\mathbf{K}_{Q, p}(v)\right) w d x, \quad \text { for all } v, w \in L^{p^{\prime}}\left(\mathbb{R}^{N}\right)
$$

and possesses the Mountain-Pass geometry as summarized in the following lemma.

Lemma 1.6. [31, Lemma 4.2] Let $p \in\left[2_{*}, 2^{*}\right]$ and consider $Q \in L^{\infty}\left(\mathbb{R}^{N}\right) \backslash\{0\}$ such that $Q \geq 0$ a.e. on $\mathbb{R}^{N}$.

(i) There exists $\delta>0$ and $\rho \in(0,1)$ such that $J_{Q, p}(v) \geq \delta>0$ for all $v \in L^{p^{\prime}}\left(\mathbb{R}^{N}\right)$ with $\|v\|_{L^{p^{\prime}}\left(\mathbb{R}^{N}\right)}=\rho$.

(ii) There is $v_{0} \in L^{p^{\prime}}\left(\mathbb{R}^{N}\right)$ such that $\left\|v_{0}\right\|_{L^{p^{\prime}\left(\mathbb{R}^{N}\right)}}>1$ and $J_{Q, p}\left(v_{0}\right)<0$.

(iii) Every Palais-Smale sequence for $J_{Q, p}$ is bounded in $L^{p^{\prime}}\left(\mathbb{R}^{N}\right)$.

Let us recall that a sequence $\left(v_{n}\right) \subset L^{p^{\prime}}\left(\mathbb{R}^{N}\right)$ is called a Palais-Smale-sequence ((PS)-sequence), if $\left(J_{Q}\left(v_{n}\right)\right)_{n} \subset \mathbb{R}$ is bounded and $\left\|J_{Q, p}^{\prime}\left(v_{n}\right)\right\|_{*} \rightarrow 0$ as $n \rightarrow \infty$, where the latter denotes the operatornorm of $J_{Q, p}^{\prime}\left(v_{n}\right)$. If in addition $J_{Q, p}\left(v_{n}\right) \rightarrow c \in \mathbb{R}$ as $n \rightarrow \infty$, then $\left(v_{n}\right)_{n} \subset L^{p^{\prime}}\left(\mathbb{R}^{N}\right)$ is called a $(P S)_{c}$-sequence. Moreover, $J_{Q, p}$ is said to satisfy the Palais-Smale-condition at level

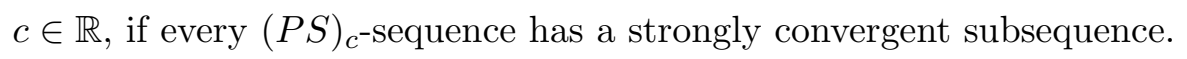


Compactness properties of $J_{Q, p}$ are crucially linked to compactness properties of the BirmanSchwinger operator $\mathbf{K}_{Q, p}$, which in turn are closely related to decay properties of the weightfunction $Q$ and the choice of $p$. In fact, we want to point out the following result.

Lemma 1.7. [31, Lemma 4.1] Let $p \in\left[2 *, 2^{*}\right)$ and consider $Q \in L^{\infty}\left(\mathbb{R}^{N}\right)$ with $Q \geq 0$ a.e. on $\mathbb{R}^{N}$. Then the operator

$$
\mathbf{K}_{Q, p}: L^{p^{\prime}}\left(\mathbb{R}^{N}\right) \rightarrow L^{p}\left(\mathbb{R}^{N}\right), \quad \mathbf{K}_{Q, p}(v):=Q^{\frac{1}{p}} \mathbf{R}\left(Q^{\frac{1}{p}} v\right)
$$

is symmetric in the sense of

$$
\int_{\mathbb{R}^{N}} v \mathbf{K}_{Q, p}(w) d x=\int_{\mathbb{R}^{N}} w \mathbf{K}_{Q, p}(v) d x, \quad \text { for all } v, w \in L^{p^{\prime}}\left(\mathbb{R}^{N}\right) .
$$

Moreover,

(i) $\mathbf{K}_{Q, p}$ is locally compact, i.e. for any bounded and measurable set $B \subset \mathbb{R}^{N}$, the operator $\mathbb{1}_{B} \mathbf{K}_{Q, p}$ is compact. Here $\mathbb{1}_{B}$ denotes to characteristic function of a measurable set $B \subset$ $\mathbb{R}^{N}$.

(ii) if $\operatorname{esssup}_{|x| \geq \rho} Q(x) \rightarrow 0$ as $\rho \rightarrow \infty$, then $\mathbf{K}_{Q, p}$ itself is compact.

If $Q$ vanishes at infinity, then from Lemma 1.7 (ii) it can be deduced, that the functional $J_{Q, p}$ satisfies the Palais-Smale condition at every level. A symmetric Mountain-Pass theorem then gives the existence of infinitely many pairs $\pm u$ of critical points for $J_{Q, p}$. Combining this existence and multiplicity result with a nonlinear far-field relation, the full result from [31] concerning this case reads as follows:

Theorem 1.8. [31, Theorem 1.2] Let $N \geq 3, p \in\left[2 *, 2^{*}\right)$ and let $Q \in L^{\infty}\left(\mathbb{R}^{N}\right) \backslash\{0\}$ be nonnegative with $\operatorname{essup}_{|x| \geq \rho} Q(x) \rightarrow 0$ as $\rho \rightarrow \infty$. Then $(1.20$ admits an unbounded sequence of pairs $\pm u_{n}$ of dual bound state solutions. Moreover, all of these solutions satisfy the nonlinear far field relation

$$
\lim _{R \rightarrow \infty} \frac{1}{R} \int_{B_{R}(0)}\left|u_{n}(x)+2\left(\frac{2 \pi}{|x|}\right)^{\frac{N-1}{2}} \operatorname{Re}\left(e^{i|x|-\frac{i(N-1) \pi}{4}}\right) g_{u_{n}}\left(\frac{x}{|x|}\right)\right|^{2} d x
$$

with $g_{u_{n}}$ given as in 1.19

Thus, taking Remark 1.5 into account, we indeed obtain strong solutions of 1.20 .

A second situation, where the existence of dual bound state solutions is obtained in [31], is that of periodic $Q$. In this case, the functional $J_{Q, p}$ will not satisfy the Palais-Smale condition in general, but the existence of a bounded Palais-Smale sequence at the Mountain-Pass level

$$
c:=\inf _{\gamma \in \Gamma} \sup _{t \in[0,1]} J_{Q, p}(\gamma(t)),
$$

where $\Gamma:=\left\{\gamma \in \mathcal{C}\left([0,1], L^{p^{\prime}}\left(\mathbb{R}^{N}\right)\right): \gamma(0)=0, J(\gamma(1))<0\right\}$, can still be shown (see e.g. [31, Lemma 6.1]). Note that by Lemma 1.6 we have $\Gamma \neq \emptyset$ and $c>0$. This allows to deduce the 
existence of a critical point by an approximation argument, but its non-triviality is unclear. A key component in this context is the following nonvanishing result:

TheOREM 1.9. [31, Theorem 3.1] Let $N \geq 3$, and $p \in\left(2_{*}, 2^{*}\right)$. Moreover let $\left(u_{n}\right)_{n} \subset L^{p^{\prime}}\left(\mathbb{R}^{N}\right)$ be a bounded sequence satisfying

$$
\limsup _{n \rightarrow \infty}\left|\int_{\mathbb{R}^{N}} u_{n} \mathcal{R}\left(u_{n}\right) d x\right|>0 .
$$

Then there exist $R>0, \zeta>0$ and a sequence $\left(x_{n}\right)_{n} \subset \mathbb{R}^{N}$ such that, up to a subsequence,

$$
\int_{B_{R}\left(x_{n}\right)}\left|u_{n}\right|^{p^{\prime}} d x \geq \zeta>0 \quad \text { for all } n \text {. }
$$

Here we have abbreviated $\mathcal{R}:=\mathcal{R}_{1}$ for the full resolvent. Let us say a few words to the proof, since we will be concerned with various extensions of this result. The proof of Theorem 1.9 relies on a decomposition of the fundamental solution $\Phi:=\Phi_{1}$ given in $(1.8)$ similar to [37. Thus we introduce $\Phi=\Phi_{(1)}+\Phi_{(2)}$ with

$$
\Phi_{(1)}:=\eta * \Phi, \quad \Phi_{(2)}:=\Phi-\Phi_{(1)}
$$

for $\eta \in \mathcal{S}\left(\mathbb{R}^{N}\right)$ such that $\widehat{\eta} \in \mathcal{C}_{c}^{\infty}\left(\mathbb{R}^{N}\right)$ with values in $[0,1], \widehat{\eta}=1$ on $\left\{|| \xi|-1| \leq \frac{1}{6}\right\}$ and $\widehat{\eta}=0$ on $\left\{|| \xi \mid-1 \geq \frac{1}{4}\right\}$. From the asymptotics of Hankel-functions we deduce that

$$
|\Phi(x)| \leq C_{0} \max \left\{|x|^{2-N},|x|^{\frac{1-N}{2}}\right\}, \quad \text { for } x \in \mathbb{R}^{N} \backslash\{0\},
$$

for $C_{0}>0$ and arguing as in the remark after [31, Theorem 3.1] one deduces that

$$
\left|\Phi_{(1)}(x)\right| \leq C_{0}(1+|x|)^{\frac{1-N}{2}}, \quad x \in \mathbb{R}^{N}
$$

and

$$
\left|\Phi_{(2)}(x)\right| \leq C_{0} \min \left\{|x|^{2-N},|x|^{-N}\right\}, \quad \text { for } x \in \mathbb{R}^{N} \backslash\{0\} .
$$

Theorem 1.9 is then proved for $\Phi_{(1)}, \Phi_{(2)}$ separately. The result concerning $\Phi_{(2)}$ is comparably short and causes the limitation $p<2^{*}$. For $\Phi_{(1)}$ one important step is to estimate $\left\|\mathbb{1}_{M_{R}} \Phi_{(2)}\right\|_{L^{p}\left(\mathbb{R}^{N}\right)}$ for $M_{R}:=\mathbb{R}^{N} \backslash B_{R}$, where $B_{R}$ is the (open) ball with Radius $R$ around the origin, by decomposing dyadicly for some $R>0$. Then each dyadic summand satisfies a straight forward $\left(L^{\infty}, L^{1}\right)$-bound. With the help of the Stein-Tomas Theorem 1.4 , then an $\left(L^{2}, L^{(2 *)^{\prime}}\right)$-bound is derived. Complex interpolation yields a selfdual bound for each dyadic summand of $\left\|\mathbb{1}_{M_{R}} \Phi_{(2)}\right\|_{L^{p}\left(\mathbb{R}^{N}\right)}$ with a prefactor $R^{A_{j}}$ where $j \in \mathbb{N}$ is the index of the summand and $A_{j}:=j A(N, p)$ is resulting from the interpolation procedure and has the property that $A(N, p)<0$ if and only if $p>2_{*}$, which is necessary for convergence of the associated dyadic series. Thus, the proof can only be established for $p>2_{*}$. 
We shall see, that the statement of Theorem 1.9 continues to hold in the Sobolev-critical case $p=2^{*}$ using a modification of the argument concerning $\Phi_{(2)}$ (see Theorem 2.6 below) but indeed fails to hold in the Stein-Tomas critical case $p=2_{*}$ as will be demonstrated in Chapter 5 below.

Let us remark, that for a bounded Palais-Smale sequence $\left(u_{n}\right)_{n} \subset L^{p^{\prime}}\left(\mathbb{R}^{N}\right)$ at the MountainPass level $c>0$ we have

$$
\int_{\mathbb{R}^{N}} Q^{\frac{1}{p}} u_{n} \mathbf{R}\left(Q^{\frac{1}{p}} u_{n}\right) d x=\frac{2 p^{\prime}}{2-p^{\prime}}\left(J_{Q, p}\left(u_{n}\right)-\frac{1}{p^{\prime}} J_{Q, p}^{\prime}\left(u_{n}\right) u_{n}\right) \rightarrow \frac{2 p^{\prime}}{2-p^{\prime}} c>0
$$

as $n \rightarrow \infty$, thus $\left(u_{n}\right)_{n} \subset L^{p^{\prime}}\left(\mathbb{R}^{N}\right)$ satisfies the conditions of Theorem 1.9 . The proof of the non-triviality of the critical point is then obtained with a local-convergence argument in the corresponding dual formulation (see e.g. [31, Theorem 6.2]). In total, the following result is stated in the case of periodic $Q$ :

Theorem 1.10. [31, Theorem 1.1] Let $N \geq 3, p \in\left(2_{*}, 2^{*}\right)$ and let $Q \in L^{\infty}\left(\mathbb{R}^{N}\right) \backslash\{0\}$ be non-negative and $\mathbb{Z}^{N}$-periodic. Then 1.20 admits a nontrivial dual bound state solution $u$ that additionally satisfies 1.25 with $g_{u}$ in place of $g_{u_{n}}$.

1.4. Further and related results. In the case of spatially compactly supported superlinear nonlinearities $f$, in [30] existence and multiplicity results are obtained for the problem

$$
-\Delta u-k^{2} u=f(x, u), \quad x \in \mathbb{R}^{N} .
$$

By using a Dirichlet-to-Neumann map associated to the exterior problem for the corresponding linear equation, the problem is reduced to the existence of critical points for an $H^{1}$-functional defined on a ball centered at the origin. These methods are heavily based on the compact support (in space) of the nonlinearity $f$. In [27] a dual approach in Orlicz spaces is developed that allows to treat more general nonlinearities than the model case $f(x, u)=Q|u|^{p-2} u$. In particular, under additional decay assumptions on the superlinear and subcritical nonlinearity, multiplicity results as well as far-field relations are derived for the obtained real valued solutions. In [29] a multiplicity result concerning the case of periodic weight functions $Q$ is proved, where ideas for a periodic Schrödinger equation developed in [64] have been successfully integrated to the dual context. Moreover, the existence of real valued solutions in the noncritical case $p \in\left(2_{*}, 2^{*}\right)$ for $(1.1)$ is established for asymptotically periodic weight functions, i.e. weight functions of the form $Q=Q_{0}+Q_{p e r}$, wher $Q_{0}$ vanishes at infinity and $Q_{\text {per }}$ is $\mathbb{Z}^{N}$-periodic.

Let us mention that the dual variational framework was elaborated in [52] for the defocusing case $Q \leq 0$. Moreover, the authors obtain radial solutions of 1.30 for a broad class of radial nonlinearites allowing values $p>\frac{2 N}{N-1}$. In 32 equation 1.1 is considered under the viewpoint of the existence of continuous solutions branches depending on the parameter $\lambda=-k^{2}$ in the spirit of $[\mathbf{8}, \mathbf{7}]$, for values of $\lambda$ that may enter the essential spectrum of $-\Delta$. The existence and behavior of standing-wave solutions for 11.1 in the plane is obtained in [26]. The existence 
and properties of high-wave number solutions for (1.1) was analyzed in [28. In [15] complex solutions for the Helmholtz equation with general nonlinearity are provided. The authors overcome the lack of maximum principles and the dual variational framework by analyzing the integral equation (4.11) with the help of topological fixed-point theory and global bifurcation. Let us further mention the articles [21, [53, [54], where the dual approach is extended to Helmholtz systems.

\section{Main results of this thesis}

In the following we will summarize each of the articles [Y1], $\mathbf{Y 2}]$ and $[\mathbf{Y 3}$, which form the scope of the research work that is central for the present thesis.

\subsection{Dual ground state solutions for the critical nonlinear Helmholtz equation.}

The first presented article [Y1] is a joint work with Gilles Evéquoz and will be concerned with the Sobolev-critical case for the nonlinear Helmholtz equation (1.1). Namely we will consider the problem

$$
-\Delta u-u=Q(x)|u|^{2^{*}-2} u, \quad \text { in } \mathbb{R}^{N}
$$

for $N \geq 3, Q \in L^{\infty}\left(\mathbb{R}^{N}\right) \backslash\{0\}$ non-negative and $2^{*}:=\frac{2 N}{N-2}$.

As already pointed out in Lemma 1.7, the (bounded) Birman-Schwinger operator $\mathbf{K}_{Q}:=$ $\mathbf{K}_{Q, 2^{*}}: L^{2^{+}}\left(\mathbb{R}^{N}\right) \rightarrow L^{2^{*}}\left(\mathbb{R}^{N}\right)$, where $2^{+}:=\left(2^{*}\right)^{\prime}=\frac{2 N}{N+2}$, given by

$$
\mathbf{K}_{Q}(f)=Q^{\frac{1}{p}} \mathbf{R}\left(Q^{\frac{1}{p}} f\right)
$$

fails even to be locally compact in the Sobolev-critical case, which is due to the lack of compactness in the underlying Sobolev-embedding. We will demonstrate, how the Palais-Smale condition for the dual functional $\left.J_{Q, 2^{*}}=: J_{Q}: L^{2^{+}} \mathbb{R}^{N}\right) \rightarrow L^{2^{*}}\left(\mathbb{R}^{N}\right)$ given by

$$
J_{Q}(u)=\frac{1}{2^{+}}\|u\|_{L^{2^{+}}\left(\mathbb{R}^{N}\right)}^{2^{+}}-\frac{1}{2} \int_{\mathbb{R}^{N}} u \mathbf{K}_{Q}(u) d x
$$

can be recovered for certain energy levels. To this purpose, we compare the Mountain-Pass level $L_{Q}$ of $J_{Q}$ with the least energy level $L_{Q}^{*}$ among all possible limit problems

$$
-\Delta u=Q\left(x_{0}\right)|u|^{p-2} u, \quad \text { in } \mathbb{R}^{N}
$$

for some $x_{0} \in \mathbb{R}^{N}$, similar to the classical case of a bounded domain in the celebrated work of Brézis and Nirenberg [10]. We shall see, that

$$
L_{Q}^{*}=\frac{S^{\frac{N}{2}}}{N\|Q\|_{\infty^{\frac{N-2}{2}}}}
$$

where $S$ denotes the optimal constant in the Sobolev inequality (see [46, §8]). Our strategy in order to obtain critical points of $J_{Q}$ can be summarized by the following two steps: 
(I) Show that at every level $0<\beta<L_{Q}^{*}$, the Palais-Smale condition is satisfied, and

(II) establish the strict inequality $L_{Q}<L_{Q}^{*}$.

The comparison of (1.31) and (1.32) takes place on the level of corresponding fundamental solutions (and resolvent operators) since we do not have a direct functional for (1.31) in hand. The following lemma regarding the difference $\Psi-\Lambda: \mathbb{R}^{N} \backslash\{0\} \rightarrow \mathbb{R}$, where $\Psi, \Lambda: \mathbb{R}^{N} \backslash\{0\} \rightarrow \mathbb{R}$ are the fundamental solutions associated with the Helmholtz and Laplace's equation is crucial to obtain both steps (I) and (II).

Lemma 1.11. Let $r>0$ be given such that $r<y_{\frac{N-4}{2}}$ if $N \geq 4$, where $y_{\frac{N-4}{2}}$ is the first positive zero of $Y_{\frac{N-4}{2}}$ and $r<\pi$ if $N=3$.

(i) There exist $\kappa_{1}, \kappa_{2}>0$ only depending on $r$ and $N$, such that for all $x \in B_{r}(0)$,

$$
\begin{cases}\kappa_{1}|x|^{4-N} \leq \Psi(x)-\Lambda(x) \leq \kappa_{2}|x|^{4-N}, & \text { if } N \geq 5, \\ \kappa_{1}|\ln | x|| \leq \Psi(x)-\Lambda(x) \leq \kappa_{2}|\ln | x||, & \text { if } N=4, \\ -\kappa_{1}|x| \geq \Psi(x)-\Lambda(x) \geq-\kappa_{2}|x|, \quad \text { if } N=3 .\end{cases}
$$

(ii) For every multiindex $\alpha \in \mathbb{N}_{0}^{N}$ with $|\alpha| \geq 1$, there exists $\kappa_{3}>0$ only depending on $|\alpha|$, $r$ and $N$, such that

$$
\left|\partial^{\alpha}(\Psi(x)-\Lambda(x))\right| \leq \kappa_{3}|x|^{4-N-|\alpha|}, \quad \text { for all } x \in B_{r}(0) .
$$

The proof of Lemma 1.11 relies heavily on a detailed study of Bessel-functions for small arguments. A first consequence of Lemma 1.11 is a new compactness result for the difference $\mathbf{R}-\mathbf{R}_{0}: L^{2^{+}}\left(\mathbb{R}^{N}\right) \rightarrow L_{l o c}^{2^{*}}\left(\mathbb{R}^{N}\right)$, where $\mathbf{R}_{0}: f \mapsto \Lambda * f$ is the resolvent operator associated with Laplace's equation.

Proposition 1.12.

(i) The difference $\mathbf{R}-\mathbf{R}_{0}$ is a continuous linear mapping from $L^{2^{+}}\left(\mathbb{R}^{N}\right)$ into $W^{2,2^{*}}\left(\mathbb{R}^{N}\right)$.

(ii) For all $r>0$, the operator $\mathbb{1}_{B_{r}}\left(\mathbf{R}-\mathbf{R}_{0}\right): L^{2^{+}}\left(\mathbb{R}^{N}\right) \rightarrow L^{2^{*}}\left(\mathbb{R}^{N}\right)$ is compact.

Together with the result, that $\mathbf{R}: L^{2^{+}}\left(\mathbb{R}^{N}\right) \rightarrow L^{t}\left(\mathbb{R}^{N}\right)$ remains locally compact for subcritical $t \in\left[1,2^{*}\right)$, we are able to finish step (I). By proving that the nonvanishing Theorem 1.9 continues to hold for $p=2^{*}$, we are able to additionally include periodic $Q$ into our considerations. Regarding step (I) thus we have the following result.

Proposition 1.13. Let $Q \in L^{\infty}\left(\mathbb{R}^{N}\right) \backslash\{0\}$ have the form $Q=Q_{p e r}+Q_{0}$, for some $Q_{p e r}, Q_{0} \geq 0$ such that $Q_{\text {per }}$ is $\mathbb{Z}^{N}$-periodic and $Q_{0}(x) \rightarrow 0$ as $|x| \rightarrow \infty$.

If $\left(v_{n}\right)_{n} \subset L^{2^{+}}\left(\mathbb{R}^{N}\right)$ is a Palais-Smale sequence at level $\beta$ for $J_{Q}$ such that $\beta=L_{Q}<L_{Q}^{*}$, then there exists $w \in L^{2^{+}}\left(\mathbb{R}^{N}\right), w \neq 0$, such that $J_{Q}^{\prime}(w)=0$ and $J_{Q}(w)=L_{Q}$. 
In order to estimate the Mountain-Pass Level $L_{Q}$, we note that in our situation we have the alternative description

$$
L_{Q}=\inf \left\{\frac{1}{N}\left(\frac{\int_{\mathbb{R}^{N}}|v|^{2^{+}} d x}{\int_{\mathbb{R}^{N}} v \mathbf{K}_{Q} v d x}\right)^{\frac{N}{2}}: v \in L^{2^{+}}\left(\mathbb{R}^{N}\right) \text { with } \int_{\mathbb{R}^{N}} v \mathbf{K}_{Q} v d x>0\right\}
$$

from which we are able to deduce that $L_{Q}$ coincides with the least-energy level, i.e.

$$
L_{Q}=\inf \left\{J_{Q}(v): v \in L^{2^{+}}\left(\mathbb{R}^{N}\right) \text { with } J_{Q}^{\prime}(v)=0\right\} .
$$

Thus, the solutions we obtain are (dual) ground state solutions, namely solutions $u \in L^{2^{*}}\left(\mathbb{R}^{N}\right)$ of the form $u=\mathbf{R}\left(Q^{\frac{1}{2^{+}}} v\right)$, where $v \in L^{2^{+}}\left(\mathbb{R}^{N}\right)$ is a critical point of $J_{Q}$ with $J_{Q}(v)=L_{Q}$ and thus has least energy among all (nontrivial) critical points. Note that by Remark 1.5, these are indeed strong solutions of (1.31). To verify step (II), we shall estimate the quantity

$$
\frac{\left\|v_{\varepsilon, \alpha}\right\|_{2^{+}}^{2^{+}}}{\int_{\mathbb{R}^{N}} v_{\varepsilon, \alpha} \mathbf{K}_{Q} v_{\varepsilon, \alpha} d x}
$$

where $v_{\varepsilon, \alpha} \in L^{2^{+}}\left(B_{2 \alpha}(0)\right)$ for some $\alpha>0$ is a smoothly cut version of the optimizer $v_{\varepsilon} \in$ $L^{2^{+}}\left(\mathbb{R}^{N}\right)$ of the Hardy-Littlewood-Sobolev inequality, thus

$$
\int_{\mathbb{R}^{N}} v_{\varepsilon} \mathbf{R}_{0} v_{\varepsilon} d x=S^{-1}\left\|v_{\varepsilon}\right\|_{2^{+}}^{2^{+}}
$$

given (up to translation and scalar multiples) by

$$
v_{\varepsilon}(x)=(N(N-2) \varepsilon)^{\frac{N+2}{4}}\left(\frac{1}{\varepsilon+|x|^{2}}\right)^{\frac{N+2}{2}}, \quad \varepsilon>0 .
$$

In this step again the difference estimated in Lemma 1.11 plays a key role and the sign-change between dimensions $N \geq 4$ and $N=3$ has an important impact. Whereas we are able to deduce $L_{Q}<L_{Q}^{*}$ for $N \geq 4$, we indeed obtain equality for $N=3$. Additionally we deduce that the Mountain-Pass level in this case is not achieved, which will result in a nonexistence result for our approach. Our main result is as follows.

Theorem 1.14. Let $N \geq 3$ and consider $Q \in L^{\infty}\left(\mathbb{R}^{N}\right) \backslash\{0\}$ such that $Q \geq 0$ a.e. in $\mathbb{R}^{N}$.

(i) If $N \geq 4$ and $Q$ satisfies

(Q1) $Q=Q_{0}+Q_{\mathrm{per}}$, where $Q_{0}, Q_{\mathrm{per}} \geq 0$ are such that $Q_{\mathrm{per}}$ is periodic and $Q_{0} \rightarrow 0$ as $|x| \rightarrow \infty$.

(Q2) There exists $x_{0} \in \mathbb{R}^{N}$ with $Q\left(x_{0}\right)=\max _{\mathbb{R}^{N}} Q$ and as $\left|x-x_{0}\right| \rightarrow 0$ we have

$$
Q(x)-Q\left(x_{0}\right)= \begin{cases}o\left(\left|x-x_{0}\right|^{2}\right), & \text { if } N \geq 5 \\ O\left(\left|x-x_{0}\right|^{2}\right), & \text { if } N=4 .\end{cases}
$$

Then, the problem 1.31 has a dual ground state solution. 
(ii) If $N=3$, then no dual ground state exists for (1.31).

The additional condition (Q2) is sufficient in order to prove $L_{Q}<L_{Q}^{*}$ for $N \geq 4$. Since we are considering non-constant $Q$, we need a condition that controls at which rate $Q$ attains its maximal value $\|Q\|_{\infty}$. This condition appears in several papers concerned with critical problems (see [25]) and was also used by Chabrowski and Szulkin in [14] in the context of critical Schrödinger equations. Egnell [24] provides examples of critical problems, where this condition is also necessary.

\subsection{Fourier-extension estimates for symmetric functions and applications to} nonlinear Helmholtz equations. The second contribution [Y2], which is a joint work with Tobias Weth, is concerned with the existence of (non-radial) real-valued solutions of

$$
-\Delta u-u=Q(x)|u|^{p-2} u, \quad \text { in } \mathbb{R}^{N}
$$

with $Q \in L^{\infty}\left(\mathbb{R}^{N}\right) \backslash\{0\}$ allowing for values $2<p \leq 2_{*}$. To this purpose, we study implications of improved Fourier-extension estimates with regard to boundedness properties of the Helmholtz resolvent operator and related nonvanishing results. Moreover, we provide an improved Fourierextension estimate, that explicitly allows to treat values $q<2 *$ and study its consequences for the dual variational framework regarding (1.34).

Motivated by the fact that Fourier-extension results like Theorem 1.3 can be improved both, by considering additional symmetries and considering weighted norms, we introduce the notion of an admissible extension triple. That is a triple $(G, q, Q)$, with a closed subgroup $G \subset O(N)$, $q \geq 1$ and $Q: \mathbb{R}^{N} \rightarrow \mathbb{C}$ measurable, such that the (weighted) Fourier-extension estimate

$$
\left\|Q \check{F}_{\sigma}\right\|_{L^{p}\left(\mathbb{R}^{N}\right)} \leq C\|F\|_{L^{2}\left(\mathbb{S}^{N-1}\right)} \quad \text { for every } G \text {-invariant function } F \in L^{2}\left(\mathbb{S}^{N-1}\right)
$$

holds for a constant $C>0$ independent of $F$. Here, a function $F \in L^{2}\left(\mathbb{S}^{N-1}\right)$ is called $G$-invariant, if $F(A \cdot)=F$ holds for every $A \in G$. Thus, from Theorem 1.3 we deduce that $(\{\mathrm{id}\}, q, 1)$ is an admissible extension triple for all $q \geq 2_{*}$ and its radial version yields that $(O(N), q, 1)$ is an admissible extension triple for any $q>\frac{2 N}{N-1}$. Let us mention, that for $Q \in L^{s}\left(\mathbb{R}^{N}\right)$ for $s \in[1, \infty)$ the triple $(\{\mathrm{id}\}, q, Q)$ is an admissible extension triple for all $q \geq \max \{1, L(s, N)\}$, where $L(s, N):=\frac{2(N+1)}{2(N+1) / s+(N-1)} \rightarrow 2_{*}$ from below as $s \rightarrow \infty$, which is a consequence of Hölder's inequality applied to the LHS of 1.35. We are interested in admissible extension triples with $Q \in L^{\infty}\left(\mathbb{R}^{N}\right)$ for which we do not achieve an improvement by decay assumptions on $Q$, but from the underlying symmetry assumption. To this purpose, we concentrate on $G=G_{k}:=O(N-k) \times O(k)$ with $k \in\{1, \ldots, N-1\}$ and weight functions $Q=Q_{\alpha}=\mathbb{1}_{L_{\alpha}}$, where

$$
L_{\alpha}:=\left\{x=\left(x^{(N-k)}, x^{(k)}\right) \in \mathbb{R}^{N-k} \times \mathbb{R}^{k}:\left|x^{(N-k)}\right| \leq a\left|x^{(k)}\right|^{-\alpha}\right\}
$$

for $a, \alpha>0$. Since $\left|L_{\alpha}\right|=\infty$ for any $\alpha>0$, we have $Q_{\alpha} \in L^{\infty}\left(\mathbb{R}^{N}\right) \backslash L^{s}\left(\mathbb{R}^{N}\right)$ for all $s \in[1, \infty)$. We can state the following result. 
Theorem 1.15. Let $N \geq 3, k \in\{1, \ldots, N-1\}$, let $\alpha>0$, and let $Q_{\alpha}=\mathbb{1}_{L_{\alpha}}$ be given as above. Moreover, suppose that

$$
\alpha>\frac{1}{N-1} \quad \text { if } k=1, \quad \alpha<N-1 \quad \text { if } k=N-1,
$$

and let

$$
\lambda_{N, k, \alpha}:= \begin{cases}\frac{2(N-1)-\frac{2}{\alpha}}{N-2}, & \text { if } k=1 ; \\ \max \left\{\frac{2(N-k)-\frac{2 k}{\alpha}}{N-k-1}, \frac{2 k-2 \alpha(N-k)}{k-1}\right\} & \text { if } 2 \leq k \leq N-2 ; \\ \frac{2(N-1)-2 \alpha}{N-2}, & \text { if } k=N-1 .\end{cases}
$$

Then $\left(G_{k}, q, Q_{\alpha}\right)$ is an admissible extension triple for every $q>\lambda_{N, k, \alpha}$.

We shall in fact prove a generalization by using

$$
L_{\alpha, \beta}:=\left\{x=\left(x^{(N-k)}, x^{(k)}\right) \in \mathbb{R}^{N-k} \times \mathbb{R}^{k}:\left|x^{(N-k)}\right| \leq a \max \left\{\left|x^{(k)}\right|^{-\alpha},\left|x^{(k)}\right|^{-\beta}\right\}\right\}
$$

for $\alpha \geq \beta>0$. Note, that Theorem 1.15 also allows values $q<2 *$ as indicated in the following figure and which can be easily seen by an examination of $\lambda_{N, k, \alpha}$.

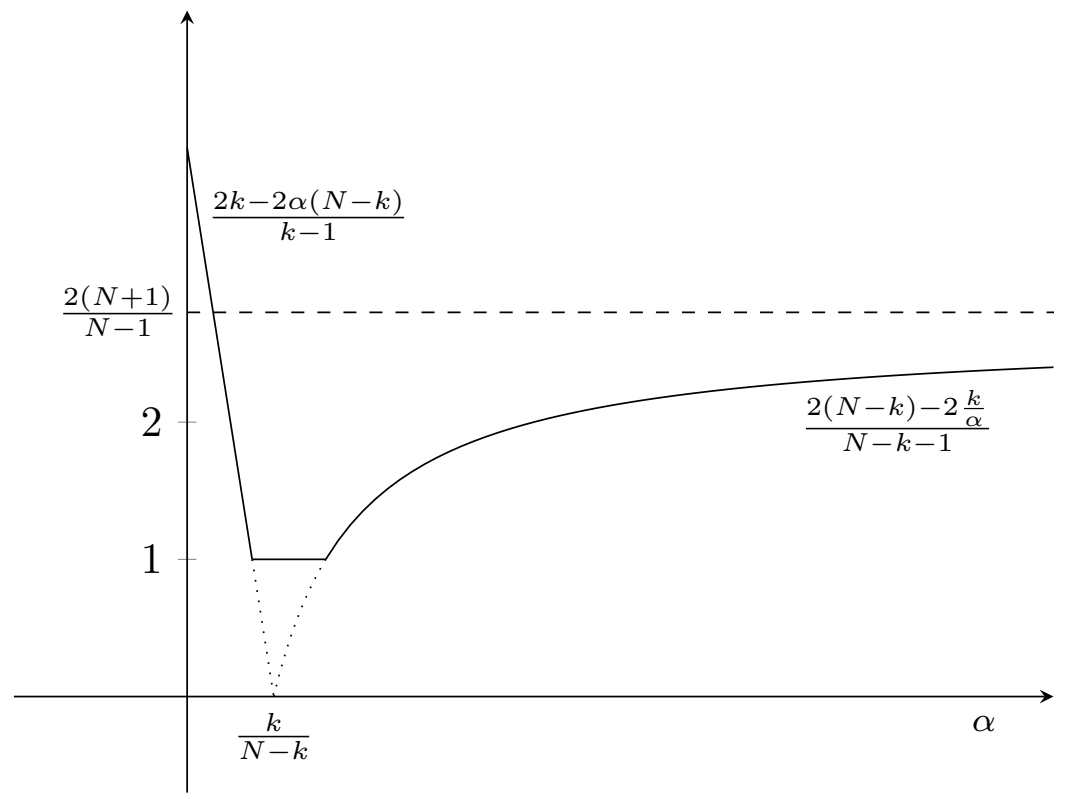

Figure 1. Admissible values of $q$ for $N=6$ and $k=2$ depending on $\alpha$

The proof of Theorem 1.15 is obtained in two steps. First, using the symmetries we can rewrite $F$ as a function $h_{F}$ on $[0,1]$ via

$$
h_{F}:[0,1] \rightarrow \mathbb{R}, \quad h_{F}(r):=F\left(r \eta, \sqrt{1-r^{2}} \mu\right), \quad \text { for } \eta \in \mathbb{S}^{N-k-1}, \mu \in \mathbb{S}^{k-1} .
$$


Then $\|Q \check{F}\|_{L^{q\left(\mathbb{R}^{N}\right)}}$ reduces to an one-dimensional integral involving the quantities $d \check{\sigma}_{N-k}$ and $d \check{\sigma}_{k}$ whose asymptotics have to be estimated precisely. Here the bound (1.13) plays a key role. We then study abstract implications of admissible extension triples with regard to corresponding resolvent and nonvanishing estimates. In particular we obtain, that admissible extension triples $(G, q, Q)$, for $G$-invariant $Q \in L^{\infty}\left(\mathbb{R}^{N}\right)$ give rise to resolvent and nonvanishing results for the weighted resolvent $\mathcal{R}_{Q}: f \mapsto Q[\Phi *(Q f)]$ for values $p \in\left(\frac{2 N}{N-1} \frac{2 q}{q+2}, 2^{*}\right]$. Note here that $q<2 *$ is equivalent to $\frac{2 N}{N-1} \frac{2 q}{q+2}<2_{*}$. The full result reads as follows:

Theorem 1.16. Let $N \geq 3, G \subset O(N)$ a closed subgroup, $Q \in L^{\infty}\left(\mathbb{R}^{N}\right)$ be $G$ - invariant, and let $q \in\left[1, \frac{2(N+1)}{N-1}\right]$ be such that $(G, Q, q)$ is an admissible extension triple. Then for every $p \in\left(\frac{2 N}{N+1} \frac{2 q}{2+q}, 2^{*}\right]$ there exists a constant $C>0$ such that

$$
\left\|\mathcal{R}_{Q}(f)\right\|_{L^{p}\left(\mathbb{R}^{N}\right)} \leq C\|f\|_{L^{p^{\prime}}\left(\mathbb{R}^{N}\right)}
$$

holds for all $G$-invariant Schwartz-functions $f \in \mathcal{S}\left(\mathbb{R}^{N}\right)$.

In fact we will prove a non-selfdual generalization of this statement (see Theorem 3.12 below) based on the strategy used in [37] and [43], see also [31]. Under the same assumptions as in Theorem 1.16 we are able to establish a nonvanishing result in the spirit of Theorem 1.9 .

Theorem 1.17. Let $N \geq 3$, let $G \subset O(N)$ be a closed subgroup, let $Q \in L^{\infty}\left(\mathbb{R}^{N}\right)$ be $G-$ invariant, and let $q \in\left[1, \frac{2(N+1)}{N-1}\right]$ be such that $(G, q, Q)$ is an admissible extension triple. Moreover, let $p \in\left(\frac{2 N}{N-1} \frac{2 q}{q+2}, \frac{2 N}{N-2}\right]$. Then for every $G$-invariant bounded sequence $\left(v_{n}\right)_{n} \subset$ $L^{p^{\prime}}\left(\mathbb{R}^{N}\right)$ satisfying

$$
\left|\limsup _{n \rightarrow \infty} \int_{\mathbb{R}^{N}} v_{n} \mathcal{R}_{Q}\left(v_{n}\right) d x\right|>0,
$$

there exist - after passing to a subsequence - numbers $R, \zeta>0$ and a sequence of points $\left(x_{n}\right)_{n \in \mathbb{N}} \subset \mathbb{R}^{N}$ with

$$
\int_{B_{R\left(x_{n}\right)}}\left|Q v_{n}(x)\right|^{p^{\prime}} d x \geq \zeta, \quad \text { for all } n .
$$

Let us note, that for $G=\{\operatorname{id}\}$ and $Q \equiv 1$ this reduces to Theorem 1.9 , since $\frac{2 N}{N-1} \frac{2 q}{q+2}=2 *$ for $q=2_{*}$.

Equipped with these results, we are able to deduce our first existence result for $G$-invariant solutions of 1.34 with a $G$-invariant weight function $Q \in L^{\infty}\left(\mathbb{R}^{N}\right) \backslash\{0\}$ and a closed subgroup $G \subset O(N)$. Let us state our first existence result concerning $G$-invariant dual bound states.

Theorem 1.18. Let $N \geq 3$, let $G \subset O(N)$ be a closed subgroup, and let $Q \in L^{\infty}\left(\mathbb{R}^{N}\right)$ be a real-valued, $G$-invariant, non-negative function with $Q \not \equiv 0$ and with the property that

$$
\|Q\|_{L^{1}\left(B_{R}(x)\right)} \rightarrow 0 \quad \text { as }|x| \rightarrow \infty \text { for some } R>0 .
$$


Moreover, let $q \in\left[1, \frac{2(N+1)}{N-1}\right]$, and let $p \in\left(\max \left\{\frac{2 N}{N-1} \frac{2 q}{q+2}, 2\right\}, \frac{2 N}{N-2}\right)$ be such that $\left(G, q, Q^{\frac{1}{p}}\right)$ is an admissible extension triple. Then (1.34) admits a nontrivial $G$-invariant dual bound state solution.

Here we need to ensure, that the Mountain-Pass geometry of the underlying energy functional is preserved by assuming $p>2$. By Theorem 1.3 the triple $\left(G, q, Q^{\prime}\right)$ is an admissible extension triple for $q=2_{*}$ and any closed subgroup $G \subset O(N)$ and every $Q^{\prime} \in L^{\infty}\left(\mathbb{R}^{N}\right)$. Since $\frac{2 N}{N-1} \frac{2 q}{q+2}=$ $2_{*}$ for $q=2_{*}$, we have the following consequence of Theorem 1.18 ,

Corollary 1.19. Let $N \geq 3, G \subset O(N)$ be a closed subgroup and let $Q \in L^{\infty}\left(\mathbb{R}^{N}\right) \backslash\{0\}$ be a non-negative and $G$-invariant function that satisfies (1.38). Then (1.34) admits a nontrivial $G$-invariant dual bound state solution for every $p \in\left(2_{*}, 2^{*}\right)$.

This corollary in particular applies to the non-symmetric case $G=\{$ id $\}$ but requires the asymptotic condition $(1.38)$ to hold. In the case of special invariances with respect to the group action $G_{k}=O(N-k) \times O(k)$, we may drop assumption (1.38) to obtain a nontrivial $G_{k}$-invariant dual bound state in the noncritical range $p \in\left(2_{*}, 2^{*}\right)$.

Theorem 1.20. Let $N \geq 4$, let $k \in\{2, \ldots, N-2\}$, and let $Q \in L^{\infty}\left(\mathbb{R}^{N}\right)$ be a non-negative $G_{k}$-invariant function with $Q \not \equiv 0$. Then (3.4) admits a nontrivial $G_{k}$-invariant dual bound state solution for every $p \in\left(\frac{2(N+1)}{N-1}, \frac{2 N}{N-2}\right)$.

In this situation, the restrictions on $N$ and $k$ are necessary, since this guarantees the minimal orbit dimension of $G_{k}$ to be 1 . This is required in order to prove that the sequence $\left(x_{n}\right)_{n} \subset \mathbb{R}^{N}$ obtained after applying a nonvanishing theorem is bounded. Indeed, combining the nonvanishing result with the assumed symmetries, we find that the Palais-Smale sequence $\left(u_{n}\right)_{n} \subset L^{p}\left(\mathbb{R}^{N}\right)$ has positive $L^{p^{\prime}}$-mass on any orbit of $B_{R}\left(x_{n}\right)$ and obtain a contradiction since they are bounded.

Finally, we point out that assumption (1.38) holds in particular for functions $Q \in L^{\infty}\left(\mathbb{R}^{N}\right)$ satisfying $|Q| \leq c \mathbb{1}_{L_{\alpha}}$ for some $c, \alpha>0$. Using this fact, the following corollary can be deduced from Theorems 1.15 and 1.18 .

Corollary 1.21. Let $N \geq 3$, let $k \in\{1, \ldots, N-1\}$, and let $\alpha>0$. Moreover, let $Q \in L^{\infty}\left(\mathbb{R}^{N}\right)$ be a non-negative $G_{k}$-invariant function with $Q \not \equiv 0$ and satisfying $|Q| \leq c \mathbb{1}_{L_{\alpha}}$ for some $c>0$. Then (1.34) admits a nontrivial $G_{k}$-invariant dual bound state solution for all $p \in\left(\mu_{N, k, \alpha}, \frac{2 N}{N-2}\right)$ if one of the following holds:

(i) $k=1$ and

$$
\mu_{N, 1, \alpha}:= \begin{cases}2, & \frac{1}{N-1}<\alpha \leq \frac{N+1}{3(N-1)}, \\ \frac{4 N(\alpha(N-1)-1)}{(N-1)(2 \alpha N-3 \alpha-1)}, & \alpha>\frac{N+1}{3(N-1)} .\end{cases}
$$


(ii) $k=N-1$ and

$$
\mu_{N, N-1, \alpha}:= \begin{cases}\frac{4 N(N-1-\alpha)}{(N-1)(2 N-\alpha-3)}, & 0<\alpha \leq \frac{3(N-1)}{N+1}, \\ 2, & \frac{3(N-1)}{N+1}<\alpha<N-1 .\end{cases}
$$

(iii) $2 \leq k \leq N-2$ and

$$
\mu_{N, k, \alpha}:= \begin{cases}\frac{4 N(k-\alpha(N-k))}{(N-1)(2 k-1-\alpha(N-k))}, & \alpha \leq \frac{N+2 k-1}{(N+1)(N-k)}, \\ 2, & \frac{N+2 k-1}{(N+1)(N-k)}<\alpha \leq \frac{(N+1) k}{N-1+2(N-k)}, \\ \frac{4 N(\alpha(N-k)-k)}{(N-1)(2 \alpha(N-k)-\alpha-k)}, & \alpha>\frac{(N+1) k}{N-1+2(N-k)} .\end{cases}
$$

2.3. Dual variational methods for a nonlinear Helmholtz equation with signchanging nonlinearity. Our third contribution [Y3], which resulted from a collaboration with Rainer Mandel and Dominic Scheider, demonstrates how sign-changing weight-functions $Q \in L^{\infty}\left(\mathbb{R}^{N}\right)$ can be considered in the dual variational framework for the problem

$$
-\Delta u-k^{2} u=Q(x)|u|^{p-2} u \quad \text { on } \mathbb{R}^{N}, \quad p \in\left[2_{*}, 2^{*}\right], \quad k>0 .
$$

Besides the established case of non-negative $Q$ in [31], in [52 the applicability of the dual method for non-positive $Q$ is demonstrated. In this situation the authors introduce the transformation $v=|Q|^{\frac{1}{p^{\prime}}}|u|^{p-2} u$. Then the integral equation $u=\mathbf{R}_{k}\left(Q|u|^{p-2} u\right)$ leads to

$$
|v|^{p^{\prime}-2} v=-|Q|^{\frac{1}{p^{\prime}}} \mathbf{R}_{k}\left(|Q|^{\frac{1}{p^{\prime}}}|u|^{p-2} u\right)
$$

which again has a variational structure (see [52, §3]). Let us remark, that the right hand side has a negative sign since $Q=-|Q|$ in the case of non-positive $Q$. Thus for sign-changing weight functions a new method needs to be developed. Our idea is as follows: Write $Q=Q_{+}-Q_{-}$with $Q_{ \pm}=|Q| \mathbb{1}_{A_{ \pm}}$, where $A_{+}:=\{Q>0\}$ and $A_{-}:=\{Q<0\}$. We then introduce $Q_{\lambda}:=\lambda Q_{+}-Q_{-}$ for $\lambda>0$. Then, for the integral equation

$$
u=\mathbf{R}_{k}\left(Q_{\lambda}|u|^{p-2} u\right), \quad u \in L^{p}\left(\mathbb{R}^{N}\right)
$$

we consider the restrictions $v:=\left.u\right|_{A_{+}}$and $w:=\left.u\right|_{A_{-}}$which leads to the system

$$
\begin{aligned}
v & =\lambda \mathbb{1}_{A_{+}} \mathbf{R}_{k}\left[Q_{+}|v|^{p-2} v\right]-\mathbb{1}_{A_{+}} \mathbf{R}_{k}\left[Q_{-}|w|^{p-2} w\right], \\
w & =\lambda \mathbb{1}_{A_{-}} \mathbf{R}_{k}\left[Q_{+}|v|^{p-2} v\right]-\mathbb{1}_{A_{-}} \mathbf{R}_{k}\left[Q_{-}|w|^{p-2} w\right] .
\end{aligned}
$$

Applying the dual transformations

$$
\varphi:=\lambda Q_{+}^{1 / p^{\prime}}|v|^{p-2} v \in L^{p^{\prime}}\left(A_{+}\right), \quad \psi:=Q_{-}^{1 / p^{\prime}}|w|^{p-2} w \in L^{p^{\prime}}\left(A_{-}\right)
$$


the above system leads to a "dual" system

$$
\begin{aligned}
\lambda^{1-p^{\prime}}|\varphi|^{p^{\prime}-2} \varphi & =Q_{+}^{1 / p} \mathbf{R}_{k}\left(|Q|^{1 / p}(\varphi-\psi)\right)=\mathbb{1}_{A_{+}} \mathcal{K}_{k}(\varphi-\psi), \\
|\psi|^{p^{\prime}-2} \psi & =Q_{-}^{1 / p} \mathbf{R}_{k}\left(|Q|^{1 / p}(\varphi-\psi)\right)=\mathbb{1}_{A_{-}} \mathcal{K}_{k}(\varphi-\psi)
\end{aligned}
$$

with the Birman-Schwinger operator $\mathcal{K}_{k}: L^{p^{\prime}}\left(\mathbb{R}^{N}\right) \rightarrow L^{p}\left(\mathbb{R}^{N}\right)$ given by

$$
\mathcal{K}_{k}(f)=|Q|^{1 / p} \mathbf{R}_{k}\left(|Q|^{1 / p} f\right) .
$$

This system carries for $p \in\left[2_{*}, 2^{*}\right]$ a variational structure through the dual energy-functional $J_{\lambda}: L^{p^{\prime}}\left(A_{+}\right) \times L^{p^{\prime}}\left(A_{-}\right) \rightarrow \mathbb{R}$ given by

$$
J_{\lambda}(\varphi, \psi):=\frac{\lambda^{1-p^{\prime}}}{p^{\prime}}\|\varphi\|_{p^{\prime}}^{p^{\prime}}-\frac{1}{p^{\prime}}\|\psi\|_{p^{\prime}}^{p^{\prime}}-\frac{1}{2} \int_{\mathbb{R}^{N}}(\varphi-\psi) \mathcal{K}_{k}(\varphi-\psi) d x .
$$

The functional $J_{\lambda}$ is of class $\mathcal{C}^{1}$ and nontrivial critical points of $J_{\lambda}$ correspond to nontrivial solutions of the problem

$$
-\Delta u-k^{2} u=Q_{\lambda}(x)|u|^{p-2} u \quad \text { on } \mathbb{R}^{N} .
$$

Contrary to the non sign-changing case, the functional $J_{\lambda}$ does not have a Mountain-Pass structure and the boundedness of Palais-Smale (or Cerami) sequences turns out to be a difficult problem.

We establish the existence of critical points for $J_{\lambda}$ in two steps. As a first step, we perform a saddle-point reduction with respect to the $\psi$-variable. In particular we prove, that if $\mathcal{K}_{k}$ is compact and $\int_{\mathbb{R}^{N}} \psi \mathcal{K}_{k}(\psi) d x \geq 0$ for all $\psi \in L^{p^{\prime}}\left(A_{-}\right)$, then $\psi \mapsto J_{\lambda}(\varphi, \psi)$ is concave and bounded from above. Thus we are able to verify, that for any $\varphi \in L^{p^{\prime}}\left(A_{+}\right)$there exists a unique $\psi^{*} \in L^{p^{\prime}}\left(A_{-}\right)$such that

$$
J_{\lambda}\left(\varphi, \psi^{*}\right)=\max _{\psi \in L^{p^{\prime}}\left(A_{-}\right)} J(\varphi, \psi):=\tilde{J}_{\lambda}(\varphi)
$$

This result holds for any $\lambda>0$ and the obtained maximizer is independent of $\lambda$. Thus we are led to study the reduced functional $\tilde{J}_{\lambda}$ that for $\lambda>\lambda_{0}$ has a Mountain-Pass structure. Here $\lambda_{0}:=\left(2 \beta \alpha^{-1}\right)^{p}$ with

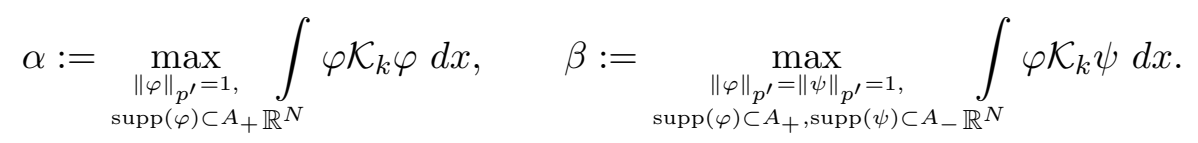

For compact $\mathcal{K}_{k}$, both values are attained and we have $\beta \geq 0$. Furthermore we deduce $\alpha>0$ if $\left|A_{+}\right|>0$, as can be seen by the same arguments as in the proof of [31, Lemma 4.2 (ii)]. Since $\tilde{J}_{\lambda}$ fulfills a monotonicity condition with respect to $\lambda$, we are allowed to apply the abstract result [39, Theorem 2.1] in order to obtain the existence of bounded Palais-Smale sequences. This theorem only provides the existence for almost all $\lambda>\lambda_{0}$ which is typical for monotonicity tricks of this kind. Let us state our main result. 
TheOREM 1.22. Let $p \in\left[\frac{2(N+1)}{N-1}, \frac{2 N}{N-2}\right)$ and $Q \in L^{\infty}\left(\mathbb{R}^{N}\right), Q^{+} \not \equiv 0$. Moreover assume that

$$
\mathcal{K}_{k}: L^{p^{\prime}}\left(\mathbb{R}^{N}\right) \rightarrow L^{p}\left(\mathbb{R}^{N}\right) \text { is compact and } \int_{\mathbb{R}^{N}} \psi \mathcal{K}_{k} \psi d x \geq 0 \text { for all } \psi \in L^{p^{\prime}}\left(A_{-}\right) .
$$

Then for almost all $\lambda>\lambda_{0}:=\left(2 \beta \alpha^{-1}\right)^{p}$ there exists a nontrivial dual bound state solution of 1.44 .

As already seen, compactness of $\mathcal{K}_{k}$ is for example given, when $Q$ vanishes at infinity. Using [15. Corollary 5.4] we see that the non-negativity assumption on the bilinearform can indeed be achieved, if $A_{-}$has a small diameter, namely if $\operatorname{diam}\left(A_{-}\right) \leq k^{-1} y_{\frac{N-2}{2}}$, where $y_{\frac{N-2}{2}}$ denotes the first positive zero of the Bessel-function $\frac{Y_{\frac{N-2}{2}}}{\text {. }}$

Corollary 1.23. Assume $p \in\left[\frac{2(N+1)}{N-1}, \frac{2 N}{N-2}\right)$ and $Q \in L^{\infty}\left(\mathbb{R}^{N}\right), Q^{+} \not \equiv 0$. Moreover assume

$$
\lim _{R \rightarrow \infty} \operatorname{esssup}_{|x| \geq R}|Q|=0 \quad \text { and } \quad \operatorname{diam}\left(A_{-}\right) \leq k^{-1} y_{\frac{N-2}{2}} .
$$

Then for almost all $\lambda>\lambda_{0}:=\left(2 \beta \alpha^{-1}\right)^{p}$ there exists a nontrivial dual bound state solution of $(1.44$. 


\section{Zusammenfassung}

Duale variationelle Methoden haben sich im Kontext nichtlinearer partieller Differentialgleichungen oftmals in Situationen bewährt, in welchen kein, oder zumindest kein einfacher direkter Zugang möglich war. Eine der ersten Arbeiten hierzu sind wohl [16], [17], wo ein „dual action principle" eingeführt wird, um die Existenz periodischer Lösungen für die Hamilton Gleichungen per Minimierung eines Integrals zu etablieren, welches „dual“ im Sinne der FenchelTransformation ist. Diese Ideen wurden in [18] erfolgreich weitergeführt. Wir möchten auch [2],[3], 11], [19, [38] sowie [9] erwähnen als z.T. moderne Beispiele, in welchen duale variationelle Methoden einen Zugang zur weiteren, tieferen Analyse des Problems zuließen.

G.Evéquoz und T.Weth haben in 31 einen dualen variationellen Rahmen für die nichtlineare Helmholtz-Gleichung

$$
-\Delta u-k^{2} u=Q(x)|u|^{p-2} u, \quad x \in \mathbb{R}^{N}
$$

mit $Q \in L^{\infty}\left(\mathbb{R}^{N}\right) \backslash\{0\}$ nicht-negativ, $k>0$ sowie $p \in\left[2_{*}, 2^{*}\right]$ für Dimensionen $N \geq 3$ aufgestellt. Hier ist $2_{*}:=\frac{2(N+1)}{N-1}$ und $2^{*}:=\frac{2 N}{N-2}$ gesetzt. Unter weiteren Bedingungen an $Q$ und $p$ erlaubt es dieser Zugang, die Existenz stehender Wellen für (1.47), welche sich aus reellwertigen Lösungen ergeben, zu beweisen. Gleichung (1.47) ist verwandt mit stationären Schrödingergleichungen der Form

$$
-\Delta u+\lambda u=f(x, u), \quad x \in \mathbb{R}^{N},
$$

welche seit den frühen 1980er Jahren Gegenstand vieler Untersuchungen sind. Dazu seien die wegweisenden Arbeiten von Berestycki und Lions [7, [8], Ding und Ni [22, Floer und Weinstein 33 sowie vielen anderen erwähnt. Während die Existenz reellwertiger Lösungen im Fall $\lambda \geq 0$ für verschiedene Klassen von Nichtlinearitäten $f$ als gut verstanden gilt (siehe z.B. [63, [69]), gibt es für den Fall $\lambda<0$ vergleichsweise wenige Resultate, welcher (1.47) mit $\lambda=-k^{2}$ sowie $f(x, u)=Q(x)|u|^{p-2} u$ entspricht. Tatsächlich erweist sich das Problem in dieser Situation als schwierig, weil die etablierten Methoden aus dem Fall $\lambda \geq 0$ nicht, oder nur begrenzt anwendbar sind. Beispielsweise hat Rellich [56] (oder auch Kato [40]) gezeigt, dass Lösungen der Helmholtz-Gleichung das Abklingverhalten

$$
u(x)=O\left(|x|^{\frac{1-N}{2}}\right), \quad \text { für }|x| \rightarrow \infty
$$

aufzeigen, demzufolge also in $L^{p}\left(\mathbb{R}^{N}\right)$ (bzw. $W^{2, p}\left(\mathbb{R}^{N}\right)$ ) für $p>\frac{2 N}{N-1}$ zu erwarten sind. Insbesondere ist also ein $L^{2}$-basierter variationeller Rahmen, wie für das natürlich mit (1.47) assoziierte Energie-Funktional, nicht ohne Weiteres möglich. Hierbei bezeichne $W^{m, p}\left(\mathbb{R}^{N}\right)$ den Sobolev-Raum schwach differenzierbarer Funktionen bis zur Ordnung $m \in \mathbb{N}$, welche in $L^{p}\left(\mathbb{R}^{N}\right)$ liegen sowie $H^{m}\left(\mathbb{R}^{N}\right):=W^{m, 2}\left(\mathbb{R}^{N}\right)$. Als weitere analytische Schwierigkeit kommt hinzu, dass nun 0 im wesentlichen Spektrum des (linearisierten) Schrödingeroperators $-\Delta-k^{2}$ liegt, welches die Anwendbarkeit etablierter funktionalanalytischer Mittel limitiert. 
In der vorliegenden Arbeit befassen wir uns mit Erweiterungen der dualen Methode aus [31. Genauer werden uns mit dem Sobolev-kritischen Fall $p=2^{*}$ befassen (Kapitel 2), die Möglichkeit zur Einbeziehung von Werten $p \leq 2_{*}$ herausarbeiten (Kapitel 3) sowie demonstrieren, wie vorzeichenwechselnde Gewichtsfunktionen in den dualen Rahmen eingebettet werden können (Kapitel 4). In all diesen Fällen werden wir insbesondere die Existenz reeller Lösungen von 1.47) unter diesen neuen Gegebenheiten beweisen.

Die vorliegende Arbeit ist wie folgt strukturiert. Wir beginnen mit einer Einleitung zur Helmholtz-Gleichung und beleuchten ihre Stellung im Kontext der Theorie von Wellenausbreitung. Dann widmen wir uns Aspekten der linearen Gleichung und führen die von uns betrachtete Fundamentallösung sowie den dazugehörigen Resolventenoperator ein und präsentieren die wichtigsten Resultate in diesem Zusammenhang, welche im Verlauf des gesamten Textes von Wichtigkeit sind. Anschließend präsentieren wir eines der ersten Resultate für einen Spezialfall der nichtlinearen Gleichung (1.47) aus [37. In dem darauf folgenden Abschnitt präsentieren wir die duale Methode aus [31], erläutern die wichtigsten Resultate in diesem Zusammenhang sowie eventuelle Limitierungen. Anschließend diskutieren wir Erweiterungen sowie verwandte Resultate. Dies ebnet uns den Weg, die dieser Dissertation zugrunde liegenden Arbeiten [Y1], [Y2], [Y3] zusammengefasst wiederzugeben und einzuordnen. Diese werden dann in den Kapiteln 2, 3 und 4 in der veröffentlichten Form präsentiert.

Die Resultate aus Kapitel 2 stammen aus einer Arbeit mit Gilles Evèquoz veröffentlicht in [Y1]. Die gemeinsame Arbeit [Y2] mit Tobias Weth wird in Kapitel 3 präsentiert. In Kapitel 4 befassen wir uns dann mit der unter [Y3] zu findenden Arbeit, welche einer Kollaboration mit Rainer Mandel und Dominic Scheider entstammt. 
3.1. Die Helmholtz-Gleichung. Reellwertige Lösungen der nichtlinearen HelmholtzGleichung stehen in engem Zusammenhang mit hochfrequenten zeit-harmonischen Lösungen der nichtlinearen Klein-Gordon Gleichung

$$
\frac{\partial^{2} \psi}{\partial t^{2}}-\Delta \psi(t, x)+m \psi(t, x)=f(x, \psi(t, x)), \quad m>0, \quad(t, x) \in \mathbb{R} \times \mathbb{R}^{N},
$$

im Falle einer superlinearen Nichtlinearität $f(x, u)=g\left(x,|\psi|^{2}\right) \psi$. Dies folgt aus dem zeitharmonischen Ansatz $\psi(t, x)=e^{\mathrm{i} k t} u(x)$ für 1.50 und führt zu

$$
-\Delta u+\left(m-\omega^{2}\right) u=f(x, u), \quad x \in \mathbb{R}^{N} .
$$

Diese Gleichung ist von der Form (1.48) mit $\lambda=m-\omega^{2}$. Wir haben demnach $\lambda<0$ für hohe Frequenzen $\omega^{2}>m$. Die Klein-Gordon Gleichung ist zentral für die mathematische Beschreibung von Wellen in (nichtlinearen) Medien, welches ein faszinierendes aber auch herausforderndes Teilgebiet der nichtlinearen Analysis ist (siehe z.B. [42 sowie 61 für weiterführendes zu diesem Thema). Demzufolge leisten reellwertige Lösungen der Helmholtz-Gleichungen einen wichtigen Beitrag zum Verständnis zeit-periodischer Lösungen und damit dem Gesamtverständnis von Wellenphänomenen.

3.2. Fundamentallösung und Resolventenoperator. Die Abklingbedingung (1.49) für Lösungen der Helmholtz-Gleichung ist vom analytischen Standpunkt her unzufrieden stellend, da, wie in [55, Kapitel 2] hervorgehoben wurde, es notwendig ist das genaue Strahlungsverhalten der Lösungen bei unendlich zu beschreiben. Im Zusammenhang mit dem linearen Problem

$$
-\Delta u-k^{2} u=f(x), \quad x \in \mathbb{R}^{N}
$$

mit einer (möglicherweise komplexwertigen) Funktion $f$ auf $\mathbb{R}^{N}$, wurden von Sommerfeld in [58] Lösungen betrachtet, welche zusätzlich die (ausgehende) Strahlungsbedingung

$$
r^{\frac{N-1}{2}}\left|\frac{\partial u}{\partial r}-\mathrm{i} k u\right| \rightarrow 0, \quad \text { für } r=|x| \rightarrow \infty
$$

erfüllen. Die dazugehörigen Lösungen sind von $f$ angeregte ausgehende Wellen. Ist $f$ hinreichend regulär (stetig und kompakt getragen), so hat (1.52), 1.53) eine eindeutige Lösung $u$, welche (formal) gegeben ist durch $u=\left(-\Delta-k^{2}\right)^{-1} f$ (siehe [20]). Wir möchten kurz vorstellen, wie dieser Resolventenoperator funktionalanalytisch beschrieben werden kann: Für jedes $\varepsilon>0$ sowie jedes $k>0$ ist der Operator $-\Delta-\left(k^{2}+\mathrm{i} \varepsilon\right): H^{2}\left(\mathbb{R}^{N}\right) \subset L^{2}\left(\mathbb{R}^{N}\right) \rightarrow L^{2}\left(\mathbb{R}^{N}\right)$ ein Isomorphismus. Für jede Schwartz-Funktion $f \in \mathcal{S}\left(\mathbb{R}^{N}\right)$ kann die Inverse dann beschrieben werden durch

$$
\mathcal{R}_{k, \varepsilon} f(x):=\left[-\Delta-\left(k^{2}+\mathrm{i} \varepsilon\right)\right]^{-1} f(x)=(2 \pi)^{\frac{N}{2}} \int_{\mathbb{R}^{N}} e^{\mathrm{i} x \cdot \xi} \frac{\widehat{f}(\xi)}{|\xi|^{2}-\left(k^{2}+\mathrm{i} \varepsilon\right)} d \xi
$$


wobei

$$
\mathcal{F}(f)(\xi)=\widehat{f}(\xi):=(2 \pi)^{-\frac{N}{2}} \int_{\mathbb{R}^{N}} e^{\mathrm{i} x \cdot \xi} f(x) d x
$$

die Fourier transformierte für $f: \mathbb{R}^{N} \rightarrow \mathbb{C}$ bezeichne. Wie in [36] beschrieben, existiert ein linearer Operator $\mathcal{R}_{k}: \mathcal{S}\left(\mathbb{R}^{N}\right) \rightarrow \mathcal{S}^{\prime}\left(\mathbb{R}^{N}\right)$ mit

$$
\left\langle\mathcal{R}_{k} f, g\right\rangle=\lim _{\varepsilon \rightarrow 0} \int_{\mathbb{R}^{N}}\left[\mathcal{R}_{k, \varepsilon} f\right](x) g(x) d x=\int_{\mathbb{R}^{N}}\left[\Phi_{k} * f\right](x) g(x) d x, \quad \text { für } f, g \in \mathcal{S}\left(\mathbb{R}^{N}\right),
$$

wobei $\Phi_{k}: \mathbb{R}^{N} \backslash\{0\} \rightarrow \mathbb{C}$ gegeben ist durch

$$
\Phi_{k}(x)=\lim _{\varepsilon \rightarrow 0}(2 \pi)^{-\frac{N}{2}} \mathcal{F}^{-1}\left(\left(|\cdot|^{2}-k^{2}-\mathrm{i} \varepsilon\right)^{-1}\right)(x)=\frac{\mathrm{i}}{4}\left(\frac{k}{2 \pi|x|}\right)^{\frac{N-2}{2}} H_{\frac{N-2}{2}}^{(1)}(k|x|) .
$$

Die Funktion $\Phi_{k}$ ist die Fundamentallösung von 1.52 assoziiert mit 1.53 und $H_{\frac{N-2}{2}}$ die Hankel-Funktion erster Art der Ordnung $\frac{N-2}{2}$ (siehe z.B. [68] für eine ausführliche Abhandlung dieser Funktionen). Abbildungseigenschaften des Resolventenoperators $\mathcal{R}_{k}$ sind essentiell für die Untersuchung des nichtlinearen Problems. Ein Schlüsselresultat in diesem Zusammenhang ist das nachfolgende Resultat von C.E. Kenig, A. Ruiz und C.D. Sogge.

Theorem 1.24. [43, Spezialfall von Theorem 2.3] Sei $p \in\left[2_{*}, 2^{*}\right]$. Dann existiert eine Konstante $C=C(N, p)>0$, sodass

$$
\left\|\mathcal{R}_{k} f\right\|_{L^{p}\left(\mathbb{R}^{N}\right)} \leq C k^{(N-2)-\frac{2 N}{p}}\|f\|_{L^{p^{\prime}\left(\mathbb{R}^{N}\right)}}
$$

für alle $f \in \mathcal{S}\left(\mathbb{R}^{N}\right)$ gilt.

Hier und im folgenden stehe $p^{\prime}:=\frac{p}{p-1}$ stets für die Hölder-konjugierte von $p \in(1, \infty)$. Demzufolge kann der Operator $\mathcal{R}_{k}$ stetig zu einem Operator $\mathcal{R}_{k}: L^{p^{\prime}}\left(\mathbb{R}^{N}\right) \rightarrow L^{p}\left(\mathbb{R}^{N}\right)$ fortgesetzt werden, sodass 1.55 für alle $f \in L^{p^{\prime}}\left(\mathbb{R}^{N}\right)$ gilt. In [37] wurden die folgenden nicht-selbstdualen Abschätzungen bezüglich des Helmholtz-Resolventenoperators $\mathcal{R}_{k}$ bewiesen.

Theorem 1.25. Seien $t \in\left[1, \frac{2 N}{N+1}\right)$ sowie $q \in\left(\frac{2 N}{N-1}, \infty\right]$ derart, dass $\frac{2}{N+1} \leq \frac{1}{t}-\frac{1}{q} \leq \frac{2}{N}$ für $N \geq 3$ gilt. Dann existiert eine Konstante $C=C(N, t, q)$, sodass

$$
\left\|\mathcal{R}_{k} f\right\|_{L^{q}\left(\mathbb{R}^{N}\right)} \leq C k^{N(1 / t-1 / q-2 / N)}\|f\|_{L^{t}\left(\mathbb{R}^{N}\right)}
$$

für alle $f \in \mathcal{S}\left(\mathbb{R}^{N}\right)$ gilt.

Offenbar folgt Theorem 1.24 aus Theorem 1.25 mit $q=p$ und $t=p^{\prime}$. Der angegebene Bereich für $t, q$ in Theorem 1.25 ist optimal, was aus einem Gegenbeispiel von Börjeson [12, S.231-233] folgt. In Bezug auf die Beweise von Theorem 1.24 und 1.25 sei erwähnt, dass die Beschränkung hinsichtlich der oberen Grenze $1 / t-1 / q \leq 2 / N$ (bzw. $p \leq 2^{*}$ im selbstdualen Fall) aus einer zugrunde liegenden Hardy-Littlewood-Sobolev Ungleichung resultiert (siehe z.B. [46, Theorem 4.3]), weswegen wir diesen Fall im folgenden als den Sobolev-kritischen Fall bezeichnen möchten. Die untere Limitierung hängt mit der Stein-Tomas Ungleichung zusammen, 
weswegen wir diesen Fall stets als den Stein-Tomas kritischen Fall bezeichnen möchten. Die Stein-Tomas Ungleichung untersucht Abbildungseigenschaften von Fourier-Fortsetzungs- bzw. Fourier-Restriktionsoperatoren. Für $F \in L^{2}\left(\mathbb{S}^{N-1}\right)$, wobei $\mathbb{S}^{N-1}:=\left\{x \in \mathbb{R}^{N}:|x|=1\right\}$ im folgenden stets die Einheitssphäre in $\mathbb{R}^{N}$ bezeichne, ist die (inverse) Fourier-Fortsetzung gegeben als

$$
\check{F}_{\sigma}(x):=(2 \pi)^{-\frac{N}{2}} \int_{\mathbb{S}^{N-1}} e^{\mathrm{i} x \cdot \omega} F(\omega) d \sigma(\omega),
$$

wobei $d \sigma$ das induzierte Lebesguemaß auf $\mathbb{S}^{N-1}$ sei. Wir formulieren nun beide (äquivalenten) Versionen der Stein-Tomas Ungleichung (siehe z.B. [59, §8 Corollary 5.4]).

THEOREM 1.26. (Stein-Tomas Fortsetzungsungleichung) Sei $F \in L^{2}\left(\mathbb{S}^{N-1}\right)$. Dann ist $\check{F}_{\sigma} \in$ $L^{q}\left(\mathbb{R}^{N}\right)$ für alle $q \geq 2$ * und wir haben

$$
\left\|\check{F}_{\sigma}\right\|_{L^{q}\left(\mathbb{R}^{N}\right)} \leq C\|F\|_{L^{2}\left(\mathbb{S}^{N-1}\right)}
$$

mit einer von $F$ unabhängigen Konstanten $C>0$.

THEOREM 1.27. (Stein-Tomas Restriktionsungleichung) Sei $1 \leq q \leq\left(2_{*}\right)^{\prime}=\frac{2(N+1)}{N+3}$ und $f \in$ $L^{q}\left(\mathbb{R}^{N}\right)$. Dann ist $\left.\widehat{f}\right|_{\mathbb{S}^{N-1}} \in L^{2}\left(\mathbb{S}^{N-1}\right)$ und es existiert eine Konstante $C>0$ unabhängig von $f$ mit

$$
\int_{\mathbb{S}^{N-1}}|\widehat{f}(\omega)|^{2} d \sigma(\omega) \leq C\|f\|_{L^{q}\left(\mathbb{R}^{N}\right)} .
$$

Mithilfe des Knapp-Beispiels (siehe z.B. [66, Kapitel 4]), bei welchem (1.57) mit der Charakteristischen Funktion einer kleinen Kugelkappe getestet wird, sieht man, dass der in Theorem 1.26 angegebenen Bereiche für $q$ optimal ist. Für radiale Funktionen, also konstante Funktionen $F \in L^{2}\left(\mathbb{S}^{N-1}\right)$ gilt Theorem 1.26 bereits für alle $q>\frac{2 N}{N-1}$ (siehe [59, §8 Proposition 5.1]). Im Gegensatz zu den Beweisen der Theoreme 1.26 und 1.27 ist der Beweis im radialen Fall vergleichsweise einfach. Dieser folgt im wesentlichen aus der Schranke

$$
d \check{\sigma}_{N}(x) \leq C(1+|x|)^{\frac{1-N}{2}}, \quad x \in \mathbb{R}^{N},
$$

wobei $d \check{\sigma}_{N}(x):=\check{F}_{\sigma}$ für $F \equiv 1$ gesetzt sei. Stein-Tomas Resultate haben zahlreichen Anwendungen in einem großen Spektrum der Mathematik, siehe hier z.B. die Übersichtsarbeiten 66, [34]. Varianten der Stein-Tomas Ungleichung spielen eine herausragenden Rolle im Zusammenhang mit Strichartz-Abschätzungen für die Wellen- sowie Schrödingergleichung (siehe [62], [41, [13]).

3.3. Komplexe Lösungen der nichtlinearen Gleichung. Eines der ersten Resultate für das nichtlineare Problem (1.47) ist von Gutiérrez [37]. Sie etabliert die Existenz (kleiner) 
Lösungen für $N=3,4, p=4$ sowie $Q= \pm 1$, welche zusätzlich die Integralversion

$$
\sup _{R>1} \frac{1}{R} \int_{B_{R}(0)}|u|^{2} d x<\infty
$$

der Abklingbedingung (1.49) erfüllen. Signifikant wichtig ist bei ihrem Ansatz folgende Beobachtung: Für $N \geq 3$ sowie $p \in\left[2_{*}, 2^{*}\right]$ ist eine (komplexwertige) Funktion $u \in W^{2, p}\left(\mathbb{R}^{N}\right)$, welche zusätzlich die Sommerfeld'sche Strahlungsbedingung 1.53 erfüllt genau dann Lösung von (1.47), falls sie ebenfalls eine Lösung der Integralgleichung

$$
u=\mathcal{R}_{k}\left(Q|u|^{p-2} u\right)+\varphi_{g}
$$

für ein $\varphi_{g} \in \mathcal{H}$ ist. Hierbei ist

$$
\mathcal{H}:=\left\{\varphi_{g}: \mathbb{R}^{N} \rightarrow \mathbb{C}: \varphi_{g}(x)=\int_{\mathbb{S}^{N-1}} e^{\mathrm{i} k x \cdot \xi} g(\xi) d \sigma(\xi) \text { für ein } g \in L^{2}\left(\mathbb{S}^{N-1}\right)\right\}
$$

der Raum sogenannter Herglotz-Wellen. Nach Theorem 1.26 ist $\varphi_{g}=(2 \pi)^{\frac{N}{2}} \check{g}_{\sigma} \in L^{q}\left(\mathbb{R}^{N}\right)$ für alle $q \geq 2_{*}$. Jedes $\varphi_{g} \in \mathcal{H}$ ist eine Lösung der linearen homogenen Helmholtz-Gleichung [37, Abschnitt 2]. Die Nichtlinearität in [37] hat die explizite Form $f(x, u)=Q|u|^{2} u$ und definiert demzufolge einen Substitutionsoperator $N_{f}: L^{p}\left(\mathbb{R}^{N}\right) \rightarrow L^{p^{\prime}}\left(\mathbb{R}^{N}\right)$ für $p=4$. Damit erhält man aus (1.61) dann das Fixpunkt-Problem

$$
u=\mathcal{R}_{k} N_{f}(u)+\varphi_{g}, \quad \text { für ein } \varphi_{g} \in \mathcal{H},
$$

welches dann mit nicht-selbstdualen Resolventenabschätzungen sowie Kontraktionsmethoden gelöst wurde.

\subsection{Dualer variationeller Rahmen für die nichtlineare Helmholtz-Gleichung.} Evèquoz und Weth [31] untersuchen die komplementäre Klasse reellwertiger Lösungen von 1.47). Für $Q \in L^{\infty}\left(\mathbb{R}^{N}\right) \backslash\{0\}$ nicht-negativ sowie $p \in\left[2_{*}, 2^{*}\right]$ stellen sie fest, dass die gesuchten Lösungen aus der Integralgleichung

$$
u=\mathbf{R}_{k}\left(Q|u|^{p-2} u\right), \quad u \in L^{p}\left(\mathbb{R}^{N}\right)
$$

gewonnen werden können. Hier sei $\mathbf{R}_{k}(f)$ der Realteil von $\mathcal{R}_{k}$ gegeben durch $\mathbf{R}_{k}(f)=\Psi_{k} * f$ mit

$$
\Psi_{k}(x)=\operatorname{Re}\left(\Phi_{k}\right)(x)=-\frac{1}{4}\left(\frac{k}{2 \pi|x|}\right)^{\frac{N-2}{2}} Y_{\frac{N-2}{2}}(k|x|), \quad x \in \mathbb{R}^{N} \backslash\{0\} .
$$

Hierbei ist $Y_{\frac{N-2}{2}}$ die Bessel-Funktion zweiter Art der Ordnung $\frac{N-2}{2}$ (siehe z.B. 68] für eine Übersicht zu Bessel-Funktionen). Ein erste entscheidende Beobachtung in diesem Zusammenhang ist, dass Lösungen von 1.63 ebenfalls 1.61) erfüllen mit $g=g_{u}$ und der nichtlinearen 
Beziehung

$$
g(\xi)=g_{u}(\xi)=-\frac{\mathrm{i}}{4}\left(\frac{k^{2}}{2 \pi}\right)^{\frac{N-2}{2}} \mathcal{F}\left(Q|u|^{p-2} u\right)(k \xi), \quad \xi \in \mathbb{S}^{N-1} .
$$

Die triviale Lösung von (1.63) ist nach Theorem 1.24 isoliert, weswegen Kontraktionsmethoden hier keine nicht-trivialen Lösungen liefern werden. Stattdessen ist die Idee, die Integralgleichung (1.63) zu einer neuen Gleichung zu transformieren, welche eine variationelle Struktur besitzt. Hierzu setzen wir der Einfachheit halber $k=1$. Allgemeine $k>0$ können dann durch geeignete Skalierung zurückgewonnen werden. Wir untersuchen demnach das Problem

$$
-\Delta u-u=Q(x)|u|^{p-2} u, \quad x \in \mathbb{R}^{N}
$$

mit der dazugehörigen Integralgleichung

$$
u=\mathbf{R}\left(Q|u|^{p-2} u\right), \quad u \in L^{p}\left(\mathbb{R}^{N}\right)
$$

mit $\mathbf{R}:=\mathbf{R}_{1}$.

Bemerkung 1.28. Nach [31, Lemma 4.3] ist für $p \in\left[2_{*}, 2^{*}\right]$ jede Lösung $u \in L^{p}\left(\mathbb{R}^{N}\right)$ von $u=\mathbf{R}\left(Q|u|^{p-2} u\right)$ für $Q \in L^{\infty}\left(\mathbb{R}^{N}\right)$ eine starke Lösung $u \in W^{2, q}\left(\mathbb{R}^{N}\right) \cap \mathcal{C}^{1, \gamma}\left(\mathbb{R}^{N}\right)$ für alle $q \in[p, \infty)$ sowie $\gamma \in(0,1)$ von (1.65). Mithilfe der selben Argumente wie in [51, S.13] kann dies verbessert werden zu $u \in W^{2, p}\left(\mathbb{R}^{N}\right) \cap \mathcal{C}^{1, \gamma}\left(\mathbb{R}^{N}\right)$ für alle $q \in\left(\frac{2 N}{N-1}, \infty\right)$ und $\gamma \in(0,1)$. Jede Lösung von (1.66) heißt duale gebundene Zustandslösung.

Zur Transformation setzen wir $v:=Q^{\frac{1}{p^{\prime}}}|u|^{p-2} u \in L^{p^{\prime}}\left(\mathbb{R}^{N}\right)$ für ein $u \in L^{p}\left(\mathbb{R}^{N}\right)$ und erhalten dann aus 1.66) die neue Gleichung

$$
|v|^{p^{\prime}-2} v=Q^{\frac{1}{p}} \mathbf{R}\left(Q^{\frac{1}{p}} v\right)=: \mathbf{K}_{Q, p}(v),
$$

welche nun in der Tat eine variationelle Struktur trägt. Lösungen dieser Gleichung entsprechen kritischen Punkten des (dualen) Energie-Funktionals $J_{Q, p}: L^{p^{\prime}}\left(\mathbb{R}^{N}\right) \rightarrow \mathbb{R}$ gegeben durch

$$
J_{Q, p}(v)=\frac{1}{p^{\prime}}\|v\|_{L^{p^{\prime}\left(\mathbb{R}^{N}\right)}}^{p^{\prime}}-\frac{1}{2} \int_{\mathbb{R}^{N}} v \mathbf{K}_{Q, p}(v) d x .
$$

Dieses Funktional ist von der Klasse $\mathcal{C}^{1}$ mit

$$
J_{Q, p}^{\prime}(v) w=\int_{\mathbb{R}^{N}}\left(|v|^{p^{\prime}-2} v-\mathbf{K}_{Q, p}(v)\right) w d x, \quad \text { für alle } v, w \in L^{p^{\prime}}\left(\mathbb{R}^{N}\right)
$$

und besitzt eine Mountain-Pass Geometrie wie im folgenden Lemma beschrieben:

Lemma 1.29. [31, Lemma 4.2] Sei $p \in\left[2_{*}, 2^{*}\right]$ und sei $Q \in L^{\infty}\left(\mathbb{R}^{N}\right) \backslash\{0\}$ mit $Q \geq 0$ fast überall auf $\mathbb{R}^{N}$. 
(i) Es existiert $\delta>0$ und $\rho \in(0,1)$ sodass $J_{Q, p}(v) \geq \delta>0$ für alle $v \in L^{p^{\prime}}\left(\mathbb{R}^{N}\right)$ mit $\|v\|_{L^{p^{\prime}\left(\mathbb{R}^{N}\right)}}=\rho$ gilt.

(ii) Es gibt ein $v_{0} \in L^{p^{\prime}}\left(\mathbb{R}^{N}\right)$ mit $\left\|v_{0}\right\|_{L^{p^{\prime}\left(\mathbb{R}^{N}\right)}}>1$ und $J_{Q, p}\left(v_{0}\right)<0$.

(iii) Jede Palais-Smale Folge für $J_{Q, p}$ ist in $L^{p^{\prime}}\left(\mathbb{R}^{N}\right)$ beschränkt.

Hierbei heiße eine Folge $\left(v_{n}\right)_{n} \subset L^{p^{\prime}}\left(\mathbb{R}^{N}\right)$ eine Palais-Smale-Folge ((PS)-Folge) zu $J_{Q, p}$, falls $\left(J_{Q, p}\left(v_{n}\right)\right)_{n} \subset \mathbb{R}$ beschränkt ist und $\left\|J_{Q, p}^{\prime}\left(v_{n}\right)\right\|_{*} \rightarrow 0$ für $n \rightarrow \infty$ gilt, wobei mit $\|\cdot\|_{*}$ die Operatornorm bezeichnet sei. Gilt zusätzlich $J_{Q, p}\left(v_{n}\right) \rightarrow c \in \mathbb{R}$ für $n \rightarrow \infty$, so heißt $\left(v_{n}\right)_{n}$ eine $(P S)_{c^{-}}$-Folge. Zudem erfülle $J_{Q, p}$ die Palais-Smale Bedingung zum Level $c$, falls jede $(P S)_{c^{-}}$ Folge eine konvergente Teilfolge besitzt. Kompaktheitseigenschaften von $J_{Q, p}$ hängen nun direkt mit Kompaktheitseigenschaften des Birman-Schwinger Operators $\mathbf{K}_{Q, p}: L^{p^{\prime}}\left(\mathbb{R}^{N}\right) \rightarrow$ $L^{p}\left(\mathbb{R}^{N}\right)$ gegeben durch $\mathbf{K}_{Q, p}(f)=Q^{\frac{1}{p}} \mathbf{R}\left(Q^{\frac{1}{p}} f\right)$ zusammen, welche mit Abklingeigenschaften der Gewichtsfunktion $Q$ sowie von Werten von $p$ zusammenhängen. Das folgende Resultat beschreibt diesen Zusammenhang präzise.

Lemma 1.30. [31, Lemma 4.1] Sei $p \in\left[2_{*}, 2^{*}\right)$ sowie $Q \in L^{\infty}\left(\mathbb{R}^{N}\right)$ mit $Q \geq 0$ f.ü. auf $\mathbb{R}^{N}$. Dann ist der Operator

$$
\mathbf{K}_{Q, p}: L^{p^{\prime}}\left(\mathbb{R}^{N}\right) \rightarrow L^{p}\left(\mathbb{R}^{N}\right), \quad \mathbf{K}_{Q, p}(v):=Q^{\frac{1}{p}} \mathbf{R}\left(Q^{\frac{1}{p}} v\right)
$$

symmetrisch in dem Sinne, dass

$$
\int_{\mathbb{R}^{N}} v \mathbf{K}_{Q, p}(w) d x=\int_{\mathbb{R}^{N}} w \mathbf{K}_{Q, p}(v) d x, \quad \text { für alle } v, w \in L^{p^{\prime}}\left(\mathbb{R}^{N}\right) .
$$

Zudem,

(i) $\mathbf{K}_{Q, p}$ ist lokal kompakt, d.h. für jede beschränkte messbare Menge $B \subset \mathbb{R}^{N}$ ist der Operator $\mathbb{1}_{B} \mathbf{K}_{Q, p}$ kompakt. Hier bezeichne $\mathbb{1}_{B}$ die charakteristische Funktion einer messbaren Menge $B \subset \mathbb{R}^{N}$.

(ii) falls $\operatorname{esssup}_{|x| \geq \rho} Q(x) \rightarrow 0$ für $\rho \rightarrow \infty$, so ist $\mathbf{K}_{Q, p}$ kompakt.

Verschwindet also $Q$ bei unendlich, so kann mit Lemma 1.30 gefolgert werden, dass das Funktional $J_{Q, p}$ die Palais-Smale Bedingung erfüllt. Mithilfe eines symmetrischen Mountain-Pass Theorems kann dann die Existenz unendlich vieler Paare kritischer Punkte für $J_{Q, p}$ gefolgert werden. Kombiniert mit nichtlinearen Fernfeld-Relationen lässt sich das komplette Resultat aus [31] wie folgt wiedergeben.

Theorem 1.31. Sei $N \geq 3, p \in\left[2_{*}, 2^{*}\right)$ und $Q \in L^{\infty}\left(\mathbb{R}^{N}\right) \backslash\{0\}$ nicht-negativ und erfülle $\operatorname{esssup}_{|x| \geq R} Q(x) \rightarrow 0$ für $R \rightarrow \infty$. Dann besitzt 1.65 eine unbeschränkte Folge von Paaren $\pm u_{n}$ dualer gebundener Zustandslösungen mit $u_{n} \in W^{2, q}\left(\mathbb{R}^{N}\right) \cap \mathcal{C}^{1, \gamma}\left(\mathbb{R}^{N}\right)$ für alle $q \in[p, \infty)$ 
sowie $\gamma \in(0,1)$ und $n \in \mathbb{N}$. Zudem erfüllen diese Lösungen die nichtlineare Fernfeld-Beziehung

$$
\lim _{R \rightarrow \infty} \frac{1}{R} \int_{B_{R}(0)}\left|u_{n}(x)+2\left(\frac{2 \pi}{|x|}\right)^{\frac{N-1}{2}} \operatorname{Re}\left(e^{i|x|-\frac{i(N-1) \pi}{4}}\right) g_{u_{n}}\left(\frac{x}{|x|}\right)\right|^{2} d x
$$

mit $g_{u_{n}}$ gegeben in (1.64).

Mithilfe von Bemerkung 1.28 sehen wir, dass diese Lösungen auch starke Lösungen sind. Eine weitere Situation, in welcher die Existenz dualer gebundener Zustandslösungen in [31] beweisen wurde ist für periodisches $Q$. In dieser Situation wird $J_{Q, p}$ im Allgemeinen nicht mehr die Palais-Smale Bedingung erfüllen, aber die Existenz einer Palais-Smale Folge zum MountainPass Niveau

$$
c:=\inf _{\gamma \in \Gamma} \sup _{t \in[0,1]} J_{Q, p}(\gamma(t)),
$$

wobei $\Gamma:=\left\{\gamma \in \mathcal{C}\left([0,1], L^{p^{\prime}}\left(\mathbb{R}^{N}\right)\right): \gamma(0)=0, J(\gamma(1))<0\right\}$ sei, lässt sich nachweisen [31, Lemma 6.1] und die Existenz eines kritischen Punktes folgt mithilfe eines Approximationsarguments (siehe [31, Theorem 6.2]). Bemerke hier, dass Lemma $1.29 \Gamma \neq \emptyset$ sowie $c>0$ liefert. Für den Nachweis der Nichttrivialität hilft folgendes „nonvanishing“ -Resultat [31, Theorem 3.1] essentiell.

THEOREM 1.32. Sei $N \geq 3$ sowie $p \in\left(2_{*}, 2^{*}\right)$. Ist $\left(u_{n}\right)_{n} \subset L^{p^{\prime}}\left(\mathbb{R}^{N}\right)$ eine beschränkte Folge mit $\liminf _{n \rightarrow \infty}\left|\int_{\mathbb{R}^{N}} u_{n} \mathcal{R}\left(u_{n}\right) d x\right|>0$, so existieren -nach Übergang zu einer Teilfolge - $R, \zeta>0$ sowie eine Folge $\left(x_{n}\right)_{n} \subset \mathbb{R}^{N}$ mit

$$
\int_{B_{R}\left(x_{n}\right)}\left|u_{n}\right|^{p^{\prime}} d x \geq \zeta>0, \quad \text { für alle } n .
$$

Hier haben wir $\mathcal{R}:=\mathcal{R}_{1}$ gesetzt. Da wir Modifikationen und Verallgemeinerungen dieses Resultats herleiten werden, soll die wesentliche Beweisidee kurz erläutert werden.

Der Beweis basiert auf einer Zerlegung ähnlich zu [37] der Fundamentallösung $\Phi:=\Phi_{1}$ gegeben in 1.54 durch $\Phi=\Phi_{(1)}+\Phi_{(2)}$ mit

$$
\Phi_{(1)}:=\eta * \Phi, \quad \Phi_{(2)}:=\Phi-\Phi_{(1)}
$$

für $\eta \in \mathcal{S}\left(\mathbb{R}^{N}\right)$ so, dass $\widehat{\eta} \in \mathcal{C}_{c}^{\infty}\left(\mathbb{R}^{N}\right)$ mit Werten in $[0,1], \widehat{\eta}=1$ auf $\left\{|| \xi|-1| \leq \frac{1}{6}\right\}$ und $\widehat{\eta}=0$ auf $\left\{|| \xi \mid-1 \geq \frac{1}{4}\right\}$. Die Asymptotik der Hankel-Funktionen liefert nun

$$
|\Phi(x)| \leq C_{0} \max \left\{|x|^{2-N},|x|^{\frac{1-N}{2}}\right\}, \quad \text { für } x \in \mathbb{R}^{N} \backslash\{0\},
$$

mit $C_{0}>0$, und analog zu den Ausführungen nach [31, Theorem 3.1] haben wir

$$
\left|\Phi_{(1)}(x)\right| \leq C_{0}(1+|x|)^{\frac{1-N}{2}}, \quad x \in \mathbb{R}^{N}
$$


sowie

$$
\left|\Phi_{(2)}(x)\right| \leq C_{0} \min \left\{|x|^{2-N},|x|^{-N}\right\}, \quad \text { für } x \in \mathbb{R}^{N} \backslash\{0\} .
$$

Das Theorem 1.32 wird dann für $\Phi_{(1)}, \Phi_{(2)}$ separat gezeigt. Der Nachweis für $\Phi_{(2)}$ ist vergleichsweise kurz. Hier resultiert die Einschränkung $p<2^{*}$. In Bezug auf $\Phi_{(1)}$ ist der wesentliche Schritt den Ausdruck $\left\|\mathbb{1}_{M_{R}} \Phi_{(2)}\right\|_{L^{p}\left(\mathbb{R}^{N}\right)}$ für $M_{R}:=\mathbb{R}^{N} \backslash B_{R}$, wobei $B_{R}$ die (offene) Kugel mit Radius $R$ um den Ursprung bezeichne, mithilfe einer dyadischen Zerlegung abzuschätzen. Für jeden Summanden erhält man dann eine einfache $\left(L^{\infty}, L^{1}\right)$-Abschätzung, sowie mithilfe von Theorem 1.27 ebenfalls eine $\left(L^{2}, L^{\left(2_{*}\right)^{\prime}}\right)$-Abschätzung. Komplexe Interpolation liefert dann eine selbstduale Abschätzung an jeden dyadischen Summanden von $\left\|\mathbb{1}_{M_{R}} \Phi_{(2)}\right\|_{L^{p}\left(\mathbb{R}^{N}\right)}$ mit einem Vorfaktor $A_{j}:=j A(N, p)$, wobei $j \in \mathbb{N}$ der Summationsindex sei. Nun gilt

$$
A(N, p)<0 \Leftrightarrow p>2_{*},
$$

welches notwendig für die Konvergenz der dyadischen Reihe ist. Demzufolge kann der Beweis nur für $p>2_{*}$ vollendet werden.

Wie wir sehen werden (siehe Theorem 2.6), gilt Theorem 1.32 auch für den Sobolev-kritischen Fall $p=2^{*}$ durch Modifikation des $\Phi_{(2)}$ betreffenden Teils. In Kapitel 5 werden wir sehen, dass das Resultat dahingegen für $p=2_{*}$ nicht gilt.

Wir möchten bemerken, dass im Falle einer beschränkten Palais-Smale Folge $\left(u_{n}\right)_{n} \subset L^{p^{\prime}}\left(\mathbb{R}^{N}\right)$ zum Mountain-Pass Niveau $c>0$ die Beziehung

$$
\int_{\mathbb{R}^{N}} Q^{\frac{1}{p}} u_{n} \mathbf{R}\left(Q^{\frac{1}{p}} u_{n}\right) d x=\frac{2 p^{\prime}}{2-p^{\prime}}\left(J_{Q, p}\left(u_{n}\right)-\frac{1}{p^{\prime}} J_{Q, p}^{\prime}\left(u_{n}\right) u_{n}\right) \rightarrow \frac{2 p^{\prime}}{2-p^{\prime}} c>0
$$

für $n \rightarrow \infty$ gilt. Diese Folge erfüllt also die Voraussetzungen von Theorem 1.32, Der Beweis der Nichttrivialität des kritischen Punktes erhält man dann aus einer lokalen Konvergenzaussage ausgehend von 1.72) (siehe auch [31, Theorem 6.2]). Das vollständige Resultat für periodische Gewichtsfunktionen lautet wie folgt.

Theorem 1.33. Sei $N \geq 3$ sowie $p \in\left(2_{*}, 2^{*}\right)$ und sei $Q \in L^{\infty}\left(\mathbb{R}^{N}\right) \backslash\{0\}$ nicht-negativ sowie $\mathbb{Z}^{N}$ periodisch. Dann besitzt (1.47) eine nicht-triviale gebundene Zustandslösung, welche zusätzlich 1.70 mit $g_{u}$ anstelle von $g_{u_{n}}$ erfüllt.

3.5. Weitere sowie verwandte Resultate. Für den Fall räumlich kompakt getragener Nichtlinearitäten $f$ wurde in [30] Existenz sowie Vielfachheit von Lösungen des Problems

$$
-\Delta u-k^{2} u=f(x, u), \quad x \in \mathbb{R}^{N}
$$

beweisen. Mit einer Dirichlet-zu-Neumann Abbildung assoziiert mit dem Außenraumproblem, kann das Problem reduziert werden zur Existenz kritischer Punkte eines $H^{1}$-Funktionals, welches auf einem Ball um den Ursprung definiert ist. Diese Methoden basieren stark auf der Lokalisation des Trägers von $f$. In [27] wird ein dualer Rahmen in Orlicz-Räumen erarbeitet, 
welcher ebenfalls allgemeinere Nichtlinearitäten zulässt. Zudem werden hier, unter zusätzlichen Abklingbedingungen an die Nichtlinearität $f$, ebenfalls Vielfachheitsresultate sowie FernfeldRelationen hergeleitet. In [29] wird die Vielfachheit von Lösungen im Falle periodischer Gewichtsfunktionen bewiesen, wobei Ideen im Schrödinger-Kontext aus [64] erfolgreich in die Methode eingebettet werde konnten. Zudem wurde die Existenz von Lösungen im Falle asymptotisch periodischer $Q$ nachgewiesen.

Es soll erwähnt sein, dass die duale variationelle Methode für die Helmholtz-Gleichung in [52] auch auf den Fall $Q \leq 0$ übertragen wurde. Zudem haben die Autoren die Existenz radialer Lösungen von 1.75) im Fall radialer $f$ für Werte $p>\frac{2 N}{N-1}$ etabliert. In [32] wird die Helmholtz-Gleichung vom Standpunkt der Bifurkationstheorie beleuchtet. Genauer wird die Existenz stetiger Lösungszweige für (1.47) in Abhängigkeit des Parameters $\lambda=-k^{2}$ untersucht und gezeigt, dass dies auch Parameterwerte innerhalb des wesentlichen Spektrums von $-\Delta$ zulässt. Der Fall $N=2$ für (1.47) wird in [26] erfolgreich untersucht. In [15] werden komplexe Lösungen für die nichtlineare Helmholtz-Gleichung für allgemeine Nichtlinearitäten hergeleitet. Die Autoren haben keine duale Formulierung oder Maximumprinzipien zur Verfügung und analysieren die Gleichung (1.61) stattdessen mithilfe von Fixpunktmethoden sowie globalen Bifurkationsmethoden. Zudem seien die Arbeiten [21,,[53],[54] im Kontext von HelmholtzSystemen erwähnt.

3.6. Hauptresultate der vorliegenden Arbeit. Den Umfang der Forschungsleistung bilden die Arbeiten [Y1, [Y2] sowie [Y3 welche wir nun zusammenfassen und einordnen möchten.

Duale Grundzustandslösungen der kritischen nichtlinearen Helmholtz - Gleichung im Sobolev-kritischen Fall. Dieser erste vorgestellte Artikel [Y1] ist eine Kollaboration mit Gilles Evèquoz und befasst sich mit der Helmholtz-Gleichung im Sobolev-kritischen Fall $p=2^{*}$, also

$$
-\Delta u-u=Q(x)|u|^{2^{*}-2} u, \quad \text { in } \mathbb{R}^{N}
$$

für $N \geq 3$ sowie $Q \in L^{\infty}\left(\mathbb{R}^{N}\right) \backslash\{0\}$ nicht-negativ.

Wie bereits in Lemma 1.30 erwähnt, ist der Birman-Schwinger Operator $\mathbf{K}_{Q}:=\mathbf{K}_{Q, 2^{*}} L^{2^{+}}\left(\mathbb{R}^{N}\right) \rightarrow$ $L^{2^{*}}\left(\mathbb{R}^{N}\right)$, wobei $2^{+}:=\left(2^{*}\right)^{\prime}=\frac{2 N}{N+2}$ gegeben durch

$$
\mathbf{K}_{Q}(f)=Q^{\frac{1}{p}} \mathbf{R}\left(Q^{\frac{1}{p}} f\right)
$$

nicht einmal lokal kompakt, was an der Nicht-Kompaktheit der dem Lemma zugrunde liegenden Sobolev-Einbettung liegt. Wir zeigen, dass die Palais-Smale Bedingung für das duale 
Funktional $\left.J_{Q}:=J_{Q, 2^{*}}: L^{2^{+}}\left(\mathbb{R}^{N}\right) \rightarrow L^{2^{*}}\left(\mathbb{R}^{N}\right)\right)$ gegeben durch

$$
J_{Q}(u)=\frac{1}{2^{+}}\|u\|_{L^{2^{+}}\left(\mathbb{R}^{N}\right)}^{2^{+}}-\frac{1}{2} \int_{\mathbb{R}^{N}} u \mathbf{K}_{Q}(u) d x
$$

zumindest für gewisse Energieniveaus erhalten werden kann. Hierzu vergleichen wir das MountainPass Niveau $L_{Q}$ von $J_{Q}$ mit dem geringsten Energieniveau $L_{Q}^{*}$ aller assoziierten Grenzprobleme

$$
-\Delta u=Q\left(x_{0}\right)|u|^{p-2} u, \quad \text { in } \mathbb{R}^{N}
$$

für $x_{0} \in \mathbb{R}^{N}$, ähnlich zur klassischen Arbeit [10] von Brézis und Nirenberg. Hierzu stellen wir fest, dass

$$
L_{Q}^{*}=\frac{S^{\frac{N}{2}}}{N\|Q\|_{\infty}^{\frac{N-2}{2}}},
$$

wobei $S$ die optimale Konstante aus der Sobolev-Einbettung (siehe [46, §8]) bezeichne. Unser Ansatz zum Auffinden kritischer Punkte von $J_{Q}$ kann also mithilfe der folgenden zwei Schritte zusammengefasst werden:

(I) Zeige, dass die Palais-Smale Bedingung für Niveaus $0<\beta<L_{Q}^{*}$ erfüllt ist.

(II) Zeige, dass tatsächlich $L_{Q}<L_{Q}^{*}$ gilt.

Wir vergleichen die Gleichungen (1.76) und (1.77) auf der Ebene der Fundamentallösungen (und Resolventen), da wir für (1.76) kein direktes Funktional zur Verfügung haben. Im folgenden Lemma leiten wir eine Abschätzung für die Differenz der Fundamentallösungen $\Psi-\Lambda: \mathbb{R}^{N} \backslash$ $\{0\} \rightarrow \mathbb{R}$, wobei $\Psi=\Psi_{1}$ die Fundamentallösung zu 1.76 und $\Lambda$ die Fundamentallösung $\mathrm{zu}$ (1.77) ist, ausgewertet auf kleinen Mengen her. Dieses Resultat erweist sich ebenfalls im zweiten Schritt als essentiell.

Lemma 1.34. Sei $r>0$ so, dass $r<y_{\frac{N-4}{2}}$ falls $N \geq 4$, wobei $y_{\frac{N-4}{2}}$ die erste positive Nullstelle der Bessel-Funktion $Y_{\frac{N-4}{2}}$ sei, und sei $r<\pi$ falls $N=3$.

(i) Es existieren $\kappa_{1}, \kappa_{2}>0$ nur abhängig von $r$ und $N$, sodass für alle $x \in B_{r}(0)$,

$$
\begin{cases}\kappa_{1}|x|^{4-N} \leq \Psi(x)-\Lambda(x) \leq \kappa_{2}|x|^{4-N}, & \text { für } N \geq 5, \\ \kappa_{1}|\ln | x|| \leq \Psi(x)-\Lambda(x) \leq \kappa_{2}|\ln | x||, & \text { für } N=4, \\ -\kappa_{1}|x| & \geq \Psi(x)-\Lambda(x) \geq-\kappa_{2}|x|, \quad \text { für } N=3 .\end{cases}
$$

(ii) Für jeden Multiindex $\alpha \in \mathbb{N}_{0}^{N}$ mit $|\alpha| \geq 1$, existiert ein $\kappa_{3}>0$ nur abhängig von $|\alpha|$, $r$ und $N$, sodass

$$
\left|\partial^{\alpha}(\Psi(x)-\Lambda(x))\right| \leq \kappa_{3}|x|^{4-N-|\alpha|}, \quad \text { für alle } x \in B_{r}(0) .
$$

Der Beweis dieser Behauptung basiert auf präzisen Abschätzungen von Bessel-Funktionen für kleine Argumente. Eine erste Konsequenz dieses Lemmas ist die Kompaktheit des Operators 
$\mathbf{R}-\mathbf{R}_{0}: L^{2^{+}}\left(\mathbb{R}^{N}\right) \rightarrow L_{l o c}^{2^{*}}\left(\mathbb{R}^{N}\right)$, wobei $\mathbf{R}_{0}: f \mapsto \Lambda * f$ den Resolventenoperator zur LaplaceGleichung bezeichne.

Proposition 1.35.

(i) Die Differenz $\mathbf{R}-\mathbf{R}_{0}$ ist eine stetige lineare Abbildung von $L^{2^{+}}\left(\mathbb{R}^{N}\right)$ nach $W^{2,2^{*}}\left(\mathbb{R}^{N}\right)$

(ii) Für alle $r>0$, ist der Operator $\mathbb{1}_{B_{r}}\left(\mathbf{R}-\mathbf{R}_{0}\right): L^{2^{+}}\left(\mathbb{R}^{N}\right) \rightarrow L^{2^{*}}\left(\mathbb{R}^{N}\right)$ kompakt.

Zusammen mit der Ergebnis, dass $\mathbf{R}: L^{2^{+}}\left(\mathbb{R}^{N}\right) \rightarrow L^{t}\left(\mathbb{R}^{N}\right)$ für subkritische $t \in\left[1,2^{*}\right)$ lokal kompakt ist, können wir den ersten Schritt vollenden. Indem wir zusätzlich zeigen, dass das nonvanishing Resultat aus Theorem 1.32 auch im Fall $p=2^{*}$ gilt, können wir auch periodische Gewichtsfunktionen in unser Resultat miteinbeziehen. Unser Hauptresultat in Bezug auf Schritt (I) lautet wie folgt.

Proposition 1.36. Sei $Q \in L^{\infty}\left(\mathbb{R}^{N}\right) \backslash\{0\}$ von der Form $Q=Q_{0}+Q_{\text {per }}$ mit $Q_{0}, Q_{\text {per }} \geq 0$ derart, dass $Q_{0}(x) \rightarrow 0$ für $|x| \rightarrow \infty$ sowie $Q_{\text {per }}$ eine $\mathbb{Z}^{N}$-periodische Funktion ist.

Ist nun $\left(v_{n}\right)_{n} \subset L^{2^{+}}\left(\mathbb{R}^{N}\right)$ eine Palais-Smale Folge für $J_{Q}$ zum Level $\beta=L_{Q}<L_{Q}^{*}$, so existiert ein nicht-trivialer kritischer Punkt $w \in L^{2^{+}}\left(\mathbb{R}^{N}\right)$ von $J_{Q}$ mit $J_{Q}(w)=L_{Q}$.

Um das Mountain-Pass Niveau $L_{Q}$ abzuschätzen, bemerken wir die in unserer Situation alternative Beschreibung

$$
L_{Q}=\inf \left\{\frac{1}{N}\left(\frac{\int_{\mathbb{R}^{N}}|v|^{2^{+}} d x}{\int_{\mathbb{R}^{N}} v \mathbf{K}_{Q} v d x}\right)^{\frac{N}{2}}: v \in L^{2^{+}}\left(\mathbb{R}^{N}\right) \operatorname{mit} \int_{\mathbb{R}^{N}} v \mathbf{K}_{Q} v d x>0\right\},
$$

aus welcher wir folgern können, dass

$$
L_{Q}=\inf \left\{J_{Q}(v): v \in L^{2^{+}}\left(\mathbb{R}^{N}\right) \text { with } J_{Q}^{\prime}(v)=0\right\}
$$

gilt. Die Lösungen, welche wir erhalten sind duale Grundzustandslösungen, also Lösungen $u \in L^{2^{*}}\left(\mathbb{R}^{N}\right)$ der Form $u=\mathbf{R}\left(Q^{\frac{1}{2^{+}}} v\right)$, wobei $v \in L^{2^{+}}\left(\mathbb{R}^{N}\right)$ kritischer Punkt von $J_{Q}$ mit $J_{Q}(v)=L_{Q}$ ist, demzufolge also die minimale Energie unter allen kritischen Punkt haben. Nach Bemerkung 1.28 sind diese Lösungen starke Lösungen von (1.76). Um Schritt (II) nachzuvollziehen, schätzen wir die Ausdrücke

$$
\frac{\left\|v_{\varepsilon, \alpha}\right\|_{2^{+}}^{2^{+}}}{\int_{\mathbb{R}^{N}} v_{\varepsilon, \alpha} \mathbf{K}_{Q} v_{\varepsilon, \alpha} d x}
$$

wobei $v_{\varepsilon, \alpha} \in L^{2^{+}}\left(B_{2 \alpha}(0)\right)$ glatt abgeschnittene Versionen von Optimierern $v_{\varepsilon} \in L^{2^{+}}\left(\mathbb{R}^{N}\right)$ der Hardy-Littlewood-Sobolev-Ungleichung sind, also

$$
\int_{\mathbb{R}^{N}} v_{\varepsilon} \mathbf{R}_{0} v_{\varepsilon} d x=S^{-1}\left\|v_{\varepsilon}\right\|_{2^{+}}^{2^{+}}
$$


welche (bis auf Translationen und skalare Vielfache) gegeben sind durch

$$
v_{\varepsilon}(x)=(N(N-2) \varepsilon)^{\frac{N+2}{4}}\left(\frac{1}{\varepsilon+|x|^{2}}\right)^{\frac{N+2}{2}}, \quad \varepsilon>0 .
$$

Auch in diesem Schritt ist Lemma 1.34 essentiell und der Vorzeichenunterschied in Dimensionen $N \geq 4$ und $N=3$ zeigt sich hier deutlich. Während wir $L_{Q}<L_{Q}^{*}$ für $N \geq 4$ beweisen können, haben wir im Fall $N=3$ tatsächlich die Gleichheit $L_{Q}=L_{Q}^{*}$. Zusätzlich zeigen wir, dass das Mountain-Pass Niveau in diesem Fall nicht angenommen wird und erhalten ein Nichtexistenz Resultat für unseren Ansatz. Unser Hauptresultat ist wie folgt.

Theorem 1.37. Sei $N \geq 3$ und $Q \in L^{\infty}\left(\mathbb{R}^{N}\right) \backslash\{0\}$ mit $Q \geq 0$ fast überall auf $\mathbb{R}^{N}$.

(i) Falls $N \geq 4$ und $Q$ erfüllt die Bedingungen

(Q1) $Q=Q_{0}+Q_{\text {per }}$, wobei $Q_{0}, Q_{\text {per }} \geq 0$ derart sind, dass $Q_{\text {per }}$ eine $\mathbb{Z}^{N}$-periodische Funktion ist und $Q_{0}(x) \rightarrow 0$ für $|x| \rightarrow \infty$.

(Q2) Es existiert $x_{0} \in \mathbb{R}^{N}$ mit $Q\left(x_{0}\right)=\max _{\mathbb{R}^{N}} Q$ und für $\left|x-x_{0}\right| \rightarrow 0$ gilt

$$
Q(x)-Q\left(x_{0}\right)= \begin{cases}o\left(\left|x-x_{0}\right|^{2}\right), & \text { falls } N \geq 5 \\ O\left(\left|x-x_{0}\right|^{2}\right), & \text { falls } N=4\end{cases}
$$

Dann hat 1.76) eine duale Grundzustandslösung.

(ii) Ist $N=3$, dann existiert keine duale Grundzustandslösung für (1.76).

Die Bedingung (Q2) ist hinreichend um $L_{Q}<L_{Q}^{*}$ im Fall $N \geq 4$ nachzuweisen. Da $Q$ im Allgemeinen nicht konstant ist, benötigen wir eine Bedingung, welche beschreibt mit welcher Rate $Q$ den Wert $\|Q\|_{\infty}$ erreicht. Diese Bedingung erscheint in diversen kritischen Problemen (siehe z.B. [25]) und wurde auch von Chabrowski und Szulkin in [14] im Kontext kritischer Schrödingergleichungen verwendet. In [24 sind Beispiele kritischer Probleme aufgezählt, für welche (Q2) auch notwendig ist.

Gewichtete Fourier-Fortsetzung Resultate sowie G-invariante Lösungen für die nichtlineare Helmholtz-Gleichung. Grundlage dieser Arbeit ist der Artikel [Y2], welcher aus einer Kollaboration mit Tobias Weth resultiert. Hier befassen wir uns mit der Existenz (nicht radialer) reellwertiger Lösungen der Helmholtz-Gleichung

$$
-\Delta u-u=Q(x)|u|^{p-2} u, \quad \text { in } \mathbb{R}^{N}
$$

mit $Q \in L^{\infty}\left(\mathbb{R}^{N}\right) \backslash\{0\}$ und mögliche Werte $p \in\left(2,2^{*}\right)$. Diesbezüglich studieren wir Implikationen verbesserter Fourier-Fortsetzungsresultate in Bezug auf Resolventenabschätzungen sowie nonvanishing Aussagen. Zudem leiten wir eine explizite verbesserte Fourier - Fortsetzungsabschätzungen her und untersuchen die Konsequenzen im Hinblick auf die duale variationelle Formulierung von 1.79 . 
Motiviert dadurch, dass Fourier-Fortsetzungsabschätzungen der Form (1.57) sowohl im Hinblick auf Symmetrien als auch durch gewichtete Normen verbessert werden können, definieren wir für $q \geq 1, G \subset O(N)$ eine abgeschlossene Untergruppe und eine messbare Funktion $Q$ : $\mathbb{R}^{N} \rightarrow \mathbb{C}$ das zulässige Fortsetzungstripel $(G, q, Q)$. Dies ist ein Tripel $(G, q, Q)$, für welches die (gewichtete) Fourier-Fortsetzungsungleichung

$$
\left\|Q \check{F}_{\sigma}\right\|_{L^{p}\left(\mathbb{R}^{N}\right)} \leq C\|F\|_{L^{2}\left(\mathbb{S}^{N-1}\right)}, \quad \text { für alle G-invarianten Funktionen } F \in L^{2}\left(\mathbb{R}^{N}\right)
$$

mit einer von $F$ unabhängigen Konstanten $C>0$ gilt. Hierbei nennen wir $F \in L^{2}\left(\mathbb{S}^{N-1}\right)$ eine $G$-invariante Funktion, falls $F=F(A \cdot)$ für alle $A \in G$ gilt. Gemäß Theorem 1.26 ist demnach das Tripel $(\{\mathrm{id}\}, q, 1)$ ein zulässiges Fourier-Fortsetzungstripel für alle $q \geq 2 *$ und $(O(N), q, 1)$ ein zulässiges Fourier-Fortsetzungstripel bereits für alle $q>\frac{2 N}{N-1}$. Ist $Q \in L^{s}\left(\mathbb{R}^{N}\right)$ für $s \in[1, \infty)$ so liefert eine Anwendung der Hölderschen Ungleichung in $(1.80)$, dass $(\{\operatorname{id}, q, Q\})$ ein zulässiges Fourier-Fortsetzungstripel für alle $q \geq \max \{1, L(s, N)\}$ ist, wobei $L(s, N):=\frac{2(N+1)}{(N-1)+\frac{2}{s}(N+1)}$ von unten für $s \rightarrow \infty$ gegen $2_{*}$ konvergiert. Im folgenden interessieren wir uns für zulässige Fortsetzungstripel mit $Q \in L^{\infty}\left(\mathbb{R}^{N}\right)$, bei welchen wir keine Verbesserung durch das Abklingverhalten der Gewichtsfunktion erzielen, sondern durch die betrachteten Symmetrien.

Hierzu betrachten wir konkret $G_{k}:=O(N-k) \times O(k)$ für $k \in\{1,2, \ldots, N-1\}$ sowie $Q=$ $Q_{\alpha}:=\mathbb{1}_{L_{\alpha}}$ mit

$$
L_{\alpha}:=\left\{x=\left(x^{(N-k)}, x^{(k)}\right) \in \mathbb{R}^{N-k} \times \mathbb{R}^{k}:\left|x^{(N-k)}\right| \leq a\left|x^{(k)}\right|^{-\alpha}\right\} .
$$

Wegen $\left|L_{\alpha}\right|=\infty$ für alle $\alpha>0$ ist $Q_{\alpha}:=\mathbb{1}_{L_{\alpha}} \notin L^{s}\left(\mathbb{R}^{N}\right)$ für alle $s \in[1, \infty)$ sowie $\alpha>0$. Unser Resultat lautet wie folgt.

Theorem 1.38. Seien $N \geq 3, k \in\{1, \ldots, N-1\}, \alpha>0$, und setze $Q_{\alpha}=\mathbb{1}_{L_{\alpha}}$ mit $L_{\alpha}$ gegeben in (1.81. Nehme zudem an, dass

$$
\alpha>\frac{1}{N-1} \quad \text { falls } k=1, \quad \alpha<N-1 \quad \text { falls } k=N-1,
$$

und setze

$$
\lambda_{N, k, \alpha}:= \begin{cases}\frac{2(N-1)-\frac{2}{\alpha}}{N-2}, & \text { falls } k=1 ; \\ \max \left\{\frac{2(N-k)-\frac{2 k}{\alpha}}{N-k-1}, \frac{2 k-2 \alpha(N-k)}{k-1}\right\} & \text { falls } 2 \leq k \leq N-2 ; \\ \frac{2(N-1)-2 \alpha}{N-2}, & \text { falls } k=N-1 .\end{cases}
$$

Dann ist $\left(G_{k}, q, Q_{\alpha}\right)$ ein zulässiges Fourier-Fortsetzungstripel für jedes $q>\lambda_{N, k, \alpha}$.

Tatsächlich werden wir eine Verallgemeinerung beweisen, indem wir Mengen der Form

$$
L_{\alpha, \beta}:=\left\{x=\left(x^{(N-k)}, x^{(k)}\right) \in \mathbb{R}^{N-k} \times \mathbb{R}^{k}:\left|x^{(N-k)}\right| \leq a \max \left\{\left|x^{(k)}\right|^{-\alpha},\left|x^{(k)}\right|^{-\beta}\right\}\right\}
$$


für $\alpha \geq \beta>0$ betrachten. Eine genauere Betrachtung von $\lambda_{N, k, \alpha}$ zeigt, dass auch Werte $q \leq 2_{*}$ realisierbar sind. Der Verlauf zulässiger Werte von $q$ ist beispielhaft mithilfe folgender Grafik veranschaulicht.

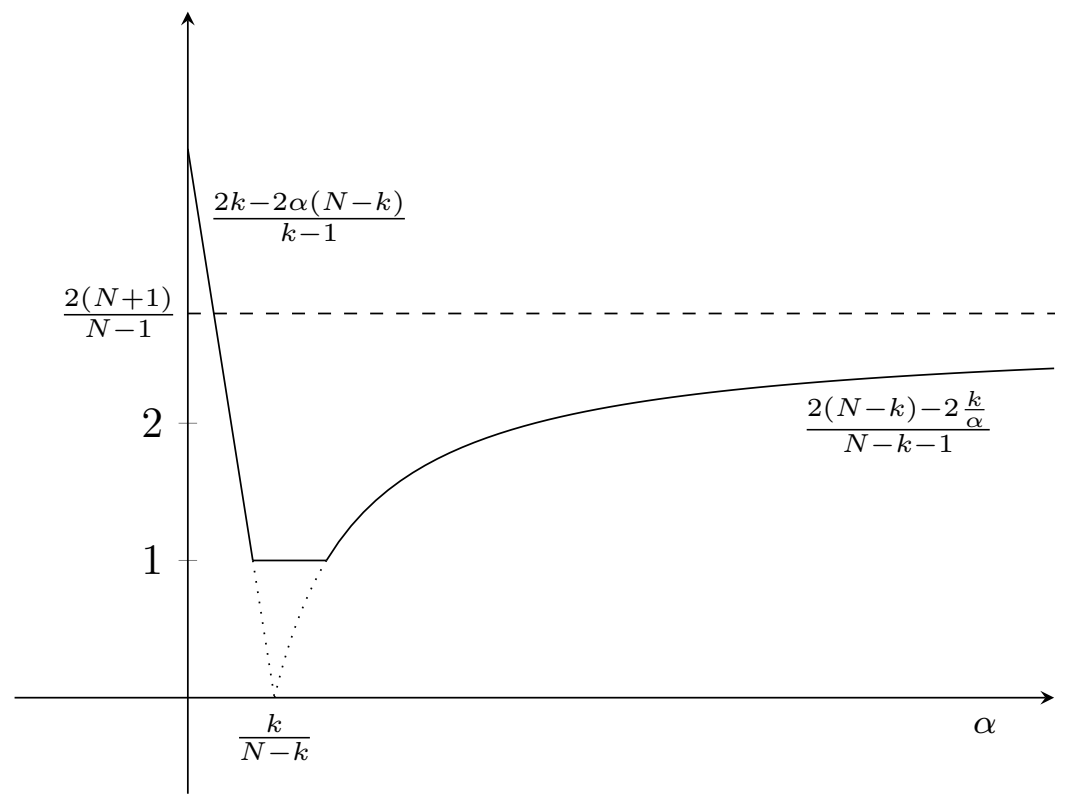

FiguRE 2. Veranschaulichung zulässiger Werte für $q$ am Beispiel $N=6$ und $k=2$ in Abhängigkeit von $\alpha$

Der Beweis von Theorem 1.38 wird in zwei Schritten vollzogen. Als ersten Schritt verwenden wir die Symmetrien und schreiben $F \in L^{2}\left(\mathbb{S}^{N-1}\right)$ um zu einer Funktion $h_{F}$ auf $[0,1]$ gegeben durch

$$
h_{F}:[0,1] \rightarrow \mathbb{R}, \quad h_{F}(r):=F\left(r \eta, \sqrt{1-r^{2}} \mu\right), \quad \text { für } \eta \in \mathbb{S}^{N-k-1}, \mu \in \mathbb{S}^{k-1} .
$$

Dann reduziert sich $\|Q \check{F}\|_{L^{q\left(\mathbb{R}^{N}\right)}}$ zu einem eindimensionalen Integral, in welchem die Größen $d \check{\sigma}_{N-k}$ und $d \check{\sigma}_{k}$ vorkommen. In Bezug auf die Abschätzung dieses Integrals nimmt dann 1.59 eine zentrale Rolle ein.

Anschließend studieren wir abstrakte Implikationen für zulässige Fortsetzungstripel im Hinblick auf Resolventenabschätzungen und nonvanishing Resultate. Speziell zeigen wir, dass im Falle eines zulässigen Fortsetzungstripels $(G, q, Q)$ für $G$-invariantes $Q \in L^{\infty}\left(\mathbb{R}^{N}\right)$, wir bezüglich des gewichteten Resolventenoperators $\mathcal{R}_{Q}: f \mapsto Q \Phi *(Q f)$ selbstduale Abschätzungen sowie nonvanishing Resultate für alle $p \in\left(\frac{2 N}{N-1} \frac{2 q}{q+2}, 2^{*}\right]$ erhalten. Bemerke, dass $p<2 *$ äquivalent zu $\frac{2 N}{N-1} \frac{2 q}{q+2}<2 *$ ist. 
Theorem 1.39. Sei $N \geq 3, G \subset O(N)$ eine abgeschlossene Untergruppe, $Q \in L^{\infty}\left(\mathbb{R}^{N}\right) \backslash$ $\{0\}$ sei $G$-invariant und sei $q \in\left[1, \frac{2(N+1)}{N-1}\right]$ derart, dass $(G, Q, q)$ ein zulässiges FourierFortsetzungstripel ist. Dann existiert für alle $p \in\left(\frac{2 N}{N-1} \frac{2 q}{q+2}, 2^{*}\right]$ eine Konstante $C>0$ mit

$$
\left\|\mathcal{R}_{Q}(f)\right\|_{L^{p}\left(\mathbb{R}^{N}\right)} \leq C\|f\|_{L^{p^{\prime}\left(\mathbb{R}^{N}\right)}}
$$

für alle $G$-invarianten Schwartz-Funktionen $f \in \mathcal{S}\left(\mathbb{R}^{N}\right)$.

Wir werden Theorem 1.39 in allgemeinerer nicht-selbstdualer Form beweisen (siehe Theorem 3.12 in Kapitel 3) aus welchem Theorem 1.39 dann als Spezialfall hervorgeht. Die Strategie hierzu ist ähnlich zu [37, [43] siehe auch [31]. Unter den selben Voraussetzungen wie in Theorem 1.39 erhalten wir die Gültigkeit eines nonvanishing Theorems.

Theorem 1.40. Sei $N \geq 3, G \subset O(N)$ eine abgeschlossene Untergruppe, $Q \in L^{\infty}\left(\mathbb{R}^{N}\right) \backslash$ $\{0\}$ sei $G$-invariant und sei $q \in\left[1, \frac{2(N+1)}{N-1}\right]$ derart, dass $(G, Q, q)$ ein zulässiges FourierFortsetzungstripel ist. Zudem sei $p \in\left(\frac{2 N}{N-1} \frac{2 q}{q+2}, 2^{*}\right]$ Dann gilt für jede beschränkte und $G$-invariante Folge $\left(v_{n}\right)_{n} \subset L^{p^{\prime}}\left(\mathbb{R}^{N}\right)$, welche

$$
\left|\limsup _{n \rightarrow \infty} \int_{\mathbb{R}^{N}} v_{n} \mathcal{R}_{Q}\left(v_{n}\right) d x\right|>0
$$

erfüllt, dass - nach Übergang zu einer Teilfolge - Zahlen $R, \zeta>0$ sowie eine Folge $\left(x_{n}\right)_{n} \subset \mathbb{R}^{N}$ existieren mit

$$
\int_{B_{R}\left(x_{n}\right)}\left|v_{n}(x)\right|^{p^{\prime}} d x \geq \zeta>0, \quad \text { für alle } n .
$$

Es sei bemerkt, dass wir für $G=\{\operatorname{id}\}$ und $Q \equiv 1$ exakt die Aussage aus Theorem 1.32 erhalten, da $\frac{2 N}{N-1} \frac{2 q}{q+2}=2_{*}$ für $q=2_{*}$ gilt.

Mithilfe der Theoreme 1.39 und 1.40 sind wir nun in der Lage erste Existenzresultate in Bezug auf das Problem (1.79) zu formulieren.

Theorem 1.41. Sei $N \geq 3$ und $G \subset O(N)$ eine abgeschlossene Untergruppe und sei $Q \in$ $L^{\infty}\left(\mathbb{R}^{N}\right) \backslash\{0\}$ G-invariant, nicht-negativ und erfülle

$$
\int_{B_{R}(x)} Q(x) d x \rightarrow 0, \quad \text { für }|x| \rightarrow \infty \text { für ein } R>0 .
$$

Zudem seien $q \in\left[1, \frac{2(N+1)}{N-1}\right]$ und $p \in\left(\max \left\{\frac{2 N}{N-1} \frac{2 q}{q+2}, 2\right\}, 2^{*}\right)$ derart, dass $\left(G, q, Q^{\frac{1}{p}}\right)$ ein zulässiges Fourier-Fortsetzungstripel ist. Dann besitzt 1.79 eine nichttriviale G-invariante duale gebundene Zustandslösung.

Hier sei angemerkt, dass $p>2$ im Hinblick auf den Erhalt der Mountain-Pass Geometrie gewährleistet werden muss. 
Nach Theorem 1.26 ist $\left(G, q, Q^{\prime}\right)$ ein zulässiges Fourier-Fortsetzungstripel für $q=2_{*}$, jede abgeschlossene Untergruppe $G \subset O(N)$ und jedes $Q^{\prime} \in L^{\infty}\left(\mathbb{R}^{N}\right)$. Da zudem $\frac{2 N}{N-1} \frac{2 q}{q+2}=2_{*}$ für $q=2_{*}$ gilt, haben wir die folgende direkte Konsequenz aus Theorem 1.41 .

Korollar 1.42. Sei $N \geq 3, G \subset O(N)$ eine abgeschlossene Untergruppe und sei $Q \in$ $L^{\infty}\left(\mathbb{R}^{N}\right) \backslash\{0\}$ eine nicht-negative und $G$-invariante Funktion, welche 1.85 erfüllt. Dann besitzt (1.79) eine nicht-triviale duale gebundene Zustandslösung für $p \in\left(2_{*}, 2^{*}\right)$.

Dieses Korollar gilt insbesondere im nicht-symmetrischen Fall $G=\{$ id $\}$, aber benötigt die Abklingbedingung (1.85). Im speziellen Fall $G=G_{k}=O(N-k) \times O(k) \subset O(N)$ können wir 1.85 fallen lassen um Lösungen im nicht-kritischen Fall $p \in\left(2_{*}, 2^{*}\right)$ zu erhalten.

Theorem 1.43. Sei $N \geq 4, k \in\{2,3, \ldots, N-2\}$ und sei $Q \in L^{\infty}\left(\mathbb{R}^{N}\right) \backslash\{0\}$ nicht-negativ und $G_{k}$-invariant. Dann besitzt 1.79 eine $G_{k}$-invariante duale gebundene Zustandslösung für $p \in\left(2_{*}, 2^{*}\right)$.

Die Bedingung an $N$ und $k$ ist hier notwendig um zu gewährleisten, dass die minimale Orbitdimension von $G_{k}$ gleich 1 ist. Dies wird für den Nachweis der Beschränktheit der aus dem nonvanishing Theorem 1.40 resultierenden Folge von Punkten $\left(x_{n}\right)_{n}$ benötigt. Kombination des nonvanishing Resultats mit der Symmetrie liefert einen Widerspruch zur Beschränktheit der betrachteten Palais-Smale Folge im Falle unbeschränkter $\left(x_{n}\right)_{n} \subset \mathbb{R}^{N}$.

Zuletzt möchten wir hervorheben, dass die Bedingung für die Konkrete Wahl $Q=Q_{\alpha}=\mathbb{1}_{L_{\alpha}}$ und Konsequenterweise für jede messbare Funktion $|\tilde{Q}| \leq c \mathbb{1}_{L_{\alpha}}$ erfüllt ist. Zudem ist für ein zulässiges Fortsetzungstripel $(G, q, Q)$, demnach auch $(G, q, \tilde{Q})$ eines. Hiermit haben wir folgendes Korollar als Konsequenz von Theorem 1.38 sowie Theorem 1.41 .

Korollar 1.44. Sei $N \geq 3, k \in\{1, \ldots, N-1\}$, und sei $\alpha>0$. Zudem sei $Q \in L^{\infty}\left(\mathbb{R}^{N}\right)$ nicht-negativ und $G_{k}$-invariant mit $Q \not \equiv 0$ sowie $|Q| \leq c \mathbb{1}_{L_{\alpha}}$ für ein $c>0$. Dann besitzt 1.79 eine nicht-triviale, $G_{k}$-invariante gebundene Zustandslösung für alle $p \in\left(\mu_{N, k, \alpha}, \frac{2 N}{N-2}\right)$, falls

(i) $k=1$ und

$$
\mu_{N, 1, \alpha}:= \begin{cases}2, & \frac{1}{N-1}<\alpha \leq \frac{N+1}{3(N-1)}, \\ \frac{4 N(\alpha(N-1)-1)}{(N-1)(2 \alpha N-3 \alpha-1)}, & \alpha>\frac{N+1}{3(N-1)} .\end{cases}
$$

(ii) $k=N-1$ und

$$
\mu_{N, N-1, \alpha}:= \begin{cases}\frac{4 N(N-1-\alpha)}{(N-1)(2 N-\alpha-3)}, & 0<\alpha \leq \frac{3(N-1)}{N+1}, \\ 2, & \frac{3(N-1)}{N+1}<\alpha<N-1 .\end{cases}
$$


(iii) $2 \leq k \leq N-2$ und

$$
\mu_{N, k, \alpha}:= \begin{cases}\frac{4 N(k-\alpha(N-k))}{(N-1)(2 k-1-\alpha(N-k))}, & \alpha \leq \frac{N+2 k-1}{(N+1)(N-k)}, \\ 2, & \frac{N+2 k-1}{(N+1)(N-k)}<\alpha \leq \frac{(N+1) k}{N-1+2(N-k)}, \\ \frac{4 N(\alpha(N-k)-k)}{(N-1)(2 \alpha(N-k)-\alpha-k)}, & \alpha>\frac{(N+1) k}{N-1+2(N-k)} .\end{cases}
$$

Duale variationelle Methoden für eine nichtlineare Helmholtz-Gleichung mit vorzeichenwechselnder Nichtlinearität. Die dritte Arbeit [Y3], welche aus einer Kollaboration mit Rainer Mandel und Dominic Scheider resultiert, befasst sich mit der Etablierung eines dualen variationellen Rahmens für

$$
-\Delta u-k^{2} u=Q|u|^{p-2} u, \quad x \in \mathbb{R}^{N}, p \in\left[2_{*}, 2^{*}\right], k>0
$$

im Falle vorzeichenwechselnder Gewichtsfunktionen $Q \in L^{\infty}\left(\mathbb{R}^{N}\right)$. Neben dem etablierten Fall $Q \geq 0$ aus [31], haben die Autoren in [52] die Anwendbarkeit der dualen Methode auch für $Q \leq 0$ demonstriert. Ausgehend von der Integralgleichung $u=\mathbf{R}_{k}\left(Q|u|^{p-2} u\right)$ für $u \in L^{p}\left(\mathbb{R}^{N}\right)$ betrachten sie dort die duale Transformation $v:=|Q|^{\frac{1}{p}}|u|^{p-2} u$ welche

$$
|v|^{p^{\prime}-2} v=-|Q|^{\frac{1}{p^{\prime}}} \mathbf{R}_{k}\left(|Q|^{\frac{1}{p^{\prime}}}|u|^{p-2} u\right)
$$

liefert und eine variationelle Struktur trägt (siehe [52, §3 ]). Es soll bemerkt sein, dass die rechte Seite der dualen Gleichung (1.90) ein negatives Vorzeichen wegen $Q=-|Q|$ für $Q \leq 0$ hat. Unsere Idee vorzeichenwechselnde $Q$ zuzulassen ist wie folgt: Zuerst schreiben wir $Q=Q_{+}-Q_{-}$ mit $Q_{ \pm}=|Q| \mathbb{1}_{A_{ \pm}}$, wobei $A_{+}:=\{Q>0\}$ und $A_{-}:=\{Q<0\}$ gesetzt seien. Dann führen wir die neue Gewichtsfunktion $Q_{\lambda}:=\lambda Q_{+}-Q_{-}$mit Parameter $\lambda>0$ ein. Wir betrachten dann für die Integralgleichung

$$
u=\mathbf{R}_{k}\left(Q_{\lambda}|u|^{p-2} u\right), \quad u \in L^{p}\left(\mathbb{R}^{N}\right)
$$

jeweils die Restriktionen $v:=\left.u\right|_{A_{+}}$und $w:=\left.u\right|_{A_{-}}$welches uns dann zum System

$$
\begin{aligned}
v & =\lambda \mathbb{1}_{A_{+}} \mathbf{R}_{k}\left[Q_{+}|v|^{p-2} v\right]-\mathbb{1}_{A_{+}} \mathbf{R}_{k}\left[Q_{-}|w|^{p-2} w\right], \\
w & =\lambda \mathbb{1}_{A_{-}} \mathbf{R}_{k}\left[Q_{+}|v|^{p-2} v\right]-\mathbb{1}_{A_{-}} \mathbf{R}_{k}\left[Q_{-}|w|^{p-2} w\right]
\end{aligned}
$$

führt. Mithilfe der dualen Transformationen

$$
\varphi:=\lambda Q_{+}^{1 / p^{\prime}}|v|^{p-2} v \in L^{p^{\prime}}\left(A_{+}\right), \quad \psi:=Q_{-}^{1 / p^{\prime}}|w|^{p-2} w \in L^{p^{\prime}}\left(A_{-}\right)
$$

erhalten wir aus obigem System das „duale“ System

$$
\begin{aligned}
\lambda^{1-p^{\prime}}|\varphi|^{p^{\prime}-2} \varphi & =Q_{+}^{1 / p} \mathbf{R}_{k}\left(|Q|^{1 / p}(\varphi-\psi)\right)=\mathbb{1}_{A_{+}} \mathcal{K}_{k}(\varphi-\psi), \\
|\psi|^{p^{\prime}-2} \psi & =Q_{-}^{1 / p} \mathbf{R}_{k}\left(|Q|^{1 / p}(\varphi-\psi)\right)=\mathbb{1}_{A_{-}} \mathcal{K}_{k}(\varphi-\psi)
\end{aligned}
$$


mit dem Birman-Schwinger Operator $\mathcal{K}_{k}: L^{p^{\prime}}\left(\mathbb{R}^{N}\right) \rightarrow L^{p}\left(\mathbb{R}^{N}\right)$ gegeben durch

$$
\mathcal{K}_{k}(f)=|Q|^{1 / p} \mathbf{R}_{k}\left(|Q|^{1 / p} f\right) .
$$

Dieses System trägt nun für $p \in\left[2_{*}, 2^{*}\right]$ eine variationelle Struktur mit dem dazugehörigen Funktional $J_{\lambda}: L^{p^{\prime}}\left(A_{+}\right) \times L^{p^{\prime}}\left(A_{-}\right) \rightarrow \mathbb{R}$ gegeben durch

$$
J_{\lambda}(\varphi, \psi):=\frac{\lambda^{1-p^{\prime}}}{p^{\prime}}\|\varphi\|_{p^{\prime}}^{p^{\prime}}-\frac{1}{p^{\prime}}\|\psi\|_{p^{\prime}}^{p^{\prime}}-\frac{1}{2} \int_{\mathbb{R}^{N}}(\varphi-\psi) \mathcal{K}_{k}(\varphi-\psi) d x .
$$

Das Funktional $J_{\lambda}$ ist von der Klasse $\mathcal{C}^{1}$ und kritische Punkte entsprechen Lösungen von

$$
-\Delta u-k^{2} u=Q_{\lambda}(x)|u|^{p-2} u \quad \text { in } \mathbb{R}^{N} .
$$

Anders als im definiten Fall besitzt $J_{\lambda}$ keine Mountain-Pass Geometrie und der Nachweis der Beschränktheit von Palais-Smale (oder auch Cerami) Folgen erweist sich als schwieriges Problem.

Wir leiten die Existenz kritischer Punkte von $J_{\lambda}$ in zwei Schritten her. Im ersten Schritt führen wir eine Sattelpunktsreduktion bezüglich der $\psi$-Variable durch. Genauer zeigen wir, dass falls $\mathcal{K}_{k}$ kompakt ist und $\int_{\mathbb{R}^{N}} \psi \mathcal{K}_{k}(\psi) d x \geq 0$ für alle $\psi \in L^{p^{\prime}}\left(A_{-}\right)$gilt, die Zuordnung $\psi \mapsto J_{\lambda}(\varphi, \psi)$ konkav und nach oben beschränkt ist. Damit gelingt es uns zu zeigen, dass für jedes $\varphi \in L^{p^{\prime}}\left(A_{+}\right)$genau ein $\psi^{*} \in L^{p^{\prime}}\left(A_{-}\right)$existiert mit

$$
J_{\lambda}\left(\varphi, \psi^{*}\right)=\max _{\psi \in L^{p^{\prime}}\left(A_{-}\right)} J_{\lambda}(\varphi, \psi):=\tilde{J}_{\lambda}(\varphi) .
$$

Dies gilt für jedes $\lambda>0$ und der gefundene Maximierer ist unabhängig von $\lambda$.

Im zweiten Schritt untersuchen wir das reduzierte Funktional $\tilde{J}_{\lambda}: L^{p^{\prime}}\left(A_{+}\right) \rightarrow \mathbb{R}$ auf kritische Punkte. Dieses besitzt für Werte $\lambda>\lambda_{0}$ eine Mountain-Pass Struktur, wobei $\lambda_{0}:=\left(2 \beta \alpha^{-1}\right)^{p}$ durch

$$
\alpha:=\max _{\substack{\|\varphi\|_{p^{\prime}=1}, \operatorname{supp}(\varphi) \subset A_{+}}} \int_{\mathbb{R}^{N}} \varphi \mathcal{K}_{k} \varphi d x, \quad \beta:=\max _{\substack{\|\varphi\|_{p^{\prime}=\|\psi\|_{p^{\prime}}=1,} \\ \operatorname{supp}(\varphi) \subset A_{+}, \operatorname{supp}(\psi) \subset A_{-} \mathbb{R}^{N}}} \int_{\mathcal{K}_{k}} \psi d x
$$

gegeben ist. Ist $\mathcal{K}_{k}$ kompakt, so werden beide Werte angenommen und wir haben $\beta \geq 0$. Zudem gilt $\alpha>0$, falls $\left|A_{+}\right|>0$, mithilfe der selben Idee wie im Beweis von [31, Lemma 4.2 (ii)]. Da $\tilde{J}_{\lambda}$ eine abstrakte Monotoniebeziehung in Bezug auf den Parameter $\lambda$ erfüllt, können wir [39. Theorem 2.1] anwenden, um die Existenz beschränkter Palais-Smale Folgen zu erhalten. Dieses Theorem erlaubt dies allerdings nur für fast alle $\lambda>\lambda_{0}$, wie für Monotonieaussagen dieser Art üblich. Unser Hauptresultat lautet wie folgt.

Theorem 1.45. Sei $N \geq 3$ und $p \in\left[2_{*}, 2^{*}\right)$ und sei $Q \in L^{\infty}\left(\mathbb{R}^{N}\right)$ mit $Q_{+} \not \equiv 0$. Zudem sei angenommen, dass

$$
\mathcal{K}_{k}: L^{p^{\prime}}\left(\mathbb{R}^{N}\right) \rightarrow L^{p}\left(\mathbb{R}^{N}\right) \text { ist kompakt und } \int_{\mathbb{R}^{N}} \psi \mathcal{K}_{k} \psi d x \geq 0, \quad \text { für alle } \psi \in L^{p^{\prime}}\left(A_{-}\right) .
$$


Dann gibt es für fast alle $\lambda>\lambda_{0}:=\left(2 \beta \alpha^{-1}\right)^{p}$ eine nichttriviale gebundene Zustandslösung von 1.92 .

Wie bereits gesehen, ist $\mathcal{K}_{k}$ bereits dann kompakt, falls $Q$ im unendlichen verschwindet. Die Nicht-Negativitätsbeziehung in 1.94 kann mithilfe von [15, Corollary 5.4] beispielsweise realisiert werden, falls $A_{-} \subset \mathbb{R}^{N}$ einen kleinen Durchmesser hat, nämlich falls $\operatorname{diam}\left(A_{-}\right) \leq$ $k^{-1} y_{\frac{N-2}{2}}$ gilt, wobei $y_{\frac{N-2}{2}}$ die erste positive Nullstelle der Bessel-Funktion $Y_{\frac{N-2}{2}}$ bezeichne.

Korollar 1.46. Sei $p \in\left[\frac{2(N+1)}{N-1}, \frac{2 N}{N-2}\right)$ und $Q \in L^{\infty}\left(\mathbb{R}^{N}\right), Q^{+} \not \equiv 0$. Zudem sei angenommen, dass

$$
\lim _{R \rightarrow \infty} \operatorname{esssup}_{|x| \geq R}|Q|=0 \quad \text { und } \quad \operatorname{diam}\left(A_{-}\right) \leq k^{-1} y_{\frac{N-2}{2}} .
$$

Dann existiert für fast alle $\lambda>\lambda_{0}:=\left(2 \beta \alpha^{-1}\right)^{p}$ eine nichttriviale gebundene Zustandslösung von (1.92). 


\section{Danksagungen}

Meinem Betreuer Herrn Prof. Dr. Tobias Weth möchte ich an dieser Stelle besonders herzlich danken. Sie haben mir durch Ihre stets aufmunternden Worte, Ihre eigene Begeisterung für das Thema, Ihre außerordentliche fachliche Expertise sowie Professionalität, Ihre Erfahrung und Gelassenheit und vor allem durch Ihre Geduld sowie Ihr Feingefühl in ganz besonderem Maße nicht nur in meinem wissenschaftlichen Werdegang unterstützt. Die Zeit bei Ihnen als Student und Doktorand war voller lehrreicher Erfahrungen und ich bin froh, dass Sie mich in meinem wissenschaftlichen Werdegang so umfassend unterstützt haben. Gerade in schwierigen Zeiten haben Sie stets zu mir gestanden und dafür bin ich Ihnen von ganzem Herzen dankbar. Ich bin froh und stolz, meine Ausbildung bei Ihnen absolviert zu haben. Danke!

Zudem gilt mein Dank Gilles Evéquoz. Schon als Student war Deine Tür immer für mich offen und Du hast stets ein Ohr für meine Fragen gehabt. Deine geduldige und clevere Art hat einen ganz großen Beitrag zu meinem Studium und später auch zur Promotion beigetragen und ich bin Dir für unsere gemeinsame Zeit sehr dankbar.

Des Weiteren Danke ich ganz herzlich Herrn Prof. Dr. Rupert L. Frank für unsere kurze, aber für mich erhellende fachliche Diskussion und natürlich Ihre Bereitschaft die vorliegende Dissertation zu begutachten. Ebenso Herrn von Harrach sowie Herrn Kersting für das Interesse am Thema sowie die Bereitschaft in der Prüfungskommission mitzuwirken.

Zudem möchte mich bei Rainer Mandel bedanken. Durch Deine Einladung nach Karlsruhe hast Du unseren wissenschaftlichen Austausch angeregt, worauf unsere gemeinsame Arbeit mit Dominic entstanden ist. Die vielen gemeinsamen Diskussionen, unser reger Austausch und Deine immer motivierende Art hat mir gerade im letzten Jahr enorm geholfen. Danke!

Mein ganz besonderer Dank gilt Dir, Jessica. Du hast mir immer stets zur Seite gestanden, mich bedingungslos unterstützt, mich aufgemuntert, mich abgelenkt und mir immer dabei geholfen nach vorne zu schauen. Du hast einen ganz besonderen Beitrag geleistet und meinen Dank hierfür kann ich kaum in Worte fassen.

Zudem gilt mein großer Dank meinen Eltern, Isa und Semra. Es ist schön, Euch stolz zu sehen und ich bin Euch für Eure unermüdliche Unterstützung sehr dankbar.

Zudem danke ich Euch Geli, Marcus, Mara und Shila. Nicht nur für den nötigen Rat, die Motivation und manchmal auch die Ablenkung, sondern ganz besonders dafür, dass Ihr Euch am finalen Tag so ins Zeug gelegt habt, um mit mir zu feiern. Das hat diesen Tag für mich ganz besonders gemacht und genau so möchte ich ihn in Erinnerung behalten. Danke! 


\section{CHAPTER 2}

\section{Dual ground state solutions for the critical nonlinear Helmholtz equation}

In this chapter we will present the existence of real-valued solutions to 2.1) in the Sobolevcritical case $p=2^{*}$. The presentation is in the very same form as the publication [Y1], which resulted from a collaboration with G. Evéquoz. Only the acknowledgement is excluded. The notation may slightly differ from the notation in Chapter 1.

\section{Introduction}

In this paper, we focus our attention on the existence of nontrivial real-valued solutions of the critical nonlinear Helmholtz equation

$$
-\Delta u-k^{2} u=Q(x)|u|^{p-2} u, \quad u \in W^{2, p}\left(\mathbb{R}^{N}\right)
$$

for $N \geq 3, k \neq 0$, and where $Q \in L^{\infty}\left(\mathbb{R}^{N}\right) \backslash\{0\}$ is a nonnegative weight function and $p=2^{*}:=\frac{2 N}{N-2}$ is the critical Sobolev exponent. Recently [31, the existence of solutions to (2.1) has been proven for all $p$ in the noncritical interval $\left(\frac{2(N+1)}{N-1}, \frac{2 N}{N-2}\right)$. A direct variational approach leads to some difficulties. Indeed, by classical results of Rellich [56] and Kato [40], solutions of the Helmholtz equation decay at most like

$$
u(x)=O\left(|x|^{-\frac{N-1}{2}}\right), \quad \text { as }|x| \rightarrow \infty .
$$

Therefore, solutions of 2.1 can only be expected to lie in $L^{p}\left(\mathbb{R}^{N}\right)$ or $W^{2, p}\left(\mathbb{R}^{N}\right)$ for $p>\frac{2 N}{N-1}$. In particular, $H^{1}$-solutions will not exist in general, as would be required when using the natural energy functional associated with (2.1).

The authors of [31] considered instead the integral equation

$$
u=\mathbf{R}_{k}\left(Q|u|^{p-2} u\right), \quad u \in L^{p}\left(\mathbb{R}^{N}\right),
$$

where $\mathbf{R}_{k}$ denotes the real part of the resolvent operator $\mathcal{R}_{k}: f \mapsto \Phi_{k} * f$ of $-\Delta-k^{2}$. Here, $\Phi_{k}$ is the (complex valued) radial outgoing fundamental solution of the Helmholtz equation, i.e, the convolution $\Phi_{k} * f$ solves the inhomogeneous Helmholtz equation $-\Delta u-k^{2} u=f$ and satisfies the Sommerfeld outgoing radiation condition

$$
\partial_{r} u(x)-\mathrm{i} k u(x)=o\left(|x|^{\frac{1-N}{2}}\right), \quad \text { as }|x| \rightarrow \infty .
$$


For $p \in\left[\frac{2(N+1)}{N-1}, \frac{2 N}{N-2}\right]$, solutions of the integral equation (2.2) by [31, lemma 4.3] belong to $W^{2, q}\left(\mathbb{R}^{N}\right)$ with $p \leq q<\infty$, so that, by Sobolev embeddings $u$ is indeed a strong solution of (2.1).

This dual variational approach, based on the dual energy functional $J_{Q, p}$ given by

$$
J_{Q, p}(v)=\frac{1}{p^{\prime}} \int_{\mathbb{R}^{N}}|v|^{p^{\prime}} \mathrm{d} x-\frac{1}{2} \int_{\mathbb{R}^{N}} v \mathbf{A}_{Q, p} v \mathrm{~d} x, \quad v \in L^{p^{\prime}}\left(\mathbb{R}^{N}\right),
$$

where $p^{\prime}=\frac{p}{p-1}$ and $\mathbf{A}_{Q, p} v=Q^{\frac{1}{p}} \mathbf{R}_{k}\left(Q^{\frac{1}{p}} v\right)$, admits a better behaved structure. The functional $J_{Q, p}$ is of class $C^{1}$ and has the mountain pass geometry. Therefore the properties of $Q$ determine whether it satisfies the Palais-Smale condition and this in turn is linked in an essential way to compactness properties of the Birman-Schwinger type operator $\mathbf{A}_{Q, p}$. For noncritical $p$, the operator $\mathbf{A}_{Q, p}$ is compact if $Q$ vanishes at infinity, and then $J_{Q, p}$ satisfies the Palais-Smale condition. When $Q$ is periodic, this is not the case anymore, but $\mathbf{A}_{Q, p}$ still has some local compactness. In combination with a crucial nonvanishing property [31, theorem 3.1] of the quadratic form associated with $\mathbf{R}_{k}$, a nontrivial critical point can then be obtained as weak limit after translation of a Palais-Smale sequence at the mountain pass level. The problem 2.1) becomes more delicate in the critical case $p=2^{*}$. Applying to the differential equation (2.1) the rescalings

$$
u \mapsto u_{r, x_{0}}, \quad \text { where } \quad u_{r, x_{0}}(x)=r^{\frac{N-2}{2}} u\left(r\left(x-x_{0}\right)\right),
$$

the linear term vanishes as $r \rightarrow \infty$ and, since the limit problem

$$
-\Delta u=Q\left(x_{0}\right)|u|^{2^{*}-2} u \quad \text { in } \mathbb{R}^{N}
$$

possesses nontrivial solutions, the local compactness of $\mathbf{A}_{Q}:=\mathbf{A}_{Q, 2^{*}}$ is lost. In the case where $Q$ vanishes at infinity, the functional $J_{Q}:=J_{Q, 2^{*}}$ therefore does not satisfy the Palais-Smale condition at every level. Indeed, if $u$ is a nontrivial solution of 2.4 for some $x_{0} \in \mathbb{R}^{N}$, consider the function $v$ given by

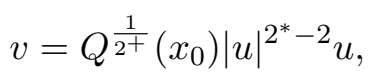

where $2^{+}=\frac{2 N}{N+2}$ is the conjugate exponent to $2^{*}$. Then, $v$ satisfies the dual equation

$$
|v|^{2^{+}-2} v=Q^{\frac{1}{2^{*}}}\left(x_{0}\right) \mathbf{R}_{0}\left(Q^{\frac{1}{2^{*}}}\left(x_{0}\right) v\right),
$$

where $\mathbf{R}_{0}=(-\Delta)^{-1}$. The sequence $\left(v_{n}\right)_{n}$ defined from $v$ via the dual rescalings $v_{n}(x):=$ $n^{\frac{N+2}{2}} v\left(n\left(x-x_{0}\right)\right)$ is then a Palais-Smale sequence for the dual functional $J_{Q}$ at level $c=$ $\frac{1}{N}\|v\|_{2^{+}}^{2^{+}}=\frac{1}{N}\|\nabla u\|_{2}^{2}$, and this sequence has no converging subsequence in $L^{2^{+}}\left(\mathbb{R}^{N}\right)$.

In analogy to the study of the critical problem 2.1 on a bounded domain, starting with the celebrated work of Brézis and Nirenberg [10], we shall try to recover some kind of compactness by comparing the mountain pass level $L_{Q}$ of the functional $J_{Q}$ with the least energy level 
$L_{Q}^{*}$ among all possible limiting problems 2.4 with $x_{0} \in \mathbb{R}^{N}$. From the duality between the Sobolev and the Hardy-Littlewood-Sobolev inequalities, it follows that

$$
L_{Q}^{*}=\frac{S^{\frac{N}{2}}}{N\|Q\|_{\infty}^{\frac{N-2}{2}}},
$$

where $S$ denotes the optimal constant in the Sobolev inequality (see $\S 3.2$ for more details).

The general strategy consists roughly in two steps:

(I) show that at every level $0<\beta<L_{Q}^{*}$, the Palais-Smale condition is satisfied, and

(II) establish the strict inequality $L_{Q}<L_{Q}^{*}$.

Ambrosetti and Struwe [5] confirmed that, for the Dirichlet problem on a bounded domain, this scheme is also adapted to the dual variational framework. However, whereas the authors in [5] reduce the proof of the Palais-Smale condition for the dual functional to the proof of the same property for the direct functional, we do not have for the problem (2.1) on $\mathbb{R}^{N}$ a direct functional at hand. In our approach towards the above steps (I) and (II), we choose instead to work directly with the resolvent operators for the original and the limit problems, via the corresponding fundamental solutions. More precisely, we start by deriving accurate upper and lower bounds on the difference of these fundamental solutions (lemma 2.2). This involves a detailed study of Bessel functions for small arguments. Based on these estimates, we then prove a new local compactness property for the difference operator $\mathbf{R}_{k}-\mathbf{R}_{0}$, where $\mathbf{R}_{0}=(-\Delta)^{-1}$ (see proposition 2.5). In addition, we show that $\mathbf{R}_{k}$ remains locally compact in subcritical Lebesgue spaces. Combining these properties, the step (I) can be completed in the case where $Q$ vanishes at infinity.

The next step is to prove the strict inequality $L_{Q}<L_{Q}^{*}$. There, the lower bound on the difference of the fundamental solutions plays a key role. Indeed, it implies that in dimension $N \geq 4$ the quadratic form of the operator $\mathbf{R}_{k}-\mathbf{R}_{0}$ is positive for positive functions supported in sets of small diameter. For such functions, the energy of $J_{Q}$ can thus be made smaller than that of the dual functional associated to 2.4. Since we are working with a nonconstant $Q$, an additional requirement (see (Q2) in theorem 2.1 below) is needed to complete the argument. The condition that we impose controls the way in which $Q$ approaches its maximum value $\|Q\|_{\infty}$. The same condition also appears in several related critical problems, and it seems to go back to the work of Escobar [25]. Let us mention that Egnell [24] provided examples of critical problems on bounded domains for which this assumption is necessary. More recently, this condition was also used in a paper by Chabrowski and Szulkin [14], on a strongly indefinite critical nonlinear Schrödinger equation on $\mathbb{R}^{N}$ with periodic coefficients. There, the authors work in a direct variational framework and use generalized linking arguments to show the existence of a Palais-Smale sequence at some level. The condition (Q2) is used to prove that this level lies strictly below $L_{Q}^{*}$. A nontrivial critical point is then obtained with the help of Lions' local compactness lemma [47,, 48] (see also [70, lemma 1.21]). Our approach to treat 
periodic, and more generally asymptotically periodic functions $Q$, is inspired by [14, although our arguments differ significantly. Working within the dual framework, we can simply use the mountain pass theorem without Palais-Smale condition, but we need to show that the nonvanishing property for $\mathbf{R}_{k}$, proven in [31, theorem 3.1] for noncritical exponents, continues to hold in the critical case $p=2^{*}$.

As already pointed out by Brézis and Nirenberg [10], there is a strong contrast between the dimensions $N=3$ and $N \geq 4$, for problems with the critical exponent. In the present case, the estimates on the difference of the fundamental solutions have the opposite sign for $N=3$, so that $\mathbf{R}_{k}-\mathbf{R}_{0}$ acts negatively on positive functions. This does not permit to verify Step (II) above, and we show that in fact $L_{Q}=L_{Q}^{*}$ holds for any bounded $Q \geq 0$ in this case. Moreover, we find that the mountain pass level $L_{Q}$ is not achieved.

As indicated in 31], every nontrivial critical point $v \in L^{2^{+}}\left(\mathbb{R}^{N}\right)$ of $J_{Q}$ is related, via the transformation

$$
u=\mathbf{R}_{k}\left(Q^{\frac{1}{2^{*}}} v\right)
$$

to a nontrivial strong solution $u \in W^{2,2^{*}}\left(\mathbb{R}^{N}\right)$ of (2.1) (see $\S 3.1$ for more details). The solutions we obtain in the present paper have the distinctive property that the corresponding critical point of $J_{Q}$ has minimal energy among all nontrivial critical points. Following the terminology introduced in a recent paper [28, we call such solutions dual ground states of (2.1) (cf. $\S 3$ for the precise definition). The main result in the present paper is the following.

Theorem 2.1. Let $N \geq 3$ and consider $Q \in L^{\infty}\left(\mathbb{R}^{N}\right) \backslash\{0\}$ such that $Q \geq 0$ a.e. in $\mathbb{R}^{N}$.

(i) If $N \geq 4$ and $Q$ satisfies the following conditions,

(Q1) $Q=Q_{p e r}+Q_{0}$, where $Q_{p e r}, Q_{0} \geq 0$ are such that $Q_{p e r}$ is periodic and $Q_{0}(x) \rightarrow 0$ as $|x| \rightarrow \infty$;

(Q2) there exists $x_{0} \in \mathbb{R}^{N}$ with $Q\left(x_{0}\right)=\max _{\mathbb{R}^{N}} Q$ and, as $\left|x-x_{0}\right| \rightarrow 0$,

$$
Q\left(x_{0}\right)-Q(x)= \begin{cases}o\left(\left|x-x_{0}\right|^{2}\right), & \text { if } N \geq 5, \\ O\left(\left|x-x_{0}\right|^{2}\right), & \text { if } N=4,\end{cases}
$$

then, the problem (2.1) with $p=2^{*}$ has a dual ground state.

(ii) If $N=3$, no dual ground state exists for 2.1) with $p=2^{*}$.

Note that the assumption (Q1) also allows for the cases $Q=Q_{0}$ and $Q=Q_{\text {per }}$. Let us point out that, for a maximum point $x_{0}$ of $Q$, the condition $Q\left(x_{0}\right)-Q(x)=O\left(\left|x-x_{0}\right|^{2}\right)$ as $x \rightarrow x_{0}$ is satisfied as soon as $Q$ is twice differentiable at $x_{0}$. The assumption $Q\left(x_{0}\right)-Q(x)=o\left(\left|x-x_{0}\right|^{2}\right)$ as $x \rightarrow x_{0}$ is more restrictive and requires some additional flatness of $Q$ at $x_{0}$ (cf. [24]). For small $k>0$, the condition (Q2) seems to be sharp. However, using a scaling argument, we can slightly weaken this assumption in dimensions $N \geq 5$ in the following sense: If $Q$ is given and $x_{0}$ is some maximum point of $Q$ for which $Q\left(x_{0}\right)-Q(x)=O\left(\left|x-x_{0}\right|^{2}\right)$ as $x \rightarrow x_{0}$. Then, if 
(Q1) is satisfied, there is some $k_{0}>0$ such that for all $k \geq k_{0}$ the equation (2.1) with $p=2^{*}$ has a dual ground state. Concerning the existence of multiple solutions, the method developed recently (see [28, Theorem 4.1]) for the high frequency limit $k \rightarrow \infty$ can be combined with the results of the present paper to relate the number of dual bound states (i.e., solutions of (2.1) associated to critical points of the dual functional) to the topology of the set $M$ of maximum points of $Q$. More precisely, for every given continuous $Q$ vanishing at infinity and satisfying the condition $Q\left(x_{0}\right)-Q(x)=O\left(\left|x-x_{0}\right|^{2}\right)$ as $x \rightarrow x_{0}$ for some of its maximum points $x_{0}$ there is $k^{*}>0$ such that for all $k \geq k^{*}$ the problem (2.1) has at least $\operatorname{cat}_{M_{\delta}}(M)$ dual bound states, where $M_{\delta}$ is some neighbourhood of $M$ and cat denotes the Ljusternik-Schnirelman category. Previous results on the critical equation 2.1 had been obtained in the radial case in [30] and very recently in [52], where a broad class of nonlinearities is considered. Up to our knowledge, theorem 2.1 is the first result concerning solutions of the nonlinear Helmholtz equation with critical nonlinearity and nonradial $Q$. Let us also mention that the lower critical case $p=\frac{2(N+1)}{N-1}$ is still open. There, we expect completely different phenomena than for $p=2^{*}$. A suitable method therefore needs to be found and we will address this issue in a forthcoming paper.

We shall now briefly describe the structure of the paper. In $\S 2$, we study the Helmholtz resolvent operator in the Lebesgue space $L^{2+}\left(\mathbb{R}^{N}\right)$. Recalling first the construction of the fundamental solution of the Helmholtz equation and its asymptotic properties, we derive in lemma 2.2 new upper and lower bounds on the difference of the latter and the fundamental solution of Laplace's equation, for small arguments. The proof consists in the precise estimation of Bessel functions and their derivatives, and the result is of crucial importance for the whole paper. As a first application, we prove in proposition 2.5 that the difference

$$
\mathbf{R}_{k}-\mathbf{R}_{0}: L^{2^{+}}\left(\mathbb{R}^{N}\right) \quad \rightarrow \quad L_{\mathrm{loc}}^{2^{*}}\left(\mathbb{R}^{N}\right)
$$

where $\mathbf{R}_{0}$ denotes the Laplace resolvent operator, is compact. There, we start by decomposing the fundamental solution of the Helmholtz equation in a similar way as in [31 and then apply the upper bounds obtained in lemma 2.2. Another essential property of the Helmholtz resolvent, the nonvanishing property, is established in the case $p=2^{*}$ in theorem 2.6. Its proof relies on improvements of previous results from [31] by means of the Hardy-Littlewood-Sobolev inequality. After this study of the Helmholtz resolvent, we turn in $\S 3$ to the existence of dual ground states of 2.1 with $p=2^{*}$. We start by recalling the dual variational framework set up in [31] and the characterization of the dual mountain pass level $L_{Q}$. Using the compactness properties of $\mathbf{R}_{k}$ and of $\mathbf{R}_{k}-\mathbf{R}_{0}$ established in $\S 2$, we then analyse the behaviour of PalaisSmale sequences for $J_{Q}$ at the level $L_{Q}$. Under the assumption $L_{Q}<L_{Q}^{*}$, we obtain in proposition 2.11 the existence of a nontrivial critical point for $J_{Q}$ in the case where $Q$ is asymptotically periodic. The nonvanishing property plays here a key role in handling the periodic part $Q_{\text {per }}$ of the coefficient $Q$. Section 3.3 is then devoted to estimating the dual 
mountain pass level $L_{Q}$ under the additional 'flatness condition' $(Q 2)$. There, we show that the positive lower bound on the difference of the fundamental solutions given by lemma 2.2 yields the strict inequality $L_{Q}<L_{Q}^{*}$, in the case $N \geq 4$. Combining the above results, we obtain in $\S 3.4$ the existence of dual ground states stated in theorem 2.1. The paper concludes with the 3 -dimensional case, in which we show that $L_{Q}=L_{Q}^{*}$ holds and, by a contradiction argument, we obtain the nonexistence of dual ground states for (2.1) with $p=2^{*}$ in this case.

We close this introduction by fixing some notation. Throughout the paper we denote by $B_{r}(x)$ the open ball in $\mathbb{R}^{N}$ with radius $r$ and centre at $x$. Moreover, we set $B_{r}=B_{r}(0)$. The constant

$\omega_{N}:=\frac{2 \pi^{\frac{N}{2}}}{N \Gamma\left(\frac{N}{2}\right)}$, where $\Gamma$ is the gamma function, represents the volume of the unit ball $B_{1}$. By $\mathbb{1}_{M}$ we shall denote the characteristic function of a measurable set $M \subset \mathbb{R}^{N}$. We write $\mathcal{S}\left(\mathbb{R}^{N}\right)$ for the space of Schwartz functions and $\mathcal{S}^{\prime}$ for its dual, i.e., the space of tempered distributions. Furthermore, we shall indifferently denote by $\widehat{f}$ or $\mathcal{F}(f)$ the Fourier transform of a function $f \in \mathcal{S}^{\prime}$. For $1 \leq s \leq \infty$, we abbreviate the norm in $L^{s}\left(\mathbb{R}^{N}\right)$ by $\|\cdot\|_{s}$.

\section{The Helmholtz-resolvent in the Sobolev-critical case}

2.1. Fundamental solutions. Without loss of generality and to simplify formulas, we consider the problem $(2.1)$ with $k=1$. The general case follows by rescaling the independent variable.

For $N \geq 3$, the radial outgoing fundamental solution of the Helmholtz equation $-\Delta u-u=\delta_{0}$ in $\mathbb{R}^{N}$ is given by

$$
\Phi(x):=\frac{\mathrm{i}}{4}(2 \pi|x|)^{\frac{2-N}{2}} H_{\frac{N-2}{2}}^{(1)}(|x|), \quad \text { for } x \in \mathbb{R}^{N} \backslash\{0\},
$$

where $H_{\frac{N-2}{2}}^{(1)}$ denotes the Hankel function of the first kind of order $\frac{N-2}{2}$. For a function $f \in$ $\mathcal{S}\left(\mathbb{R}^{N}\right)$ the convolution $u:=\Phi * f \in \mathcal{C}^{\infty}\left(\mathbb{R}^{N}\right)$ is a solution of the inhomogeneous Helmholtz equation $-\Delta u-u=f$ which satisfies the Sommerfeld outgoing radiation condition $\partial_{r} u(x)-$ $\mathrm{i} u(x)=o\left(|x|^{\frac{1-N}{2}}\right.$ ), as $|x| \rightarrow \infty$. Moreover, it is known (see [36]) that, in the sense of tempered distributions, the Fourier transform of $\Phi$ is given by

$$
\widehat{\Phi}(\xi)=(2 \pi)^{-\frac{N}{2}} \frac{1}{|\xi|^{2}-(1+\mathrm{i} 0)}:=(2 \pi)^{-\frac{N}{2}} \lim _{\varepsilon \rightarrow 0^{+}} \frac{1}{|\xi|^{2}-(1+\mathrm{i} \varepsilon)} .
$$

Since we shall be considering real-valued solutions of the Helmholtz equation in the sequel, we turn our attention to

$$
\Psi(x):=\operatorname{Re}(\Phi(x))=-\frac{1}{4}(2 \pi|x|)^{\frac{2-N}{2}} Y_{\frac{N-2}{2}}(|x|), \quad \text { for } x \in \mathbb{R}^{N} \backslash\{0\},
$$

where $Y_{\frac{N-2}{2}}$ denotes the Bessel function of the second kind of order $\frac{N-2}{2}$. $\Psi$ should be seen as the fundamental solution of the Helmholtz equation associated to real-valued standing waves. Let us recall some well-known facts concerning Bessel functions of the second kind: For nonnegative orders $\nu$ and positive arguments $t$, the asymptotic behaviour of $Y_{\nu}(t)$ is given by (see 
[44, Remark 5.16.2])

$$
\begin{array}{lll}
Y_{\nu}(t)=-\frac{2^{\nu} \Gamma(\nu)}{\pi t^{\nu}}(1+O(t)), & \text { as } t \rightarrow 0, \quad \text { if } \nu>0, \\
Y_{0}(t)=-\frac{2}{\pi} \ln \frac{2}{t}+O(1), & \text { as } t \rightarrow 0, & \\
Y_{\nu}(t)=-\sqrt{\frac{2}{\pi t}} \cos \left(t-\frac{(2 \nu-1) \pi}{4}\right)\left(1+O\left(t^{-1}\right)\right), & \text { as } t \rightarrow \infty, \quad \text { for all } \nu \geq 0 .
\end{array}
$$

As a consequence, we find that

$$
\Psi(x)= \begin{cases}\frac{1}{N(N-2) \omega_{N}}|x|^{2-N}(1+O(|x|)), & \text { as }|x| \rightarrow 0 \\ \frac{1}{2}(2 \pi|x|)^{\frac{1-N}{2}} \cos \left(|x|-\frac{(N-3) \pi}{4}\right)\left(1+O\left(|x|^{-1}\right)\right), & \text { as }|x| \rightarrow \infty\end{cases}
$$

Denoting by $y_{\nu}$ the first positive zero of $Y_{\nu}$ with $\nu \geq 0$, we deduce from the asymptotics (2.8) and (2.9), that $Y_{\nu}(t)<0$ for all $t \in\left(0, y_{\nu}\right)$ and therefore $\Psi(x)>0$ for all $|x|<y_{\nu}$.

Recalling that for $N \geq 3$ the fundamental solution $\Lambda$ of Laplace's equation in $\mathbb{R}^{N}$ is given by

$$
\Lambda(x)=\frac{1}{N(N-2) \omega_{N}}|x|^{2-N}, \quad \text { for } x \in \mathbb{R}^{N} \backslash\{0\},
$$

we see from 2.11 that $\Psi(x)$ behaves like $\Lambda(x)$ for small $|x|$. Our first result gives more precise estimates on the way $\Psi(x)$ approaches $\Lambda(x)$ as $|x| \rightarrow 0$. In particular, we observe a strong contrast between the dimension $N=3$ and the higher dimensions $N \geq 4$.

LEMma 2.2. Let $r>0$ be given such that $r<y_{\frac{N-4}{2}}$ if $N \geq 4$ and $r<\pi$ if $N=3$.

(i) There exist $\kappa_{1}, \kappa_{2}>0$ only depending on $r$ and $N$, such that for all $x \in B_{r}$,

$$
\left\{\begin{array}{l}
\kappa_{1}|x|^{4-N} \leq \Psi(x)-\Lambda(x) \leq \kappa_{2}|x|^{4-N}, \quad \text { if } N \geq 5 \\
\kappa_{1}|\ln | x|| \leq \Psi(x)-\Lambda(x) \leq \kappa_{2}|\ln | x||, \quad \text { if } N=4, \\
-\kappa_{1}|x| \quad \geq \Psi(x)-\Lambda(x) \geq-\kappa_{2}|x|, \quad \text { if } N=3 .
\end{array}\right.
$$

(ii) For every multiindex $\alpha \in \mathbb{N}_{0}^{N}$ with $|\alpha| \geq 1$, there exists $\kappa_{3}>0$ only depending on $|\alpha|$, $r$ and $N$, such that

$$
\left|\partial^{\alpha}(\Psi(x)-\Lambda(x))\right| \leq \kappa_{3}|x|^{4-N-|\alpha|}, \quad \text { for all } x \in B_{r}
$$

Proof. We start by considering for $\nu \geq 1$ the function $\eta_{\nu}:[0, \infty) \rightarrow \mathbb{R}$ given by

$$
\eta_{\nu}(t):=\left\{\begin{array}{ll}
-c_{\nu} t^{\nu} Y_{\nu}(t), & t>0, \\
1, & t=0,
\end{array} \quad \text { where } c_{\nu}=\frac{\pi}{2^{\nu} \Gamma(\nu)} .\right.
$$


Remark that $\eta_{\nu}$ is continuous, as a consequence of 2.8 and since $Y_{\nu}$ is analytic on $(0, \infty)$. In addition, for $t>0$, the recursion formula $\frac{\mathrm{d}}{\mathrm{d} t}\left[t^{\nu} Y_{\nu}(t)\right]=t^{\nu} Y_{\nu-1}(t)$ (see [44, p.105]) gives

$$
\eta_{\nu}^{\prime}(t)=-c_{\nu} t^{\nu} Y_{\nu-1}(t)
$$

Hence, $\eta_{\nu}$ is strictly increasing in the interval $\left(0, y_{\nu-1}\right)$ and in particular $\eta_{\nu}>1$ in this interval. Moreover, using the asymptotic expansions for small arguments (2.8) and (2.9), we see that

$$
\begin{aligned}
\lim _{t \rightarrow 0^{+}} \frac{\eta_{\nu}^{\prime}(t)}{t} & =-c_{\nu} \lim _{t \rightarrow 0^{+}} t^{\nu-1} Y_{\nu-1}(t)=\frac{1}{2(\nu-1)}, \quad \text { if } \nu>1, \\
\text { and } \quad \lim _{t \rightarrow 0^{+}} \frac{\eta_{1}^{\prime}(t)}{t|\ln t|} & =-\frac{\pi}{2} \lim _{t \rightarrow 0^{+}} \frac{Y_{0}(t)}{-\ln t}=1 .
\end{aligned}
$$

Therefore, given $0<r<y_{\nu-1}$ and since $y_{0}<1$, there exist constants $\kappa_{1}^{\prime}=\kappa_{1}^{\prime}(\nu, r)$ and $\kappa_{2}^{\prime}(\nu, r)$ such that

$$
\begin{gathered}
\frac{\eta_{\nu}^{\prime}(t)}{t} \geq 2 \kappa_{1}^{\prime}, \quad \text { if } \nu>1, \quad \text { and } \quad \frac{\eta_{1}^{\prime}(t)}{t|\ln t|} \geq 2 \kappa_{1}^{\prime}, \quad \text { for all } 0<t<r, \\
\left|\frac{\eta_{\nu}^{\prime}(t)}{t}\right| \leq 2 \kappa_{2}^{\prime}, \quad \text { if } \nu>1, \quad \text { and } \quad\left|\frac{\eta_{1}^{\prime}(t)}{t \ln t}\right| \leq 2 \kappa_{2}^{\prime}, \quad \text { for all } 0<t<r .
\end{gathered}
$$

Writing

$$
\frac{\eta_{\nu}(t)-1}{t^{2}}=\int_{0}^{1} s \frac{\eta_{\nu}^{\prime}(s t)}{s t} \mathrm{~d} s \quad \text { and } \quad \frac{\eta_{1}(t)-1}{t^{2}|\ln t|}=\int_{0}^{1} s\left|\frac{\ln (s t)}{\ln t}\right| \frac{\eta_{1}^{\prime}(s t)}{s t|\ln (s t)|} \mathrm{d} s,
$$

we obtain the bounds

$$
\begin{array}{cll}
\eta_{\nu}(t)-1 \geq \kappa_{1}^{\prime} t^{2}, & \text { if } \nu>1, \quad \text { and } & \eta_{1}(t)-1 \geq \kappa_{1}^{\prime} t^{2}|\ln t|, \quad \text { for all } 0<t<r, \\
\left|\eta_{\nu}(t)-1\right| \leq \kappa_{2}^{\prime} t^{2}, & \text { if } \nu>1, \quad \text { and } \quad & \left|\eta_{1}(t)-1\right| \leq \kappa_{2}^{\prime \prime} t^{2}|\ln t|, \quad \text { for all } 0<t<r,
\end{array}
$$

with some $\kappa_{2}^{\prime \prime}=\kappa_{2}^{\prime \prime}(r)>0$. The assertion (i) in case $N \geq 4$ follows from (2.14) and 2.15), since we have

$$
\Psi(x)-\Lambda(x)=\Lambda(x)\left(\eta_{\frac{N-2}{2}}(|x|)-1\right)=\frac{1}{N(N-2) \omega_{N}}|x|^{2-N}\left(\eta_{\frac{N-2}{2}}(|x|)-1\right) .
$$

In the case $N=3$, we have

$$
\Psi(x)-\Lambda(x)=\frac{1}{4 \pi|x|}(\cos |x|-1)
$$

Remark that

$$
\frac{\cos t-1}{t^{2}}=-\int_{0}^{1} s \frac{\sin (s t)}{s t} \mathrm{~d} s, \quad t \mapsto \frac{\sin t}{t} \text { is decreasing in }[0, \pi], \quad \lim _{t \rightarrow 0} \frac{\sin t}{t}=1,
$$

and $\left|\frac{\sin t}{t}\right| \leq 1$ for all $t>0$. We thus conclude that for given $0<r_{0}<\pi$ there is a constant $\kappa_{1}=\kappa_{1}\left(r_{0}\right)>0$ such that $\frac{\cos t-1}{t^{2}} \leq-\kappa_{1}, \quad$ for all $0<t<r_{0}, \quad$ and $\quad \frac{\cos t-1}{t^{2}} \geq-\frac{1}{2}, \quad$ for all $t>0$. 
Plugging these estimates in 2.16 yields the assertion (i) for $N=3$ with $\kappa_{2}=\frac{1}{8 \pi}$.

To prove the assertion (ii), we notice that for $\alpha \in \mathbb{N}_{0}^{N}$ and $k=|\alpha|$, an induction argument based on the recursion formula $\frac{\mathrm{d}}{\mathrm{d} t}\left[t^{-\nu} Y_{\nu}(t)\right]=-t^{-\nu} Y_{\nu+1}(t)$ (see [44, p.105]) gives

$$
\partial^{\alpha}(\Psi(x)-\Lambda(x))=\sum_{\ell=0}^{\left\lfloor\frac{k}{2}\right\rfloor} f_{k-\ell}(|x|) P_{k-2 \ell}(x),
$$

where for $m \in \mathbb{N}_{0}, P_{m}(x)$ is a homogeneous polynomial of degree $m$ and where

$$
f_{m}(t)=(-1)^{m} \frac{2^{m} \Gamma\left(\frac{N-2}{2}+m\right)}{\Gamma\left(\frac{N-2}{2}\right)} \frac{t^{2-N-2 m}}{N(N-2) \omega_{N}}\left[\eta_{\frac{N-2}{2}+m}(t)-1\right], \quad t>0 .
$$

As a consequence, given $r>0$, there is a constant $\gamma=\gamma(N, k, r)>0$ such that

$$
\left|\partial^{\alpha}(\Psi(x)-\Lambda(x))\right| \leq \gamma|x|^{2-N-k} \sum_{\ell=0}^{\left\lfloor\frac{k}{2}\right\rfloor}\left[\eta_{\frac{N-2}{2}+k-\ell}(|x|)-1\right], \quad \text { for all }|x|<r .
$$

Using (2.15) and remarking that $\frac{N-2}{2}+k-\ell>1$ for $k \geq 1$ and $0 \leq \ell \leq\left\lfloor\frac{k}{2}\right\rfloor$, we obtain the desired assertion and the lemma is proven.

2.2. Compactness properties. Here, and in the next section, we discuss the properties of the resolvent Helmholtz operator $\mathbf{R}:=\mathbf{R}_{1}$, given by the convolution $f \mapsto \Psi * f$ for $f \in \mathcal{S}\left(\mathbb{R}^{N}\right)$ where $\Psi$ is given in (2.7). Let us first remark, that as a consequence of an estimate of Kenig, Ruiz ans Sogge [43, Theorem 2.3], this mapping extends as a continuous linear operator

$$
\mathbf{R}: L^{2^{+}}\left(\mathbb{R}^{N}\right) \rightarrow L^{2^{*}}\left(\mathbb{R}^{N}\right) .
$$

In particular, there exists a constant $C_{0}>0$ only depending on $N$ such that

$$
\|\mathbf{R} v\|_{2^{*}} \leq C_{0}\|v\|_{2^{+}}, \quad \text { for all } v \in L^{2^{+}}\left(\mathbb{R}^{N}\right)
$$

Let us denote by

$$
\mathbf{R}_{0}: L^{2^{+}}\left(\mathbb{R}^{N}\right) \rightarrow L^{2^{*}}\left(\mathbb{R}^{N}\right)
$$

the linear operator given by the convolution with the fundamental solution of Laplace's equation

$$
\mathbf{R}_{0} v:=\Lambda * v, \quad v \in L^{2^{*}}\left(\mathbb{R}^{N}\right) .
$$

Notice that $\mathbf{R}_{0}$ is well defined and continuous, as a consequence of the weak Yound inequality [46, p.107].

REMARK 2.3. The results in this and the next sections are stated and proven for the real part $\mathbf{R}$ of the resolvent, but they remain valid for the full resolvent $\mathcal{R}: L^{2^{+}}\left(\mathbb{R}^{N}\right) \rightarrow L^{2^{*}}\left(\mathbb{R}^{N}\right)$ which is the extension of the convolution map $f \mapsto \Phi * f, f \in \mathcal{S}\left(\mathbb{R}^{N}, \mathbb{C}\right)$.

Lemma 2.4. For all $1 \leq t<2^{*}$ and all $r>0$ the operator $\mathbb{1}_{B_{r}} \mathbf{R}: L^{2^{+}}\left(\mathbb{R}^{N}\right) \rightarrow L^{t}\left(\mathbb{R}^{N}\right)$ is compact. 
Proof. By elliptic estimates (see [31, proposition A.1]), we can find for every $r>0$ a constant $D_{r}>0$ such that $\|\mathbf{R} v\|_{W^{2,2}{ }_{\left(B_{r}\right)}} \leq D_{r}\|v\|_{2^{+}}$for all $v \in L^{2^{+}}\left(\mathbb{R}^{N}\right)$. Since the embedding $W^{2,2^{+}}\left(B_{r}\right) \hookrightarrow L^{t}\left(B_{r}\right)$ is compact for all $1 \leq t<2^{*}$, and all $r>0$, we deduce that the operator $\mathbb{1}_{B_{r}} \mathbf{R}: L^{2^{+}}\left(\mathbb{R}^{N}\right) \rightarrow L^{t}\left(\mathbb{R}^{N}\right)$ is compact for all $1 \leq t<2^{*}$ and all $r>0$.

\section{Proposition 2.5.}

(i) The difference $\mathbf{R}-\mathbf{R}_{0}$ is a continuous linear mapping from $L^{2^{+}}\left(\mathbb{R}^{N}\right)$ into $W^{2,2^{*}}\left(\mathbb{R}^{N}\right)$

(ii) For all $r>0$, the operator $\mathbb{1}_{B_{r}}\left(\mathbf{R}-\mathbf{R}_{0}\right): L^{2^{+}}\left(\mathbb{R}^{N}\right) \rightarrow L^{2^{*}}\left(\mathbb{R}^{N}\right)$ is compact.

Proof. In the sequel, for $\mu \in \mathbb{R}, C$ and $C_{\mu}$ shall denote constants depending on $N$ and on $N, \mu$ respectively, but which may change from line to line.

To prove (i) we shall use a decomposition of $\Psi$, similar to the one introduced in [31, §3] for $\Phi$. We fix a radial $\psi \in \mathcal{S}\left(\mathbb{R}^{N}\right)$ such that $\widehat{\psi} \in \mathcal{C}_{c}^{\infty}\left(\mathbb{R}^{N}\right), 0 \leq \widehat{\psi} \leq 1, \widehat{\psi}(\xi)=1$ for ||$\xi|-1| \leq \frac{1}{6}$ and $\widehat{\Psi}(\xi)=0$ for ||$\xi|-1| \geq \frac{1}{4}$. Write $\Psi=\Psi_{1}+\Psi_{2}$ with

$$
\Psi_{1}:=(2 \pi)^{-\frac{N}{2}}(\Psi * \psi), \quad \Psi_{2}=\Psi-\Psi_{1} .
$$

Then, for every $f \in \mathcal{S}\left(\mathbb{R}^{N}\right)$ and $\alpha \in \mathbb{N}_{0}^{N}$, the properties of the convolution of Schwartz functions with a tempered distribution (see [57, theorem 7.19]) allow to write

$$
\left(\partial^{\alpha} \Psi_{1}\right) * f=(2 \pi)^{-\frac{N}{2}}\left[\Psi *\left(\partial^{\alpha} \psi\right)\right] * f=(2 \pi)^{-\frac{N}{2}} \Psi *\left[\left(\partial^{\alpha} \psi\right) * f\right],
$$

where $\partial^{\alpha} \psi \in \mathcal{S}\left(\mathbb{R}^{N}\right)$. Hence, from the resolvent estimate (1.9) and Young's inequality for the convolution, we obtain the estimate

$$
\left\|\left(\partial^{\alpha} \Psi_{1}\right) * f\right\|_{2^{*}}=(2 \pi)^{-\frac{N}{2}}\left\|\Psi *\left[\left(\partial^{\alpha} \psi\right) * f\right]\right\|_{2^{*}} \leq(2 \pi)^{-\frac{N}{2}} C_{0}\left\|\partial^{\alpha} \psi\right\|_{1}\|f\|_{2^{+}},
$$

for all $f \in \mathcal{S}\left(\mathbb{R}^{N}\right)$. As a consequence, the convolution $f \mapsto\left(\partial^{\alpha} \Psi_{1}\right) * f, f \in \mathcal{S}\left(\mathbb{R}^{N}\right)$, extends as a continuous map from $L^{2^{+}}\left(\mathbb{R}^{N}\right)$ into $L^{2^{*}}\left(\mathbb{R}^{N}\right)$ for every $\alpha \in \mathbb{N}_{0}^{N}$.

Turning to $\Psi_{2}$, we have by definition $\widehat{\Psi_{2}}=(1-\widehat{\psi}) \widehat{\Psi}$ and, since taking real parts in 3.30 yields

$$
\widehat{\Psi_{2}}(\xi)=(2 \pi)^{-\frac{N}{2}} \lim _{\varepsilon \rightarrow 0^{+}} \frac{|\xi|^{2}-1}{\left(|\xi|^{2}-1\right)^{2}+\varepsilon^{2}}(1-\widehat{\psi}(\xi))=(2 \pi)^{-\frac{N}{2}} \frac{1-\widehat{\psi}(\xi)}{|\xi|^{2}-1},
$$

we get $\widehat{\Psi_{2}} \in \mathcal{C}^{\infty}\left(\mathbb{R}^{N}\right)$ and $\widehat{\Psi_{2}}(\xi)=(2 \pi)^{-\frac{N}{2}}\left(|\xi|^{2}-1\right)^{-1}$ for $|\xi| \geq \frac{5}{4}$. This gives $\partial^{\beta} \widehat{\Psi_{2}} \in L^{1}\left(\mathbb{R}^{N}\right)$ for all $\beta \in \mathbb{N}_{0}^{N}$ such that $2+|\beta|>N$. Therefore, using standard differentiation properties of the Fourier transform, the fact that $\widehat{\Psi_{2}}$ (and so $\left.\Psi_{2}\right)$ is radial and that $\mathcal{F}(f)(\xi)=\mathcal{F}^{-1}(f)(-\xi)$, we obtain

$$
\left\||\cdot|{ }^{|\beta|} \Psi_{2}\right\|_{\infty}=\left\|\mathcal{F}\left(\partial^{\beta} \widehat{\Psi_{2}}\right)\right\|_{\infty} \leq\left\|\partial^{\beta} \widehat{\Psi_{2}}\right\|_{L^{1}\left(\mathbb{R}^{N}\right)} \leq C_{|\beta|}, \quad \text { for all }|\beta|>N-2
$$

Choosing $\beta \in \mathbb{N}_{0}^{N}$ with $|\beta|=N$, we obtain that

$$
\left|\Psi_{2}(x)\right| \leq C|x|^{-N}, \quad \text { for all } x \in \mathbb{R}^{N} .
$$


Using the same argument with $\partial^{\alpha} \Psi_{2}$ in place of $\Psi_{2}$, for every $\alpha \in \mathbb{N}_{0}^{N}$, we get

$$
\left|\partial^{\alpha} \Psi_{2}(x)\right| \leq C_{|\alpha|}|x|^{-N-|\alpha|}, \quad \text { for all } x \in \mathbb{R}^{N} \text { and all } \alpha \in \mathbb{N}_{0}^{N} \text {. }
$$

From lemma 2.2, we obtain estimates on $\partial^{\alpha}\left(\Psi_{2}-\Lambda\right)(x)$ for $|x|$ small. For large values of $|x|$, we use (2.20) and $\left|\partial^{\alpha} \Lambda(x)\right| \leq C_{|\alpha|}|x|^{2-N-|\alpha|}$, which follows easily from (2.12). Altogether, we get for $\alpha \in \mathbb{N}_{0}^{N}$ and $x \in \mathbb{R}^{N}$,

$$
\left|\partial^{\alpha}\left(\Psi_{2}-\Lambda\right)(x)\right| \leq \begin{cases}C_{|\alpha|} \min \left\{|x|^{4-N-|\alpha|},|x|^{2-N-|\alpha|}\right\}, & N=3, N \geq 5, \text { or } \\ & N=4 \text { and }|\alpha| \geq 1, \\ C \min \left\{1+|\ln | x||,|x|^{-2}\right\}, & N=4 \text { and }|\alpha|=0 .\end{cases}
$$

As a consequence, denoting by $L_{w}^{\frac{N}{N-2}}\left(\mathbb{R}^{N}\right)$ the weak- $L^{\frac{N}{N-2}}$ space (see e.g. [46, p.106]), we infer that

$$
\partial^{\alpha}\left(\Psi_{2}-\Lambda\right) \in L_{w}^{\frac{N}{N-2}}\left(\mathbb{R}^{N}\right), \quad \text { for all } \alpha \in \mathbb{N}_{0}^{N} \text { such that }|\alpha| \leq 2 .
$$

From the weak Young inequality, the convolution $f \mapsto \partial^{\alpha}\left(\Psi_{2}-\Lambda\right) * f, f \in \mathcal{S}\left(\mathbb{R}^{N}\right)$, extends as a continuous map from $L^{2^{+}}\left(\mathbb{R}^{N}\right)$ into $L^{2^{*}}\left(\mathbb{R}^{N}\right)$ for such $\alpha$. Summarizing and using the fact that

$$
\|(\Psi-\Lambda) * f\|_{W^{2,2^{*}}}^{2} \leq 2 \sum_{|\alpha| \leq 2}\left\|\partial^{\alpha}\left(\Psi_{2}-\Lambda\right) * f\right\|_{2^{*}}^{2}+2 \sum_{|\alpha| \leq 2}\left\|\left(\partial^{\alpha} \Psi_{1}\right) * f\right\|_{2^{*}}^{2},
$$

we obtain that the convolution $f \mapsto(\Psi-\Lambda) * f$ extends as a continuous map from $L^{2^{+}}\left(\mathbb{R}^{N}\right)$ into $W^{2,2^{*}}\left(\mathbb{R}^{N}\right)$. Therefore, the operator

$$
\mathbf{R}-\mathbf{R}_{0}: L^{2^{+}}\left(\mathbb{R}^{N}\right) \rightarrow W^{2,2^{*}}\left(\mathbb{R}^{N}\right)
$$

is continuous and (i) is proven.

By the Rellich-Kondrachov theorem, the embedding $W_{l o c}^{2,2^{*}}\left(\mathbb{R}^{N}\right) \hookrightarrow L_{l o c}^{t}\left(\mathbb{R}^{N}\right)$ is compact for all $1 \leq t<\frac{2 N}{(N-6)_{+}}$. Thus, we obtain the compactness of $\mathbb{1}_{B_{r}}\left(\mathbf{R}-\mathbf{R}_{0}\right): L^{2^{+}}\left(\mathbb{R}^{N}\right) \rightarrow L^{2^{*}}\left(\mathbb{R}^{N}\right)$ for all $r>0$, which proves (ii).

2.3. Nonvanishing property and related estimates. As a key ingredient for the upcoming existence results, we prove that the nonvanishing property of the quadratic form associated with the Helmholtz resolvent holds true in the space $L^{p^{\prime}}\left(\mathbb{R}^{N}\right)$ with $p=2^{*}$. This property has been proven in [31, theorem 3.1] in the noncritical range $\frac{2(N+1)}{N-1}<p<2^{*}$.

THEOREM 2.6. Consider a bounded sequence $\left(v_{n}\right)_{n} \subset L^{2^{+}}\left(\mathbb{R}^{N}\right)$ satisfying

$$
\limsup _{n \rightarrow \infty}\left|\int_{\mathbb{R}^{N}} v_{n} \mathbf{R} v_{n} \mathrm{~d} x\right|>0 .
$$


Then there exists $R>0, \zeta>0$ and a sequence $\left(x_{n}\right)_{n} \subset \mathbb{R}^{N}$ such that, up to a subsequence,

$$
\int_{B_{R}\left(x_{n}\right)}\left|v_{n}\right|^{2^{+}} \mathrm{d} x \geq \zeta, \quad \text { for all } n .
$$

Proof. Let us assume by contradiction that

$$
\lim _{n \rightarrow \infty}\left(\sup _{y \in \mathbb{R}^{N}} \int_{B_{\rho}(y)}\left|v_{n}\right|^{2^{+}} \mathrm{d} x\right)=0, \quad \text { for all } \rho>0 .
$$

Consider the decomposition $\Psi=\Psi_{1}+\Psi_{2}$ introduced in 2.18$)$, and denote by $\mathbf{R}_{1}: L^{2^{+}}\left(\mathbb{R}^{N}\right)$ $\rightarrow L^{2^{*}}\left(\mathbb{R}^{N}\right)$ the continuous extension of the convolution map $f \mapsto \Psi_{1} * f, f \in \mathcal{S}\left(\mathbb{R}^{N}\right)$. Since Lemma 3.4 in [31] holds for the critical exponent $p=2^{*}$, we obtain by density of $\mathcal{S}\left(\mathbb{R}^{N}\right)$ into $L^{2^{+}}\left(\mathbb{R}^{N}\right)$ that

$$
\int_{\mathbb{R}^{N}} v_{n} \mathbf{R}_{1} v_{n} \mathrm{~d} x \rightarrow 0, \quad \text { as } n \rightarrow \infty,
$$

taking real parts. Turning to $\Psi_{2}$, we note that the estimate 2.19) and the behaviour of $\Psi$ close to $x=0$ given by (2.11) yield the existence of some constant $C^{\prime}=C^{\prime}(N)>0$ such that

$$
\left|\Psi_{2}(x)\right| \leq C^{\prime} \min \left\{|x|^{2-N},|x|^{-N}\right\}, \quad \text { for all } x \neq 0 .
$$

Setting $M_{R}:=\mathbb{R}^{N} \backslash B_{R}$ for $R>1$, we deduce from 2.26 that

$$
\left\|\Psi_{2}\right\|_{L^{\frac{N}{N-2}}\left(M_{R}\right)} \leq C^{\prime}\left(\int_{|x| \geq R}|x|^{-\frac{N^{2}}{N-2}} d x\right)^{\frac{N-2}{N}} \rightarrow 0, \quad \text { as } R \rightarrow \infty .
$$

Hence, by Young's inequality,

$$
\sup _{n \in \mathbb{N}}\left|\int_{\mathbb{R}^{N}} v_{n}\left[\left(\mathbb{1}_{M_{R}} \Psi_{2}\right) * v_{n}\right] \mathrm{d} x\right| \leq\left\|\Psi_{2}\right\|_{L^{\frac{N}{N-2}}\left(M_{R}\right)} \sup _{n \in \mathbb{N}}\left\|v_{n}\right\|_{L^{2^{+}}\left(\mathbb{R}^{N}\right)}^{2} \rightarrow 0, \quad \text { as } R \rightarrow \infty .
$$

Consider a decomposition of $\mathbb{R}^{N}$ into disjoint $N$-cubes $\left\{Q_{\ell}\right\}_{\ell \in \mathbb{N}}$ of side length $R$, and let for each $\ell$ the $N$-cube $Q_{\ell}^{\prime}$ have the same centre as $Q_{\ell}$ but side length $3 R$. From the estimate (2.26) and the Hardy-Littlewood-Sobolev inequality [46, theorem 4.3], there is a constant $C^{\prime \prime}=C^{\prime \prime}(N)$ 
such that

$$
\begin{aligned}
\left|\int_{\mathbb{R}^{N}} v_{n}\left[\left(\mathbb{1}_{B_{R}} \Psi_{2}\right) * v_{n}\right] \mathrm{d} x\right| & \leq \sum_{\ell=1}^{\infty} \int_{Q_{\ell}}\left(\int_{|x-y|<R}\left|\Psi_{2}(x-y)\right|\left|v_{n}(x)\right|\left|v_{n}(y)\right| \mathrm{d} y\right) \mathrm{d} x \\
& \leq C^{\prime} \sum_{\ell=1}^{\infty} \int_{Q_{\ell}}\left(\int_{Q_{\ell}^{\prime}} \frac{\left|v_{n}(x)\right|\left|v_{n}(y)\right|}{|x-y|^{N-2}} \mathrm{~d} y\right) \mathrm{d} x \\
& \leq C^{\prime \prime} \sum_{\ell=1}^{\infty}\left(\int_{Q_{\ell}^{\prime}}\left|v_{n}(x)\right|^{2^{+}} \mathrm{d} x\right)^{\frac{N+2}{N}} \\
& \leq C^{\prime \prime}\left[\sup _{\ell \in \mathbb{N}} \int_{Q_{\ell}^{\prime}}\left|v_{n}(x)\right|^{2^{+}} \mathrm{d} x\right]^{\frac{2}{N}} \sum_{\ell=1}^{\infty} \int_{Q_{\ell}^{\prime}}\left|v_{n}(x)\right|^{2^{+}} \mathrm{d} x \\
& \leq C^{\prime \prime}\left[\sup _{y \in \mathbb{R}^{N}} \int_{B_{3 R \sqrt{N}}(y)}\left|v_{n}(x)\right|^{2^{+}} \mathrm{d} x\right]^{\frac{2}{N}} 3^{N}\left\|v_{n}\right\|_{2^{+}}^{2^{+}},
\end{aligned}
$$

for all $n$. Therefore, the boundedness of $\left(v_{n}\right)_{n}$ and the assumption 2.24 give

$$
\lim _{n \rightarrow \infty} \int_{\mathbb{R}^{N}} v_{n}\left[\left(\mathbb{1}_{B_{R}} \Psi_{2}\right) * v_{n}\right] \mathrm{d} x=0, \quad \text { for every } R>0 .
$$

Combining 2.25), 2.27) and 2.28), we obtain

$$
\int_{\mathbb{R}^{N}} v_{n} \mathbf{R} v_{n} \mathrm{~d} x=\int_{\mathbb{R}^{N}} v_{n} \mathbf{R}_{1} v_{n} \mathrm{~d} x+\int_{\mathbb{R}^{N}} v_{n}\left[\Psi_{2} * v_{n}\right] \mathrm{d} x \rightarrow 0, \quad \text { as } n \rightarrow \infty,
$$

contradicting the assumption 2.22. The theorem follows.

Let us recall a result obtained in [28, Lemma 2.4], on the bilinear form associated to the operator $\mathbf{R}$ for functions having disjoint support.

Lemma 2.7. [28, Lemma 2.4] Let $p>\frac{2(N+1)}{N-1}$. There exists a constant $D=D(N, p)>0$ such that for any $R>0, r \geq 1$ and $u, v \in L^{p^{\prime}}\left(\mathbb{R}^{N}\right)$ with $\operatorname{supp}(u) \subset B_{R}$ and $\operatorname{supp}(v) \subset \mathbb{R}^{N} \backslash B_{R+r}$,

$$
\left|\int_{\mathbb{R}^{N}} u \mathbf{R} v \mathrm{~d} x\right| \leq D r^{-\lambda_{p}}\|u\|_{p^{\prime}}\|v\|_{p^{\prime}}, \quad \text { where } \quad \lambda_{p}=\frac{N-1}{2}-\frac{N+1}{p} .
$$

The proof uses the decomposition $\Psi=\Psi_{1}+\Psi_{2}$ introduced in 2.18 for the fundamental solution of the Helmholtz equation. The dominant term in the estimate comes from the convolution with $\Psi_{1}$ and is obtained as follows. First remark that the Fourier transforms $\widehat{\Psi_{1}}$ and $\widehat{\Psi_{1}} \widehat{\varphi}$ coincide, for any $\varphi$ satisfying $\widehat{\varphi} \equiv 1$ on the set $\left\{|| \xi|-1| \leq \frac{1}{4}\right\}$. Choosing such a $\varphi$ for which in addition $\operatorname{supp}(\widehat{\varphi})$ is contained in $\left\{|| \xi|-1| \leq \frac{1}{2}\right\}$, one can replace $v$ with $\varphi * v$ and apply the result in [31, proposition 3.3] to the convolution $\left(1_{M_{r}} \Psi_{1}\right) *(\varphi * v)$ giving the asserted decay rate. The remaining convolution terms are estimated using Young's inequality and these only give lower order contributions. 
Based on this estimate, we prove a technical result which will be used in to deal with a remainder term in an estimate derived from the Hardy-Littlewood-Sobolev inequality.

Lemma 2.8. Let $\left(z_{n}\right)_{n} \subset L^{2^{+}}\left(\mathbb{R}^{N}\right)$ be a bounded sequence. Then, for every $\varepsilon>0$, there exists $\rho_{\varepsilon}>0$ such that

$$
\liminf _{n \rightarrow \infty}\left|\int_{\mathbb{R}^{N}} \mathbb{1}_{B_{\rho}} z_{n} \mathbf{R}\left(\mathbb{1}_{M_{\rho}} z_{n}\right) \mathrm{d} x\right|<\varepsilon, \quad \text { for all } \rho \geq \rho_{\varepsilon} .
$$

Here, $M_{\rho}:=\mathbb{R}^{N} \backslash B_{\rho}$.

Proof. Let $\zeta:=\sup \left\{\left\|z_{n}\right\|_{2^{+}}: n \in \mathbb{N}\right\}$. We first see that by lemma 2.7 there is a constant $D=D(N)>0$ such that

$$
\left|\int_{\mathbb{R}^{N}} \mathbb{1}_{B_{\rho}} z_{n} \mathbf{R}\left(\mathbb{1}_{M_{2 \rho}} z_{n}\right) \mathrm{d} x\right| \leq D \zeta^{2} \rho^{-\frac{1}{N}}, \quad \text { for all } n \in \mathbb{N} \text { and every } \rho \geq 1 .
$$

Hence, setting $\rho_{0}:=\max \left\{1,\left(\frac{2 D \zeta^{2}}{\varepsilon}\right)^{N}\right\}$ we find

$$
\left|\int_{\mathbb{R}^{N}} \mathbb{1}_{B_{\rho}} z_{n} \mathbf{R}\left(\mathbb{1}_{M_{2 \rho}} z_{n}\right) \mathrm{d} x\right| \leq \frac{\varepsilon}{2}, \quad \text { for all } n \in \mathbb{N} \text { and every } \rho \geq \rho_{0} .
$$

Next, we choose $\eta>0$ such that $\eta<\left(\frac{\varepsilon}{2 C_{0} \zeta}\right)^{2^{+}}$, where $C_{0}>0$ is such that 2.17) holds, and we claim that

$$
\exists \rho_{1}>0 \text { such that } \liminf _{n \rightarrow \infty} \int_{B_{2 \rho} \backslash B_{\rho}}\left|z_{n}\right|^{2^{+}} \mathrm{d} x<\eta, \quad \text { for all } \rho \geq \rho_{1} .
$$

Suppose this is not the case. Then, for every $k \in \mathbb{N}$ we can find a radius $\rho_{k} \geq k$ and an index $n_{0}(k) \in \mathbb{N}$ for which

$$
\int_{B_{2 \rho_{k} \backslash} \backslash B_{\rho_{k}}}\left|z_{n}\right|^{2^{+}} \mathrm{d} x \geq \eta, \quad \text { for all } n \geq n_{0}(k) .
$$

Moreover, we can assume without loss of generality that $n_{0}(k+1) \geq n_{0}(k)$ and $\rho_{k+1} \geq 2 \rho_{k}$. For each $\ell \in \mathbb{N}$, it follows that

$$
\zeta^{2^{+}} \geq \int_{\mathbb{R}^{N}}\left|z_{n}\right|^{2^{+}} \mathrm{d} x \geq \sum_{k=1}^{\ell} \int_{B_{2 \rho_{k}} \backslash B_{\rho_{k}}}\left|z_{n}\right|^{2^{+}} \mathrm{d} x \geq \ell \eta, \quad \text { for all } n \geq n_{0}(\ell) .
$$

For $\ell$ large enough we obtain a contradiction, and the claim is proven. 
As a consequence of the above results, we can write for $\rho \geq \rho_{\varepsilon}:=\max \left\{\rho_{0}, \rho_{1}\right\}$,

$$
\begin{aligned}
& \left|\int_{\mathbb{R}^{N}} \mathbb{1}_{B_{\rho}} z_{n} \mathbf{R}\left(\mathbb{1}_{M_{\rho}} z_{n}\right) \mathrm{d} x\right| \\
& \leq\left|\int_{\mathbb{R}^{N}} \mathbb{1}_{B_{\rho}} z_{n} \mathbf{R}\left(\mathbb{1}_{M_{2 \rho}} z_{n}\right) \mathrm{d} x\right|+\left|\int_{\mathbb{R}^{N}} \mathbb{1}_{B_{\rho}} z_{n} \mathbf{R}\left(\mathbb{1}_{B_{2 \rho} \backslash B_{\rho}} z_{n}\right) \mathrm{d} x\right| \\
& \leq \frac{\varepsilon}{2}+C_{0} \zeta\left\|\mathbb{1}_{B_{2 \rho} \backslash B_{\rho}} z_{n}\right\|_{2^{+}},
\end{aligned}
$$

using Hölder's inequality and the resolvent estimate (2.17). The conclusion then follows from the claim 2.29).

\section{Existence via the dual variational method}

We follow the path established in [31] and use a dual variational framework to find nontrivial solutions for the problem

$$
-\Delta u-u=Q(x)|u|^{2^{*}-2} u, \quad u \in W^{2,2^{*}}\left(\mathbb{R}^{N}\right),
$$

where $Q \in L^{\infty}\left(\mathbb{R}^{N}\right)$ is a nonnegative function which is not identically zero. Setting $v=$ $Q^{\frac{1}{2^{+}}}|u|^{2^{*}-2} u$, we shall study the fixed-point problem

$$
|v|^{2^{+}-2} v=Q^{\frac{1}{2^{*}}} \mathbf{R}\left(Q^{\frac{1}{2^{*}}} v\right), \quad v \in L^{2^{+}}\left(\mathbb{R}^{N}\right),
$$

where $\mathbf{R}$ denotes the resolvent Helmholtz operator defined in $\S 2.2$. For the Birman-Schwinger type operators associated to the Helmholtz and Laplace resolvents respectively, we introduce the notation

$$
\mathbf{A}_{Q} v:=Q^{\frac{1}{2^{*}}} \mathbf{R}\left(Q^{\frac{1}{2^{*}}} v\right) \quad \text { and } \quad \mathbf{G}_{Q} v:=Q^{\frac{1}{2^{*}}} \mathbf{R}_{0}\left(Q^{\frac{1}{2^{*}}} v\right), \quad v \in L^{2^{+}}\left(\mathbb{R}^{N}\right) .
$$

We consider the functional

$$
J_{Q}(v):=\frac{1}{2^{+}} \int_{\mathbb{R}^{N}}|v|^{2^{+}} \mathrm{d} x-\frac{1}{2} \int_{\mathbb{R}^{N}} v \mathbf{A}_{Q} v \mathrm{~d} x, \quad \text { for } v \in L^{2^{+}}\left(\mathbb{R}^{N}\right) .
$$

It is known that $J \in \mathcal{C}^{1}\left(L^{2^{+}}\left(\mathbb{R}^{N}\right), \mathbb{R}\right.$ ) and from the symmetry of $\mathbf{A}_{Q}$ (cf. [31, lemma 4.1]) we have

$$
J_{Q}^{\prime}(v) w=\int_{\mathbb{R}^{N}}\left(|v|^{2^{+}-2} v-\mathbf{A}_{Q} v\right) w \mathrm{~d} x, \quad \text { for all } v, w \in L^{2^{+}}\left(\mathbb{R}^{N}\right) .
$$

We detect solutions of 2.30 by finding critical points of the functional $J_{Q}$. Indeed, for $v \in$ $L^{2^{+}}\left(\mathbb{R}^{N}\right)$ we have $J_{Q}^{\prime}(v)=0$ if and only if $v$ satisfies 2.31). Setting $u=\mathbf{R}\left(Q^{\frac{1}{2^{*}}} v\right)$, we see that $u$ solves $u=\mathbf{R}\left(Q|u|^{2^{*}-2} u\right)$. Any solution of this integral equations has the following properties: 
Lemma 2.9 (Special case of [31, lemma 4.3]). Let $Q \in L^{\infty}\left(\mathbb{R}^{N}\right)$ and consider a solution $u \in L^{2^{*}}\left(\mathbb{R}^{N}\right)$ of $u=\mathbf{R}\left(Q|u|^{2^{*}-2} u\right)$. Then, $u \in W^{2, q}\left(\mathbb{R}^{N}\right)$ for all $2^{*} \leq q<\infty$ and $u$ is a strong solution of 2.30). Moreover, $u$ is the real part of a function $\widetilde{u}$ which satisfies Sommerfeld's outgoing radiation condition in the form

$$
\lim _{R \rightarrow \infty} \frac{1}{R} \int_{B_{R}}\left|\nabla \widetilde{u}(x)-\imath \widetilde{u}(x) \frac{x}{|x|}\right|^{2} \mathrm{~d} x=0 .
$$

In addition, $u$ satisfies the following asymptotic relation

$$
\lim _{R \rightarrow \infty} \frac{1}{R} \int_{B_{R}}\left|u(x)-\sqrt{\frac{\pi}{2}} \operatorname{Re}\left[\frac{e^{i|x|-\frac{i(N-3) \pi}{4}}}{|x|^{\frac{N-1}{2}}} \mathcal{F}\left(Q|u|^{2^{*}-2} u\right)\left(\frac{x}{|x|}\right)\right]\right|^{2} \mathrm{~d} x=0 .
$$

As shown in [31, lemma 4.2], the functional $J_{Q}$ has the mountain pass geometry, i.e.

(MP1) there exists $\delta>0$ and $\rho>0$ such that $J_{Q}(v) \geq \delta>0$,

for all $v \in L^{2^{+}}\left(\mathbb{R}^{N}\right)$ with $\|v\|_{2^{+}}=\rho$;

(MP2) there exists $v_{0} \in L^{2^{+}}\left(\mathbb{R}^{N}\right)$ such that $\left\|v_{0}\right\|_{2^{+}}>\rho$ and $J_{Q}\left(v_{0}\right)<0$.

The mountain pass level

$$
L_{Q}:=\inf _{P \in \mathcal{P}} \max _{t \in[0,1]} J_{Q}(P(t))
$$

where

$$
\mathcal{P}=\left\{P \in \mathcal{C}\left([0,1], L^{2^{+}}\left(\mathbb{R}^{N}\right)\right): P(0)=0 \text { and } J_{Q}(P(1))<0\right\},
$$

is therefore well defined, $0<L_{Q}<\infty$, and by the same arguments as in [29, Lemma 4.1], it can be characterized as the following infimum

$$
\begin{aligned}
L_{Q} & =\inf \left\{J_{Q}\left(t_{v} v\right): v \in L^{2^{+}}\left(\mathbb{R}^{N}\right) \text { with } \int_{\mathbb{R}^{N}} v \mathbf{A}_{Q} v \mathrm{~d} x>0\right\} \\
& =\inf \left\{\frac{1}{N}\left(\frac{\|v\|_{2^{+}}^{2}}{\int_{\mathbb{R}^{N}} v \mathbf{A}_{Q} v \mathrm{~d} x}\right)^{\frac{N}{2}}: v \in L^{2^{+}}\left(\mathbb{R}^{N}\right) \text { with } \int_{\mathbb{R}^{N}} v \mathbf{A}_{Q} v \mathrm{~d} x>0\right\} .
\end{aligned}
$$

Here, for $v \in L^{2^{+}}\left(\mathbb{R}^{N}\right)$ with $\int_{\mathbb{R}^{N}} v \mathbf{A}_{Q} v \mathrm{~d} x>0$,

$$
t_{v}=\left(\frac{\int_{\mathbb{R}^{N}}|v|^{2^{+}} \mathrm{d} x}{\int_{\mathbb{R}^{N}} v \mathbf{A}_{Q} v \mathrm{~d} x}\right)^{\frac{1}{2-2^{+}}}
$$

denotes the unique $t>0$ with the property $J_{Q}\left(t_{v} v\right)=\max _{t>0} J_{Q}(t v)$. Remarking that for every such $v, J_{Q}^{\prime}\left(t_{v} v\right) v=0$, we see that if $L_{Q}$ is achieved by some critical point of $J_{Q}$, then $L_{Q}$ 
coincides with the least-energy level, i.e.,

$$
L_{Q}=\inf \left\{J_{Q}(v): v \in L^{2^{+}}\left(\mathbb{R}^{N}\right) \backslash\{0\} \text { with } J_{Q}^{\prime}(v)=0\right\} .
$$

Following the terminology introduced in $[\mathbf{2 8}$, we will call a solution $u$ of the nonlinear Helmholtz equation 2.30) a dual ground state, if $u=\mathbf{R}\left(Q^{\frac{1}{2^{*}}} v\right)$ and $v \in L^{2^{+}}\left(\mathbb{R}^{N}\right)$ is a critical point of the functional $J_{Q}$ at the mountain pass level, i.e., $J_{Q}^{\prime}(v)=0$ and $J_{Q}(v)=L_{Q}$. As a consequence of the discussion at the beginning of the section, every dual ground state $u$ has the properties stated in Lemma 2.9.

3.1. Palais-Smale sequences. In this section, we investigate the properties of PalaisSmale sequences for the functional $J_{Q}$. Recall that a sequence $\left(v_{n}\right)_{n} \subset L^{2^{+}}\left(\mathbb{R}^{N}\right)$ is called a Palais-Smale sequence for $J_{Q}$ if $\left(J_{Q}\left(v_{n}\right)\right)_{n}$ is bounded and $\left\|J_{Q}^{\prime}\left(v_{n}\right)\right\|_{*} \rightarrow 0$ as $n \rightarrow \infty$. Here, $\|\cdot\|_{*}$ denotes the dual norm to $\|\cdot\|_{2^{+}}$. If, in addition, $J_{Q}\left(v_{n}\right) \rightarrow \beta$ as $n \rightarrow \infty$ for some $\beta \in \mathbb{R},\left(v_{n}\right)_{n}$ is called a $(P S)_{\beta}$-sequence for $J_{Q}$. We start by considering sequences which satisfy a localized version of the above property. For this purpose, we introduce the following piece of notation: If $v \in L^{2^{+}}\left(\mathbb{R}^{N}\right)$, we let $J_{Q}^{\prime}(v) \mathbb{1}_{B_{r}}$ denote the continuous linear form $w \mapsto J_{Q}^{\prime}(v)\left(\mathbb{1}_{B_{r}} w\right)$ on $L^{2^{+}}\left(\mathbb{R}^{N}\right)$.

Lemma 2.10. Let $\left(v_{n}\right)_{n} \subset L^{2^{+}}\left(\mathbb{R}^{N}\right)$ be a bounded sequence such that, for all $r>0,\left\|J_{Q}^{\prime}\left(v_{n}\right) \mathbb{1}_{B_{r}}\right\|_{*} \rightarrow$ 0 as $n \rightarrow \infty$. Then, up to a subsequence,

(i) $\left(v_{n}\right)_{n}$ has a weak limit $v \in L^{2^{+}}\left(\mathbb{R}^{N}\right)$.

(ii) For all $1 \leq q<2^{+}$and all $r>0$, we have $\mathbb{1}_{B_{r}} v_{n} \rightarrow \mathbb{1}_{B_{r}} v$, strongly in $L^{q}\left(\mathbb{R}^{N}\right)$ and $v_{n} \rightarrow v$ a.e. on $\mathbb{R}^{N}$, as $n \rightarrow \infty$.

(iii) $J_{Q}^{\prime}(v)=0$.

(iv) As $n \rightarrow \infty$, we have for all $r>0$,

$$
\left\|\mathbb{1}_{B_{r}}\left(v_{n}-v\right)\right\|_{2^{+}}^{2^{+}}=\int_{\mathbb{R}^{N}} \mathbb{1}_{B_{r}}\left(v_{n}-v\right) \mathbf{A}_{Q}\left(v_{n}-v\right) \mathrm{d} x+o(1) .
$$

Proof. Since $\left(v_{n}\right)_{n}$ is bounded in $L^{2^{+}}\left(\mathbb{R}^{N}\right)$, there exists $v \in L^{2^{+}}\left(\mathbb{R}^{N}\right)$ and a subsequence which we still denote by $\left(v_{n}\right)_{n}$ such that $v_{n} \rightarrow v$ weakly. This proves (i). From now on, we restrict to this particular subsequence. To prove (ii), let $r>0,1 \leq t<2^{*}$ and $\varphi \in \mathcal{C}_{c}^{\infty}\left(\mathbb{R}^{N}\right)$. 
For $n, m \in \mathbb{N}$ we have

$$
\begin{aligned}
& \left|\int_{\mathbb{R}^{N}}\left(\mathbb{1}_{B_{r}}\left|v_{n}\right|^{2^{+}-2} v_{n}-\mathbb{1}_{B_{r}}\left|v_{m}\right|^{2^{+}-2} v_{m}\right) \varphi \mathrm{d} x\right| \\
& =\left|\left[J_{Q}^{\prime}\left(v_{n}\right)-J_{Q}^{\prime}\left(v_{m}\right)\right]\left(\mathbb{1}_{B_{r}} \varphi\right)+\int_{\mathbb{R}^{N}} \mathbb{1}_{B_{r}} \varphi \mathbf{A}_{Q}\left(v_{n}-v_{m}\right) \mathrm{d} x\right| \\
& \leq\left\|J_{Q}^{\prime}\left(v_{n}\right) \mathbb{1}_{B_{r}}-J_{Q}^{\prime}\left(v_{m}\right) \mathbb{1}_{B_{r}}\right\|_{*}\left\|\mathbb{1}_{B_{r}} \varphi\right\|_{2^{+}}+\left\|\mathbb{1}_{B_{r}} \mathbf{A}_{Q}\left(v_{n}-v_{m}\right)\right\|_{t}\|\varphi\|_{t^{\prime}} \\
& \leq C\left[\left\|J_{Q}^{\prime}\left(v_{n}\right) \mathbb{1}_{B_{r}}\right\|_{*}+\left\|J_{Q}^{\prime}\left(v_{m}\right) \mathbb{1}_{B_{r}}\right\|_{*}\right]\|\varphi\|_{t^{\prime}} \\
& \quad+\|Q\|_{\infty}^{\frac{1}{2^{*}}}\left\|\mathbb{1}_{B_{r}} \mathbf{R}\left(Q^{\frac{1}{2^{*}}}\left(v_{n}-v_{m}\right)\right)\right\|_{t}\|\varphi\|_{t^{\prime}},
\end{aligned}
$$

where the constant $C>0$ depends on $N$ and $r$. The first expression in the last line vanishes as $n, m \rightarrow \infty$, by assumption, and the second one also vanishes due to lemma 2.4. Therefore, arguing by density, we find that $\left(\mathbb{1}_{B_{r}}\left|v_{n}\right|^{2^{+}-2} v_{n}\right)_{n}$ is a Cauchy sequence in $L^{t}\left(\mathbb{R}^{N}\right)$. Since the mapping $N: L^{t}\left(\mathbb{R}^{N}\right) \rightarrow L^{\frac{t}{2^{*}-1}}\left(\mathbb{R}^{N}\right)$ given by $N(u):=|u|^{2^{*}-2} u$ is well defined and Lipschitz continuous, it follows that $\left(\mathbb{1}_{B_{r}} v_{n}\right)_{n}=\left(N\left(\mathbb{1}_{B_{r}}\left|v_{n}\right|^{2^{+}-2} v_{n}\right)\right)_{n}$ is a Cauchy sequence in $L^{q}\left(\mathbb{R}^{N}\right)$ for all $1 \leq q<2^{+}$. Since these spaces are complete, and since $\mathbb{1}_{B_{r}} v_{n} \rightarrow \mathbb{1}_{B_{r}} v$ in each of these spaces, we obtain the desired strong convergence $\mathbb{1}_{B_{r}} v_{n} \rightarrow \mathbb{1}_{B_{r}} v$ in $L^{q}\left(\mathbb{R}^{N}\right)$ for all $1 \leq q<2^{+}$. Going to a subsequence, we also have the pointwise convergence $v_{n}(x) \rightarrow v(x)$ as $n \rightarrow \infty$, for almost every $x \in \mathbb{R}^{N}$. Assertion (iii) now follows from (ii), since for $\varphi \in \mathcal{C}_{c}^{\infty}\left(\mathbb{R}^{N}\right)$ and $r>0$ such that $\operatorname{supp}(\varphi) \subset B_{r}$, we have

$$
\begin{aligned}
J_{Q}^{\prime}(v) \varphi & =\int_{\mathbb{R}^{N}} \mathbb{1}_{B_{r}}|v|^{2^{+}-2} v \varphi \mathrm{d} x-\int_{\mathbb{R}^{N}} \mathbb{1}_{B_{r}} \varphi \mathbf{A}_{Q} v \mathrm{~d} x \\
& =\lim _{n \rightarrow \infty}\left[\int_{\mathbb{R}^{N}} \mathbb{1}_{B_{r}}\left|v_{n}\right|^{2^{+}-2} v_{n} \varphi \mathrm{d} x-\int_{\mathbb{R}^{N}} \mathbb{1}_{B_{r}} \varphi \mathbf{A}_{Q} v_{n} \mathrm{~d} x\right] \\
& =\lim _{n \rightarrow \infty} J_{Q}^{\prime}\left(v_{n}\right) \mathbb{1}_{B_{r}} \varphi=0 .
\end{aligned}
$$

To prove assertion (iv) we use the assumption $\left\|J_{Q}^{\prime}\left(v_{n}\right) \mathbb{1}_{B_{r}}\right\|_{*} \rightarrow 0$ as $n \rightarrow \infty$ and write

$$
\begin{array}{r}
o(1)=J_{Q}^{\prime}\left(v_{n}\right) \mathbb{1}_{B_{r}} v_{n}=\left\|\mathbb{1}_{B_{r}} v_{n}\right\|_{2^{+}}^{2^{+}}-\int_{\mathbb{R}^{N}} \mathbb{1}_{B_{r}} v_{n} \mathbf{A}_{Q} v_{n} \mathrm{~d} x \\
=\left\|\mathbb{1}_{B_{r}} v_{n}\right\|_{2^{+}}^{2^{+}}-\int_{\mathbb{R}^{N}} \mathbb{1}_{B_{r}}\left(v_{n}-v\right) \mathbf{A}_{Q}\left(v_{n}-v\right) \mathrm{d} x \\
-\int_{\mathbb{R}^{N}} \mathbb{1}_{B_{r}} v_{n} \mathbf{A}_{Q} v \mathrm{~d} x-\int_{\mathbb{R}^{N}} \mathbb{1}_{B_{r}} v \mathbf{A}_{Q}\left(v_{n}-v\right) \mathrm{d} x .
\end{array}
$$


The last expression vanishes as $n \rightarrow \infty$, since $v_{n} \rightarrow v$ in $L^{2^{+}}\left(\mathbb{R}^{N}\right)$ and since $\mathbf{A}_{Q}: L^{2^{+}}\left(\mathbb{R}^{N}\right)$ $\rightarrow L^{2^{*}}\left(\mathbb{R}^{N}\right)$ is continuous. Furthermore, using (iii), the weak convergence $v_{n} \rightarrow v$ and the Brézis-Lieb lemma [70, lemma 1.32], which applies due to (ii), we obtain

$$
\begin{aligned}
& \left\|\mathbb{1}_{B_{r}} v_{n}\right\|_{2^{+}}^{2^{+}}-\int_{\mathbb{R}^{N}} \mathbb{1}_{B_{r}} v_{n} \mathbf{A}_{Q} v \mathrm{~d} x \\
& =\left\|\mathbb{1}_{B_{r}} v_{n}\right\|_{2^{+}}^{2^{+}}-\left(\left\|\mathbb{1}_{B_{r}} v\right\|_{2^{+}}^{2^{+}}-\int_{\mathbb{R}^{N}} \mathbb{1}_{B_{r}} v \mathbf{A}_{Q} v \mathrm{~d} x\right)-\int_{\mathbb{R}^{N}} \mathbb{1}_{B_{r}} v_{n} \mathbf{A}_{Q} v \mathrm{~d} x \\
& =\left\|\mathbb{1}_{B_{r}}\left(v_{n}-v\right)\right\|_{2^{+}}^{2^{+}}+o(1), \quad \text { as } n \rightarrow \infty .
\end{aligned}
$$

Substituting in (2.37), the desired conclusion follows.

In the above proof, the fact that the operator $\mathbb{1}_{B_{r}} \mathbf{R}: L^{2^{+}}\left(\mathbb{R}^{N}\right) \rightarrow L^{t}\left(\mathbb{R}^{N}\right)$ is compact for $1 \leq t<2^{*}$ was essential. For $t=2^{*}$, the compactness does not hold anymore and therefore the assertion (ii) is false in this case. However, in view of proposition 2.5, this is only caused by the noncompactness of the operator $\mathbb{1}_{B_{r}} \mathbf{R}_{0}: L^{2^{+}}\left(\mathbb{R}^{N}\right) \rightarrow L^{2^{*}}\left(\mathbb{R}^{N}\right)$ associated to the fundamental solution of Laplace's equation. In the next result, we prove that local strong convergence can be restored, provided the mountain pass level $L_{Q}$ lies below the threshold value given by the least-energy level $L_{Q}^{*}$ of the functional

$$
J_{Q}^{*}(v):=\frac{1}{2^{+}} \int_{\mathbb{R}^{N}}|v|^{2^{+}} \mathrm{d} x-\frac{1}{2} \int_{\mathbb{R}^{N}} v \mathbf{G}_{q} v \mathrm{~d} x,
$$

where $\mathbf{G}_{q} v:=q^{\frac{1}{2^{*}}} \mathbf{R}_{0}\left(q^{\frac{1}{2^{*}}} v\right)$ for $q=\|Q\|_{\infty}$. As mentioned in the introduction, this functional arises after passing to the limit of a suitable rescaling of $J_{Q}$. The least-energy level $L_{Q}^{*}$ can be characterized by a formula similar to (2.34), namely

$$
L_{Q}^{*}=\inf \left\{\frac{1}{N}\left(\frac{\|v\|_{2^{+}}^{2}}{\|Q\|_{\infty}^{\frac{2}{2^{*}}} \int_{\mathbb{R}^{N}} v \mathbf{R}_{0} v \mathrm{~d} x}\right)^{\frac{N}{2}}: v \in L^{2^{+}}\left(\mathbb{R}^{N}\right) \backslash\{0\}\right\} .
$$

We note incidentally that it can be expressed in terms of the optimal constant $S$ for the Sobolev inequality in $\mathbb{R}^{N}$,

$$
\|\nabla u\|_{2}^{2} \geq S\|u\|_{2^{*}}^{2}, \quad \text { for all } u \in L^{2^{*}}\left(\mathbb{R}^{N}\right) \text { with } \nabla u \in L^{2}\left(\mathbb{R}^{N}\right) .
$$

Indeed, it is known (see [46] and also [23) that the Sobolev inequality is dual to the HardyLittlewood-Sobolev inequality

$$
\int_{\mathbb{R}^{N}} v \mathbf{R}_{0} v \mathrm{~d} x \leq S^{-1}\|v\|_{2^{+}}^{2}
$$


and that the optimal constants are inverse to each other. Hence, we obtain

$$
L_{Q}^{*}=\frac{S^{\frac{N}{2}}}{N\|Q\|_{\infty}^{\frac{N-2}{2}}} .
$$

Proposition 2.11. Let $Q \in L^{\infty}\left(\mathbb{R}^{N}\right) \backslash\{0\}$ have the form $Q=Q_{\text {per }}+Q_{0}$, for some $Q_{\text {per }}, Q_{0} \geq 0$ such that $Q_{\text {per }}$ is $\mathbb{Z}^{N}$-periodic and $Q_{0}(x) \rightarrow 0$ as $|x| \rightarrow \infty$.

If $\left(v_{n}\right)_{n} \subset L^{2^{+}}\left(\mathbb{R}^{N}\right)$ is a $(P S)_{\beta}$-sequence for $J_{Q}$ such that $\beta=L_{Q}<L_{Q}^{*}$, then there exists $w \in L^{2^{+}}\left(\mathbb{R}^{N}\right), w \neq 0$, such that $J_{Q}^{\prime}(w)=0$ and $J_{Q}(w)=L_{Q}$.

Proof. Since $\left(v_{n}\right)_{n}$ is a $(\mathrm{PS})_{\beta}$-sequence for $J_{Q}$ with $\beta>0$, it is bounded (see [31, lemma $4.2])$. Using lemma 2.10, we find

$$
\begin{aligned}
\lim _{n \rightarrow \infty} \int_{\mathbb{R}^{N}} Q^{\frac{1}{2^{*}}} v_{n} \mathbf{R}\left(Q^{\frac{1}{2^{*}}} v_{n}\right) \mathrm{d} x & =\left(\frac{1}{2^{+}}-\frac{1}{2}\right)^{-1} \lim _{n \rightarrow \infty}\left[J_{Q}\left(v_{n}\right)-\frac{1}{2^{+}} J_{Q}^{\prime}\left(v_{n}\right) v_{n}\right] \\
& =N \beta>0 .
\end{aligned}
$$

Hence, the nonvanishing theorem 2.6 gives the existence of a sequence $\left(x_{n}\right)_{n} \subset \mathbb{R}^{N}$ and of constants $R, \zeta>0$ such that, up to a subsequence,

$$
\int_{B_{R}\left(x_{n}\right)}\left|v_{n}\right|^{2^{+}} \mathrm{d} x \geq\|Q\|_{\infty^{-\frac{2^{*}}{2^{*}}}} \int_{B_{R}\left(x_{n}\right)}\left|Q^{\frac{1}{2^{*}}} v_{n}\right|^{2^{+}} \mathrm{d} x \geq\|Q\|_{\infty}^{-\frac{2^{+}}{2^{*}}} \zeta>0, \quad \text { for all } n .
$$

Moreover, we may assume that $\left(x_{n}\right)_{n} \subset \mathbb{Z}^{N}$ by making $R$ larger if necessary. We now distinguish two cases.

Case 1: $\left|x_{n}\right| \rightarrow \infty$, for a subsequence.

Let us restrict to this subsequence, setting $w_{n}:=v_{n}\left(\cdot+x_{n}\right)$ for all $n$. We shall use lemma 2.10 for the sequence $\left(w_{n}\right)_{n}$ and with $Q$ replaced with $Q_{\text {per }}$. We therefore need to check that $\left\|J_{Q_{\text {per }}}^{\prime}\left(w_{n}\right) \mathbb{1}_{B_{r}}\right\|_{*} \rightarrow 0$ as $n \rightarrow \infty$, for all $r>0$. For this, observe that for $r>0$ and $\varphi \in \mathcal{C}_{c}^{\infty}\left(\mathbb{R}^{N}\right)$ we have

$$
\begin{aligned}
J_{Q_{\mathrm{per}}}^{\prime}\left(w_{n}\right) \mathbb{1}_{B_{r}} \varphi= & J_{Q}^{\prime}\left(v_{n}\right) \mathbb{1}_{B_{r}\left(x_{n}\right)} \varphi\left(\cdot-x_{n}\right) \\
& +\int_{\mathbb{R}^{N}} \mathbb{1}_{B_{r}} \varphi\left(\mathbf{A}_{Q\left(\cdot+x_{n}\right)}-\mathbf{A}_{Q_{\mathrm{per}}\left(\cdot+x_{n}\right)}\right) w_{n} \mathrm{~d} x,
\end{aligned}
$$

using the fact that $Q_{\text {per }}$ is invariant under $\mathbb{Z}^{N}$-translations. Since $\left(v_{n}\right)_{n}$ is a Palais-Smale sequence, the first term in the right-hand side goes to zero uniformly for $\|\varphi\|_{2^{+}} \leq 1$. The 
second term can be estimated as follows.

$$
\begin{aligned}
& \left|\int_{\mathbb{R}^{N}} \mathbb{1}_{B_{r}} \varphi\left(\mathbf{A}_{Q\left(\cdot+x_{n}\right)}-\mathbf{A}_{Q_{\mathrm{per}}\left(\cdot+x_{n}\right)}\right) w_{n} \mathrm{~d} x\right| \\
& =\left|\int_{\mathbb{R}^{N}} \mathbb{1}_{B_{r}} \varphi\left(Q^{\frac{1}{2^{*}}}\left(\cdot+x_{n}\right)-Q_{\mathrm{per}}^{\frac{1}{2^{*}}}\left(\cdot+x_{n}\right)\right) \mathbf{R}\left[\left(Q^{\frac{1}{2^{*}}}\left(\cdot+x_{n}\right)+Q_{\mathrm{per}}^{\frac{1}{2^{*}}}\left(\cdot+x_{n}\right)\right) w_{n}\right] \mathrm{d} x\right| \\
& \leq 2 C_{0}\|Q\|_{\infty}^{\frac{1}{2^{*}}}\|\varphi\|_{2^{+}}\left\|w_{n}\right\|_{2^{+}}\left\|\mathbb{1}_{B_{r}}\left(Q^{\frac{1}{2^{*}}}\left(\cdot+x_{n}\right)-Q_{\operatorname{per}}^{\frac{1}{2^{*}}}\left(\cdot+x_{n}\right)\right)\right\|_{\infty},
\end{aligned}
$$

where $C_{0}>0$ is given by (2.17). Moreover, by assumption, $Q(x)-Q_{\text {per }}(x)=Q_{0}(x) \rightarrow 0$ as $|x| \rightarrow \infty$. Thus, since $\left|x_{n}\right| \rightarrow \infty$ as $n \rightarrow \infty$ and $Q, Q_{\text {per }} \geq 0$ are bounded functions, it follows that

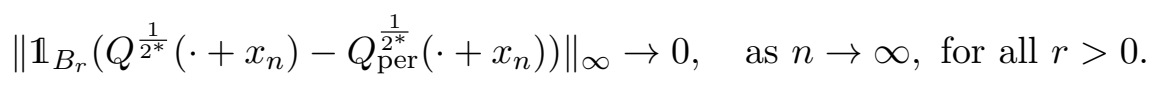

Combining (2.43), 2.44) and 2.45, we find that $\left\|J_{Q_{\mathrm{per}}}^{\prime}\left(w_{n}\right) \mathbb{1}_{B_{r}}\right\|_{*} \rightarrow 0$, as $n \rightarrow \infty$. Therefore the conditions of lemma 2.10 are fulfilled and, going to a subsequence, we obtain $w_{n} \rightarrow w$ in $L^{2^{+}}\left(\mathbb{R}^{N}\right)$ and $w_{n} \rightarrow w$ a.e. on $\mathbb{R}^{N}$, for some $w \in L^{2^{+}}\left(\mathbb{R}^{N}\right)$ which satisfies $J_{Q_{\text {per }}}^{\prime}(w)=0$.

Furthermore, from 2.36 we infer that, as $n \rightarrow \infty$,

$$
\begin{aligned}
\left\|\mathbb{1}_{B_{r}}\left(w_{n}-w\right)\right\|_{2^{+}}^{2^{+}} & =\int_{\mathbb{R}^{N}} \mathbb{1}_{B_{r}}\left(w_{n}-w\right) \mathbf{A}_{Q_{\mathrm{per}}}\left(w_{n}-w\right) \mathrm{d} x+o(1) \\
= & \int_{\mathbb{R}^{N}} \mathbb{1}_{B_{r}}\left(w_{n}-w\right) \mathbf{A}_{Q_{\mathrm{per}}}\left[\mathbb{1}_{B_{r}}\left(w_{n}-w\right)\right] \mathrm{d} x \\
& +\int_{\mathbb{R}^{N}} \mathbb{1}_{B_{r}}\left(w_{n}-w\right) \mathbf{A}_{Q_{\mathrm{per}}}\left[\mathbb{1}_{M_{r}}\left(w_{n}-w\right)\right] \mathrm{d} x+o(1),
\end{aligned}
$$

for all $r>0$, where $M_{r}:=\mathbb{R}^{N} \backslash B_{r}$.

For the first integral we obtain with proposition 2.5 and the characterization 2.38 of $L_{Q}^{*}$,

$$
\begin{aligned}
& \int_{\mathbb{R}^{N}} \mathbb{1}_{B_{r}}\left(w_{n}-w\right) \mathbf{A}_{Q_{\mathrm{per}}} {\left[\mathbb{1}_{B_{r}}\left(w_{n}-w\right)\right] \mathrm{d} x } \\
&= \int_{\mathbb{R}^{N}} \mathbb{1}_{B_{r}}\left(w_{n}-w\right) \mathbf{G}_{Q_{\mathrm{per}}}\left[\mathbb{1}_{B_{r}}\left(w_{n}-w\right)\right] \mathrm{d} x \\
& \quad+\int_{\mathbb{R}^{N}} \mathbb{1}_{B_{r}}\left(w_{n}-w\right)\left(\mathbf{A}_{Q_{\mathrm{per}}}-\mathbf{G}_{Q_{\mathrm{per}}}\right)\left[\mathbb{1}_{B_{r}}\left(w_{n}-w\right)\right] \mathrm{d} x \\
& \leq\|Q\|_{\infty}^{\frac{2}{2^{*}}} \int_{\mathbb{R}^{N}} \mathbb{1}_{B_{r}}\left|w_{n}-w\right| \mathbf{R}_{0}\left[\mathbb{1}_{B_{r}}\left|w_{n}-w\right|\right] \mathrm{d} x+o(1) \\
& \leq\left(N L_{Q}^{*}\right)^{-\frac{2}{N}}\left\|\mathbb{1}_{B_{r}}\left(w_{n}-w\right)\right\|_{2^{+}}^{2}+o(1), \quad \text { as } n \rightarrow \infty,
\end{aligned}
$$


where $\mathbf{G}_{Q_{\text {per }}}$ is given by 2.32 with $Q_{\text {per }}$ in place of $Q$. In addition, the Brézis-Lieb lemma implies

$$
\begin{aligned}
\left\|\mathbb{1}_{B_{r}}\left(w_{n}-w\right)\right\|_{2^{+}}^{2^{+}} & \leq\left\|w_{n}-w\right\|_{2^{+}}^{2^{+}}=\left\|w_{n}\right\|_{2^{+}}^{2^{+}}-\|w\|_{2^{+}}^{2^{+}}+o(1) \\
& \leq\left\|v_{n}\right\|_{2^{+}}^{2^{+}}+o(1)=\left(\frac{1}{2^{+}}-\frac{1}{2}\right)^{-1}\left(J_{Q}\left(v_{n}\right)-\frac{1}{2} J_{Q}^{\prime}\left(v_{n}\right) v_{n}\right)+o(1) \\
& =N \beta+o(1), \quad \text { as } n \rightarrow \infty
\end{aligned}
$$

since $\left(v_{n}\right)_{n}$ is a $(P S)_{\beta}$-sequence by assumption. Combining these two estimates, we obtain

$$
\begin{aligned}
& {\left[1-\left(\frac{\beta}{L_{Q}^{*}}\right)^{\frac{2}{N}}\right]\left\|\mathbb{1}_{B_{r}}\left(w_{n}-w\right)\right\|_{2^{+}}^{2^{+}}} \\
& \leq\left\|\mathbb{1}_{B_{r}}\left(w_{n}-w\right)\right\|_{2^{+}}^{2^{+}}-\left(N L_{Q}^{*}\right)^{-\frac{2}{N}}\left\|\mathbb{1}_{B_{r}}\left(w_{n}-w\right)\right\|_{2^{+}}^{2}+o(1) \\
& \leq\left\|\mathbb{1}_{B_{r}}\left(w_{n}-w\right)\right\|_{2^{+}}^{2^{+}}-\int_{\mathbb{R}^{N}} \mathbb{1}_{B_{r}}\left(w_{n}-w\right) \mathbf{A}_{Q_{\text {per }}}\left[\mathbb{1}_{B_{r}}\left(w_{n}-w\right)\right] \mathrm{d} x+o(1)
\end{aligned}
$$

as $n \rightarrow \infty$, and (2.46) gives for all $r>0$,

$$
\left[1-\left(\frac{\beta}{L_{Q}^{*}}\right)^{\frac{2}{N}}\right]\left\|\mathbb{1}_{B_{r}}\left(w_{n}-w\right)\right\|_{2^{+}}^{2^{+}} \leq \int_{\mathbb{R}^{N}} \mathbb{1}_{B_{r}}\left(w_{n}-w\right) \mathbf{A}_{Q_{\mathrm{per}}}\left[\mathbb{1}_{M_{r}}\left(w_{n}-w\right)\right] \mathrm{d} x+o(1),
$$

as $n \rightarrow \infty$, where the first factor on the left-hand side is strictly positive, since we are assuming $\beta<L_{Q}^{*}$.

Let us now suppose by contradiction that $\left(\mathbb{1}_{B_{r}} w_{n}\right)_{n}$ does not converge strongly to $\mathbb{1}_{B_{r}} w$ in $L^{2^{+}}\left(\mathbb{R}^{N}\right)$, for some fixed $r>0$. Then, passing to a subsequence, there exists $\varepsilon>0$ such that

$$
\left[1-\left(\frac{\beta}{L_{Q}^{*}}\right)^{\frac{2}{N}}\right]\left\|\mathbb{1}_{B_{r}}\left(w_{n}-w\right)\right\|_{2^{+}}^{2^{+}}>\varepsilon \quad \text { for all } n .
$$




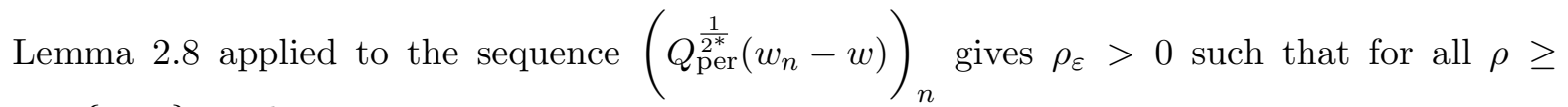
$\max \left\{\rho_{\varepsilon}, r\right\}$, we have

$$
\begin{aligned}
& \varepsilon>\liminf _{n \rightarrow \infty}\left|\int_{\mathbb{R}^{N}} \mathbb{1}_{B_{\rho}}\left(w_{n}-w\right) \mathbf{A}_{Q_{\mathrm{per}}}\left[\mathbb{1}_{M_{\rho}}\left(w_{n}-w\right)\right] \mathrm{d} x\right| \\
& \geq \liminf _{n \rightarrow \infty}\left[1-\left(\frac{\beta}{L_{Q}^{*}}\right)^{\frac{2}{N}}\right]\left\|\mathbb{1}_{B_{\rho}}\left(w_{n}-w\right)\right\|_{2^{+}}^{2^{+}}, \quad \text { using }(2.47) \\
& \geq \liminf _{n \rightarrow \infty}\left[1-\left(\frac{\beta}{L_{Q}^{*}}\right)^{\frac{2}{N}}\right]\left\|\mathbb{1}_{B_{r}}\left(w_{n}-w\right)\right\|_{2^{+}}^{2^{+}} \\
& \geq \varepsilon .
\end{aligned}
$$

This contradiction proves the strong convergence $\mathbb{1}_{B_{r}} w_{n} \rightarrow \mathbb{1}_{B_{r}} w$ in $L^{2^{+}}\left(\mathbb{R}^{N}\right)$ as $n \rightarrow \infty$, for all $r>0$. Using 2.42 we immediately deduce that $w \neq 0$. Moreover,

$$
\begin{aligned}
J_{Q_{\mathrm{per}}}(w) & =J_{Q_{\mathrm{per}}}(w)-\frac{1}{2} J_{Q_{\mathrm{per}}}^{\prime}(w) w=\frac{1}{N}\|w\|_{2^{+}}^{2^{+}} \\
& \leq \liminf _{n \rightarrow \infty} \frac{1}{N}\left\|w_{n}\right\|_{2^{+}}^{2^{+}}=\liminf _{n \rightarrow \infty} \frac{1}{N}\left\|v_{n}\right\|_{2^{+}}^{2^{+}} \\
& =\liminf _{n \rightarrow \infty}\left[J_{Q}\left(v_{n}\right)-\frac{1}{2} J_{Q}^{\prime}\left(v_{n}\right) v_{n}\right]=\beta=L_{Q} .
\end{aligned}
$$

Now, consider the function

$$
\widetilde{w}:=\left(\frac{Q_{\mathrm{per}}}{Q}\right)^{\frac{1}{2^{*}}} w .
$$

Since $Q=Q_{\text {per }}+Q_{0}$ with $Q_{0} \geq 0$, we find that $|\widetilde{w}| \leq|w|$. In particular, we have $\widetilde{w} \in L^{2^{+}}\left(\mathbb{R}^{N}\right)$ and by definition,

$$
\int_{\mathbb{R}^{N}} \widetilde{w} \mathbf{A}_{Q} \widetilde{w} \mathrm{~d} x=\int_{\mathbb{R}^{N}} w \mathbf{A}_{Q_{\mathrm{per}}} w \mathrm{~d} x=\|w\|_{2^{+}}^{2^{+}}>0,
$$

since $w$ is a nontrivial critical point of $J_{Q_{\mathrm{per}}}$. Hence, $\widetilde{w} \neq 0$ and, setting

$$
\tau:=\left(\frac{\int_{\mathbb{R}^{N}}|\widetilde{w}|^{2^{+}} \mathrm{d} x}{\int_{\mathbb{R}^{N}} \widetilde{w} \mathbf{A}_{Q} \widetilde{w} \mathrm{~d} x}\right)^{\frac{1}{2-2^{+}}}
$$

we find that $0<\tau \leq 1$ and $J_{Q}^{\prime}(\tau \widetilde{w}) \widetilde{w}=0$. In addition, since $|\widetilde{w}| \leq|w|$ we have

$$
J_{Q}(\tau \widetilde{w})=\frac{1}{N}\left(\frac{\|\widetilde{w}\|_{2^{+}}^{2}}{\int \widetilde{w} \mathbf{A}_{Q} \widetilde{w} \mathrm{~d} x}\right)^{\frac{N}{2}} \leq \frac{1}{N}\left(\frac{\|w\|_{2^{+}}^{2}}{\int_{\mathbb{R}^{N}} w \mathbf{A}_{Q_{\mathrm{per}}} w \mathrm{~d} x}\right)^{\frac{N}{2}}=J_{Q_{\mathrm{per}}}(w) .
$$


Therefore, 2.34 and 2.48 yield $J_{Q}(\tau \widetilde{w})=L_{Q}=J_{Q_{\mathrm{per}}}(w)$ and we deduce that $\tau=1$. We now claim that $\tau \widetilde{w}=\widetilde{w}$ is a critical point for $J_{Q}$. To prove this, let $\varphi \in L^{2^{+}}\left(\mathbb{R}^{N}\right)$ be arbitrarily given and choose $\delta>0$ such that

$$
\int_{\mathbb{R}^{N}}(\widetilde{w}+s \varphi) \mathbf{A}_{Q}(\widetilde{w}+s \varphi) \mathrm{d} x>0, \quad \text { for all } s \in[-\delta, \delta],
$$

and, for $s \in[-\delta, \delta]$, set

$$
t_{s}:=\left(\frac{\int_{\mathbb{R}^{N}}|\widetilde{w}+s \varphi|^{2^{+}} \mathrm{d} x}{\int_{\mathbb{R}^{N}}(\widetilde{w}+s \varphi) \mathbf{A}_{Q}(\widetilde{w}+s \varphi) \mathrm{d} x}\right)^{\frac{1}{2-2^{+}}} .
$$

Then we can write, using 2.34 , the property $J_{Q}(\widetilde{w})=J_{Q}(\tau \widetilde{w})=\max _{t>0} J_{Q}(t \widetilde{w})$ and the meanvalue theorem,

$$
\begin{aligned}
0 \leq J_{Q}\left(t_{s}(\widetilde{w}+s \varphi)\right)-J_{Q}(\widetilde{w}) & \leq J_{Q}\left(t_{s}(\widetilde{w}+s \varphi)\right)-J_{Q}\left(t_{s} \widetilde{w}\right) \\
& =J_{Q}^{\prime}\left(t_{s}(\widetilde{w}+s \sigma \varphi)\right) t_{s} s \varphi
\end{aligned}
$$

for some $\sigma \in[-1,1]$. Dividing by $s \neq 0$ and letting $s \rightarrow 0^{ \pm}$, we obtain $J_{Q}^{\prime}(\widetilde{w}) \varphi=0$, since $t_{s} \rightarrow 1$, as $s \rightarrow 0$. The proposition is proven in this case.

Case 2: $\left(x_{n}\right)_{n}$ is bounded. In this case, making $R$ again larger if necessary, we can assume that 2.42 holds with $x_{n}=0$ for all $n$.

Since $\left(v_{n}\right)_{n}$ is a $(\mathrm{PS})_{\beta}$-sequence, the assumptions of lemma 2.10 are satisfied. Thus, going to a subsequence, we obtain $v_{n} \rightarrow v$ in $L^{2^{+}}\left(\mathbb{R}^{N}\right)$ and $v_{n} \rightarrow v$ a.e. on $\mathbb{R}^{N}$, for some $v \in L^{2^{+}}\left(\mathbb{R}^{N}\right)$ which satisfies $J_{Q}^{\prime}(v)=0$. Replacing in 2.46) and the subsequent computations $Q_{\text {per }}$ with $Q$, $w_{n}$ with $v_{n}$ and $w$ with $v$, we obtain for all $r>0$,

$$
\left[1-\left(\frac{\beta}{L_{Q}^{*}}\right)^{\frac{2}{N}}\right]\left\|\mathbb{1}_{B_{r}}\left(v_{n}-v\right)\right\|_{2^{+}}^{2^{+}} \leq \int_{\mathbb{R}^{N}} \mathbb{1}_{B_{r}}\left(v_{n}-v\right) \mathbf{A}_{Q}\left[\mathbb{1}_{M_{r}}\left(v_{n}-v\right)\right] \mathrm{d} x+o(1),
$$

as $n \rightarrow \infty$, and the same contradiction argument as in Case 1 yields the strong convergence $\mathbb{1}_{B_{r}} v_{n} \rightarrow \mathbb{1}_{B_{r}} v$ as $n \rightarrow \infty$ in $L^{2^{+}}\left(\mathbb{R}^{N}\right)$, for all $r>0$. In particular, $v$ is a nontrivial critical point of $J_{Q}$ and the characterization 2.34 of the mountain-pass level $L_{Q}$ yields

$$
\begin{aligned}
L_{Q} \leq J_{Q}(v) & =J_{Q}(v)-\frac{1}{2} J_{Q}^{\prime}(v) v=\frac{1}{N}\|v\|_{2^{+}}^{2^{+}} \\
& \leq \liminf _{n \rightarrow \infty} \frac{1}{N}\left\|v_{n}\right\|_{2^{+}}^{2^{+}}=\liminf _{n \rightarrow \infty}\left[J_{Q}\left(v_{n}\right)-\frac{1}{2} J_{Q}^{\prime}\left(v_{n}\right) v_{n}\right]=\beta=L_{Q} .
\end{aligned}
$$

Hence, $J_{Q}(v)=L_{Q}$ and this concludes the proof.

REMARK 2.12. (i) When $Q \equiv Q_{0}$, Case 1 does not occur in the proof above. Moreover, the operator $\mathbf{A}_{Q}-\mathbf{G}_{Q}$ is itself compact, so that all arguments in the proof hold globally on $\mathbb{R}^{N}$. As a consequence, $J_{Q}$ satisfies the Palais-Smale condition at every level $0<\beta<L_{Q}^{*}$. 
(ii) In the case where $Q \equiv Q_{\text {per }}$, we have $\widetilde{w}=w$ in the above proof and the proposition is valid for any $0<\beta<L_{Q}^{*}$, except for the last assertion which should be replaced with $L_{Q} \leq J_{Q}(w) \leq \beta$.

3.2. Estimating the dual mountain-pass level. Our next result shows that in dimension $N \geq 4$, the mountain-pass level $L_{Q}$ lies below the critical threshold $L_{Q}^{*}$ if the coefficient $Q$ satisfies some flatness condition (see condition (Q) below). This additional condition seems to go back to the works of Escobar [25] and Egnell [24] (see also [14, Remark 1.2]).

To estimate $L_{Q}$, we shall use the functions

$$
v_{\varepsilon}(x):=(N(N-2) \varepsilon)^{\frac{N+2}{4}}\left(\frac{1}{\varepsilon+|x|^{2}}\right)^{\frac{N+2}{2}}, \quad \varepsilon>0 .
$$

It was shown by Lieb [45] (see also [46, theorem 4.3]) that, up to translation and multiplication by a constant, $v_{\varepsilon}, \varepsilon>0$ are the only optimizers of the Hardy-Littlewood-Sobolev inequality (2.40, i.e.,

$$
\int_{\mathbb{R}^{N}} v_{\varepsilon} \mathbf{R}_{0} v_{\varepsilon} \mathrm{d} x=S^{-1}\left\|v_{\varepsilon}\right\|_{2^{+}}^{2} .
$$

In addition, we have $v_{\varepsilon}=u_{\varepsilon}^{2^{*}-1}$, where

$$
u_{\varepsilon}(x):=(N(N-2) \varepsilon)^{\frac{N-2}{4}}\left(\frac{1}{\varepsilon+|x|^{2}}\right)^{\frac{N-2}{2}}, \quad \varepsilon>0,
$$

are the Aubin-Talenti instantons (see, e.g., [10]) that optimize the Sobolev inequality (2.39) and for which the following holds:

$$
\left\|\nabla u_{\varepsilon}\right\|_{L^{2}\left(\mathbb{R}^{N}\right)}^{2}=\left\|u_{\varepsilon}\right\|_{2^{*}}^{2^{*}}=S^{\frac{N}{2}}, \quad \text { for all } \varepsilon>0 .
$$

In particular, we deduce that

$$
\left\|v_{\varepsilon}\right\|_{2^{+}}=\left\|u_{\varepsilon}\right\|_{2^{*}}^{2^{*}-1}=S^{\frac{N+2}{4}}, \quad \text { for all } \varepsilon>0 .
$$

Proposition 2.13. Let $N \geq 4$ and consider $Q \in L^{\infty}\left(\mathbb{R}^{N}\right) \backslash\{0\}$ nonnegative. Assume further, that there exists $x_{0} \in \mathbb{R}^{N}$ with $Q\left(x_{0}\right)=\|Q\|_{\infty}$ and that

$$
Q\left(x_{0}\right)-Q(x)=o\left(\left|x-x_{0}\right|^{2}\right), \quad \text { as }\left|x-x_{0}\right| \rightarrow 0 .
$$

Then we have

$$
L_{Q}<L_{Q}^{*}
$$

Proof. Let us assume - without loss of generality - that $x_{0}=0$ and set $q:=\|Q\|_{\infty}$. We consider for $\varepsilon>0$ the dual instanton $v_{\varepsilon}$ given by (2.49) and put $v:=v_{1}$. Fix a cut-off function $\varphi \in \mathcal{C}_{c}^{\infty}\left(\mathbb{R}^{N}\right)$ with $0 \leq \varphi \leq 1$ on $\mathbb{R}^{N}, \varphi \equiv 1$ on $B_{1}(0)$ and $\varphi \equiv 0$ outside of $B_{2}(0)$. Setting for $\varepsilon>0, \alpha>0$,

$$
v_{\varepsilon, \alpha}:=\varphi_{\alpha} v_{\varepsilon}, \quad \text { where } \varphi_{\alpha}(x):=\varphi\left(\frac{x}{\alpha}\right),
$$


we shall estimate the ratio

$$
\frac{\left\|v_{\varepsilon, \alpha}\right\|_{2^{+}}^{2}}{\int_{\mathbb{R}^{N}} v_{\varepsilon, \alpha} \mathbf{A}_{Q} v_{\varepsilon, \alpha} \mathrm{d} x}
$$

and we first look at the quadratic form $\int_{\mathbb{R}^{N}} v_{\varepsilon, \alpha} \mathbf{A}_{Q} v_{\varepsilon, \alpha} \mathrm{d} x$. Consider the decomposition

$$
\begin{aligned}
\int_{\mathbb{R}^{N}} v_{\varepsilon, \alpha} \mathbf{A}_{Q} v_{\varepsilon, \alpha} \mathrm{d} x= & \int_{\mathbb{R}^{N}} v_{\varepsilon} \mathbf{G}_{q} v_{\varepsilon} \mathrm{d} x-\int_{\mathbb{R}^{N}}\left(1+\varphi_{\alpha}\right) v_{\varepsilon} \mathbf{G}_{q}\left(\left(1-\varphi_{\alpha}\right) v_{\varepsilon}\right) \mathrm{d} x \\
& +\int_{\mathbb{R}^{N}} v_{\varepsilon, \alpha}\left(\mathbf{A}_{q}-\mathbf{G}_{q}\right) v_{\varepsilon, \alpha} \mathrm{d} x-\int_{\mathbb{R}^{N}} v_{\varepsilon, \alpha}\left(\mathbf{A}_{q}-\mathbf{A}_{Q}\right) v_{\varepsilon, \alpha} \mathrm{d} x,
\end{aligned}
$$

with $\mathbf{G}_{q}$ as in 2.32 where $Q$ is replaced by the constant function $q$, i.e., $\mathbf{G}_{q}=q^{\frac{1}{2^{*}}} \mathbf{R}_{0} q^{\frac{1}{2^{*}}}$. Starting with the first integral in the right-hand side of $(2.52)$, we remark that 2.50 and (2.51) together with the definition of $\mathbf{G}_{q}$ give

$$
\int_{\mathbb{R}^{N}} v_{\varepsilon} \mathbf{G}_{q} v_{\varepsilon} \mathrm{d} x=q^{\frac{2}{2^{*}}} \int_{\mathbb{R}^{N}} v_{\varepsilon} \mathbf{R}_{0} v_{\varepsilon} \mathrm{d} x=q^{\frac{2}{2^{*}}} S^{\frac{N}{2}} .
$$

Using the Hardy-Littlewood-Sobolev inequality, the second integral in 2.52 can be estimated as follows

$$
\int_{\mathbb{R}^{N}}\left(1+\varphi_{\alpha}\right) v_{\varepsilon} \mathbf{G}_{q}\left(\left(1-\varphi_{\alpha}\right) v_{\varepsilon}\right) \mathrm{d} x \leq q^{\frac{2}{2^{*}}} S^{-1}\left\|\left(1+\varphi_{\alpha}\right) v_{\varepsilon}\right\|_{2^{+}}\left\|\left(1-\varphi_{\alpha}\right) v_{\varepsilon}\right\|_{2^{+}} .
$$

Moreover, since $1-\varphi_{\alpha}=0$ in $B_{\alpha}(0)$, we obtain

$$
\left\|\left(1-\varphi_{\alpha}\right) v_{\varepsilon}\right\|_{2^{+}}^{2^{+}} \leq N \omega_{N}(N(N-2))^{\frac{N}{2}} \int_{\frac{\alpha}{\sqrt{\varepsilon}}}^{\infty} r^{-(N+1)} d r=\omega_{N}(N(N-2))^{\frac{N}{2}} \alpha^{-N} \varepsilon^{\frac{N}{2}} .
$$

Thus, from 2.51 and since $0 \leq \varphi_{\alpha} \leq 1$, it follows that

$$
\int_{\mathbb{R}^{N}}\left(1+\varphi_{\alpha}\right) v_{\varepsilon} \mathbf{G}_{q}\left(\left(1-\varphi_{\alpha}\right) v_{\varepsilon}\right) \mathrm{d} x \leq 2 q^{\frac{2}{2^{*}}}\left(\omega_{N}\right)^{\frac{1}{2^{+}}} S^{\frac{N-2}{4}}(N(N-2))^{\frac{N+2}{4}} \alpha^{-\frac{N+2}{2}} \varepsilon^{\frac{N+2}{4}} .
$$

The third integral in 2.52 can be rewritten as

$$
\begin{aligned}
& \int_{\mathbb{R}^{N}} v_{\varepsilon, \alpha}\left(\mathbf{A}_{q}-\mathbf{G}_{q}\right) v_{\varepsilon, \alpha} \mathrm{d} x \\
& =q^{\frac{2}{2^{*}}} \int_{\mathbb{R}^{N}} v_{\varepsilon, \alpha}\left[(\Psi-\Lambda) * v_{\varepsilon, \alpha}\right] \mathrm{d} x \\
& =q^{\frac{2}{2^{*}}} \int_{\mathbb{R}^{N}} \int_{\mathbb{R}^{N}} v_{\varepsilon}(x) v_{\varepsilon}(y) \varphi_{\alpha}(x) \varphi_{\alpha}(y)[\Psi(x-y)-\Lambda(x-y)] \mathrm{d} y \mathrm{~d} x .
\end{aligned}
$$


Since $\varphi_{\alpha}(x)=0$ for all $|x| \geq 2 \alpha$, it is enough to estimate the difference $\Psi-\Lambda$ inside $B_{4 \alpha}(0)$. Fixing $\alpha_{0} \in\left(0, \frac{1}{4} y_{0}\right)$ and observing that $y_{\nu}<y_{\nu+1}$ for $\nu \geq 0$, we obtain from lemma 2.2 a constant $\kappa_{0}>0$ such that

$$
\Psi(z)-\Lambda(z) \geq\left\{\begin{array}{ll}
\kappa_{0}|z|^{4-N}, & \text { if } N \geq 5, \\
\kappa_{0}|\ln | z||, & \text { if } N=4,
\end{array} \quad \text { for all } 0<|z| \leq 4 \alpha_{0} .\right.
$$

As a consequence, and since $\varphi_{\alpha} \equiv 1$ in $B_{\alpha}$, we can write for all $0<\alpha \leq \alpha_{0}$ and $0<\varepsilon \leq \alpha^{2}$ :

$$
\begin{aligned}
\int_{\mathbb{R}^{N}} v_{\varepsilon, \alpha}\left(\mathbf{A}_{q}-\mathbf{G}_{q}\right) v_{\varepsilon, \alpha} \mathrm{d} x & \geq \kappa_{0} q^{\frac{2}{2^{*}}} \int_{B_{\alpha}} \int_{B_{\alpha}} v_{\varepsilon}(x) v_{\varepsilon}(y)|x-y|^{4-N} \mathrm{~d} y \mathrm{~d} x \\
& =\varepsilon \kappa_{0} q^{\frac{2}{2^{*}}} \int_{B_{\frac{\alpha}{\sqrt{\varepsilon}}}} \int_{\frac{\alpha}{\sqrt{\varepsilon}}} v(x) v(y)|x-y|^{4-N} \mathrm{~d} y \mathrm{~d} x \\
& \geq \varepsilon 2^{4-N} \kappa_{0} q^{\frac{2}{2^{*}}}\left(\int_{B_{1}} v(x) \mathrm{d} x\right)^{2},
\end{aligned}
$$

in the case where $N \geq 5$. In a similar way, we obtain for $N=4$,

$$
\int_{\mathbb{R}^{N}} v_{\varepsilon, \alpha}\left(\mathbf{A}_{q}-\mathbf{G}_{q}\right) v_{\varepsilon, \alpha} \mathrm{d} x \geq \varepsilon|\ln (2 \sqrt{\varepsilon})| \kappa_{0} q^{\frac{2}{2^{*}}}\left(\int_{B_{1}} v(x) \mathrm{d} x\right)^{2} .
$$

Setting $\gamma:=2^{4-N} \kappa_{0} q^{\frac{2}{2^{*}}}\left(\int_{B_{1}} v(x) \mathrm{d} x\right)^{2}$, the above computations yield

$$
\int_{\mathbb{R}^{N}} v_{\varepsilon, \alpha}\left(\mathbf{A}_{q}-\mathbf{G}_{q}\right) v_{\varepsilon, \alpha} \mathrm{d} x \geq \gamma \varepsilon, \quad \text { for all } 0<\alpha \leq \alpha_{0} \text { and } 0<\varepsilon \leq \min \left\{\alpha^{2}, \frac{\mathrm{e}^{-2}}{4}\right\} .
$$

To estimate the remaining integral in 2.52 , we first note that, since $0 \leq \frac{Q(x)}{q} \leq 1$, we have

$$
0 \leq q^{\frac{1}{2^{*}}}-Q^{\frac{1}{2^{*}}}(x) \leq q^{\frac{1}{2^{*}}-1}(q-Q(x)), \quad \text { for all } x .
$$

Thus, the assumption (Q) gives for each $\delta>0$ a constant $\alpha_{\delta}>0$ such that

$$
0 \leq q^{\frac{1}{2^{*}}}-Q^{\frac{1}{2^{*}}}(x) \leq \frac{\delta}{2}|x|^{2}, \quad \text { for all }|x| \leq 2 \alpha_{\delta} .
$$


Since $\varphi_{\varepsilon, \alpha} \equiv 0$ outside $B_{2 \alpha}$, we find for $0<\alpha \leq \alpha_{\delta}$ and $0<\varepsilon \leq \alpha^{2}$,

$$
\begin{aligned}
\int_{\mathbb{R}^{N}} v_{\varepsilon, \alpha}\left(\mathbf{A}_{q}-\mathbf{A}_{Q}\right) v_{\varepsilon, \alpha} \mathrm{d} x & =\int_{\mathbb{R}^{N}}\left(q^{\frac{1}{2^{*}}}-Q^{\frac{1}{2^{*}}}\right) v_{\varepsilon, \alpha} \mathbf{R}\left[\left(q^{\frac{1}{2^{*}}}+Q^{\frac{1}{2^{*}}}\right) v_{\varepsilon, \alpha}\right] \mathrm{d} x \\
& \leq 2 q^{\frac{1}{2^{*}}} C_{0}\left\|\left(q^{\frac{1}{2^{*}}}-Q^{\frac{1}{2^{*}}}\right) v_{\varepsilon, \alpha}\right\|_{2^{+}}\left\|v_{\varepsilon, \alpha}\right\|_{2^{+}} \\
& \leq \delta \varepsilon q^{\frac{1}{2^{*}}} S^{\frac{N+2}{4}} C_{0}\left(\left.\left.\int_{\mathbb{R}^{N}}|| x\right|^{2} v(x)\right|^{2^{+}} \mathrm{d} x\right)^{\frac{1}{2^{+}}}
\end{aligned}
$$

Choosing $\delta>0$ such that $\delta q^{\frac{1}{2^{*}}} S^{\frac{N+2}{4}} C_{0}\left(\left.\left.\int_{\mathbb{R}^{N}}|| x\right|^{2} v(x)\right|^{2^{+}} \mathrm{d} x\right)^{\frac{1}{2^{+}}} \leq \frac{\gamma}{2}$ and setting $\alpha:=\min \left\{\alpha_{0}, \alpha_{\delta}\right\}$, we obtain the estimate

$$
\int_{\mathbb{R}^{N}} v_{\varepsilon, \alpha}\left(\mathbf{A}_{q}-\mathbf{A}_{Q}\right) v_{\varepsilon, \alpha} \mathrm{d} x \leq \frac{\gamma}{2} \varepsilon, \quad \text { for all } 0<\varepsilon \leq \alpha^{2} .
$$

With this choice of $\alpha$, putting the estimates (2.53), 2.54), 2.56) and 2.57) together, the decomposition 2.52 yields

$$
\begin{aligned}
& \int_{\mathbb{R}^{N}} v_{\varepsilon, \alpha} \mathbf{A}_{Q} v_{\varepsilon, \alpha} \mathrm{d} x \geq q^{\frac{2}{2^{*}}} S^{\frac{N}{2}}+\frac{\gamma}{2} \varepsilon-\zeta \varepsilon^{\frac{N+2}{4}} \geq q^{\frac{2}{2^{*}}} S^{\frac{N}{2}}+\frac{\gamma}{4} \varepsilon \\
& >q^{\frac{2}{2^{*}}} S^{\frac{N}{2}}, \quad \text { for } 0<\varepsilon \leq \varepsilon_{0}:=\min \left\{\alpha^{2},\left(\frac{\gamma}{4 \zeta}\right)^{\frac{4}{N-2}}, \frac{\mathrm{e}^{-2}}{4}\right\},
\end{aligned}
$$

where $\zeta=2 q^{\frac{2}{2^{*}}}\left(\omega_{N}\right)^{\frac{1}{2^{*}}} S^{\frac{N-2}{4}}(N(N-2))^{\frac{N+2}{4}} \alpha^{-\frac{N+2}{2}}$. Hence, from (2.34), (2.41), (2.51) and 2.58 we infer that for $\alpha=\min \left\{\alpha_{0}, \alpha_{\delta}\right\}$ and $0<\varepsilon \leq \varepsilon_{0}$,

$$
L_{Q} \leq \frac{1}{N}\left(\frac{\left\|v_{\varepsilon, \alpha}\right\|_{2^{+}}^{2}}{\int_{\mathbb{R}^{N}} v_{\varepsilon, \alpha} \mathbf{A}_{Q} v_{\varepsilon, \alpha} \mathrm{d} x}\right)^{\frac{N}{2}}<\frac{1}{N}\left(\frac{S^{\frac{N+2}{2}}}{q^{\frac{2}{2^{*}} S^{\frac{N}{2}}}}\right)^{\frac{N}{2}}=\frac{S^{\frac{N}{2}}}{N q^{\frac{N-2}{2}}}=L_{Q}^{*} .
$$

This proves the desired result.

REMARK 2.14. In the case $N=4$, using the estimate 2.55) instead of 2.56, we see that the condition $\mathrm{Q}$ can be weakened to

$$
Q\left(x_{0}\right)-Q(x)=O\left(\left|x-x_{0}\right|^{2}\right), \quad \text { as }\left|x-x_{0}\right| \rightarrow 0 .
$$

3.3. Existence and nonexistence of dual ground states. We are now in position to give the proof of our main existence result for the critical nonlinear Helmholtz equation.

Theorem 2.15. Let $N \geq 4$ and consider $Q \in L^{\infty}\left(\mathbb{R}^{N}\right) \backslash\{0\}$. Assume in addition that

(Q1) $Q=Q_{\text {per }}+Q_{0}$, where $Q_{\text {per }}, Q_{0} \geq 0$ are such that $Q_{\text {per }}$ is $\mathbb{Z}^{N}$-periodic and $Q_{0}(x) \rightarrow 0$ as $|x| \rightarrow \infty$; 
(Q2) there exists $x_{0} \in \mathbb{R}^{N}$ with $Q\left(x_{0}\right)=\|Q\|_{\infty}$ and, as $\left|x-x_{0}\right| \rightarrow 0$,

$$
Q\left(x_{0}\right)-Q(x)= \begin{cases}o\left(\left|x-x_{0}\right|^{2}\right), & \text { if } N \geq 5, \\ O\left(\left|x-x_{0}\right|^{2}\right), & \text { if } N=4 .\end{cases}
$$

Then the problem

$$
-\Delta u-u=Q(x)|u|^{2^{*}-2} u, \quad u \in W^{2,2^{*}}\left(\mathbb{R}^{N}\right)
$$

has a dual ground state.

Proof. Using the mountain pass theorem without the Palais-Smale condition (see 4 and [10, theorem 2.2]) we obtain the existence of a Palais-Smale sequence $\left(v_{n}\right)_{n} \subset L^{2^{+}}\left(\mathbb{R}^{N}\right)$ at the mountain pass level $L_{Q}$. Therefore, by proposition 2.11, proposition 2.13 and Remark 2.14 . the functional $J_{Q}$ possesses a critical point $w \in L^{2^{+}}\left(\mathbb{R}^{N}\right)$ of $J_{Q}$ which satisfies $J_{Q}(w)=L_{Q}$. Setting $u=\mathbf{R}\left(Q^{\frac{1}{2^{*}}} w\right)$, we find that $u \in L^{2^{*}}\left(\mathbb{R}^{N}\right)$ is a dual ground state of 2.59), and this concludes the proof.

In dimension $N=3$, the situation completely changes. Indeed, the proof of Proposition 2.13 fails, since the estimate in Lemma 2.2(i) now has the opposite sign. In fact, we have the following nonexistence result.

Proposition 2.16. Let $Q \in L^{\infty}\left(\mathbb{R}^{3}\right) \backslash\{0\}$ satisfy $Q(x) \geq 0$ for almost every $x \in \mathbb{R}^{3}$. Then, there is no dual ground state for the problem

$$
-\Delta u-u=Q(x)|u|^{2^{*}-2} u, \quad u \in W^{2,2^{*}}\left(\mathbb{R}^{3}\right) .
$$

Proof. We start by proving the inequality $L_{Q} \leq L_{Q}^{*}$.

For this, let us consider the family of functions $v_{\varepsilon}, \varepsilon>0$, given in 2.49 . Let $0<\delta<\|Q\|_{\infty}$ be arbitrary but fixed, and consider the set $\omega_{\delta}:=\left\{x \in \mathbb{R}^{3}: Q(x) \geq\|Q\|_{\infty}-\delta\right\}$. Since $\omega_{\delta}$ has positive measure, we can choose a point $x_{\delta} \in \omega_{\delta}$ such that

$$
\lim _{\alpha \rightarrow 0^{+}} \frac{\left|\omega_{\delta} \cap B_{\alpha}\left(x_{\delta}\right)\right|}{\left|B_{\alpha}\left(x_{\delta}\right)\right|}=1,
$$

where $|\cdot|$ denotes the Lebesgue measure. With $\varphi_{\delta}:=\mathbb{1}_{\omega_{\delta} \cap B_{\frac{1}{2}}\left(x_{\delta}\right)}$, we find

$$
\begin{aligned}
& \int_{\mathbb{R}^{3}} \varphi_{\delta} v_{\varepsilon}\left(\cdot-x_{\delta}\right) \mathbf{A}_{Q}\left(\varphi_{\delta} v_{\varepsilon}\left(\cdot-x_{\delta}\right)\right) \mathrm{d} x \\
& =\int_{\mathbb{R}^{3}} \int_{\mathbb{R}^{3}} \varphi_{\delta}(x) \varphi_{\delta}(y) v_{\varepsilon}\left(x-x_{\delta}\right) v_{\varepsilon}\left(y-x_{\delta}\right) Q^{\frac{1}{2^{*}}}(x) Q^{\frac{1}{2^{*}}}(y) \Psi(x-y) \mathrm{d} x \mathrm{~d} y \\
& \geq\left(\|Q\|_{\infty}-\delta\right)^{\frac{2}{2^{*}}} \int_{\mathbb{R}^{3}} \int_{\mathbb{R}^{3}} \varphi_{\delta}(x) \varphi_{\delta}(y) v_{\varepsilon}\left(x-x_{\delta}\right) v_{\varepsilon}\left(y-x_{\delta}\right) \Psi(x-y) \mathrm{d} x \mathrm{~d} y,
\end{aligned}
$$


since $\Psi(x-y)=\frac{\cos |x-y|}{4 \pi|x-y|} \geq 0$ for all $x, y \in B_{\frac{1}{2}}(z), z \in \mathbb{R}^{3}$. Remarking furthermore that $v_{\varepsilon}(x)=\varepsilon^{-\frac{5}{4}} v_{1}\left(\frac{x}{\sqrt{\varepsilon}}\right)$, a change of variables gives

$$
\begin{aligned}
& \int_{\mathbb{R}^{3}} \int_{\mathbb{R}^{3}} \varphi_{\delta}(x) \varphi_{\delta}(y) v_{\varepsilon}\left(x-x_{\delta}\right) v_{\varepsilon}\left(y-x_{\delta}\right) \Psi(x-y) \mathrm{d} x \mathrm{~d} y \\
& =\int_{\mathbb{R}^{3}} \int_{\mathbb{R}^{3}} \varphi_{\delta}\left(\sqrt{\varepsilon} x+x_{\delta}\right) \varphi_{\delta}\left(\sqrt{\varepsilon} y+x_{\delta}\right) v_{1}(x) v_{1}(y) \frac{\cos (\sqrt{\varepsilon}(x-y))}{4 \pi|x-y|} \mathrm{d} x \mathrm{~d} y \\
& \longrightarrow \int_{\mathbb{R}^{3}} \int_{\mathbb{R}^{3}} v_{1}(x) v_{1}(y) \frac{1}{4 \pi|x-y|} \mathrm{d} x \mathrm{~d} y=\int_{\mathbb{R}^{3}} v_{1} \mathbf{R}_{0} v_{1} \mathrm{~d} x, \quad \text { as } \varepsilon \rightarrow 0^{+},
\end{aligned}
$$

using (2.61) in the last step. Thus, we obtain

$$
\begin{aligned}
\liminf _{\varepsilon \rightarrow 0^{+}} \int_{\mathbb{R}^{3}} \varphi_{\delta} v_{\varepsilon}\left(\cdot-x_{\delta}\right) \mathbf{A}_{Q}\left(\varphi_{\delta} v_{\varepsilon}\left(\cdot-x_{\delta}\right)\right) \mathrm{d} x & \geq\left(\|Q\|_{\infty}-\delta\right)^{\frac{2}{2^{*}}} \int_{\mathbb{R}^{3}} v_{1} \mathbf{R}_{0} v_{1} \mathrm{~d} x \\
& =\left(\|Q\|_{\infty}-\delta\right)^{\frac{2}{2^{*}}} S^{-1}\left\|v_{1}\right\|_{2^{+}}^{2} .
\end{aligned}
$$

In addition, since $\left\|\varphi_{\delta} v_{\varepsilon}\left(\cdot-x_{\delta}\right)\right\|_{2^{+}} \rightarrow\left\|v_{1}\right\|_{2^{+}}$, as $\varepsilon \rightarrow 0^{+}$, the characterization 2.34 of $L_{Q}$ yields

$$
L_{Q} \leq \limsup _{\varepsilon \rightarrow 0^{+}} \frac{1}{N}\left(\frac{\left\|\varphi_{\delta} v_{\varepsilon}\left(\cdot-x_{0}\right)\right\|_{2^{+}}^{2}}{\int_{\mathbb{R}^{3}} \varphi_{\delta} v_{\varepsilon}\left(\cdot-x_{0}\right) \mathbf{A}_{Q}\left(\varphi_{\delta} v_{\varepsilon}\left(\cdot-x_{0}\right)\right) \mathrm{d} x}\right)^{\frac{N}{2}} \leq \frac{S^{\frac{N}{2}}}{N\left(\|Q\|_{\infty}-\delta\right)^{\frac{N+2}{2}}} .
$$

Letting now $\delta \rightarrow 0^{+}$, we infer from 2.41) that $L_{Q} \leq L_{Q}^{*}$.

We next assume by contradiction that $L_{Q}$ is achieved. In this case, there exists $v \in L^{2^{+}}\left(\mathbb{R}^{3}\right)$ such that $\|v\|_{2^{+}}=1$ and

$$
\int_{\mathbb{R}^{3}} v \mathbf{A}_{Q} v \mathrm{~d} x=\left(N L_{Q}\right)^{-\frac{2}{N}} .
$$

Since $L_{Q} \leq L_{Q}^{*}$ and recalling the value of $L_{Q}^{*}$ given in 2.41, we can write

$$
\begin{aligned}
& S^{-1}\|Q\|_{\infty}^{\frac{2}{2^{*}}}=\left(N L_{Q}^{*}\right)^{-\frac{2}{N}} \leq\left(N L_{Q}\right)^{-\frac{2}{N}}=\int_{\mathbb{R}^{3}} v \mathbf{A}_{Q} v \mathrm{~d} x \\
& \leq \int_{\mathbb{R}^{3}} Q^{\frac{1}{2^{*}}}|v|\left[|\Psi| *\left(Q^{\frac{1}{2^{*}}}|v|\right)\right] \mathrm{d} x \leq \int_{\mathbb{R}^{3}} Q^{\frac{1}{2^{*}}}|v|\left[\Lambda *\left(Q^{\frac{1}{2^{*}}}|v|\right)\right] \mathrm{d} x \\
& =\int_{\mathbb{R}^{3}} Q^{\frac{1}{2^{*}}}|v| \mathbf{R}_{0}\left(Q^{\frac{1}{2^{*}}}|v|\right) \mathrm{d} x \leq S^{-1}\left\|Q^{\frac{1}{2^{*}}} v\right\|_{2^{+}}^{2} \leq S^{-1}\|Q\|_{\infty}^{\frac{2}{2^{*}}},
\end{aligned}
$$

using the fact that $|\Psi(z)|=\frac{|\cos | z||}{4 \pi|z|} \leq \frac{1}{4 \pi|z|}=\Lambda(z)$ for all $z \in \mathbb{R}^{3}$, and the Hardy-LittlewoodSobolev inequality. As a consequence, all inequalities are equalities and we find $L_{Q}=L_{Q}^{*}$ and 
obtain the following identities.

$$
\begin{gathered}
\int_{\mathbb{R}^{3}} Q^{\frac{1}{2^{*}}}|v| \mathbf{R}_{0}\left(Q^{\frac{1}{2^{*}}}|v|\right) \mathrm{d} x=S^{-1}\left\|Q^{\frac{1}{2^{*}}} v\right\|_{2^{+}}^{2}, \\
\int_{\mathbb{R}^{3}} Q^{\frac{1}{2^{*}}}|v|\left[|\Psi| *\left(Q^{\frac{1}{2^{*}}}|v|\right)\right] \mathrm{d} x=\int_{\mathbb{R}^{3}} Q^{\frac{1}{2^{*}}}|v|\left[\Lambda * \left(Q^{\left.\left.\frac{1}{2^{*}}|v|\right)\right] \mathrm{d} x .}\right.\right.
\end{gathered}
$$

From 2.62 and the uniqueness of the optimizers for the Hardy-Littlewood-Sobolev inequality [46], we deduce that

$$
Q^{\frac{1}{2^{*}}}|v|=\gamma v_{\varepsilon}\left(\cdot-x_{0}\right), \quad \text { for some } \gamma, \varepsilon>0 \text { and } x_{0} \in \mathbb{R}^{3},
$$

where $v_{\varepsilon}$ is given by 2.49 . In particular, $Q^{\frac{1}{2^{*}}}|v|>0$ everywhere in $\mathbb{R}^{3}$, and we obtain

$$
\begin{aligned}
& \int_{\mathbb{R}^{3}} Q^{\frac{1}{2^{*}}}|v|\left[|\Psi| *\left(Q^{\frac{1}{2^{*}}}|v|\right)\right] \mathrm{d} x=\iint_{\mathbb{R}^{3}} \int_{\mathbb{R}^{3}} Q^{\frac{1}{2^{*}}}(x)|v(x)| Q^{\frac{1}{2^{*}}}(y)|v(y)| \frac{|\cos | x-y||}{4 \pi|x-y|} \mathrm{d} x \mathrm{~d} y \\
& <\int_{\mathbb{R}^{3}} \int_{\mathbb{R}^{3}} Q^{\frac{1}{2^{*}}}(x)|v(x)| Q^{\frac{1}{2^{*}}}(y)|v(y)| \frac{1}{4 \pi|x-y|} \mathrm{d} x \mathrm{~d} y \\
& =\int_{\mathbb{R}^{3}} Q^{\frac{1}{2^{*}}}|v|\left[\Lambda *\left(Q^{\frac{1}{2^{*}}}|v|\right)\right] \mathrm{d} x .
\end{aligned}
$$

This contradicts 2.63 and therefore shows that $L_{Q}$ is not achieved. In particular, $J_{Q}$ does not have any critical point at level $L_{Q}$, and thus no dual ground state solution of 2.60 can exist. 



\section{CHAPTER 3}

\section{Fourier-extension estimates and applications to a nonlinear Helmholtz equation}

In the current chapter we will provide our results concerning improved Fourier-extension estimates and their consequences with regard to the nonlinear Helmholtz equation. The presented results follow the presentation of the original article [Y2], where we have excluded the acknowledgement. This article resulted from a collaboration with Tobias Weth. The notation may slightly differ from the notation in the previous chapters.

\section{Introduction}

Starting with the pioneering work of Stein (cf. [60]), Tomas [67] and Strichartz [62], Fourier restriction and extension estimates have been receiving extensive attention due to their various applications, especially to partial differential equations. For an overview on classical results and recent progress, we refer the reader to e.g. [34, 59, 66. In its classical form, the famous Fourier extension theorem of Stein and Tomas (see e.g. [59, §8: Corollary 5.4]) states that the inverse Fourier transform $\check{F}_{\sigma}$ of $F \in L^{2}\left(\mathbb{S}^{N-1}\right)$, given by

$$
\check{F}_{\sigma}(x)=(2 \pi)^{-\frac{N}{2}} \int_{\mathbb{S}^{N-1}} e^{i \omega \cdot x} F(\omega) d \sigma(\omega)
$$

belongs to $L^{q}\left(\mathbb{R}^{N}\right)$ for $N \geq 2$ if $q \geq \frac{2(N+1)}{N-1}$, and that

$$
\left\|\check{F}_{\sigma}\right\|_{L^{q}\left(\mathbb{R}^{N}\right)} \leq C\|F\|_{L^{2}\left(\mathbb{S}^{N-1}\right)}
$$

with a constant $C>0$ depending only on $q$ and $N$. Here $\mathbb{S}^{N-1}$ denotes the $(N-1)$-dimensional sphere in $\mathbb{R}^{N}$ and $d \sigma$ the induced Lebesgue measure on $\mathbb{S}^{N-1}$. Due to the Knapp example given by a characteristic function of a small spherical cap in $\mathbb{S}^{N-1}$, this range of exponents is known to be sharp for arbitrary functions, see e.g [66, Chapter 4]. On the other hand, it is a natural question whether the range of exponents can be improved both by considering weighted $L^{q}$-norms and by restricting to functions having additional symmetries. A well known and classical observation in this context yields that case (3.1) holds for $q>\frac{2 N}{N-1}$ and radial (and thus constant) functions $F \in L^{2}\left(\mathbb{S}^{N-1}\right)$, see e.g. [59, §8: Proposition 5.1].

In the present paper, we analyze this question for more general symmetries with respect to closed subgroups of $O(N)$.

For this we introduce the following definition. 
Definition 3.1. Let $q \geq 1$, let $G \subset O(N)$ be closed subgroup, and let $Q: \mathbb{R}^{N} \rightarrow \mathbb{C}$ be a measurable function. We call $(G, q, Q)$ an admissible extension triple if there exists a constant $C>0$ with

$$
\left\|Q \check{F}_{\sigma}\right\|_{L^{q}\left(\mathbb{R}^{N}\right)} \leq C\|F\|_{L^{2}\left(\mathbb{S}^{N-1}\right)} \quad \text { for every } G \text {-invariant function } F \in L^{2}\left(\mathbb{S}^{N-1}\right) .
$$

Here and in the following, a function $F \in L^{2}\left(\mathbb{S}^{N-1}\right)$ is called $G$-invariant if $F(A \theta)=F(\theta)$ for every $\theta \in \mathbb{S}^{N-1}, A \in G$. By the remarks above, $(\{\mathrm{id}\}, q, 1)$ is an admissible extension triple if $q \geq \frac{2(N+1)}{N-1}$ and $(O(N), q, 1)$ is an admissible extension triple if $q>\frac{2 N}{N-1}$. As a further specific example, we mention the subgroup $O(N-1) \cong O(N-1) \times\left\{\operatorname{id}_{\mathbb{R}}\right\} \subset O(N)$ which corresponds to axial symmetry with respect to a fixed axis in $\mathbb{R}^{N}$. Since a characteristic function of a small spherical cap in $\mathbb{S}^{N-1}$ - as considered in Knapp's example mentioned above - is axially symmetric, the range for $q$ with $(O(N-1), q, 1)$ being an admissible extension triple cannot be extended beyond the value $\frac{2(N+1)}{N-1}$.

If, on the other hand, we consider weight functions $Q \in L^{s}\left(\mathbb{R}^{N}\right)$ for suitable $s<\infty$, then the range of exponents giving rise to admissible extension triples can be readily extended by applying Hölder's inequality to the LHS of $(3.2)$. In particular, this yields that $(\{\mathrm{id}\}, q, Q)$ is an admissible extension triple if $Q \in L^{s}\left(\mathbb{R}^{N}\right)$ for some $s \in[1, \infty)$ and $q \geq \max \left\{\frac{2 s(N+1)}{2(N+1)+s(N-1)}, 1\right\}$. Moreover, $(O(N), q, Q)$ is an admissible extension triple if $Q \in L^{s}\left(\mathbb{R}^{N}\right)$ for some $s \in[1, \infty)$ and $q \geq \max \left\{\frac{2 s N}{2 N+s(N-1)}, 1\right\}$.

In the present paper, we are interested in weight functions $Q \in L^{\infty}\left(\mathbb{R}^{N}\right)$, where Hölder's inequality does not yield an extended range of admissible exponents. The main aims of the paper are the following. First, we wish to detect a class of admissible extension triples corresponding to nontrivial subgroups of $O(N)$ and corresponding to functions $Q \in L^{\infty}\left(\mathbb{R}^{N}\right)$ which are not $s$-integrable for any $s<\infty$. Second, starting from a range of admissible extension triples $(G, q, Q)$, we wish to derive selfdual $\left(L^{p^{\prime}}, L^{p}\right)$-estimates for the restriction of mappings of the form

$$
f \mapsto \mathcal{R}_{Q} f:=Q \mathcal{R}(Q f)
$$

to $G$-invariant functions in the Schwartz space $\mathcal{S}$ of rapidly decreasing functions in $\mathbb{R}^{N}$. Here $\mathcal{R}$ denotes the standard Helmholtz resolvent defined by $\mathcal{R} f=\Phi * f$, where

$$
\Phi(x):=\frac{\mathrm{i}}{4}(2 \pi|x|)^{\frac{2-N}{2}} H_{\frac{N-2}{2}}^{(1)}(|x|), \quad \text { for } x \in \mathbb{R}^{N} \backslash\{0\},
$$

is the fundamental solution of the Helmholtz operator associated with Sommerfeld's outgoing radiation condition $\partial_{r} u(x)-\mathrm{i} u(x)=o\left(|x|^{\frac{1-N}{2}}\right)$, as $|x| \rightarrow \infty$. Here $H_{\frac{N-2}{2}}^{(1)}$ denotes the Hankel function of the first kind of order $\frac{N-2}{2}$. Moreover, we wish to derive corresponding nonvanishing results in the spirit of [31, Theorem 3.1]. Finally, we wish to deduce existence results for realvalued $G$-invariant solutions of nonlinear Helmholtz equations of the form

$$
-\Delta u-u=Q(x)|u|^{p-2} u, \quad u \in W^{2, p}\left(\mathbb{R}^{N}\right) .
$$


With regard to our first aim, we focus our attention to the subgroups

$$
G_{k}:=O(N-k) \times O(k) \subset O(N) \quad \text { for } k=1, \ldots, N-1 .
$$

Moreover, we consider weight functions of the form $Q_{\alpha}=\mathbb{1}_{L_{\alpha}}$ for the set

$$
L_{\alpha}:=\left\{x=\left(x^{(N-k)}, x^{(k)}\right) \in \mathbb{R}^{N-k} \times \mathbb{R}^{k}:\left|x^{(N-k)}\right| \leq a\left|x^{(k)}\right|^{-\alpha}\right\},
$$

where $a>0$ is an arbitrary fixed number and $\alpha>0$. Since $\left|L_{\alpha}\right|=\infty$, we have $Q_{\alpha} \notin L^{s}\left(\mathbb{R}^{N}\right)$ for any $\alpha>0, s<\infty$.

Theorem 3.2. Let $N \geq 3, k \in\{1, \ldots, N-1\}$, let $\alpha>0$, and let $Q_{\alpha}=\mathbb{1}_{L_{\alpha}}$ with $L_{\alpha}$ given in (3.6). Moreover, suppose that

$$
\alpha>\frac{1}{N-1} \quad \text { if } k=1, \quad \alpha<N-1 \quad \text { if } k=N-1,
$$

and let

$$
\lambda_{N, k, \alpha}:= \begin{cases}\frac{2(N-1)-\frac{2}{\alpha}}{N-2}, & \text { if } k=1 ; \\ \max \left\{\frac{2(N-k)-\frac{2 k}{\alpha}}{N-k-1}, \frac{2 k-2 \alpha(N-k)}{k-1}\right\} & \text { if } 2 \leq k \leq N-2 ; \\ \frac{2(N-1)-2 \alpha}{N-2}, & \text { if } k=N-1 .\end{cases}
$$

Then $\left(G_{k}, q, Q_{\alpha}\right)$ is an admissible extension triple for every $q>\lambda_{N, k, \alpha}$.

We note that, in Theorem 3.10 below, we shall in fact prove a generalization of this result for characteristic functions of sets of the form $L_{\alpha, \beta}:=\left\{x \in \mathbb{R}^{N}:\left|x^{(N-k)}\right| \leq a \max \left\{\left|x^{(k)}\right|^{-\alpha},\left|x^{(k)}\right|^{-\beta}\right\}\right\}$ with $\alpha \geq \beta>0$. Regarding Theorem 3.2 , we note in particular that $\lambda_{N, k, \alpha}=0$ for $\alpha=\frac{k}{N-k}$, so $\left(G_{k}, q, Q_{\alpha}\right)$ is an admissible extension triple for every $q \geq 1$ in this case if also (3.7) is satisfied. More generally, the latter property holds if $\alpha \in\left(\frac{k+1}{2(N-k)}, \frac{2 k}{N-k+1}\right)$, since then we have $\lambda_{N, k, \alpha}<1$. Comparing with the classical Stein-Tomas exponent, we have $\lambda_{N, k, \alpha}<\frac{2(N+1)}{N-1}$ if

$$
\begin{array}{ll}
k \leq \frac{N-1}{2}, & \alpha \in\left(\frac{N+1-2 k}{(N-k)(N-1)}, \infty\right) \quad \text { or } \\
\frac{N-1}{2}<k<\frac{N+1}{2}, & \alpha \in\left(\frac{N+1-2 k}{(N-k)(N-1)}, \frac{k(N-1)}{2 k-(N-1)}\right) \\
k \geq \frac{N+1}{2}, & \alpha \in\left(0, \frac{k(N-1)}{2 k-(N-1)}\right) .
\end{array}
$$

The main part of the proof of this Theorem consists in a detailed asymptotic study of onedimensional integrals which arise after integrating along the orbits of $G_{k}$. Here, the well-known bound

$$
\left|d \check{\sigma}_{k}(x)\right| \leq C(1+|x|)^{\frac{1-k}{2}}, \quad x \in \mathbb{R}^{k}
$$




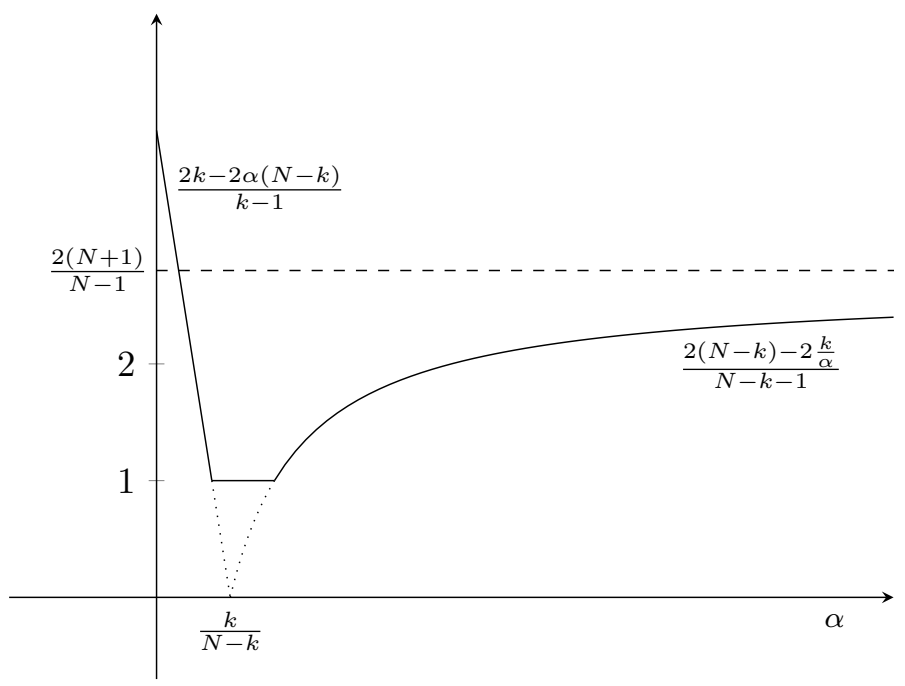

Figure 1. Possible values of $q$ for $N=6$ and $k=2$ depending on $\alpha$.

for the Fourier transform of the standard measure $d \sigma_{k}$ on $\mathbb{S}^{k-1}$ will play a key role (see e.g. [59, §8: Theorem 3.1]).

We also remark that, if $(G, q, Q)$ is an admissible extension triple and $Q^{\prime}: \mathbb{R}^{N} \rightarrow \mathbb{C}$ is a measurable function with $\left|Q^{\prime}\right| \leq|Q|$ in $\mathbb{R}^{N}$, then, by definition, $\left(G, q, Q^{\prime}\right)$ is also an admissible extension triple. Consequently, the statement of Theorem 3.2 extends to functions $Q \in L^{\infty}\left(\mathbb{R}^{N}\right)$ with $|Q| \leq c \mathbb{1}_{L_{\alpha}}$ in $\mathbb{R}^{N}$ for some $c>0$.

Next we state our main result on $\left(L^{p^{\prime}}, L^{p}\right)$-Helmholtz resolvent estimates for $G$-invariant functions. Here and in the following, for $r \in[1, \infty]$, we let $L_{G}^{r}\left(\mathbb{R}^{N}\right)$ denotes the closed subspace of $G$-invariant functions in $L^{r}\left(\mathbb{R}^{N}\right)$.

Theorem 3.3. Let $N \geq 3$, let $G \subset O(N)$ be a closed subgroup, let $Q \in L_{G}^{\infty}\left(\mathbb{R}^{N}\right)$, and let $q \in\left[1, \frac{2(N+1)}{N-1}\right]$ be such that $(G, q, Q)$ is an admissible extension triple. Then for every $p \in$ $\left(\frac{2 N}{N-1} \frac{2 q}{q+2}, \frac{2 N}{N-2}\right]$ there exists a constant $C>0$ such that

$$
\left\|\mathcal{R}_{Q}(f)\right\|_{L^{p}} \leq C\|f\|_{L^{p^{\prime}}} \quad \text { for every } f \in \mathcal{S}_{G}
$$

Here and in the following, $\mathcal{S}_{G} \subset \mathcal{S}$ denotes the subspace of $G$-invariant functions in the Schwartz space $\mathcal{S}$.

Our proof of Theorem 3.3 is based on the strategy used in [37] and [43, see also [31]. We recall that a selfdual estimate of the form (3.9) has been proved in 43 . for the Helmholtz resolvent $\mathcal{R}$ in place of $\mathcal{R}_{Q}$ in the range of exponents $p \in\left[\frac{2(N+1)}{N-1}, \frac{2 N}{N-2}\right]$, while corresponding non-selfdual estimates were obtained in [37. Clearly, these already available $\left(L^{r}, L^{s}\right)$-estimates for $\mathcal{R}$ extend, by approximation, to the weighted resolvent $\mathcal{R}_{Q}$ in the case where $Q \in L^{\infty}\left(\mathbb{R}^{N}\right)$. Theorem 3.3 complements the selfdual estimate in [43], for $\mathcal{R}_{Q}$ and $G$-invariant functions, in the case where $(G, q, Q)$ is an admissible extension triple for some $q<\frac{2(N+1)}{N-1}$, which is equivalent 
to the inequality $\frac{2 N}{N-1} \frac{2 q}{q+2}<\frac{2(N+1)}{N-1}$. In fact, we will prove a non-selfdual generalization of Theorem 3.3 in Theorem 3.12 below.

Under the assumptions of Theorem 3.3 , it follows, by density, that the weighted resolvent $\mathcal{R}_{Q}$ extends to a bounded linear operator $L_{G}^{p^{\prime}}\left(\mathbb{R}^{N}\right) \rightarrow L_{G}^{p}\left(\mathbb{R}^{N}\right)$. In our next result we state that, under the same assumptions, a nonvanishing property in the spirit of [31, Theorem 3.1] holds.

Theorem 3.4. Let $N \geq 3$, let $G \subset O(N)$ be a closed subgroup, let $Q \in L_{G}^{\infty}\left(\mathbb{R}^{N}\right)$, and let $q \in\left[1, \frac{2(N+1)}{N-1}\right]$ be such that $(G, q, Q)$ is an admissible extension triple. Moreover, let $p \in$ $\left(\frac{2 N}{N-1} \frac{2 q}{q+2}, \frac{2 N}{N-2}\right]$. Then for every bounded sequence $\left(v_{n}\right)_{n} \subset L_{G}^{p^{\prime}}\left(\mathbb{R}^{N}\right)$ satisfying

$$
\left|\limsup _{n \rightarrow \infty} \int_{\mathbb{R}^{N}} v_{n} \mathcal{R}_{Q}\left(v_{n}\right) d x\right|>0,
$$

there exist - after passing to a subsequence - numbers $R, \zeta>0$ and a sequence of points $\left(x_{n}\right)_{n \in \mathbb{N}} \subset \mathbb{R}^{N}$ with

$$
\int_{B_{R\left(x_{n}\right)}}\left|Q v_{n}(x)\right|^{p^{\prime}} d x \geq \zeta, \quad \text { for all } n .
$$

In the special (non-symmetric) case $G=\{\operatorname{id}\}, Q \equiv 1, q=\frac{2(N+1)}{N-1}$, this theorem reduces to [31, Theorem 3.1]. Here we note that $\frac{2 N}{N-1} \frac{2 q}{q+2}=\frac{2(N+1)}{N-1}$ if $q=\frac{2(N+1)}{N-1}$. The general strategy of the proof of Theorem 3.4 is inspired by [31, Theorem 3.1]. However, additional difficulties, related to the fact that the multiplication with $Q \in L^{\infty}\left(\mathbb{R}^{N}\right)$ does not map $\mathcal{S}$ into itself, lead to a somewhat more involved argument.

Theorems 3.3 and 3.4 are useful in the study of real-valued $G$-invariant solutions of the nonlinear Helmholtz equation (3.4) with a real-valued weight function $Q \in L_{G}^{\infty}\left(\mathbb{R}^{N}\right)$, where $G \subset O(N)$ is a given closed subgroup. In the following, we focus on dual bound state solutions, which arise as solutions $u \in L^{p}\left(\mathbb{R}^{N}\right)$ of the integral equation $u=R\left(Q|u|^{p-2} u\right)$, where $R$ is the real part of the resolvent operator $\mathcal{R}$, see Section 5 for details. Our first main result in this context is the following.

Theorem 3.5. Let $N \geq 3$, let $G \subset O(N)$ be a closed subgroup, and let $Q \in L_{G}^{\infty}\left(\mathbb{R}^{N}\right)$ be a real-valued nonnegative function with $Q \not \equiv 0$ and with the property that

$$
\|Q\|_{L^{1}\left(B_{R}(x)\right)} \rightarrow 0 \quad \text { as }|x| \rightarrow \infty \text { for some } R>0 .
$$

Moreover, let $q \in\left[1, \frac{2(N+1)}{N-1}\right]$, and let $p \in\left(\max \left\{\frac{2 N}{N-1} \frac{2 q}{q+2}, 2\right\}, \frac{2 N}{N-2}\right)$ be such that $\left(G, q, Q^{\frac{1}{p}}\right)$ is an admissible extension triple. Then (3.4) admits a nontrivial $G$-invariant dual bound state solution.

We recall that, by the Stein-Tomas inequality, $\left(G, q, Q^{\prime}\right)$ is an admissible extension triple for $q=\frac{2(N+1)}{N-1}$ and every closed subgroup $G \subset O(N)$ and every $Q^{\prime} \in L^{\infty}\left(\mathbb{R}^{N}\right)$. Recalling moreover that $\frac{2 N}{N-1} \frac{2 q}{q+2}=\frac{2(N+1)}{N-1}$ in this case, we readily deduce the following corollary of Theorem 3.5 . 
Corollary 3.6. Let $N \geq 3$, let $G \subset O(N)$ be a closed subgroup, and let $Q \in L_{G}^{\infty}\left(\mathbb{R}^{N}\right)$ be a nonnegative function satisfying $Q \not \equiv 0$ and $(3.10)$. Then (3.4) admits a nontrivial $G$-invariant dual bound state solution for every $p \in\left(\frac{2(N+1)}{N-1}, \frac{2 N}{N-2}\right)$.

This corollary applies in particular in the non-symmetric case $G=\{\operatorname{id}\}$, but it requires the asymptotic condition (3.10). On the other hand, in the case of special symmetries corresponding to the subgroups $G_{k}$ defined in (3.5), we may drop assumption (3.10), as the following result shows.

Theorem 3.7. Let $N \geq 4$, let $k \in\{2, \ldots, N-2\}$, and let $Q \in L_{G_{k}}^{\infty}\left(\mathbb{R}^{N}\right)$ be a nonnegative function with $Q \not \equiv 0$. Then (3.4) admits a nontrivial $G_{k}$-invariant dual bound state solution for every $p \in\left(\frac{2(N+1)}{N-1}, \frac{2 N}{N-2}\right)$.

Finally, we point out that assumption 3.10 holds in particular for functions $Q \in L^{\infty}\left(\mathbb{R}^{N}\right)$ satisfying $|Q| \leq c \mathbb{1}_{L_{\alpha}}$ for some $c, \alpha>0$, where $L_{\alpha}$ is given in (3.6). Using this fact, the following corollary can be deduced from Theorems 3.2 and 3.5 .

Corollary 3.8. Let $N \geq 3$, let $k \in\{1, \ldots, N-1\}$, and let $\alpha>0$. Moreover, let $Q \in L_{G_{k}}^{\infty}\left(\mathbb{R}^{N}\right)$ be a nonnegative function with $Q \not \equiv 0$ and satisfying $|Q| \leq c \mathbb{1}_{L_{\alpha}}$ for some $c>0$ with $L_{\alpha}$ given in (3.6). Then (3.4) admits a nontrivial $G_{k}$-invariant dual bound state solution for all $p \in\left(\mu_{N, k, \alpha}, \frac{2 N}{N-2}\right)$ if one of the following holds:

(i) $k=1$ and

$$
\mu_{N, 1, \alpha}:= \begin{cases}2, & \frac{1}{N-1}<\alpha \leq \frac{N+1}{3(N-1)}, \\ \frac{4 N(\alpha(N-1)-1)}{(N-1)(2 \alpha N-3 \alpha-1)}, & \alpha>\frac{N+1}{3(N-1)} .\end{cases}
$$

(ii) $k=N-1$ and

$$
\mu_{N, N-1, \alpha}:= \begin{cases}\frac{4 N(N-1-\alpha)}{(N-1)(2 N-\alpha-3)}, & 0<\alpha \leq \frac{3(N-1)}{N+1}, \\ 2, & \frac{3(N-1)}{N+1}<\alpha<N-1 .\end{cases}
$$

(iii) $2 \leq k \leq N-2$ and

$$
\mu_{N, k, \alpha}:= \begin{cases}\frac{4 N(k-\alpha(N-k))}{(N-1)(2 k-1-\alpha(N-k))}, & \alpha \leq \frac{N+2 k-1}{(N+1)(N-k)}, \\ 2, & \frac{N+2 k-1}{(N+1)(N-k)}<\alpha \leq \frac{(N+1) k}{N-1+2(N-k)}, \\ \frac{4 N(\alpha(N-k)-k)}{(N-1)(2 \alpha(N-k)-\alpha-k)}, & \alpha>\frac{(N+1) k}{N-1+2(N-k)} .\end{cases}
$$

We point out that, in contrast to Corollary 3.6 and Theorem 3.7, Corollary 3.8 allows to consider exponents $p<\frac{2(N+1)}{N-1}$.

To put our existence results for (3.4) into perspective, we recall some previous results. In [37, the existence of small complex solutions has been proved via the use of contraction mappings in dimensions $N=3,4, p=3$ and $Q= \pm 1$. A variant of this technique is developed 
in [51], where continua of small real-valued solutions of (3.4) are detected for a larger class of nonlinearities. The dual variational approach to (3.4) was introduced in [31, where the existence of nontrivial dual bound state solutions was proved for $p \in\left(\frac{2(N+1)}{N-1}, \frac{2 N}{N-2}\right)$ and for nonnegative weight functions $Q \in L^{\infty}\left(\mathbb{R}^{N}\right) \backslash\{0\}$ which are either $\mathbb{Z}^{N}$-periodic or satisfy the uniform decay assumptions $Q(x) \rightarrow 0$ as $|x| \rightarrow \infty$. Under additional restrictions on $Q$, this approach was extended to the Sobolev critical case $p=\frac{2 N}{N-2}$ in [Y1]. Moreover, a dual approach in Orlicz spaces was developed in [27] to treat more general nonlinearities in (3.4). The defocusing case $Q \leq 0$ in (3.4) and radial solutions are considered in [52]. We are not aware of any previous work where symmetries different from radial symmetry are used to extend the range of admissible exponents to values below the Stein-Tomas exponent $\frac{2(N+1)}{N-1}$ and to overcome lack of compactness issues in the context of (3.4). .

The paper is organized as follows. In Section 2, we derive a Fourier extension estimate for $G_{k}$-invariant functions, where $G_{k}$ is defined in 3.5. In particular, we prove a generalization of Theorem 3.2. In Section 3, we provide weighted Helmholtz resolvent estimates relative to a given admissible extension triple, thereby giving the proof of Theorem 3.3 . In Section 4 we study related nonvanishing properties, and we give the proof of Theorem 3.4. Finally, Section 5 is devoted to our main existence results for dual bound state solutions of (3.4).

We close this introduction by fixing some notation. Throughout the paper we denote by $B_{r}(x)$ the open ball in $\mathbb{R}^{N}$ with radius $r>0$ and center at $x$. Moreover, we set $B_{r}=B_{r}(0)$ and $\mathbb{S}^{N-1}$ for the boundary of $B_{1}=: B$. The constant $\alpha_{N}$ represents the volume of the unit ball $B_{1}$ in $\mathbb{R}^{N}$. For any element $x \in \mathbb{R}^{N}$ we write $x=\left(x^{(N-k)}, x^{(k)}\right):=\left(\left(x_{1}, \ldots, x_{N-k}\right),\left(x_{N_{k}+1}, \ldots, x_{N}\right)\right) \in$ $\mathbb{R}^{N-k} \times \mathbb{R}^{k}$. Moreover by $B^{(k)}$ we denote the unit ball in $\mathbb{R}^{k}$. By $\mathbb{1}_{L}$ we denote the characteristic function of a measurable set $L \subset \mathbb{R}^{N}$. Furthermore, we shall indifferently denote by $\widehat{f}$ or $\mathcal{F}(f)$ the Fourier transform of a function in $\mathbb{R}^{N}$ given by

$$
\mathcal{F} f(\xi)=\widehat{f}(\xi)=(2 \pi)^{-\frac{N}{2}} \int_{\mathbb{R}^{N}} e^{-i x \cdot \xi} f(x) d x
$$

and by $\check{F}_{\sigma}$ the inverse Fourier transform of an admissible functions $F$ defined on $\mathbb{S}^{N-1}$ via

$$
\check{F}_{\sigma}(x)=(2 \pi)^{-\frac{N}{2}} \int_{\mathbb{S}^{N-1}} e^{i x \cdot \omega} F(\omega) d \sigma(\omega) .
$$

For $1 \leq s \leq \infty$, we abbreviate the norm on $L^{s}\left(\mathbb{R}^{N}\right)$ by $\|\cdot\|_{s}$. The Schwartz-class of rapidly decreasing functions on $\mathbb{R}^{N}$ is denoted by $\mathcal{S}$. For any $p \in(1, \infty)$ we always denote by $p^{\prime}:=\frac{p}{p-1}$ the Hölder conjugate of $p$. 


\section{Fourier extension estimates for $G_{k}$-invariant functions}

We recall that, for a function $F \in L^{2}\left(\mathbb{S}^{N-1}\right)$, we define the (inverse) Fourier transform of $F d \sigma$ by

$$
\check{F}_{\sigma}(x)=(2 \pi)^{-\frac{N}{2}} \int_{\mathbb{S}^{N-1}} e^{i \omega \cdot x} F(\omega) d \sigma(\omega) .
$$

For $F \equiv 1$ we use the notation

$$
\check{d \sigma_{N}}(x)=(2 \pi)^{-\frac{N}{2}} \int_{\mathbb{S}^{N-1}} e^{i \omega \cdot x} d \sigma(\omega)
$$

and will often omit the dimensional index if no confusion is possible. We point out that this function satisfies the key uniform bound

$$
\left|\check{d \sigma_{N}}(x)\right| \leq C(1+|x|)^{\frac{1-N}{2}}, \quad x \in \mathbb{R}^{N} .
$$

with a constant $C=C(N)>0$, see e.g. [59, §8: Theorem 3.1].

For $k \in\{1, \ldots, N-1\}$, we consider the closed subgroup $G_{k}=O(N-k) \times O(k) \subset O(N)$. We first derive a useful expression for $\check{F}_{\sigma}$ in the case where $F \in \mathcal{C}\left(\mathbb{S}^{N-1}\right)$ is $G_{k^{-}}$-invariant. Note that in this case $F$ only depends on one variable $r \in[0,1]$ via the function

$$
h_{F}:[0,1] \rightarrow \mathbb{R}, \quad h_{F}(r):=F\left(r \eta, \sqrt{1-r^{2}} \mu\right) \quad \text { for } \eta \in \mathbb{S}^{N-k-1}, \mu \in \mathbb{S}^{k-1} .
$$

Lemma 3.9. Let $k \in\{1, \ldots, N-1\}$ and $F \in \mathcal{C}\left(\mathbb{S}^{N-1}\right)$ be $G_{k}$-invariant. Then we have

$$
\check{F}_{\sigma}(x)=(2 \pi)^{\frac{N}{2}} \frac{k \alpha_{k}}{N \alpha_{N}} \int_{0}^{1} r^{N-k-1}\left(1-r^{2}\right)^{\frac{k-2}{2}} h_{F}(r) \check{d \sigma_{N-k}}\left(r x^{(N-k)}\right) \check{d \sigma} \sigma_{k}\left(\sqrt{1-r^{2}} x^{(k)}\right) d r
$$

with $h_{F}$ given in 3.15). Moreover,

$\left|\check{F}_{\sigma}(x)\right| \leq(2 \pi)^{\frac{N}{2}} \frac{k \alpha_{k}}{N \alpha_{N}} \frac{\|F\|_{L^{2}\left(\mathbb{S}^{N-1}\right)}}{\sqrt{\left|\mathbb{S}^{N-k-1}\right|\left|\mathbb{S}^{k-1}\right|}}\left(\int_{0}^{1} r^{N-k-1}\left(1-r^{2}\right)^{\frac{k-2}{2}}\left|\check{d \sigma} \sigma_{N-k}\left(r x^{(N-k)}\right)\right|^{2}\left|\check{d \sigma} \sigma_{k}\left(\sqrt{1-r^{2}} x^{(k)}\right)\right|^{2}\right)^{\frac{1}{2}}$

for all $x \in \mathbb{R}^{N}$. 
Proof. By using slice integration (see e.g. [6, A.5]) we have

$$
\begin{aligned}
& \check{F}_{\sigma}(x)=(2 \pi)^{\frac{N}{2}} \int_{\mathbb{S}^{N-1}} e^{i x \cdot \omega} F(\omega) d \sigma(\omega) \\
& =(2 \pi)^{\frac{N}{2}} \frac{k \alpha_{k}}{N \alpha_{N}} \int_{B^{(N-k)}}\left(1-|y|^{2}\right)^{\frac{k-2}{2}} e^{i x^{(N-k)} y} \int_{\mathbb{S}^{k-1}} e^{i x^{(k)} \sqrt{1-|y|^{2}} \mu} F\left(y, \sqrt{1-|y|^{2}} \mu\right) d \sigma_{k}(\mu) d_{N-k}(y) \\
& =(2 \pi)^{\frac{N}{2}} \frac{k \alpha_{k}}{N \alpha_{N}} \int_{0}^{1} r^{N-k-1}\left(1-r^{2}\right)^{\frac{k-2}{2}} \int_{\mathbb{S}^{N-k-1}} e^{i x^{(N-k)} r \eta} \int_{\mathbb{S}^{k-1}} e^{i x^{(k)} \sqrt{1-r^{2}} \mu} F\left(r \eta, \sqrt{1-r^{2}} \mu\right) d \sigma_{k}(\mu) d \sigma_{N-k}(\eta) d r \\
& =(2 \pi)^{\frac{N}{2}} \frac{k \alpha_{k}}{N \alpha_{N}} \int_{0}^{1} r^{N-k-1}\left(1-r^{2}\right)^{\frac{k-2}{2}} h_{F}(r) \check{d \sigma_{N-k}}\left(r x^{(N-k)}\right) \check{d \sigma_{k}}\left(\sqrt{1-r^{2}} x^{(k)}\right) d r
\end{aligned}
$$

for all $x \in \mathbb{R}^{N}$ with $h_{F}$ given in $(3.15)$, as claimed in $(3.16)$. In particular, we get

$$
\begin{aligned}
& \left|\check{F}_{\sigma}(x)\right| \leq(2 \pi)^{\frac{N}{2}} \frac{k \alpha_{k}}{N \alpha_{N}} \int_{0}^{1} r^{N-k-1}\left(1-r^{2}\right)^{\frac{k-2}{2}}\left|h_{F}(r)\right|\left|\check{d \sigma} \sigma_{N-k}\left(r x^{(N-k)}\right)\right|\left|\check{d \sigma} \sigma_{k}\left(\sqrt{1-r^{2}} x^{(k)}\right)\right| d r . \\
& \leq(2 \pi)^{\frac{N}{2}} \frac{k \alpha_{k}}{N \alpha_{N}}\left(\int_{0}^{1} r^{N-k-1}\left(1-r^{2}\right)^{\frac{1}{2}}\left|h_{F}(r)\right|^{2} d r\right)^{\frac{k-2}{2}} \times \\
& \quad\left(\int_{0}^{1} r^{N-k-1}\left(1-r^{2}\right)^{\frac{k-2}{2}}\left|\check{d \sigma}{ }_{N-k}\left(r x^{(N-k)}\right)\right|^{2}\left|\check{d \sigma}\left(\sqrt{1-r^{2}} x^{(k)}\right)\right|^{2} d r\right)^{\frac{1}{2}} \\
& =(2 \pi)^{\frac{N}{2}} \frac{k \alpha_{k}}{N \alpha_{N}} \frac{\|F\|_{L^{2}\left(\mathbb{S}^{N-1}\right)}}{\sqrt{\left|\mathbb{S}^{N-k-1}\right|\left|\mathbb{S}^{k-1}\right|}}\left(\int_{0}^{1} r^{N-k-1}\left(1-r^{2}\right)^{\frac{k-2}{2}}\left|\check{d \sigma} \sigma_{N-k}\left(r x^{(N-k)}\right)\right|^{2}\left|\check{d \sigma} \sigma_{k}\left(\sqrt{1-r^{2}} x^{(k)}\right)\right|^{2} d r\right)^{\frac{1}{2}} .
\end{aligned}
$$

For $\alpha \geq \beta>0$ and fixed $a>0$, we now consider the subset

$$
L_{\alpha, \beta}:=\left\{x=\left(x^{(N-k)}, x^{(k)}\right):\left|x^{(N-k)}\right| \leq a \max \left\{\left|x^{(k)}\right|^{-\alpha},\left|x^{(k)}\right|^{-\beta}\right\}\right\} \subset \mathbb{R}^{N} .
$$

We shall prove the following generalization of Theorem 3.2 .

Theorem 3.10. Let $N \geq 3$. Suppose we have $\alpha \geq \beta>0, k \in\{1, \ldots, N-1\}, Q=\mathbb{1}_{L_{\alpha, \beta}}$ with $L_{\alpha, \beta}$ given as in (3.6), and

$$
\lambda_{N, k, \alpha, \beta}:=\max \left\{\frac{2 k-2 \beta(N-k)}{k-1}, \frac{2(N-k)-\frac{2 k}{\alpha}}{N-k-1}\right\} .
$$


Suppose furthermore that $q \geq 1$ and $k \in\{1, \ldots, N-1\}$ satisfy

$$
\left\{\begin{array}{lll}
k=1, & \beta>\frac{1}{N-1} & \text { and } q>\frac{2(N-1)-\frac{2}{\alpha}}{N-2} \text { or } \\
2 \leq k \leq N-2, & q>\lambda_{N, k, \alpha, \beta} \quad \text { or } \\
k=N-1, & \alpha<N-1, \quad \text { and } q>\frac{2(N-1)-2 \beta}{N-2} .
\end{array}\right.
$$

Then there exists a constant $C=C(N, k, \alpha, \beta, a)$ with the property that

$$
\left\|Q \check{F}_{\sigma}\right\|_{q} \leq C\|F\|_{L^{2}\left(\mathbb{S}^{N-1}\right)} \quad \text { for every } G_{k} \text {-invariant function } F \in \mathcal{C}\left(\mathbb{S}^{N-1}\right) .
$$

Proof. We shall prove (3.20) under the additional assumption

$$
q<4,
$$

noting that for any $\alpha \geq \beta>0$ and $k \in\{1, \ldots, N-1\}$, the set of values $q$ satisfying (3.19) and (3.21) is non-empty. Moreover, by interpolating with the trivial estimate

$$
\left\|Q \check{F}_{\sigma}\right\|_{\infty} \leq(2 \pi)^{-N} \sqrt{\left|\mathbb{S}^{N-1}\right|}\|F\|_{L^{2}\left(\mathbb{S}^{N-1}\right)}
$$

we can remove the extra assumption (3.21) a posteriori.

In the following, the letter $C$ stands for positive and possibly different constants depending only on $N, k, \alpha, \beta$ and $a$. Let $F \in \mathcal{C}\left(\mathbb{S}^{N-1}\right)$ be a $G_{k}$-invariant function. Without loss of generality we assume that $\|F\|_{L^{2}\left(\mathbb{S}^{N-1}\right)}=1$. Using Lemma 3.9, we can write

$$
\begin{aligned}
& \left\|Q \check{F}_{\sigma}\right\|_{q}^{q}=\int_{\mathbb{R}^{N}}\left|\left[Q \check{F}_{\sigma}\right](x)\right|^{q} d x=\int_{\mathbb{R}^{N}} Q(x)\left|\check{F}_{\sigma}(x)\right|^{q} d x \\
& \leq C \int_{\mathbb{R}^{N}} Q(x) \int_{0}^{1} r^{(N-k-1) \frac{q}{2}}\left(1-r^{2}\right)^{\frac{k-2}{2} \frac{q}{2}}\left|\check{d \sigma}_{N-k}\left(r x^{(N-k)}\right)\right|^{q}\left|\check{d \sigma}{ }_{k}\left(\sqrt{1-r^{2}} x^{(k)}\right)\right|^{q} d r d x \\
& =C \int_{0}^{1} r^{(N-k-1) \frac{q}{2}}\left(1-r^{2}\right)^{\frac{k-2}{2} \frac{q}{2}} \int_{\mathbb{R}^{N}} Q(x)\left|\check{d \sigma}_{N-k}\left(r x^{(N-k)}\right)\right|^{q}\left|\check{d \sigma}_{k}\left(\sqrt{1-r^{2}} x^{(k)}\right)\right|^{q} d x d r \\
& =C \int_{0}^{1} r^{(N-k-1) \frac{q}{2}-(N-k)}\left(1-r^{2}\right)^{\frac{q}{4}(k-2)-\frac{k}{2}} H_{k}(r) d r,
\end{aligned}
$$

where we have used that $|Q|^{q}=Q$ due to the special choice of $Q$ as a characteristic function. In the last line we have set

$$
H_{k}(r):=\int_{\mathbb{R}^{N}} Q\left(\frac{x^{(N-k)}}{r}, \frac{x^{(k)}}{\sqrt{1-r^{2}}}\right)\left|\check{d \sigma_{N-k}}\left(x^{(N-k)}\right)\right|^{q}\left|\check{d \sigma}_{k}\left(x^{(k)}\right)\right|^{q} d x .
$$


Using the definition of $Q$ and the estimate (3.14), we deduce that

$$
\begin{aligned}
& H_{k}(r)=\int_{\mathbb{R}^{k}}\left|\check{d \sigma} \sigma_{k}\left(x^{(k)}\right)\right|^{q} \int_{\left|x^{(N-k)}\right| \leq \operatorname{armax}\left\{\left(\frac{\left|x^{(k)}\right|}{\sqrt{1-r^{2}}}\right)^{-\alpha},\left(\frac{\left|x^{(k)}\right|}{\sqrt{1-r^{2}}}\right)^{-\beta}\right\}}\left|\check{d \sigma}_{N-k}\left(x^{(N-k)}\right)\right|^{q} d x^{(N-k)} d x^{(k)} \\
& \leq C \int_{\mathbb{R}^{k}}\left(1+\left|x^{(k)}\right|\right)^{q \frac{1-k}{2}} \int_{\left\{\left(\frac{\left|x^{(k)}\right|}{\sqrt{1-r^{2}}}\right)^{-\alpha}\left(\frac{\left|x^{(k)}\right|}{\sqrt{1-r^{2}}}\right)^{-\beta}\right\}}\left(1+\left|x^{(N-k)}\right|\right)^{q \frac{1-(N-k)}{2}} d x^{(N-k)} d x^{(k)} \\
& \left|x^{(N-k)}\right| \leq \operatorname{armax}\left\{\left(\frac{\left|x^{(k)}\right|}{\sqrt{1-r^{2}}}\right)^{-\alpha},\left(\frac{\left|x^{(k)}\right|}{\sqrt{1-r^{2}}}\right)^{-\beta}\right\} \\
& =C \int_{0}^{\infty} s^{k-1}(1+s)^{-\frac{q}{2}(k-1)} \int_{0}^{\operatorname{armax}\left\{\left(\frac{\sqrt{1-r^{2}}}{s}\right)^{\alpha},\left(\frac{\sqrt{1-r^{2}}}{s}\right)^{\beta}\right\}} t^{N-k-1}(1+t)^{-\frac{q}{2}(N-k-1)} d t d s \\
& =C \int_{0}^{\sqrt{1-r^{2}}} s^{k-1}(1+s)^{-\frac{q}{2}(k-1)} \int_{0}^{\operatorname{ar}\left(1-r^{2}\right)^{\frac{\alpha}{2} s^{-\alpha}}} t^{N-k-1}(1+t)^{-\frac{q}{2}(N-k-1)} d t d s \\
& +C \int_{\sqrt{1-r^{2}}}^{\infty} s^{k-1}(1+s)^{-\frac{q}{2}(k-1)} \int_{0}^{\operatorname{ar}\left(1-r^{2}\right)^{\frac{\beta}{2}} s^{-\beta}} t^{N-k-1}(1+t)^{-\frac{q}{2}(N-k-1)} d t d s,
\end{aligned}
$$

where the last equality follows since $\alpha \geq \beta>0$ by assumption and therefore

$$
\max \left\{\left(\frac{s}{\sqrt{1-r^{2}}}\right)^{-\alpha},\left(\frac{s}{\sqrt{1-r^{2}}}\right)^{-\beta}\right\}= \begin{cases}\left(1-r^{2}\right)^{\frac{\alpha}{2}} s^{-\alpha} & 0<s<\sqrt{1-r^{2}} \\ \left(1-r^{2}\right)^{\frac{\beta}{2}} s^{-\beta}, & s \geq \sqrt{1-r^{2}}\end{cases}
$$

Combining (3.22) and (3.23), we can estimate

$$
\left\|Q \check{F}_{\sigma}\right\|_{q}^{q} \leq C\left(I_{k}^{(1)}+I_{k}^{(2)}\right)
$$

with

$$
I_{k}^{(i)}:=\int_{0}^{1} r^{(N-k-1) \frac{q}{2}-(N-k)}\left(1-r^{2}\right)^{\frac{q}{4}(k-2)-\frac{k}{2}} H_{k}^{(i)}(r) d r \quad \text { for } i=1,2
$$

and

$$
\begin{aligned}
& H_{k}^{(1)}(r):=\int_{\sqrt{1-r^{2}}}^{\infty} s^{k-1}(1+s)^{-\frac{q}{2}(k-1)} \int_{0}^{\operatorname{ar}\left(1-r^{2}\right)^{\frac{\beta}{2} s^{-\beta}}} t^{N-k-1}(1+t)^{-\frac{q}{2}(N-k-1)} d t d s, \\
& H_{k}^{(2)}(r):=\int_{0}^{\sqrt{1-r^{2}}} s^{k-1}(1+s)^{-\frac{q}{2}(k-1)} \int_{0}^{a r\left(1-r^{2}\right)^{\frac{\alpha}{2} s^{-\alpha}}} t^{N-k-1}(1+t)^{-\frac{q}{2}(N-k-1)} d t d s .
\end{aligned}
$$


We first estimate $I_{k}^{1}$, and we note that

$$
\begin{aligned}
& H_{k}^{(1)}(r) \leq \int_{\sqrt{1-r^{2}}}^{\infty} s^{\left(1-\frac{q}{2}\right)(k-1)} \int_{0}^{\operatorname{ar}\left(1-r^{2}\right)^{\frac{\beta}{2}} s^{-\beta}} t^{N-k-1} d t d s \\
& \quad \leq C r^{N-k}\left(1-r^{2}\right)^{\frac{\beta}{2}(N-k)} \int_{\sqrt{1-r^{2}}}^{\infty} s^{\left(1-\frac{q}{2}\right)(k-1)-\beta(N-k)} d s \leq C r^{N-k}\left(1-r^{2}\right)^{\left(1-\frac{q}{2}\right) \frac{k-1}{2}+\frac{1}{2}}
\end{aligned}
$$

for $0<r<1$. Here we used in the last step that $\left(1-\frac{q}{2}\right)(k-1)-\beta(N-k)<-1$ since by 3.19 we have

$$
\beta>\frac{1}{N-1} \quad \text { in case } k=1 \quad \text { and } \quad q>\frac{2 k-2 \beta(N-k)}{k-1} \quad \text { if } 2 \leq k \leq N-1 .
$$

Combining (3.25) and (3.26), we conclude that

$$
I_{k}^{(1)} \leq C \int_{0}^{1} r^{(N-k-1) \frac{q}{2}}\left(1-r^{2}\right)^{\frac{q}{4}(k-2)-\frac{k}{2}+\left(1-\frac{q}{2}\right) \frac{k-1}{2}+\frac{1}{2}} d r=C \int_{0}^{1} r^{(N-k-1) \frac{q}{2}}\left(1-r^{2}\right)^{-\frac{q}{4}} d r,
$$

where the integral on the RHS is finite due to 3.21 . As a consequence, $I_{k}^{(1)} \leq C_{1}$ with a constant $C_{1}=C_{1}(N, k, \beta, a)>0$.

Next we consider $I_{k}^{(2)}$, and we distinguish the following cases.

Case 1: $q(N-k-1)<2(N-k)$.

In this case we estimate as follows:

$$
\begin{aligned}
H_{k}^{(2)}(r) & \leq \int_{0}^{\sqrt{1-r^{2}}} s^{k-1} \int_{0}^{\operatorname{ar}\left(1-r^{2}\right)^{\frac{\alpha}{2}} s^{-\alpha}} t^{N-k-1-\frac{q}{2}(N-k-1)} d t d s \\
& \leq C r^{N-k-\frac{q}{2}(N-k-1)}\left(1-r^{2}\right)^{\frac{\alpha}{2}(N-k)-\frac{\alpha}{2} \frac{q}{2}(N-k-1)} \int_{0}^{\sqrt{1-r^{2}}} s^{k-1-\alpha(N-k)+\alpha \frac{q}{2}(N-k-1)} d s \\
& =C r^{N-k-\frac{q}{2}(N-k-1)}\left(1-r^{2}\right)^{\frac{k}{2}} \quad \text { for } r \in(0,1) .
\end{aligned}
$$

Here we used in the last step that $k-1-\alpha(N-k)+\alpha \frac{q}{2}(N-k-1)>-1$ since by (3.19) we have

$$
q>\frac{2(N-k)-\frac{2 k}{\alpha}}{N-k-1} \quad \text { in case } k<N-1 \quad \text { and } \quad \alpha<N-1 \quad \text { if } k=N-1 .
$$

We thus conclude that

$$
I_{k}^{(2)}=\int_{0}^{1} r^{(N-k-1) \frac{q}{2}-(N-k)}\left(1-r^{2}\right)^{\frac{q}{4}(k-2)-\frac{k}{2}} H_{k}^{(2)}(r) d r \leq C \int_{0}^{1}\left(1-r^{2}\right)^{\frac{q}{4}(k-2)} d r<\infty .
$$

We now consider 
Case 2: $q(N-k-1) \geq 2(N-k)$.

In this case we choose $\delta \in\left(\frac{q}{2}(N-k-1)-(N-k), \min \left\{\frac{q}{2}(N-k-1), \frac{q}{2}(N-k-1)+\frac{k}{\alpha}-(N-k)\right\}\right)$, and we estimate as follows:

$$
\begin{aligned}
& H_{k}^{(2)}(r) \leq \int_{0}^{\sqrt{1-r^{2}}} s^{k-1} \int_{0}^{\operatorname{ar}\left(1-r^{2}\right)^{\frac{\alpha}{2}} s^{-\alpha}} t^{N-k-1}(1+t)^{\delta-\frac{q}{2}(N-k-1)} d t d s \\
& \leq \int_{0}^{\sqrt{1-r^{2}}} s^{k-1} \int_{0}^{\operatorname{ar}\left(1-r^{2}\right)^{\frac{\alpha}{2}} s^{-\alpha}} t^{N-k-1+\delta-\frac{q}{2}(N-k-1)} d t d s \\
& \leq C r^{N-k+\delta-\frac{q}{2}(N-k-1)}\left(1-r^{2}\right)^{\frac{\alpha}{2}(N-k+\delta)-\frac{\alpha}{2} \frac{q}{2}(N-k-1)} \int_{0}^{\sqrt{1-r^{2}}} s^{k-1-\alpha(N-k+\delta)+\alpha \frac{q}{2}(N-k-1)} d s \\
& =C r^{N-k+\delta-\frac{q}{2}(N-k-1)}\left(1-r^{2}\right)^{\frac{k}{2}} \quad \text { for } r \in(0,1) .
\end{aligned}
$$

We thus conclude that

$$
I_{k}^{(2)}=\int_{0}^{1} r^{(N-k-1) \frac{q}{2}-(N-k)}\left(1-r^{2}\right)^{\frac{q}{4}(k-2)-\frac{k}{2}} H_{k}^{(2)}(r) d r \leq C \int_{0}^{1} r^{\delta}\left(1-r^{2}\right)^{\frac{q}{4}(k-2)} d r<\infty
$$

Combining 3.27) and 3.28, we conclude that $I_{k}^{(2)} \leq C_{2}$ with a constant $C_{2}=C_{2}(N, k, \alpha, a)>$ 0 .

Going back to 3.24 , we deduce that

$$
\left\|Q \check{F}_{\sigma}\right\|_{q}^{q} \leq C\left(C_{1}+C_{2}\right)
$$

where the constant on the RHS only depends on $N, k, \alpha, \beta$ and $a$. The proof is thus finished.

We note that Theorem 3.2 is a direct consequence of Theorem 3.10 , since the assumptions of Theorem 3.2 imply those of Theorem 3.10 in the case $\alpha=\beta$.

Moreover, we have the following duality property.

Lemma 3.11. Suppose that a closed subgroup $G \subset O(N), q \geq 1$, and $Q \in L_{G}^{\infty}\left(\mathbb{R}^{N}\right)$ are given with the property that $(G, q, Q)$ is an admissible extension pair in the sense of Definition 3.1 . Then there exists a constant $C>0$ with

$$
\left\|\left.\widehat{Q f}\right|_{\mathbb{S}^{N-1}}\right\|_{L^{2}\left(\mathbb{S}^{N-1}\right)} \leq C\|f\|_{q^{\prime}} \quad \text { for every } f \in \mathcal{S}_{G} \text {. }
$$

In particular, this holds if $G=G_{k}$ and $N, k, \alpha, \beta, q$ and $Q$ satisfy the assumptions of Theorem 3.10 . 
Proof. Let $f \in \mathcal{S}_{G}$ and $F:=\left.\widehat{Q f}\right|_{\mathbb{S}^{N-1}} \in L^{2}\left(\mathbb{S}^{N-1}\right)$. Then we have

$$
\begin{aligned}
& \|F\|_{L^{2}\left(\mathbb{S}^{N-1}\right)}^{2}=\int_{\mathbb{S}^{N-1}} \widehat{Q f} \bar{F} d \sigma=(2 \pi)^{-\frac{N}{2}} \int_{\mathbb{S}^{N-1}} \int_{\mathbb{R}^{N}} e^{-i x \theta} f(x) Q(x) d x \overline{F(\theta)} d \sigma(\theta) \\
& =(2 \pi)^{-\frac{N}{2}} \int_{\mathbb{R}^{N}} f(x) \overline{\overline{Q(x)} \int_{\mathbb{S}^{N-1}} e^{i x \theta} F(\theta) d \sigma(\theta)} d x \leq\|f\|_{q^{\prime}}\left\|Q \check{F}_{\sigma}\right\|_{q} \leq C\|f\|_{q^{\prime}}\|F\|_{L^{2}\left(\mathbb{S}^{N-1}\right)}
\end{aligned}
$$

and therefore $\|F\|_{L^{2}\left(\mathbb{S}^{N-1}\right)} \leq C\|f\|_{q^{\prime}}$, as claimed.

\section{Resolvent estimates for $G$-invariant functions}

For $N \geq 3$, the radial outgoing fundamental solution of the Helmholtz equation $-\Delta u-u=\delta_{0}$ in $\mathbb{R}^{N}$ is given by

$$
\Phi(x):=\frac{\mathrm{i}}{4}(2 \pi|x|)^{\frac{2-N}{2}} H_{\frac{N-2}{2}}^{(1)}(|x|), \quad \text { for } x \in \mathbb{R}^{N} \backslash\{0\},
$$

where $H_{\frac{N-2}{2}}^{(1)}$ denotes the Hankel function of the first kind of order $\frac{N-2}{2}$. For a function $f \in$ $\mathcal{S}\left(\mathbb{R}^{N}\right)$ the convolution $u:=\Phi * f \in \mathcal{C}^{\infty}\left(\mathbb{R}^{N}\right)$ is a solution of the inhomogeneous Helmholtz equation $-\Delta u-u=f$ which satisfies the Sommerfeld outgoing radiation condition $\partial_{r} u(x)-$ $\mathrm{i} u(x)=o\left(|x|^{\frac{1-N}{2}}\right.$ ), as $|x| \rightarrow \infty$. Moreover, it is known (see [36]) that, in the sense of tempered distributions, the Fourier transform of $\Phi$ is given by

$$
\widehat{\Phi}(\xi)=(2 \pi)^{-\frac{N}{2}} \frac{1}{|\xi|^{2}-(1+\mathrm{i} 0)}:=(2 \pi)^{-\frac{N}{2}} \lim _{\varepsilon \rightarrow 0^{+}} \frac{1}{|\xi|^{2}-(1+\mathrm{i} \varepsilon)} .
$$

As a consequence of a classical estimate of Kenig, Ruiz and Sogge (see [43, Theorem 2.3]), the mapping $f \mapsto \Phi * f$ for $f \in \mathcal{S}\left(\mathbb{R}^{N}\right)$ extends as a continuous linear operator

$$
\mathcal{R}: L^{p^{\prime}}\left(\mathbb{R}^{N}\right) \rightarrow L^{p}\left(\mathbb{R}^{N}\right)
$$

for $\frac{2(N+1)}{N-1} \leq p \leq \frac{2 N}{N-2}$. Moreover, non-selfdual $\left(L^{r}, L^{p^{\prime}}\right)$-estimates for $\mathcal{R}$ were established by Gutiérrez in [37, Theorem 6]. The aim of this section is to establish a similar estimate for the operator $\mathcal{R}_{Q}$ defined by $\mathcal{R}_{Q}(f):=f \mapsto Q[\Phi *(Q f)]$, where $G$ is a closed subgroup of $O(N)$ and $Q \in L_{G}^{\infty}\left(\mathbb{R}^{N}\right)$ is a weight function. The main result of this section is the following generalization of Theorem 3.3 .

Theorem 3.12. Let $N \geq 3$, let $G \subset O(N)$ be a closed subgroup, let $Q \in L_{G}^{\infty}\left(\mathbb{R}^{N}\right)$, and let $q \in\left[1, \frac{2(N+1)}{N-1}\right]$ be such that $(G, q, Q)$ is an admissible extension triple. Moreover, let $p, r \in(1, \infty)$ satisfy

$$
\frac{N-2}{N} \leq \frac{1}{p}+\frac{1}{r}<\frac{q+2}{2 q} \frac{N-1}{N} .
$$

Suppose moreover that

$$
\max \left\{\frac{1}{r}, \frac{1}{p}\right\}<\frac{N-1}{2 N} \quad \text { if } q \geq 2
$$


and that

$\frac{2 q}{(N-1)(2-q)} \frac{1}{p}-\frac{(N-1) q-2(N-3)}{2 N(2-q)}<\frac{1}{r}<\frac{(N-1)(2-q)}{2 q} \frac{1}{p}+\frac{(N-1)[(N-1) q-2(N-3)]}{4 q N}$

if $q<2$. Then there exists $C>0$ such that

$$
\left\|\mathcal{R}_{Q} f\right\|_{r} \leq C\|f\|_{p^{\prime}} \quad \text { for all functions } f \in \mathcal{S}_{G}\left(\mathbb{R}^{N}\right) .
$$

REMARK 3.13. (i) In the special case $r=p$, the assumptions of Theorem 3.12 reduce to $p \in\left(\frac{2 N}{N-1} \frac{2 q}{q+2}, \frac{2 N}{N-2}\right]$. Hence Theorem 3.3 follows directly from Theorem 3.12 .

(ii) The assumption $q \in\left[1, \frac{2(N+1)}{N-1}\right]$ implies that condition 3.31) covers the (nonempty) condition $\frac{N-2}{N} \leq \frac{1}{p}+\frac{1}{r}<\frac{N-1}{N+1}$ considered in [37, Theorem 6].

(iii) Geometrically, the conditions (3.31), (3.32) and (3.33) can be formulated for the point $\left(\frac{1}{p}, \frac{1}{r}\right)$ to be contained in the trapezoid in $(0,1) \times(0,1)$ spanned by the points

$$
\left(\frac{N-3}{2 N}, \frac{N-1}{2 N}\right), \quad\left(\frac{N-1}{q N}, \frac{N-1}{2 N}\right), \quad\left(\frac{N-1}{2 N}, \frac{N-3}{2 N}\right) \quad \text { and } \quad\left(\frac{N-1}{2 N}, \frac{N-1}{q N}\right),
$$

with part of the boundary being excluded due to the fact that some of these inequalities are strict. In the case $q=2$, this trapezoid degenerates to a triangle.
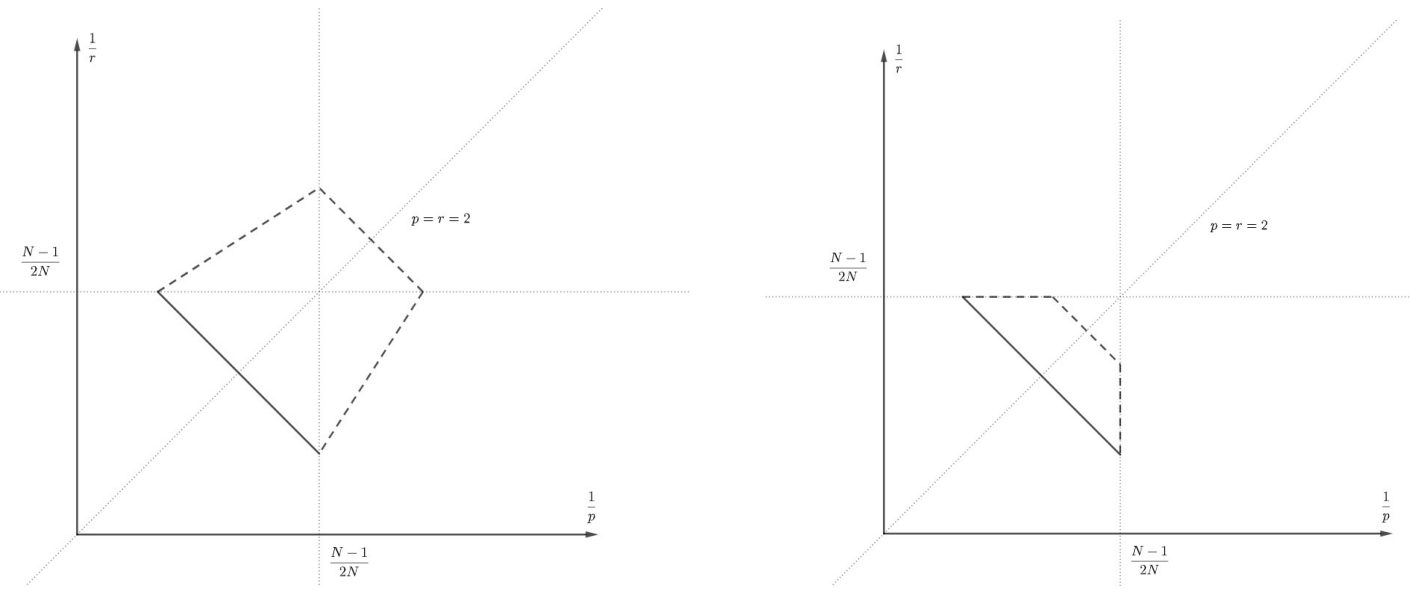

FiguRE 2. Reflected Riesz diagrams for $1 \leq q<2$ (left) and $q>2$ (right)

In order to prove Theorem 3.12, we adapt the strategy of [37] and [43, see also [31. Throughout the remainder of this section, we fix a closed subgroup $G \subset O(N)$ and $Q \in L_{G}^{\infty}\left(\mathbb{R}^{N}\right)$. We first note the following lemma which is a basic consequence of complex interpolation. 
Lemma 3.14. Let $1 \leq q<\infty$ and let $\rho \in \mathcal{S}$ be a radial function. Suppose furthermore that

$$
\begin{aligned}
\|Q[\rho *(Q u)]\|_{2} \leq C D\|u\|_{q^{\prime}} & & \text { and } \\
\|Q[\rho *(Q u)]\|_{\infty} \leq C D^{1-N}\|u\|_{1} & & \text { for all } u \in \mathcal{S}_{G}\left(\mathbb{R}^{N}\right)
\end{aligned}
$$

with constants $C, D>0$. Suppose furthermore that $p, r \in(1, \infty)$ satisfy

$$
\frac{d_{q, 1}}{p} \leq \frac{1}{r} \leq \min \left\{\frac{1}{2}+\frac{1}{q}-\frac{1}{p}, \frac{d_{q, 2}}{p}\right\}
$$

where

$$
d_{q, 1}=\min \left\{\frac{q}{2}, \frac{2}{q}\right\} \quad \text { and } \quad d_{q, 2}=\max \left\{\frac{q}{2}, \frac{2}{q}\right\}
$$

Then we have

$$
\|Q[\rho *(Q u)]\|_{r} \leq C D^{A_{r, p, q}}\|u\|_{p^{\prime}} \quad \text { for all } u \in \mathcal{S}_{G}\left(\mathbb{R}^{N}\right)
$$

with

$$
A_{r, p, q}:=\frac{2 q N}{q+2}\left(\frac{1}{r}+\frac{1}{p}\right)-(N-1) .
$$

Proof. Since $\rho \in \mathcal{S}$ is radial, the convolution with $\rho$ maps $G$-invariant functions to $G$ invariant functions. Moreover, by assumption we have

$$
\int_{\mathbb{R}^{N}} v Q[\rho *(Q u)] d x=\int_{\mathbb{R}^{N}} u Q[\rho *(Q v)] d x \leq\|u\|_{2}\|Q[\rho *(Q v)]\|_{2} \leq C D\|u\|_{2}\|v\|_{q^{\prime}}
$$

for all $u, v \in \mathcal{S}_{G}$. By duality, we therefore have

$$
\|Q[\rho *(Q u)]\|_{q} \leq C D\|u\|_{2} \quad \text { for all } u \in \mathcal{S}_{G} .
$$

Note that, by 3.37 , the point $\left(\frac{1}{p}, \frac{1}{r}\right)$ is contained in the closed symmetric triangle in $\mathbb{R}^{2}$ spanned by the points $\left(\frac{1}{q}, \frac{1}{2}\right),\left(\frac{1}{2}, \frac{1}{q}\right)$ and $(0,0)$. Hence we can write

$$
\frac{1}{p}=\frac{\lambda}{q}+\frac{\mu}{2}, \quad \frac{1}{r}=\frac{\lambda}{2}+\frac{\mu}{q} \quad \text { with } \quad \lambda, \mu \geq 0, \quad \lambda+\mu \leq 1 .
$$

With $\gamma:=1-(\lambda+\mu)$, we thus have

$$
\frac{1}{p^{\prime}}=\frac{\lambda}{q^{\prime}}+\frac{\mu}{2}+\frac{\gamma}{1}, \quad \frac{1}{r}=\frac{\lambda}{2}+\frac{\mu}{q}+\frac{\gamma}{\infty},
$$

so complex interpolation of the inequalities (3.35), 3.36) and (3.41) gives

$$
\|Q[\rho *(Q u)]\|_{r} \leq C D^{\lambda+\mu-\gamma(N-1)}\|u\|_{p^{\prime}}=C D^{1-\gamma N}\|u\|_{p^{\prime}} \quad \text { for all } u \in \mathcal{S}_{G} .
$$

Solving 3.42 yields $\lambda+\mu=\frac{2 q}{q+2}\left(\frac{1}{r}+\frac{1}{p}\right)$ and therefore $\gamma=1-\frac{2 q}{q+2}\left(\frac{1}{r}+\frac{1}{p}\right)$. Hence 3.39 follows from $(3.43)$.

Next we decompose the fundamental solution $\Phi$ as in [37] and [31]. For this we fix $\psi \in \mathcal{S}\left(\mathbb{R}^{N}\right)$ such that $\widehat{\psi} \in \mathcal{C}_{c}^{\infty}$ is radial, $0 \leq \widehat{\psi} \leq 1$ and $\widehat{\psi}=1$ for ||$\xi|-1| \leq \frac{1}{6}, \widehat{\psi}(\xi)=0$ for ||$\xi|-1| \geq \frac{1}{4}$. 
We then write

$$
\Phi=\Phi_{1}+\Phi_{2} \quad \text { with } \quad \Phi_{1}=\psi * \Phi, \quad \Phi_{2}=\Phi-\Phi_{1} .
$$

Accordingly, we write

$$
\mathcal{R}_{Q}=\mathcal{R}_{Q}^{1}+\mathcal{R}_{Q}^{2} \quad \text { with } \quad \mathcal{R}_{Q}^{i}(f):=Q\left[\Phi_{i} *(Q f)\right], \quad i=1,2 .
$$

As shown in [31, Section 3], we have

$$
\left|\Phi_{1}(x)\right| \leq C(1+|x|)^{\frac{1-N}{2}} \quad \text { for } x \in \mathbb{R}^{N}
$$

and

$$
\left|\Phi_{2}(x)\right| \leq C \min \left\{|x|^{2-N},|x|^{-N}\right\} \quad \text { for } x \in \mathbb{R}^{N} \backslash\{0\} .
$$

In particular, by the Hardy Littlewood Sobolev inequality, we have the following.

Lemma 3.15. For every pair of numbers $p, r \in(1, \infty)$ satisfying

$$
\frac{1}{p}+\frac{1}{r} \geq \frac{N-2}{N},
$$

the convolution operator $f \mapsto \Phi_{2} * f$ defines a bounded linear map from $L^{p^{\prime}}\left(\mathbb{R}^{N}\right) \rightarrow L^{r}\left(\mathbb{R}^{N}\right)$. Consequently, the operator $\mathcal{R}_{Q}^{2}$ also defines a bounded linear map from $L^{p^{\prime}}\left(\mathbb{R}^{N}\right) \rightarrow L^{r}\left(\mathbb{R}^{N}\right)$ in this case.

Next we turn to the operator $\mathcal{R}_{Q}^{1}$.

Proposition 3.16. Let $q \in[1, \infty)$ be given such that $(G, q, Q)$ is an admissible extension triple. Moreover, let $p, r \in(1, \infty)$ satisfy

$$
\frac{d_{q, 1}}{p} \leq \frac{1}{r} \leq \frac{d_{q, 2}}{p} \quad \text { and } \quad \frac{1}{p}+\frac{1}{r}<\frac{q+2}{2 q} \frac{N-1}{N}
$$

with $d_{q, 1}, d_{q, 2}$ defined in (3.38). Then there exists $C>0$ such that

$$
\left\|\mathcal{R}_{Q}^{1} f\right\|_{r} \leq C\|f\|_{p^{\prime}} \quad \text { for all functions } f \in \mathcal{S}_{G}\left(\mathbb{R}^{N}\right) .
$$

Proof. Let $\varphi \in \mathcal{S}\left(\mathbb{R}^{N}\right)$ be such that $\widehat{\varphi} \in \mathcal{C}_{c}^{\infty}\left(\mathbb{R}^{N}\right)$ is radial, $0 \leq \widehat{\varphi} \leq 1$ with $\widehat{\varphi} \equiv 1$ for ||$\xi|-1| \leq \frac{1}{4}$ and $\widehat{\varphi} \equiv 0$ for ||$\xi|-1| \geq \frac{1}{2}$. By construction of $\Phi_{1}$, we then have $\widehat{\Phi_{1}}=\widehat{\Phi_{1}} \widehat{\varphi}$, which means that $\Phi_{1}=(2 \pi)^{-\frac{N}{2}} \Phi_{1} * \varphi$ and therefore

$$
\mathcal{R}_{Q}^{1} f=Q\left[\Phi_{1} *(Q f)\right]=(2 \pi)^{-\frac{N}{2}} Q\left[\Phi_{1} * \varphi * Q f\right] \quad \text { for } f \in \mathcal{S} .
$$

Choose $\eta \in \mathcal{C}_{c}^{\infty}\left(\mathbb{R}^{N}\right)$ radial with $0 \leq \eta \leq 1, \eta(x)=1$ for $|x| \leq 1, \eta(x)=0$ for $|x| \geq 2$. Moreover we define $\psi_{j} \in \mathcal{C}_{c}^{\infty}\left(\mathbb{R}^{N}\right)$ by $\psi_{0}=\eta$ and $\psi_{j}(x)=\eta\left(2^{-j} x\right)-\eta\left(2^{-(j-1)} x\right)$ for $j \in \mathbb{N}, x \in \mathbb{R}^{N}$. Then we have the dyadic composition

$$
\Phi_{1}=\sum_{j=0}^{\infty} \Phi_{1}^{j} \quad \text { with } \quad \Phi_{1}^{j}=\psi_{j} \Phi_{1}
$$


Using (3.44), we find that

$$
\left\|\Phi_{1}^{j}\right\|_{\infty} \leq C 2^{-\frac{j(N-1)}{2}}, \quad \text { for all } j,
$$

where the constant $C>0$ is independent of $j$. Using that $\widehat{\Phi_{1}^{j}}$ is radial, we get, with Plancherel's theorem and Lemma 3.11 .

$$
\begin{aligned}
\left\|\left(\Phi_{1}^{j} * \varphi\right) *(Q f)\right\|_{2}^{2} & =C \int_{\mathbb{R}^{N}}\left|\widehat{\Phi_{1}^{j}}(\xi) \widehat{\varphi} \widehat{Q f}(\xi)\right|^{2} d \xi \leq\left. C \int_{\frac{1}{4}}^{\frac{7}{4}} r^{N-1} \widehat{\Phi_{1}^{j}}(r)\right|^{2} \int_{\mathbb{S}^{N-1}}|\widehat{Q f}(r \omega)|^{2} d \sigma(\omega) d r \\
& \leq C\left\|\Phi_{1}^{j}\right\|_{2}^{2}\|Q f\|_{q^{\prime}}^{2} \leq C 2^{j}\|Q f\|_{q^{\prime}}^{2} \quad \text { for all } f \in \mathcal{S}_{G},
\end{aligned}
$$

where the constant does not depend on $j$. Consequently, we thus have

$$
\left\|Q\left(\Phi_{1}^{j} * \varphi\right) *(Q f)\right\|_{2} \leq C 2^{\frac{j}{2}}\|Q f\|_{q^{\prime}} \quad \text { for all } f \in \mathcal{S}_{G}
$$

Moreover, we have

$$
\left\|\Phi_{1}^{j} * \varphi\right\|_{\infty} \leq\left\|\Phi_{1}^{j}\right\|_{\infty}\|\varphi\|_{1} \leq C 2^{-\frac{j(N-1)}{2}}, \quad \text { for all } j
$$

which implies that

$$
\left\|Q\left(\Phi_{1}^{j} * \varphi\right) *(Q f)\right\|_{\infty} \leq C 2^{-\frac{j(N-1)}{2}}\|Q f\|_{1} \leq C 2^{-\frac{j(N-1)}{2}}\|f\|_{1} \quad \text { for all } f \in \mathcal{S}_{G}
$$

Since the assumption 3.46 implies 3.37), we may apply Lemma 3.14 to the radial kernel $\Phi_{1}^{j} * \varphi \in \mathcal{S}\left(\mathbb{R}^{N}\right)$ and deduce that

$$
\left\|Q\left(\Phi_{1}^{j} * \varphi\right) *(Q f)\right\|_{r} \leq C 2^{\frac{j}{2} A_{r, p, q}}\|f\|_{p^{\prime}} \quad \text { for all } f \in \mathcal{S}_{G}
$$

with $A_{r, p, q}$ given in (3.40). By assumption (3.46), we have $A_{r, p, q}<0$. Since, as remarked above, $\Phi_{1}=(2 \pi)^{-\frac{N}{2}} \Phi_{1} * \varphi$, we deduce that

$$
\begin{aligned}
\left\|\mathcal{R}_{Q}^{1} f\right\|_{r} & =\left\|Q \Phi_{1} *(Q f)\right\|_{r}=(2 \pi)^{-\frac{N}{2}}\left\|Q\left(\Phi_{1} * \varphi\right) *(Q f)\right\|_{r} \\
& \leq(2 \pi)^{-\frac{N}{2}} \sum_{j=0}^{\infty}\left\|Q\left(\Phi_{1}^{j} * \varphi\right) *(Q f)\right\|_{r} \leq C_{0}\|f\|_{p^{\prime}} \quad \text { for all } f \in \mathcal{S}_{G}
\end{aligned}
$$

with $C_{0}=C(2 \pi)^{-\frac{N}{2}} \sum_{j=0}^{\infty} 2^{\frac{j}{2}} A_{r, p, q}<\infty$. The proof is finished.

We may now complete the

Proof of Theorem 3.12, Let $M$ denote the set of points $\left(\frac{1}{p}, \frac{1}{r}\right) \in(0,1) \times(0,1)$ such that (3.34 holds with some constant $C>0$. By combining Lemma 3.15 and Proposition 3.16 . we see that $\left(\frac{1}{p}, \frac{1}{r}\right) \in M$ if

$$
\frac{d_{q, 1}}{p} \leq \frac{1}{r} \leq \frac{d_{q, 2}}{p} \quad \text { and } \quad \frac{N-2}{N} \leq \frac{1}{p}+\frac{1}{r}<\frac{q+2}{2 q} \frac{N-1}{N} .
$$


Hence the closure of $M$ contains the points $\left(\frac{N-1}{q N}, \frac{N-1}{2 N}\right),\left(\frac{N-1}{2 N}, \frac{N-1}{q N}\right)$ and therefore also the line segment between these points. Moreover, by [43, Lemma 2.2(b)], $M$ also contains the open line segment between the points $\left(\frac{N-3}{2 N}, \frac{N-1}{2 N}\right)$ and $\left(\frac{N-1}{2 N}, \frac{N-3}{2 N}\right)$. Hence, if $q \geq 2$, complex interpolation yields that $M$ contains all points $\left(\frac{1}{p}, \frac{1}{r}\right) \in(0,1) \times(0,1)$ with the property that $\frac{1}{r}, \frac{1}{p}<\frac{N-1}{2 N}$ and that

$$
\frac{N-2}{N} \leq \frac{1}{p}+\frac{1}{r}<\frac{q+2}{2 q} \frac{N-1}{N}
$$

i.e., all points $\left(\frac{1}{p}, \frac{1}{r}\right) \in(0,1) \times(0,1)$ satisfying 3.31 and 3.32 . Hence the theorem is proved in the case $q \geq 2$.

If $q<2$, complex interpolation yields that $M$ contains all points $\left(\frac{1}{p}, \frac{1}{r}\right) \in(0,1) \times(0,1)$ satisfying 3.49 and with the property that $\left(\frac{1}{p}, \frac{1}{q}\right)$ lies above the line through the points $\left(\frac{N-1}{2 N}, \frac{N-3}{2 N}\right)$, $\left(\frac{N-1}{q N}, \frac{N-1}{2 N}\right)$ and below the line through the points $\left(\frac{N-3}{2 N}, \frac{N-1}{2 N}\right),\left(\frac{N-1}{2 N}, \frac{N-1}{q N}\right)$. This is precisely the set of points $\left(\frac{1}{p}, \frac{1}{q}\right) \in(0,1) \times(0,1)$ satisfying 3.31 and 3.33 . The proof is thus also finished in this case.

\section{Nonvanishing for $G$-invariant functions}

Our next aim is to deduce a nonvanishing theorem for the operator $\mathcal{R}_{Q}$ and $G$-invariant functions, where again $G \subset O(N)$ is a closed subgroup and $Q \in L_{G}^{\infty}\left(\mathbb{R}^{N}\right)$ is a given weight function. We restate Theorem 3.4 for the reader's convenience.

Theorem 3.17. Let $N \geq 3$, let $G \subset O(N)$ be a closed subgroup, let $Q \in L_{G}^{\infty}\left(\mathbb{R}^{N}\right)$, and let $q \in\left[1, \frac{2(N+1)}{N-1}\right]$ be such that $(G, q, Q)$ is an admissible extension triple. Moreover, let $p \in\left(\frac{2 N}{N-1} \frac{2 q}{q+2}, \frac{2 N}{N-2}\right]$. Then for every bounded sequence $\left(v_{n}\right)_{n} \subset L_{G}^{p^{\prime}}\left(\mathbb{R}^{N}\right)$ satisfying

$$
\left|\limsup _{n \rightarrow \infty} \int_{\mathbb{R}^{N}} v_{n} \mathcal{R}_{Q}\left(v_{n}\right) d x\right|>0,
$$

there exist - after passing to a subsequence - numbers $R, \zeta>0$ and a sequence of points $\left(x_{n}\right)_{n \in \mathbb{N}} \subset \mathbb{R}^{N}$ with

$$
\int_{B_{R}\left(x_{n}\right)}\left|Q v_{n}(x)\right|^{p^{\prime}} d x \geq \zeta, \quad \text { for all } n .
$$

The remainder of this section is devoted to the proof of this theorem. For this we fix $N \geq 3$ and $q \in\left[1, \frac{2(N+1)}{N-1}\right]$ such that $(G, q, Q)$ is an admissible extension triple. Moreover, we keep using the notation of the previous section, so we write $\Phi=\Phi_{1}+\Phi_{2}$ and $\mathcal{R}_{Q}=\mathcal{R}_{Q}^{1}+\mathcal{R}_{Q}^{2}$. We need to analyse the operators $\mathcal{R}_{Q}^{1}$ and $\mathcal{R}_{Q}^{2}$ separately. We start by proving the following variant of Proposition 3.16 for the operator $\mathcal{R}_{Q}^{1}$.

Proposition 3.18. Let $\varphi \in \mathcal{S}\left(\mathbb{R}^{N}\right)$ be such that $\widehat{\varphi} \in \mathcal{C}_{c}^{\infty}\left(\mathbb{R}^{N}\right)$ is radial, $0 \leq \widehat{\varphi} \leq 1$ with $\widehat{\varphi} \equiv 1$ for ||$\xi|-1| \leq \frac{1}{4}$ and $\widehat{\varphi} \equiv 0$ for ||$\xi|-1| \geq \frac{1}{2}$. 
Moreover, let $\eta \in \mathcal{C}_{c}^{\infty}\left(\mathbb{R}^{N}\right)$ be radial with $0 \leq \eta \leq 1, \eta(x)=1$ for $|x| \leq 1, \eta(x)=0$ for $|x| \geq 2$, and let $\eta_{k}(x)=\eta\left(2^{-k} x\right)$ for $k \in \mathbb{N}$.

Finally, let $p \in\left(\frac{2 N}{N-1} \frac{2 q}{q+2}, \infty\right)$ and $A_{p, q}:=\frac{N}{p} \frac{4 q}{q+2}+1-N<0$. Then there exists $C>0$ such that for $k \geq 1$ we have

$$
\left\|Q\left(\left[\left(1-\eta_{k}\right) \Phi_{1}\right] * \varphi *(Q f)\right)\right\|_{p} \leq C \frac{2^{\frac{k+1}{2} A_{p, q}}}{1-2^{\frac{A_{p, q}}{2}}}\|f\|_{p^{\prime}}
$$

for all functions $f \in \mathcal{S}_{G}$.

Proof. Let $k \in \mathbb{N}$. Using the given function $\eta$, we let $\Phi_{1}^{j}, j \in \mathbb{N} \cup\{0\}$ be defined as in the proof of Proposition 3.16. The proof of this proposition yields, in particular, inequality (3.47) with $r=p$, which writes as

$$
\left\|Q\left[\left(\Phi_{1}^{j} * \varphi\right) *(Q f)\right]\right\|_{p} \leq C 2^{\frac{j}{2} A_{p, q}}\|f\|_{p^{\prime}} \quad \text { for } j \in \mathbb{N} \text { and all functions } f \in \mathcal{S}_{G}
$$

with $A_{p, q}:=A p, p, q=\frac{N}{p} \frac{4 q}{q+2}+1-N$, cf. 3.40 . Moreover, by construction, we have the dyadic decomposition

$$
\left(1-\eta_{k}\right) \Phi_{1}=\sum_{j=k+1}^{\infty} \Phi_{1}^{j}
$$

and therefore

$$
\begin{aligned}
\left\|Q\left(\left[\left(1-\eta_{k}\right) \Phi_{1}\right] * \varphi *(Q f)\right)\right\|_{p} \leq \sum_{j=k+1}^{\infty}\left\|Q\left[\left(\Phi_{1}^{j} * \varphi\right) *(Q f)\right]\right\|_{p} & \leq C\|f\|_{p^{\prime}} \sum_{j=k+1}^{\infty} 2^{\frac{j}{2} A_{p, q}} \\
& =C \frac{2^{\frac{k+1}{2} A_{p, q}}}{1-2^{\frac{A_{p, q}}{2}}}\|f\|_{p^{\prime}},
\end{aligned}
$$

for all functions $f \in \mathcal{S}_{G}$, as claimed.

LEMmA 3.19. Let $p>\frac{2 N}{N-1} \frac{2 q}{q+2}$ and suppose that $\left(v_{n}\right)_{n} \subset \mathcal{S}_{G}$ is an $L^{p^{\prime}}$-bounded sequence with

$$
\lim _{n \rightarrow \infty} \sup _{y \in \mathbb{R}^{N}} \int_{B_{\rho}(y)}\left|Q v_{n}\right|^{p^{\prime}} d x=0, \quad \text { for all } \rho>0 .
$$

Then

$$
\int_{\mathbb{R}^{N}} Q v_{n}\left[\Phi_{1} *\left(Q v_{n}\right)\right] d x \rightarrow 0, \quad \text { as } n \rightarrow \infty .
$$

ProOF. Let, as in the assumptions of Proposition 3.18, $\varphi \in \mathcal{S}\left(\mathbb{R}^{N}\right)$ be such that $\widehat{\varphi} \in$ $\mathcal{C}_{c}^{\infty}\left(\mathbb{R}^{N}\right)$ is radial, $0 \leq \widehat{\varphi} \leq 1$ with $\widehat{\varphi} \equiv 1$ for ||$\xi|-1| \leq \frac{1}{4}$ and $\widehat{\varphi} \equiv 0$ for ||$\xi|-1| \geq \frac{1}{2}$. Moreover, let $w_{n}=\varphi *\left(Q v_{n}\right)$. Then we have

$$
\left\|w_{n}\right\|_{p^{\prime}} \leq\|\varphi\|_{1}\left\|Q v_{n}\right\|_{p^{\prime}} \leq\|\varphi\|_{1}\|Q\|_{\infty}\left\|v_{n}\right\|_{p^{\prime}}
$$

for all $n \in \mathbb{N}$ by Young's inequality, so $\left(w_{n}\right)_{n}$ is also a bounded sequence in $L^{p^{\prime}}\left(\mathbb{R}^{N}\right)$ by assumption. Since $\widehat{\Phi_{1}}=\widehat{\Phi_{1}} \widehat{\varphi}$, we have $(2 \pi)^{\frac{N}{2}} \Phi_{1}=\Phi_{1} * \varphi$. Therefore, with $\eta_{k}$ defined as in 
Proposition 3.18, we can write

$$
\begin{aligned}
(2 \pi)^{\frac{N}{2}} \int_{\mathbb{R}^{N}} Q v_{n}\left[\Phi_{1} *\left(Q v_{n}\right)\right] d x & =\int_{\mathbb{R}^{N}} Q v_{n}\left[\Phi_{1} * \varphi *\left(Q v_{n}\right)\right] d x \\
& =\int_{\mathbb{R}^{N}} Q v_{n}\left[\eta_{k} \Phi_{1} * \varphi *\left(Q v_{n}\right)\right] d x+\int_{\mathbb{R}^{N}} Q v_{n}\left[\left(1-\eta_{k}\right) \Phi_{1} * \varphi *\left(Q v_{n}\right)\right] d x,
\end{aligned}
$$

for every $n, k \in \mathbb{N}$, where

$$
\left|\int_{\mathbb{R}^{N}} Q v_{n}\left[\left(1-\eta_{k}\right) \Phi_{1} * \varphi *\left(Q v_{n}\right)\right] d x\right| \leq\left\|v_{n}\right\|_{p^{\prime}}\left\|Q\left(\left[\left(1-\eta_{k}\right) \Phi_{1}\right] * \varphi *\left(Q v_{n}\right)\right)\right\|_{p} \leq C \frac{2^{\frac{k+1}{2} A_{p, q}}}{1-2^{\frac{A_{p, q}}{2}}}\left\|v_{n}\right\|_{p^{\prime}}^{2}
$$

by Hölder's inequality and Proposition 3.18 . Since $A_{p, q}<0$, it follows that

$$
\sup _{n \in \mathbb{N}}\left|\int_{\mathbb{R}^{N}} Q v_{n}\left[\left(1-\eta_{k}\right) \Phi_{1} * \varphi *\left(Q v_{n}\right)\right] d x\right| \rightarrow 0, \quad \text { as } k \rightarrow \infty .
$$

For fixed $k \in \mathbb{N}$, we now choose $R=2^{k+1}$, which implies that $\eta_{k} \equiv 0$ on $\mathbb{R}^{N} \backslash B_{R}$. Decomposing $\mathbb{R}^{N}$ into disjoint $N$-cubes $\left\{Z_{l}\right\}_{l \in \mathbb{N}}$ of side length $R$, and considering for each $l$ the $N$ - cube $Z_{l}^{\prime}$ with the same center as $Z_{l}$ but with side length $3 R$, we find

$$
\begin{aligned}
& \left|\int_{\mathbb{R}^{N}} Q v_{n}\left[\left(\eta_{k} \Phi_{1}\right) * \varphi *\left(Q v_{n}\right)\right] d x\right|=\left|\int_{\mathbb{R}^{N}} Q v_{n}\left[\left(\eta_{k} \Phi_{1}\right) * w_{n}\right] d x\right| \\
& \leq \sum_{l=1}^{\infty} \int_{Z_{l}}\left(\int_{|x-y|<R}\left|\Phi_{1}(x-y)\right|\left|Q v_{n}(x)\right|\left|w_{n}(y)\right| d x\right) d y \\
& \leq\left\|\Phi_{1}\right\|_{L^{\infty}} \sum_{l=1}^{\infty} \int_{Z_{l}^{\prime}}\left|Q v_{n}\right| d x \int_{Z_{l}^{\prime}}\left|w_{n}\right| d x \\
& \leq\left\|\Phi_{1}\right\|_{L^{\infty}}\left[\sum_{l=1}^{\infty}\left(\int_{Z_{l}^{\prime}}\left|w_{n}\right| d x\right)^{p^{\prime}}\right]^{\frac{1}{p^{\prime}}}\left[\sum_{l=1}^{\infty}\left(\int_{Z_{l}^{\prime}}\left|Q v_{n}\right| d x\right)^{p}\right]^{\frac{1}{p}} \\
& \leq\left\|\Phi_{1}\right\|_{L^{\infty}}(3 R)^{\frac{2 N}{p}}\left[\sum_{l=1}^{\infty} \int_{Z_{l}^{\prime}}\left|w_{n}\right|^{p^{\prime}} d x\right]^{\frac{1}{p^{\prime}}}\left[\sum_{l=1}^{\infty}\left(\int_{Z_{l}^{\prime}}\left|Q v_{n}\right|^{p^{\prime}} d x\right)^{\frac{p}{p^{\prime}}}\right]^{\frac{1}{p}} \\
& \leq\left\|\Phi_{1}\right\|_{L^{\infty}}(3 R)^{\frac{2 N}{p}} 3^{\frac{N}{p^{\prime}}}\left\|w_{n}\right\|_{L^{p^{\prime}}}\left[\sup _{l \in \mathbb{N}} \int_{Z_{l}^{\prime}}\left|Q v_{n}\right|^{p^{\prime}} d x\right]^{\frac{p}{p^{\prime}}-1}\left[\sum_{l=1}^{\infty} \int_{Z_{l}^{\prime}}\left|Q v_{n}\right|^{p^{\prime}} d x\right]^{\frac{1}{p}} \\
& \leq\left\|\Phi_{1}\right\|_{L^{\infty}}(3 R)^{\frac{2 N}{p}} 3^{N}\left\|w_{n}\right\|_{L^{p^{\prime}}}\left[\sup _{y \in \mathbb{R}^{N}} \int_{B_{3 R \sqrt{N}}(y)}\left|Q v_{n}\right|^{p^{\prime}} d x\right]^{\frac{p}{p^{\prime}}-1}\left\|Q v_{n}\right\|_{p^{\prime}}^{\frac{p^{\prime}}{p}} \\
& \leq\left\|\Phi_{1}\right\|_{L^{\infty}}\|Q\|_{\infty}^{1+\frac{p^{\prime}}{p}}\|\varphi\|_{1}(3 R)^{\frac{2 N}{p}} 3^{N}\left\|v_{n}\right\|_{p^{\prime}}^{1+\frac{p^{\prime}}{p}}\left[\sup _{y \in \mathbb{R}^{N}} B_{3 R \sqrt{N}(y)}\left|Q v_{n}\right|^{p^{\prime}} d x\right]^{\frac{p}{p^{\prime}}-1},
\end{aligned}
$$


where we used (3.51) in the last step. By assumption, it now follows that

$$
\int_{\mathbb{R}^{N}} Q v_{n}\left[\left(\eta_{k} \Phi_{1}\right) * \varphi *\left(Q v_{n}\right)\right] d x \rightarrow 0 \quad \text { as } n \rightarrow \infty \text { for every } k \in \mathbb{N} .
$$

The claim now follows by combining (3.52) and (3.53).

Regarding $\Phi_{2}$ we make use of the following variant of [Y1, Theorem 2.5].

Lemma 3.20. Let $2<p \leq \frac{2 N}{N-2}$ and suppose that $\left(v_{n}\right)_{n}$ is a bounded sequence in $L^{p^{\prime}}\left(\mathbb{R}^{N}\right)$ such that

$$
\lim _{n \rightarrow \infty} \sup _{y \in \mathbb{R}^{N}} \int_{B_{\rho}(y)}\left|v_{n}\right|^{p^{\prime}} d x=0, \quad \text { for all } \rho>0 .
$$

Then

$$
\int_{\mathbb{R}^{N}} v_{n}\left[\Phi_{2} * v_{n}\right] d x \rightarrow 0 \quad \text { as } n \rightarrow \infty
$$

Proof. The claim follows from [Y1, Theorem 2.5] in the case where $v_{n} \in \mathcal{S}$ for every $n \in \mathbb{N}$. If $\left(v_{n}\right)_{n}$ is an arbitrary bounded sequence in $L^{p^{\prime}}\left(\mathbb{R}^{N}\right)$, we first recall that, by Lemma 3.15 , there exists a constant $C>0$ with

$$
\left\|\Phi_{2} * v\right\|_{p} \leq C\|v\|_{p^{\prime}} \quad \text { for every } v \in L^{p^{\prime}}\left(\mathbb{R}^{N}\right) .
$$

Moreover we choose, by density, $\tilde{v}_{n} \in \mathcal{S}$ with $\left\|v_{n}-\tilde{v}_{n}\right\|_{p^{\prime}} \leq \frac{1}{n}$ for every $n \in \mathbb{N}$. The assumption then implies that also

$$
\lim _{n \rightarrow \infty} \sup _{y \in \mathbb{R}^{N}} \int_{B_{\rho}(y)}\left|\tilde{v}_{n}\right|^{p^{\prime}} d x=0, \quad \text { for all } \rho>0
$$

and therefore

$$
\int_{\mathbb{R}^{N}} \tilde{v}_{n}\left[\Phi_{2} * \tilde{v}_{n}\right] d x \rightarrow 0, \quad \text { as } n \rightarrow \infty
$$

by [Y1, Theorem 2.5]. Moreover,

$$
\begin{aligned}
& \left|\int_{\mathbb{R}^{N}}\left[v_{n}\left(\Phi_{2} * v_{n}\right)-\tilde{v}_{n}\left(\Phi_{2} * \tilde{v}_{n}\right)\right] d x\right|=\left|\int_{\mathbb{R}^{N}}\left(v_{n}-\tilde{v}_{n}\right) \Phi_{2} *\left(v_{n}+\tilde{v}_{n}\right) d x\right| \\
& \leq C\left\|v_{n}-\tilde{v}_{n}\right\|_{p^{\prime}}\left\|v_{n}+\tilde{v}_{n}\right\|_{p^{\prime}} \leq \frac{C\left(1+\frac{1}{n}\right)\left\|v_{n}\right\|_{p^{\prime}}}{n} \rightarrow 0 \quad \text { as } n \rightarrow \infty
\end{aligned}
$$

and thus also

$$
\int_{\mathbb{R}^{N}} v_{n}\left[\Phi_{2} * v_{n}\right] d x \rightarrow 0 \quad \text { as } n \rightarrow \infty
$$

as claimed.

We are now in position to finish the proof of Theorem 3.17 
Proof of Theorem 3.17. Let $\left(v_{n}\right)_{n} \subset L_{G}^{p^{\prime}}\left(\mathbb{R}^{N}\right)$ be a bounded sequence, and suppose by contradiction that 3.50 does not hold. Then we have

$$
\lim _{n \rightarrow \infty} \sup _{y \in \mathbb{R}^{N}} \int_{B_{\rho}(y)}\left|Q v_{n}\right|^{p^{\prime}} d x=0, \quad \text { for all } \rho>0 .
$$

By density, we may choose $\tilde{v}_{n} \in \mathcal{S}_{G}$ with $\left\|v_{n}-\tilde{v}_{n}\right\|_{p^{\prime}} \leq \frac{1}{n}$ for every $n \in \mathbb{N}$, which implies that $\left\|Q v_{n}-Q \tilde{v}_{n}\right\|_{p^{\prime}} \leq \frac{\|Q\|_{\infty}}{n}$ for all $n$ and therefore also

$$
\lim _{n \rightarrow \infty} \sup _{y \in \mathbb{R}^{N}} \int_{B_{\rho}(y)}\left|Q \tilde{v}_{n}\right|^{p^{\prime}} d x=0, \quad \text { for all } \rho>0 .
$$

Combining Lemma 3.19 (applied to $\tilde{v}_{n}$ ) and Lemma 3.20 (applied to $Q \tilde{v}_{n}$ ), we then deduce that

$$
\int_{\mathbb{R}^{N}} \tilde{v}_{n} \mathcal{R}_{Q} \tilde{v}_{n} d x=\int_{\mathbb{R}^{N}} Q \tilde{v}_{n}\left[\Phi_{1} *\left(Q \tilde{v}_{n}\right)\right] d x+\int_{\mathbb{R}^{N}} Q \tilde{v}_{n}\left[\Phi_{2} *\left(Q \tilde{v}_{n}\right)\right] d x \rightarrow 0 \quad \text { as } n \rightarrow \infty .
$$

Moreover, by Theorem 3.3 we have

$$
\begin{aligned}
& \left|\int_{\mathbb{R}^{N}}\left[v_{n} \mathcal{R}_{Q} v_{n}-\tilde{v}_{n} \mathcal{R}_{Q} \tilde{v}_{n}\right] d x\right|=\left|\int_{\mathbb{R}^{N}}\left(v_{n}-\tilde{v}_{n}\right) \mathcal{R}_{Q}\left(v_{n}+\tilde{v}_{n}\right) d x\right| \\
& \leq\left\|v_{n}-\tilde{v}_{n}\right\|_{p^{\prime}}\left\|\mathcal{R}_{Q}\left(v_{n}+\tilde{v}_{n}\right)\right\|_{p} \leq C\left\|v_{n}-\tilde{v}_{n}\right\|_{p^{\prime}}\left\|v_{n}+\tilde{v}_{n}\right\|_{p} \\
& \rightarrow 0 \quad \text { as } n \rightarrow \infty
\end{aligned}
$$

Consequently, we also have that $\int_{\mathbb{R}^{N}} v_{n} \mathcal{R}_{Q} v_{n} d x \rightarrow 0$ as $n \rightarrow \infty$, contrary to the assumption. The claim thus follows.

\section{Dual variational framework and $G$-invariant solutions}

Let $G \subset O(N)$ be a fixed closed subgroup, and let $Q \in L_{G}^{\infty}\left(\mathbb{R}^{N}\right)$ be a nonnegative fixed weight function with $Q \not \equiv 0$. We now focus our attention to the equation

$$
-\Delta u-u=Q(x)|u|^{p-2} u, \quad u \in L^{p}\left(\mathbb{R}^{N}\right) .
$$

To prove the existence of nontrivial real-valued solutions of (3.54), we will use the dual variational approach introduced in 31 and consider the operator $K_{Q}$ formally defined as $K_{Q} f=Q^{\frac{1}{p}} R\left(Q^{\frac{1}{p}} f\right)$, where $R$ denotes the real part of the Helmholtz resolvent operator $\mathcal{R}$, i.e., $R g=(\operatorname{Re} \Phi) * g$ with the fundamental solution $\Phi$ defined in 3.3 .

To analyze the mapping properties of $K_{Q}$ and to set up a variational framework, we assume, as in Theorem 3.5. that $q \in\left[1, \frac{2(N+1)}{N-1}\right]$ and $p \in\left(\max \left\{\frac{2 N}{N-1} \frac{2 q}{q+2}, 2\right\}, \frac{2 N}{N-2}\right)$ are fixed such that $\left(G, q, Q^{\frac{1}{p}}\right)$ is an admissible extension triple. The following is an immediate consequence of Theorem 3.12 . 
Lemma 3.21. Let $\tilde{p}, r \in(1, \infty)$ satisfy (3.31) with $\tilde{p}$ in place of $p$, and suppose moreover that (3.32) holds with $\tilde{p}$ in place of $p$ if $q \geq 2$, and that (3.33) holds with $\tilde{p}$ in place of $p$ if $q<2$. Then the operator $K_{Q}$ is bounded as a map $L_{G}^{\tilde{p}^{\prime}}\left(\mathbb{R}^{N}\right) \rightarrow L_{G}^{r}\left(\mathbb{R}^{N}\right)$.

We note that Lemma 3.21 applies in particular in the case $r=\tilde{p}=p$, so $K_{Q}$ is a bounded operator $L_{G}^{p^{\prime}}\left(\mathbb{R}^{N}\right) \rightarrow L_{G}^{p}\left(\mathbb{R}^{N}\right)$.

We also note the following immediate corollary of Lemma 3.21 .

Corollary 3.22. There exist $\sigma_{1}<p<\sigma_{2}$ with the property that $K_{Q}$ is bounded as a map $L_{G}^{\sigma_{i}^{\prime}}\left(\mathbb{R}^{N}\right) \rightarrow L_{G}^{p}\left(\mathbb{R}^{N}\right)$ and as a map $L_{G}^{p^{\prime}}\left(\mathbb{R}^{N}\right) \rightarrow L_{G}^{\sigma_{i}}\left(\mathbb{R}^{N}\right)$ for $i=1,2$.

Next we note the following variant of [31, Lemma 4.1].

LEMmA 3.23. The operator $K_{Q}: L_{G}^{p^{\prime}}\left(\mathbb{R}^{N}\right) \rightarrow L_{G}^{p}\left(\mathbb{R}^{N}\right)$ is locally compact, i.e., the operators

$$
K_{Q} \mathbb{1}_{B}: L_{G}^{p^{\prime}}\left(\mathbb{R}^{N}\right) \rightarrow L_{G}^{p}\left(\mathbb{R}^{N}\right) \quad \text { and } \quad \mathbb{1}_{B} K_{Q}: L_{G}^{p^{\prime}}\left(\mathbb{R}^{N}\right) \rightarrow L_{G}^{p}\left(\mathbb{R}^{N}\right)
$$

are compact for every bounded and measurable set $B \subset \mathbb{R}^{N}$.

Proof. Let $B \subset \mathbb{R}^{N}$ be bounded and measurable, and fix $s \in\left[\frac{2(N+1)}{N-1}, \frac{2 N}{N-2}\right)$ with $s \geq p$, i.e., $s^{\prime} \leq p^{\prime}$. By [31, Lemma 4.1], the operator $\mathbb{1}_{B} K_{Q}: L^{s^{\prime}}\left(\mathbb{R}^{N}\right) \rightarrow L^{s}\left(\mathbb{R}^{N}\right)$ is compact. By duality, the operator $K_{Q} \mathbb{1}_{B}: L^{s^{\prime}}\left(\mathbb{R}^{N}\right) \rightarrow L^{s}\left(\mathbb{R}^{N}\right)$ is therefore also compact.

Next, let $\left(v_{n}\right)_{n} \subset L_{G}^{p^{\prime}}\left(\mathbb{R}^{N}\right)$ be a sequence with $v_{n} \rightarrow 0$ in $L_{G}^{p^{\prime}}\left(\mathbb{R}^{N}\right)$. Then we have $w_{n}:=\mathbb{1}_{B} v_{n} \rightarrow$ 0 in $L_{G}^{p^{\prime}}\left(\mathbb{R}^{N}\right)$, and thus also in $L_{G}^{s^{\prime}}\left(\mathbb{R}^{N}\right)$, since $B$ has finite measure. By the compactness property mentioned above, it follows that $K_{Q} \mathbb{1}_{B} v_{n}=K_{Q} \mathbb{1}_{B} w_{n} \rightarrow 0$ strongly in $L_{G}^{s}\left(\mathbb{R}^{N}\right)$. Moreover, it follows from Corollary 3.22 that the sequence of functions $K_{Q} \mathbb{1}_{B} v_{n}=K_{Q} w_{n}$, $n \in \mathbb{N}$ is bounded in $L_{G}^{\sigma_{1}}\left(\mathbb{R}^{N}\right)$ for some $\sigma_{1}<p$. Since $\sigma_{1}<p \leq s$, it thus follows by interpolation that there exists $\theta \in(0,1]$ with

$$
\left\|K_{Q} \mathbb{1}_{B} v_{n}\right\|_{p} \leq\left\|K_{Q} \mathbb{1}_{B} v_{n}\right\|_{\sigma_{1}}^{1-\theta}\left\|K_{Q} \mathbb{1}_{B} v_{n}\right\|_{s}^{\theta} \rightarrow 0 \quad \text { as } n \rightarrow \infty .
$$

Hence the operator $K_{Q} \mathbb{1}_{B}: L_{G}^{p^{\prime}}\left(\mathbb{R}^{N}\right) \rightarrow L_{G}^{p}\left(\mathbb{R}^{N}\right)$ is compact, and by duality it follows that also $\mathbb{1}_{B} K_{Q}: L_{G}^{p^{\prime}}\left(\mathbb{R}^{N}\right) \rightarrow L_{G}^{p}\left(\mathbb{R}^{N}\right)$ is a compact operator.

As in [31], we now introduce the (dual) energy functional

$$
J: L_{G}^{p^{\prime}}\left(\mathbb{R}^{N}\right) \rightarrow \mathbb{R}, \quad J(v)=\frac{1}{p^{\prime}} \int_{\mathbb{R}^{N}}|v|^{p^{\prime}} d x-\frac{1}{2} \int_{\mathbb{R}^{N}} v\left[K_{Q} v\right](x) d x .
$$

Then $J$ is of class $\mathcal{C}^{1}$ with

$$
J^{\prime}(v) w=\int_{\mathbb{R}^{N}}\left(|v|^{p^{\prime}-2} v-K_{Q} v\right) w d x \quad \text { for } v, w \in L_{G}^{p^{\prime}}\left(\mathbb{R}^{N}\right) .
$$

Moreover, we have 
LEMMA 3.24. If $v \in L_{G}^{p^{\prime}}\left(\mathbb{R}^{N}\right)$ is a critical point of $J$, then $u=R Q^{\frac{1}{p}} v$ is a real-valued solution of (3.54) of class $W^{2, q}\left(\mathbb{R}^{N}\right) \cap \mathcal{C}^{1, s}\left(\mathbb{R}^{N}\right)$ for $q \geq p, s \in(0,1)$.

Proof. Let $w \in L^{p^{\prime}}\left(\mathbb{R}^{N}\right)$, and let $w_{G} \in L^{p^{\prime}}\left(\mathbb{R}^{N}\right)$ be defined by

$$
w_{G}=\int_{G} w \circ \operatorname{Ad\mu }(A), \quad \text { i.e., } \quad w_{G}(x)=\int_{G} w(A x) d \mu(A) \quad \text { for } x \in \mathbb{R}^{N},
$$

where $\mu$ is the Haar-measure of $G$. Since $v$ is $G$-invariant, it follows that

$$
\int_{\mathbb{R}^{N}}\left(|v|^{p^{\prime}-2} v-K_{Q} v\right) w d x=\int_{\mathbb{R}^{N}}\left(|v|^{p^{\prime}-2} v-K_{Q} v\right)[w \circ A] d x \quad \text { for all } A \in G
$$

and therefore

$$
\int_{\mathbb{R}^{N}}\left(|v|^{p^{\prime}-2} v-K_{Q} v\right) w d x=\int_{\mathbb{R}^{N}}\left(|v|^{p^{\prime}-2} v-K_{Q} v\right) w_{G} d x=J^{\prime}(v) w_{G}=0
$$

Consequently, we have $|v|^{p^{\prime}-2} v=K_{Q} v$ in $L^{p}\left(\mathbb{R}^{N}\right)$, which implies that $u=R Q^{\frac{1}{p}} v$ satisfies the equation

$$
u=R Q|u|^{p-2} u \quad \text { in } L^{p}\left(\mathbb{R}^{N}\right) .
$$

The claim now follows by [31, Lemma 4.3].

Next we note that the functional $J$ has a mountain pass geometry. More precisely, we have:

Lemma 3.25. (i) There exists $\delta>0$ and $0<\rho<1$ such that $J(v) \geq \delta>0$ for all $v \in L_{G}^{p^{\prime}}\left(\mathbb{R}^{N}\right)$ with $\|v\|_{p^{\prime}}=\rho$.

(ii) There is $v_{0} \in L_{G}^{p^{\prime}}\left(\mathbb{R}^{N}\right)$ such that $\left\|v_{0}\right\|_{p^{\prime}}>1$ and $J\left(v_{0}\right)<0$.

(iii) Every Palais-Smale sequence for $J$ is bounded in $L_{G}^{p^{\prime}}\left(\mathbb{R}^{N}\right)$.

(iv) There exists a Palais-Smale sequence for $J$ at the mountain pass level

$$
d:=\inf _{\gamma \in \Gamma} \max _{t \in[0,1]} J(\gamma(t))>0,
$$

where $\Gamma=\left\{\gamma \in C\left([0,1], L_{G}^{p^{\prime}}\left(\mathbb{R}^{N}\right)\right): \gamma(0)=0,\|\gamma(1)\|_{p^{\prime}}>\rho, J(\gamma(1))<0\right\}$.

Proof. Since $p>2$, the parts (i)-(iii) are proved in [31, Lemma 4.2] for $G=\{i d\}$, and the proof remains the same for general closed subgroups $G \subset O(N)$. Moreover, the positivity of the mountain pass level $c$ defined in (3.55) is a direct consequence of (i) and (ii), which also shows that the set $\Gamma$ is nonempty. Finally, the proof of the existence of a Palais-Smale sequence for $J$ at level $d$ is exactly the same as the proof of [31, Lemma 6.1]. Here we note that periodicity of $Q$ was assumed in [31, Section 6], but this property is not used in Lemma 6.1 .

Proposition 3.26. Let $\left(v_{n}\right)_{n} \subset L_{G}^{p^{\prime}}\left(\mathbb{R}^{N}\right)$ be a Palais-Smale sequence of $J$ with $c:=\lim _{n \rightarrow \infty} J\left(v_{n}\right)>$ 0. Moreover, suppose that one of the following conditions hold: 
(A1) For some $R>0$, we have $\lim _{|x| \rightarrow \infty}\|Q\|_{L^{1}\left(B_{R}(x)\right)}=0$.

(A2) For every $R>0$ we have $\lim _{|x| \rightarrow \infty} N_{G}(x, R)=\infty$, where, for $R>0$ and $x \in \mathbb{R}^{N} \backslash\{0\}$, $N_{G}(x, R)$ denotes the maximal number of elements of a subset $H \subset G$ with $B_{R}(A x) \cap$ $B_{R}\left(A^{\prime} x\right)=\varnothing$ for $A, A^{\prime} \in H$.

Then, after passing to a subsequence, we have

$$
v_{n} \rightarrow v \quad \text { in } L^{p^{\prime}}\left(\mathbb{R}^{N}\right)
$$

where $v \in L_{G}^{p^{\prime}}\left(\mathbb{R}^{N}\right) \backslash\{0\}$ is a critical point of $J$.

Proof. We first note that $\left(v_{n}\right)_{n}$ is bounded by Lemma 3.25 . Consequently, since $L_{G}^{p^{\prime}}\left(\mathbb{R}^{N}\right)$ is reflexive, there exists $v \in L_{G}^{p^{\prime}}\left(\mathbb{R}^{N}\right)$ such that

$$
v_{n} \rightarrow v \quad \text { in } L^{p^{\prime}}\left(\mathbb{R}^{N}\right) .
$$

Moreover,

$$
\lim _{n \rightarrow \infty} \int_{\mathbb{R}^{N}} v_{n} K_{Q} v_{n} d x=\frac{2 p^{\prime}}{2-p^{\prime}} \lim _{n \rightarrow \infty}\left(J\left(v_{n}\right)-\frac{1}{p^{\prime}} J^{\prime}\left(v_{n}\right) v_{n}\right)=\frac{2 p^{\prime}}{2-p^{\prime}} c>0
$$

by assumption, which implies that

$$
\lim _{n \rightarrow \infty}\left|\int_{\mathbb{R}^{N}} v_{n} \mathcal{R}_{Q^{\frac{1}{p}}} v_{n} d x\right|>0
$$

Since moreover $\left(G, q, Q^{\frac{1}{p}}\right)$ is an admissible extension triple by assumption, Theorem 3.4 applies and yields $\delta, R>0$ and a sequence of points $\left(x_{n}\right)_{n} \subset \mathbb{R}^{N}$ such that, after passing to a subsequence,

$$
\int_{B_{R}\left(x_{n}\right)}\left|Q^{\frac{1}{p}} v_{n}\right|^{p^{\prime}} d x \geq \delta>0, \quad \text { for all } n \in \mathbb{N} .
$$

We claim that $\left(x_{n}\right)_{n}$ has to be bounded. To see this, we argue by contradiction and assume that, after passing to a subsequence again, $\left|x_{n}\right| \rightarrow \infty$. We distinguish two cases.

\section{Case 1: (A1) holds.}

In this case we put $\varphi_{n}:=Q^{p-1} v_{n} \mathbb{1}_{B_{R}\left(x_{n}\right)}$, and we note that $\left(\varphi_{n}\right)_{n}$ is a bounded sequence in $L^{p^{\prime}}\left(\mathbb{R}^{N}\right)$. Moreover, we have

$$
\begin{aligned}
\int_{B_{R}\left(x_{n}\right)}\left|Q^{\frac{1}{p}} v_{n}\right|^{p^{\prime}} d x & =\int_{\mathbb{R}^{N}}\left|v_{n}\right|^{p^{\prime}-2} v_{n} \varphi_{n} d x=J^{\prime}\left(v_{n}\right) \varphi_{n}+\int_{\mathbb{R}^{N}} v_{n} K_{Q} \varphi_{n} d x \\
& \leq o(1)\left\|\varphi_{n}\right\|_{p}+\left|\int_{\mathbb{R}^{N}} v_{n} K_{Q} \varphi_{n} d x\right|=o(1)+\left\|v_{n}\right\|_{p^{\prime}}\left\|K_{Q} \varphi_{n}\right\|_{p}
\end{aligned}
$$


as $n \rightarrow \infty$. By Corollary 3.22 , there exists $\sigma>p^{\prime}$ and $C>0$ with the property that

$$
\left\|K_{Q} \varphi_{n}\right\|_{p} \leq C\left\|\varphi_{n}\right\|_{\sigma^{\prime}} \quad \text { for } n \in \mathbb{N},
$$

whereas, since $\sigma^{\prime}<p^{\prime}$ and by Hölder's inequality,

$$
\begin{aligned}
\left\|\varphi_{n}\right\|_{\sigma^{\prime}} & =\left\|Q^{p-1} v_{n}\right\|_{L^{\sigma^{\prime}}\left(B_{R}\left(x_{n}\right)\right)} \leq\left(\int_{B_{R}\left(x_{n}\right)}|Q|^{\frac{p^{\prime} \sigma^{\prime}(p-1)}{p^{\prime}-\sigma^{\prime}}} d x\right)^{\frac{p^{\prime}-\sigma^{\prime}}{p^{\prime} \sigma^{\prime}}}\left\|v_{n}\right\|_{p^{\prime}} \\
& \leq\left(\|Q\|_{L^{1}\left(B_{R}\left(x_{n}\right)\right)}\right)^{\frac{p^{\prime}-\sigma^{\prime}}{p^{\prime} \sigma^{\prime}}}\|Q\|_{\infty}\left(\frac{p^{\prime} \sigma^{\prime}(p-1)}{p^{\prime}-\sigma^{\prime}}-1\right) \frac{p^{\prime}-\sigma^{\prime}}{p^{\prime} \sigma^{\prime}}\left\|v_{n}\right\|_{p^{\prime}}
\end{aligned}
$$

Since $\|Q\|_{L^{1}\left(B_{R}\left(x_{n}\right)\right)} \rightarrow 0$ by $(3.10)$, it thus follows that $\left\|\varphi_{n}\right\|_{\sigma^{\prime}} \rightarrow 0$ as $n \rightarrow \infty$. Here we note that, by an easy covering argument, (3.10) holds for every $R>0$ if it holds for one $R>0$. Going back to (3.58), we thus deduce that

$$
\int_{B_{R}\left(x_{n}\right)}\left|Q^{\frac{1}{p}} v_{n}\right|^{p^{\prime}} d x \rightarrow 0 \quad \text { as } n \rightarrow \infty,
$$

which contradicts 3.57).

\section{Case 2: (A2) holds.}

In this case it follows from (3.57) and the fact that $v_{n}$ and $Q$ are $G$-invariant that

$$
\left\|Q^{\frac{1}{p}} v_{n}\right\|_{p^{\prime}}^{p^{\prime}} \geq N_{G}\left(x_{n}, R\right) \int_{B_{R}\left(x_{n}\right)}\left|Q^{\frac{1}{p}} v_{n}\right|^{p^{\prime}} d x \geq N_{G}\left(x_{n}, R\right) \delta \rightarrow \infty
$$

as $n \rightarrow \infty$, which contradicts the boundedness of the sequence $\left(v_{n}\right)_{n}$ in $L^{p^{\prime}}\left(\mathbb{R}^{N}\right)$.

Since in both cases we have reached a contradiction, we conclude that $\left(x_{n}\right)_{n}$ is bounded. Therefore, making $R$ larger if necessary, we can assume that (3.57) holds with $x_{n}=0$ for all $n \in \mathbb{N}$. Now for any fixed $G$-invariant function $\varphi \in \mathcal{C}_{c}^{\infty}\left(\mathbb{R}^{N}\right)$, any $r>0$ and $n, m \in \mathbb{N}$ we have

$$
\begin{aligned}
\left|\int_{\mathbb{R}^{N}}\left(\left|v_{n}\right|^{p^{\prime}-2} v_{n}-\left|v_{m}\right|^{p^{\prime}-2} v_{m}\right) \varphi d x\right| & =\left|J^{\prime}\left(v_{n}\right) \varphi-J^{\prime}\left(v_{m}\right) \varphi+\int_{B_{r}} \varphi K_{Q}\left(v_{n}-v\right) d x\right| \\
& \leq\left\|J^{\prime}\left(v_{n}\right)-J^{\prime}\left(v_{m}\right)\right\|\|\varphi\|_{p^{\prime}}+\left\|\mathbb{1}_{B_{r}} K_{Q}\left(v_{n}-v_{m}\right)\right\|_{p}\|\varphi\|_{p^{\prime}} .
\end{aligned}
$$

So by assumption and the local compactness of $K_{Q}$, as stated in Lemma 3.23, we get that $\left(\left|v_{n}\right|^{p^{\prime}-2} v_{n}\right)_{n \in \mathbb{N}}$ is a Cauchy sequence in $L^{p}\left(B_{R}\right)$. Consequently, $\left|v_{n}\right|^{p^{\prime}-2} v_{n} \rightarrow \tilde{v}$ strongly in $L^{p}\left(B_{R}\right)$ for some $\tilde{v} \in L^{p}\left(B_{R}\right)$, and passing to a subsequence also pointwisely almost everywhere on $B_{R}$. This clearly implies that $v_{n} \rightarrow|\tilde{v}|^{p-2} \tilde{v}$ almost everywhere on $B_{r}$. Now 3.56 and the uniqueness of the weak limit gives $\tilde{v}=|v|^{p^{\prime}-2} v$ and

$$
0<\delta \leq \int_{B_{R}}\left|Q^{\frac{1}{p}} v_{n}(x)\right|^{p^{\prime}} d x \rightarrow \int_{B_{R}}\left|Q^{\frac{1}{p}} v\right|^{p^{\prime}} d x, \quad \text { as } n \rightarrow \infty
$$


which implies that $v \neq 0$.

For every $G$-invariant function $\varphi \in \mathcal{C}_{c}^{\infty}$, we now have

$$
\begin{aligned}
J^{\prime}(v) \varphi & =\int_{\mathbb{R}^{N}}|v|^{p^{\prime}-2} v \varphi d x-\int_{\mathbb{R}^{N}} \varphi K_{Q}(v) d x \\
& =\lim _{n \rightarrow \infty}\left[\int_{\mathbb{R}^{N}}\left|v_{n}\right|^{p^{\prime}-2} v_{n} \varphi d x-\int_{\mathbb{R}^{N}} \varphi K_{Q}\left(v_{n}\right) d x\right] \\
& =\lim _{n \rightarrow \infty} J^{\prime}\left(v_{n}\right) \varphi=0
\end{aligned}
$$

using the local strong convergence of $\left|v_{n}\right|^{p^{\prime}-2} v_{n}$ and the continuity of linear operator $K_{Q}$ : $L_{G}^{p^{\prime}}\left(\mathbb{R}^{N}\right) \rightarrow L_{G}^{p}\left(\mathbb{R}^{N}\right)$. By density, it now follows that $J^{\prime}(v) w=0$ for every $w \in L_{G}^{p^{\prime}}\left(\mathbb{R}^{N}\right)$, i.e., $v \in L_{G}^{p^{\prime}}\left(\mathbb{R}^{N}\right) \backslash\{0\}$ is a critical point of $J$.

We now have all the tools to complete the proofs of our main existence results for nontrivial $G$-invariant dual ground state solutions as stated in the introduction.

Proof of Theorem 3.5 (completed). By Lemma 3.25(iv), there exists a Palais-Smale sequence $\left(v_{n}\right)_{n}$ in $L_{G}^{p^{\prime}}\left(\mathbb{R}^{N}\right)$ for $J$ at the mountain pass level $d>0$. By Proposition 3.26, we have $v_{n} \rightarrow v$ in $L_{G}^{p^{\prime}}\left(\mathbb{R}^{N}\right)$ after passing to a subsequence, where $v \in L_{G}^{p^{\prime}}\left(\mathbb{R}^{N}\right)$ is a nontrivial critical point of $J$. Here we note that assumption (A1) of Proposition 3.26 is satisfied by 3.10$)$. The proof is finished by Lemma 3.24 .

Proof of Corollary 3.6. Since $Q^{\frac{1}{p}} \in L^{\infty}\left(\mathbb{R}^{N}\right)$, it follows by the classical Stein-Tomas estimate that $\left(G, q, Q^{\frac{1}{p}}\right)$ is an admissible extension triple for $q=\frac{2(N+1)}{N-1}$. Since

$$
p \in\left(\frac{2(N+1)}{N-1}, \frac{2 N}{N-2}\right)=\left(\frac{2 N}{N-1} \frac{2 q}{q+2}, \frac{2 N}{N-2}\right),
$$

the assumptions of Theorem 3.5 are satisfied and yield the existence of a nontrivial solution $v \in L_{G}^{p^{\prime}}\left(\mathbb{R}^{N}\right)$ of 3.4 .

Proof of Theorem 3.7. As above, it follows by the classical Stein-Tomas estimate that $\left(G_{k}, q, Q^{\frac{1}{p}}\right)$ is an admissible extension triple for $q=\frac{2(N+1)}{N-1}$, whereas

$$
p \in\left(\frac{2(N+1)}{N-1}, \frac{2 N}{N-2}\right)=\left(\frac{2 N}{N-1} \frac{2 q}{q+2}, \frac{2 N}{N-2}\right) .
$$

Moreover, since $2 \leq k \leq N-2$, we have

$$
\lim _{|x| \rightarrow \infty} N_{G}(x, R)=\infty \quad \text { for every } R>0
$$

where $N_{G}(x, R)$ is defined as in Proposition 3.26 . This fact is noted without proof in [70, Proof of Corollary 1.25], and we give the short proof here for the reader's convenience. In fact, 3.59) follows already from the fact that the minimal orbit dimension of $G_{k}$ is $\min \{k-1, N-k-1\}$ and therefore greater than or equal to one by assumption. In particular, for every $n \in \mathbb{N}$ and 
$\theta \in \mathbb{S}^{N-1}$, there exists $\varepsilon>0$ and a subset $H_{\theta} \subset G$ with $B_{\varepsilon}(A \theta) \cap B_{\varepsilon}\left(A^{\prime} \theta\right)=\varnothing$ for every $A, A^{\prime} \in H_{\theta}$. Moreover, by a straightforward compactness argument, $\varepsilon>0$ can be chosen to depend only on $n$ and not on $\theta \in \mathbb{S}^{N-1}$. Hence, if $R>0$ is given, $x \in \mathbb{R}^{N}$ satisfies $|x| \geq \frac{R}{\varepsilon}$ and $\theta$ equals $\frac{x}{|x|}$, we have $B_{R}(A \theta) \cap B_{R}\left(A^{\prime} \theta\right)=\varnothing$ for every $A, A^{\prime} \in H_{\theta}$ and therefore $N_{G}(x, R) \geq n$. This shows (3.59).

Hence assumption (A2) of Proposition 3.26 is satisfied, and thus the proof is completed as the proof of Theorem 3.5 above.

Proof of Corollary 3.8. We first note that $Q$ satisfies the asymptotic condition (3.10). Indeed, since $0 \leq Q \leq c \mathbb{1}_{L_{\alpha}}$ for some $c>0$ by assumption, it suffices to show that

$$
\left|L_{\alpha} \cap B_{R}(x)\right| \rightarrow 0 \quad \text { as }|x| \rightarrow \infty \text { for every } R>0 .
$$

To see the latter, it suffices to consider a sequence $\left(x_{n}\right)_{n}=\left(x_{n}^{(N-k)}, x_{n}^{(k)}\right) \subset \mathbb{R}^{N-k} \times \mathbb{R}^{k}$ with $x_{n}^{(N-k)}=0$ for all $n \in \mathbb{N}$ and $r_{n}:=\left|x_{n}\right|=\left|x_{n}^{(k)}\right| \rightarrow \infty$ as $n \rightarrow \infty$. In this case we have $\left|x^{(k)}-x_{n}^{(k)}\right|<R$ for $x \in B_{R}\left(x_{n}\right)$ and therefore

$$
\begin{aligned}
\left|L_{\alpha} \cap B_{R}\left(x_{n}\right)\right| \leq & \iint_{\left\{\left|x^{(k)}-x_{n}^{(k)}\right|<R\right\}} d x^{(N-k)} d x^{(k)} \leq C \int_{\left\{\left|x^{(k)}-x_{n}^{(k)}\right|<R\right\}}\left|x^{(k)}\right|^{-(N-k) \alpha} d x^{(k)} \\
& =C \int_{\left|z^{(k)}\right|<R}\left|z^{(k)}+x_{n}^{(k)}\right|^{-(N-k) \alpha} d x^{(k)} \leq C \int_{\left|z^{(k)}\right|<R}\left(\left|x_{n}^{(k)}\right|-R\right)^{-(N-k) \alpha} d x^{(k)} \\
& =C\left(r_{n}-R\right)^{-(N-k) \alpha} \rightarrow 0 \quad \text { as } n \rightarrow \infty
\end{aligned}
$$

with constants $C>0$. Hence 3.60 holds.

Next, we first consider the case $k=1$. By Theorem 3.2 additionally the condition $\alpha>\frac{1}{N-1}$ is required and we set $\lambda=\frac{2(N-1)-\frac{2}{\alpha}}{N-2}$. By case distinction we see that $\mu_{N, 1, \alpha}=\max \left\{\frac{2 N}{N-1} \frac{2 \lambda}{\lambda+2}, 2\right\}$ for $\alpha>\frac{1}{N-1}$. Thus by Theorem 3.2 we may fix any $q \in\left(\mu_{N, 1, \alpha}, p\right)$ with $p \in\left(\mu_{N, 1, \alpha}, \frac{2 N}{N-2}\right)$ such that $\left(G_{1}, q, \mathbb{1}_{L_{\alpha}}\right)$ is an admissible extension triple. Since $0 \leq Q^{\frac{1}{p}} \leq c^{\frac{1}{p}} \mathbb{1}_{L_{\alpha}}$, it follows that also $\left(G_{1}, q, Q^{\frac{1}{p}}\right)$ is an admissible extension triple. Thus Theorem 3.5 applies and yields that (3.4) admits a dual bound state solution.

The case $k=N-1$ now follows similarly: Consider additionally $\alpha<N-1$, set $\lambda=\frac{2(N-1)-2 \alpha}{N-2}$ and observe that for $\alpha<N-1$ the expression $\mu_{N, N-1, \alpha}$ is chosen such that $\mu_{N, N-1, \alpha}=$ $\max \left\{\frac{2 N}{N-1} \frac{2 \lambda}{\lambda+2}, 2\right\}$. Then, for $q, p$ as above with $\mu_{N, N-1, \alpha}$ instead of $\mu_{N, 1, \alpha}$ we conclude that $\left(G_{N-1}, q, Q^{\frac{1}{p}}\right)$ is admissible extension triple and Theorem 3.5 again yields the existence of a dual bound state solution of (3.4).

If $2 \leq k \leq N-2$ and $p \in\left(\mu_{N, k, \alpha}, \frac{2 N}{N-2}\right)$ again a case distinction shows that $\mu_{N, k, \alpha}=$ $\max \left\{\frac{2 N}{N-1} \frac{2 \lambda}{\lambda+2}, 2\right\}$, where $\lambda:=\lambda_{N, k, \alpha}$ is given in Theorem 3.2. Consequently, by Theorem 3.2 . we may fix $q \in\left(\mu_{N, k, \alpha}, p\right)$ with $\max \left\{\frac{2 N}{N-1} \frac{2 q}{q+2}, 2\right\}<p<\frac{2 N}{N-2}$ and the property that $\left(G_{k}, q, \mathbb{1}_{L_{\alpha}}\right)$ is an admissible extension triple. As above, it follows that also $\left(G_{k}, q, Q^{\frac{1}{p}}\right)$ is an admissible 
extension triple. Again, Theorem 3.5 applies and yields that (3.4) admits a nontrivial dual bound state solution. Thus the claim holds in this case as well.

REMARK 3.27. We note that Corollary 3.8 extends to the case where $L_{\alpha}$ is replaced by the more general class of sets $L_{\alpha, \beta}$ considered in Theorem 3.10. For this, one has to additionally assume $\beta>\frac{1}{N-1}$ if $k=1$. Then the statement of Corollary 3.8 holds with $\mu_{N, 1, \beta}$. If $k=N-1$ the statement holds with the same value $\mu_{N, N-1, \alpha}$. For $2 \leq k \leq N-2$ the value $\mu_{N, k, \alpha}$ needs to be replaced by $\max \left\{\frac{2 N}{N-1} \frac{2 \lambda}{\lambda+2}, 2\right\}$, where now $\lambda=\lambda_{N, k, \alpha, \beta}$ is given in 3.18) 


\section{CHAPTER 4}

\section{Dual variational methods for a nonlinear Helmholtz equation with sign-changing nonlinearity}

In this chapter we will present how sign-changing nonlinearities can be incorporated into the dual variational framework for the nonlinear Helmholtz equation and establish the existence of real valued solutions in this context. The presented article [Y3] resulted as a collaboration with Rainer Mandel and Dominic Scheider. The presentation is in the very same form as the publication [Y3], with the exception that the acknowledgement is excluded. The notation may slightly differ from the notation in the previous chapters.

\section{Introduction}

In the present article, we consider nonlinear Helmholtz equations of the form

$$
-\Delta u-k^{2} u=Q(x)|u|^{p-2} u \quad \text { on } \mathbb{R}^{N}
$$

for $p \in\left[\frac{2(N+1)}{N-1}, \frac{2 N}{N-2}\right)$ and $k>0$ with a weight function $Q \in L^{\infty}\left(\mathbb{R}^{N}\right)$ that may change sign. To allow for the latter is nontrivial given that one of the main tools for proving the existence of solutions is the dual variational method that, in its classical form, relies on the nonnegativity of the weight function. In the context of Nonlinear Helmholtz equations it was first implemented in a paper by Evéquoz and Weth [31. To highlight the role of the nonnegativity of $Q$ we briefly recapitulate the approach.

Instead of 4.1 one considers a reformulation as the integral equation

$$
u=\mathbf{R}\left(Q|u|^{p-2} u\right) \quad u \in L^{p}\left(\mathbb{R}^{N}\right),
$$

where $\mathbf{R}$ is the real part of a resolvent type operator $\mathcal{R}$, i.e., a right inverse of the Helmholtz operator $-\Delta-k^{2}$ on $\mathbb{R}^{N}$. For $f \in \mathcal{S}\left(\mathbb{R}^{N}\right)$ the operator $\mathcal{R}$ is given by $\mathcal{R}(f)=\Phi * f$ where

$$
\Phi(x):=\frac{\mathrm{i}}{4}\left(\frac{k}{2 \pi|x|}\right)^{\frac{N-2}{2}} H_{\frac{N-2}{2}}^{(1)}(k|x|), \quad x \in \mathbb{R}^{N} \backslash\{0\}
$$

is the fundamental solution of the Helmholtz equation associated with Sommerfeld's outgoing radiation condition

$$
\left|\nabla \Phi(x)-k \mathrm{i} \Phi(x) \frac{x}{|x|}\right|=o\left(|x|^{\frac{1-N}{2}}\right), \quad \text { as }|x| \rightarrow \infty
$$


Here, $H_{\frac{N-2}{2}}^{(1)}$ denotes the Hankel function of the first kind and order $\frac{N-2}{2}$. So the operator $\mathbf{R}$ from 4.2 is given by $\mathbf{R}(f)=\Psi * f$ where $\Psi:=\operatorname{Re}(\Phi)$ is given by

$$
\Psi(x)=-\frac{1}{4}\left(\frac{k}{2 \pi|x|}\right)^{\frac{N-2}{2}} Y_{\frac{2-N}{2}}(k|x|), \quad x \in \mathbb{R}^{N} \backslash\{0\}
$$

It is known [43, Theorem 2.3] that $\mathcal{R}$ extends as a continuous linear map from $L^{p^{\prime}}\left(\mathbb{R}^{N}\right) \rightarrow$ $L^{p}\left(\mathbb{R}^{N}\right)$ precisely for $p \in\left[\frac{2(N+1)}{N-1}, \frac{2 N}{N-2}\right]$. One then introduces the dual variable $\tilde{u}:=Q^{1 / p^{\prime}}|u|^{p-2} u$ and observes that solutions of 4.2 are precisely the critical points of the (dual) energy functional $I: L^{p^{\prime}}\left(\mathbb{R}^{N}\right) \rightarrow L^{p}\left(\mathbb{R}^{N}\right)$ given by

$$
I(\tilde{u}):=\frac{1}{p^{\prime}}\|\tilde{u}\|_{p^{\prime}}^{p^{\prime}}-\frac{1}{2} \int_{\mathbb{R}^{N}} \tilde{u} \mathcal{K} \tilde{u} d x .
$$

Here, $\mathcal{K}: L^{p^{\prime}}\left(\mathbb{R}^{N}\right) \rightarrow L^{p}\left(\mathbb{R}^{N}\right), \tilde{u} \mapsto Q^{\frac{1}{p}} \mathbf{R}\left(Q^{\frac{1}{p}} \tilde{u}\right)$ is a symmetric operator in the sense of

$$
\int_{\mathbb{R}^{N}} f \mathcal{K} g d x=\int_{\mathbb{R}^{N}} g \mathcal{K} f d x \quad \text { for all } f, g \in L^{p^{\prime}}\left(\mathbb{R}^{N}\right),
$$

see [31, Lemma 4.1]. Under the additional assumption that $Q$ vanishes at infinity, one obtains that $I$ is an odd functional of class $\mathcal{C}^{1}$ that has the Mountain Pass Geometry and satisfies the Palais-Smale Condition. So the existence of an unbounded sequence of solutions to (4.2) follows from the Symmetric Mountain Pass Theorem. Inverting the transformation $u \mapsto \tilde{u}$ one thus obtains an unbounded sequence of solutions to the nonlinear Helmholtz equation (4.1). This is the strategy proposed by Evéquoz and Weth [31] for the focusing nonlinear Helmholtz equation $Q \geq 0$. We refer to [52 for analogous results in the defocusing case $Q \leq 0$, where the dual variational approach was implemented for the dual variable $\tilde{u}:=|Q|^{1 / p^{\prime}}|u|^{p-2} u$. In view of these two results it is natural to ask for a dual variational approach work in the intermediate case of sign-changing $Q$. In this paper, we provide a solution for this problem.

To treat sign-changing coefficients $Q \in L^{\infty}\left(\mathbb{R}^{N}\right)$ we have to come up with a new idea to make the dual variation approach work. We write $Q=Q_{+}-Q_{-}$where $Q_{ \pm}:=|Q| \mathbb{1}_{A_{ \pm}}$and

$$
A_{+}:=\{Q>0\}, \quad A_{-}:=\{Q \leq 0\} .
$$

In fact we will consider $Q_{\lambda}:=\lambda Q_{+}-Q_{-}$for $\lambda>0$ in the following. Our main idea is to introduce a tuple of dual variables $(\varphi, \psi) \in L^{p^{\prime}}\left(A_{+}\right) \times L^{p^{\prime}}\left(A_{-}\right)$associated with $\left(\left.u\right|_{A_{+}},\left.u\right|_{A_{-}}\right)$ and to derive a coupled system of nonlinear integral equations the solutions of which are precisely the critical points of an associated strongly indefinite dual energy functional. We will see that the indefiniteness comes from the presence of $Q_{-}$and thus vanishes in the case of a nonnegative function $Q \geq 0$. In particular, the critical points of this dual energy functional will have infinite Morse index, which clearly distinguishes these solutions from the dual bound 
and ground states obtained in $[31$ in the case $Q \geq 0$. We will explain the dual variational framework in detail in Section 2 .

Our conditions for the existence of critical points involve the linear operator $\mathcal{K}: L^{p^{\prime}}\left(\mathbb{R}^{N}\right) \rightarrow$ $L^{p}\left(\mathbb{R}^{N}\right), f \mapsto|Q|^{\frac{1}{p}} \mathbf{R}\left(|Q|^{\frac{1}{p}} f\right)$ as well as the numbers

$$
\alpha:=\max _{\substack{\|\varphi\|_{p^{\prime}=1,} \\ \operatorname{supp}(\varphi) \subset A_{+} \mathbb{R}^{N}}} \int_{\mathcal{K}} \varphi d x, \quad \beta:=\max _{\substack{\|\varphi\|_{p^{\prime}=\|\psi\|_{p^{\prime}}=1,} \\ \operatorname{supp}(\varphi) \subset A_{+}, \operatorname{supp}(\psi) \subset A_{-}}} \int_{\mathbb{R}^{N}} \varphi \mathcal{K} \psi d x .
$$

Since we will assume $\mathcal{K}$ to be compact, both values are indeed attained. Moreover we have $\beta \geq 0$ and [31, Lemma 4.2(ii)] gives $\alpha>0$ once we assume that $A^{+}$has positive measure, i.e., $Q^{+} \not \equiv 0$. Our main result reads as follows.

Theorem 4.1. Let $p \in\left[\frac{2(N+1)}{N-1}, \frac{2 N}{N-2}\right)$ and $Q \in L^{\infty}\left(\mathbb{R}^{N}\right), Q^{+} \not \equiv 0$. Moreover assume that

$$
\mathcal{K}: L^{p^{\prime}}\left(\mathbb{R}^{N}\right) \rightarrow L^{p}\left(\mathbb{R}^{N}\right) \text { is compact and } \int_{\mathbb{R}^{N}} \psi \mathcal{K} \psi d x \geq 0 \text { for all } \psi \in L^{p^{\prime}}\left(A_{-}\right) .
$$

Then for almost all $\lambda>\lambda_{0}:=\left(2 \beta \alpha^{-1}\right)^{p}$ there is a nontrivial strong solution $u \in W^{2, q}\left(\mathbb{R}^{N}\right) \cap$ $\mathcal{C}^{1, \gamma}\left(\mathbb{R}^{N}\right)$ for all $q \in\left(\frac{2 N}{N-1}, \infty\right)$ and $\gamma \in(0,1)$ of

$$
-\Delta u-k^{2} u=Q_{\lambda}(x)|u|^{p-2} u \quad \text { on } \mathbb{R}^{N} .
$$

The proof relies on a combination of a saddle-point reduction and the abstract monotonicity trick by Jeanjean-Toland [39], which provides bounded Palais-Smale sequences (only) for almost all $\lambda>\lambda_{0}$. It would clearly be desirable to extend our result to all $\lambda>\lambda_{0}$, but related a priori bounds seem to be out of reach. Notice also that [52, Theorem 1.4] suggests the existence of nontrivial solutions also for small $\lambda>0$, possibly assuming the set $\{Q>0\}$ to be small enough and following a different variational approach. Let us point out that $\lambda_{0}$ is small provided that the number $\beta$, which is the same as the operator norm $\left\|\mathbb{1}_{A_{+}} \mathcal{K}\left(\mathbb{1}_{A_{-}}\right)\right\|_{p^{\prime} \rightarrow p}$, is small compared to $\alpha>0$. In the case $p>\frac{2(N+1)}{N-1}$ this can be achieved by considering coefficient functions $Q$ such that $\operatorname{dist}\left(A_{-}, A_{+}\right)$is large enough, see [Y1, Lemma 2.6].

In the following Corollary, we show that the abstract conditions 4.8 hold for a reasonable class of sign-changing functions $Q \in L^{\infty}\left(\mathbb{R}^{N}\right)$. If for instance $Q$ vanishes at infinity, then [31, Lemma 4.1(ii)] applied to $|Q|$ implies that $\mathcal{K}: L^{p^{\prime}}\left(\mathbb{R}^{N}\right) \rightarrow L^{p}\left(\mathbb{R}^{N}\right)$ is compact. It is less immediate to verify the non-negativity assumption on the bilinear form 4.8). From [15, Corollary 5.4] we infer that this condition holds for measurable sets $A_{-}$with small enough diameter. To be more precise, if $y_{\frac{N-2}{2}}$ denotes the first (positive) zero of the Bessel function $Y_{\frac{N-2}{2}}$, then the condition $\operatorname{diam}\left(A_{-}\right)^{2} \leq k^{-1} y_{\frac{N-2}{2}}$ is sufficient. To put this condition into perspective, note that for $N=3$ we have $Y_{\frac{1}{2}}(t)=-\sqrt{\frac{2}{\pi t}} \cos t$, thus $y_{1 / 2}=\pi / 2$ and $y_{\frac{N-2}{2}}>y_{1 / 2}$ for $N>3$ (see [1] Section 9.5]). We thus conclude as follows.

Corollary 4.2. Assume $p \in\left[\frac{2(N+1)}{N-1}, \frac{2 N}{N-2}\right)$ and $Q \in L^{\infty}\left(\mathbb{R}^{N}\right), Q^{+} \not \equiv 0$. Moreover assume 


$$
\lim _{R \rightarrow \infty} \operatorname{esssup}_{|x| \geq R}|Q(x)|=0 \quad \text { and } \quad \operatorname{diam}\left(A_{-}\right) \leq k^{-1} y_{\frac{N-2}{2}} .
$$

Then for almost all $\lambda>\lambda_{0}:=\left(2 \beta \alpha^{-1}\right)^{p}$ there is a nontrivial strong solution $u \in W^{2, q}\left(\mathbb{R}^{N}\right) \cap$ $\mathcal{C}^{1, \gamma}\left(\mathbb{R}^{N}\right)$ for all $q \in\left(\frac{2 N}{N-1}, \infty\right)$ and $\gamma \in(0,1)$ of $(4.9)$.

The regularity results in Theorem 4.1 and Corollary 4.2 are direct consequences of $\mathbf{3 1}$, Lemma 4.3] and of the iteration procedure from Step 3 in the proof of [51, Theorem 1]. Notice that [51, Theorem 1] provides solutions to far more general Nonlinear Helmholtz equations than 4.1. regardless of whether sign-changes occur or not, but the constructed solutions are small. This result relies on a fixed point approach. Let us also mention [30] where nontrivial solutions of Nonlinear Helmholtz equations are constructed for rather general and possibly sign-changing nonlinearities vanishing identically outside some compact subset of $\mathbb{R}^{N}$. Our method is entirely different from any of these approaches.

This paper is organzied as follows: In Section 2 we introduce our basic tools and develop the dual variational framework by reformulating the indefinite Nonlinear Helmholtz equation as a coupled system of integral equations. Then we prove that nontrivial critical points of the associated energy functional $J_{\lambda}$ are indeed nontrivial solutions $u \in L^{p}\left(\mathbb{R}^{N}\right)$ of the integral equation $u=\mathbf{R}\left(Q_{\lambda}|u|^{p-2} u\right)$. This motivates the search for critical points of $J_{\lambda}$. In Section 3 we perform the saddle-point reduction of $(\varphi, \psi) \mapsto J_{\lambda}(\varphi, \psi)$ leading to a reduced function $\tilde{J}_{\lambda}$ that depends on $\varphi$ only. In Section 4 we establish the existence of bounded Palais-Smale sequences for these reduced functionals for almost all $\lambda>\lambda_{0}$. As mentioned above, this step entirely relies on the monotonicity trick by Jeanjean and Toland [39]. Finally, we combine all the auxiliary results to prove Theorem 4.1 and Corollary 4.2 in Section 5.

Let us close this introduction by fixing some notation: Throughout the paper we denote by $B_{r}(x)$ the open ball in $\mathbb{R}^{N}$ with radius $r>0$ and center at $x \in \mathbb{R}^{N}$. Moreover, we set $B_{r}=B_{r}(0)$ for any $r>0$. For $1 \leq s \leq \infty$, we abbreviate the standard norm on $L^{s}\left(\mathbb{R}^{N}\right)$ by $\|\cdot\|_{s}$. The Schwartz-class of rapidly decreasing functions on $\mathbb{R}^{N}$ is denoted by $\mathcal{S}\left(\mathbb{R}^{N}\right)$. For any $p \in(1, \infty)$ we always denote by $p^{\prime}:=\frac{p}{p-1}$ the Hölder conjugate of $p$. The indicator function of a measurable set $B \subset \mathbb{R}^{N}$ is $\mathbb{1}_{B}$. By $\operatorname{diam}(B)$ we always denote the diametere of a set. We will always use the symbols $\varphi, \psi$ to denote $L^{p^{\prime}}\left(A_{+}\right)$- and $L^{p^{\prime}}\left(A_{-}\right)$-functions that are continued trivially to the whole of $\mathbb{R}^{N}$.

\section{Dual variational formulation}

In this section we will formulate a variational framework to the equation 4.9 ). We recall from the introduction that solutions of our problem are obtained as solutions of the integral equation

$$
u=\mathbf{R}\left(Q_{\lambda}|u|^{p-2} u\right), \quad u \in L^{p}\left(\mathbb{R}^{N}\right) .
$$


where $\mathbf{R}(f)=\Psi * f$ for the function $\Psi$ introduced in 4.4 and

$$
\|\mathbf{R}(f)\|_{L^{p}\left(\mathbb{R}^{N}\right)} \leq C\|f\|_{L^{p^{\prime}\left(\mathbb{R}^{N}\right)}}
$$

for all $p \in\left[\frac{2(N+1)}{N-1}, \frac{2 N}{N-2}\right]$ and some constant $C>0$.

To obtain the dual variational formualation of (4.11) we introduce $v:=\mathbb{1}_{A_{+}} u$ and $w:=\mathbb{1}_{A_{-}} u$. Then (4.11) is equivalent to the system

$$
\begin{aligned}
v & =\lambda \mathbb{1}_{A_{+}} \mathbf{R}\left[Q_{+}|v|^{p-2} v\right]-\mathbb{1}_{A_{+}} \mathbf{R}\left[Q_{-}|w|^{p-2} w\right], \\
w & =\lambda \mathbb{1}_{A_{-}} \mathbf{R}\left[Q_{+}|v|^{p-2} v\right]-\mathbb{1}_{A_{-}} \mathbf{R}\left[Q_{-}|w|^{p-2} w\right] .
\end{aligned}
$$

Setting

$$
\varphi:=\lambda Q_{+}^{1 / p^{\prime}}|v|^{p-2} v \in L^{p^{\prime}}\left(A_{+}\right), \quad \psi:=Q_{-}^{1 / p^{\prime}}|w|^{p-2} w \in L^{p^{\prime}}\left(A_{-}\right)
$$

we deduce

Similarly

$$
\begin{aligned}
\lambda^{1-p^{\prime}}|\varphi|^{p^{\prime-2}} \varphi & =Q_{+}^{1 / p} v \\
& =\lambda Q_{+}^{1 / p} \mathbf{R}\left[Q_{+}|v|^{p-2} v\right]-Q_{+}^{1 / p} \mathbf{R}\left[Q_{-}|w|^{p-2} w\right] \\
& =Q_{+}^{1 / p} \mathbf{R}\left[Q_{+}^{1 / p} \varphi\right]-Q_{+}^{1 / p} \mathbf{R}\left[Q_{-}^{1 / p} \psi\right] \\
& =Q_{+}^{1 / p} \mathbf{R}\left[|Q|^{1 / p}(\varphi-\psi)\right] .
\end{aligned}
$$

In terms of the Birman-Schwinger operator $\mathcal{K}: f \mapsto|Q|^{\frac{1}{p}} \mathbf{R}\left(|Q|^{\frac{1}{p}} f\right)$ introduced above this can be reformulated as

$$
\begin{aligned}
\lambda^{1-p^{\prime}}|\varphi|^{p^{\prime}-2} \varphi & =\mathbb{1}_{A_{+}} \mathcal{K}(\varphi-\psi), \\
|\psi|^{p^{\prime}-2} \psi & =\mathbb{1}_{A_{-}} \mathcal{K}(\varphi-\psi)
\end{aligned}
$$

and therefore carries a variational structure through the (dual) energy functional $J_{\lambda}$ on $L^{p^{\prime}}\left(A_{+}\right) \times$ $L^{p^{\prime}}\left(A_{-}\right)$given by

$$
J_{\lambda}(\varphi, \psi):=\frac{\lambda^{1-p^{\prime}}}{p^{\prime}}\|\varphi\|_{p^{\prime}}^{p^{\prime}}-\frac{1}{p^{\prime}}\|\psi\|_{p^{\prime}}^{p^{\prime}}-\frac{1}{2} \int_{\mathbb{R}^{N}}(\varphi-\psi) \mathcal{K}(\varphi-\psi) d x
$$

This functional is of class $\mathcal{C}^{1}$ with

$$
\begin{array}{ll}
\partial_{1} J_{\lambda}(\varphi, \psi)\left[h_{1}\right]=\int_{\mathbb{R}^{N}}\left(\lambda^{1-p^{\prime}}|\varphi|^{p^{\prime}-2} \varphi-\mathcal{K}(\varphi-\psi)\right) h_{1} d x, & h_{1} \in L^{p^{\prime}}\left(A_{+}\right) \\
\partial_{2} J_{\lambda}(\varphi, \psi)\left[h_{2}\right]=\int_{\mathbb{R}^{N}}\left(-|\psi|^{p^{\prime}-2} \psi-\mathcal{K}(\varphi-\psi)\right) h_{2} d x, & h_{2} \in L^{p^{\prime}}\left(A_{-}\right) .
\end{array}
$$

Here $\partial_{1}, \partial_{2}$ stand for the partial derivatives with respect to $\varphi$ and $\psi$. For this reason we will look for critical points of $J_{\lambda}$. These solve the integral equation 4.11). Thus by the regularity results [31, Lemma 4.3] and [51, p.13] these are indeed strong solutions to our original problem (4.9). 
Proposition 4.3. Let $(\varphi, \psi) \in L^{p^{\prime}}\left(A_{+}\right) \times L^{p^{\prime}}\left(A_{-}\right) \backslash\{(0,0)\}$ be a critical point of $J_{\lambda}$ where $\lambda>0$. Then

$$
u:=\mathbf{R}\left(|Q|^{\frac{1}{p}}(\varphi-\psi)\right) \in L^{p}\left(\mathbb{R}^{N}\right)
$$

is a nontrivial solution of 4.11.

Proof. Let $(\varphi, \psi) \in L^{p^{\prime}}\left(A_{+}\right) \times L^{p^{\prime}}\left(A_{-}\right) \backslash\{(0,0)\}$ be a critical point of $J_{\lambda}$. Thus we have

$$
\lambda^{1-p^{\prime}}|\varphi|^{p^{\prime}-2} \varphi=\mathbb{1}_{A_{+}} \mathcal{K}(\varphi-\psi), \quad|\psi|^{p^{\prime}-2} \psi=\mathbb{1}_{A_{-}} \mathcal{K}(\varphi-\psi)
$$

as well as

$$
\begin{aligned}
Q_{\lambda}|u|^{p-2} u & =\left(\lambda \mathbb{1}_{A_{+}}-\mathbb{1}_{A_{-}}\right)|Q||u|^{p-2} u \\
& =\left.\left.\left(\lambda \mathbb{1}_{A_{+}}-\mathbb{1}_{A_{-}}\right)|Q|^{\frac{1}{p}} \cdot|| Q\right|^{\frac{1}{p}} u\right|^{p-2} \cdot|Q|^{\frac{1}{p}} u \\
& =\left.\left.\left(\lambda \mathbb{1}_{A_{+}}-\mathbb{1}_{A_{-}}\right)|Q|^{\frac{1}{p}} \cdot||\right|^{\frac{1}{p}} \mathbf{R}\left[|Q|^{1 / p}(\varphi-\psi)\right]\right|^{p-2} \cdot|Q|^{\frac{1}{p}} \mathbf{R}\left[|Q|^{1 / p}(\varphi-\psi)\right] \\
& =\left(\lambda \mathbb{1}_{A_{+}}-\mathbb{1}_{A_{-}}\right)|Q|^{\frac{1}{p}} \cdot|\mathcal{K}[\varphi-\psi]|^{p-2} \cdot \mathcal{K}[\varphi-\psi] \\
& =\left.\left(\lambda \mathbb{1}_{A_{+}}-\mathbb{1}_{A_{-}}\right)|Q|^{\frac{1}{p}} \cdot\left|\lambda^{1-p^{\prime}}\right| \varphi\right|^{p^{\prime}-2} \varphi+\left.|\psi|^{p^{\prime}-2} \psi\right|^{p-2} \cdot\left(\lambda^{1-p^{\prime}}|\varphi|^{p^{\prime}-2} \varphi+|\psi|^{p^{\prime}-2} \psi\right) \\
& =\left(\lambda \mathbb{1}_{A_{+}}-\mathbb{1}_{A_{-}}\right)|Q|^{\frac{1}{p}} \cdot\left(\lambda^{\left(1-p^{\prime}\right)(p-1)} \varphi+\psi\right) \\
& =|Q|^{\frac{1}{p}} \cdot(\varphi-\psi) .
\end{aligned}
$$

Applying $\mathbf{R}$ then gives $\mathbf{R}\left(Q_{\lambda}|u|^{p-2} u\right)=\mathbf{R}\left(|Q|^{\frac{1}{p}}(\varphi-\psi)\right)=u$. Hence $u$ solves 4.11.

So we conclude that it remains to find nontrivial critical points of the functionals $J_{\lambda}$ for as many $\lambda>0$ as possible. This will be achieved with the Mountain Pass Theorem for families of $\mathcal{C}^{1}$-functionals by Jeanjean and Toland [39].

\section{Saddle-point reduction}

In this section we perform the saddle-point reduction of $J_{\lambda}$ with respect to the $\psi$-variable. To this end, we prove that for any fixed $\varphi \in L^{p^{\prime}}\left(A_{+}\right)$the functional $\psi \mapsto J_{\lambda}(\varphi, \psi)$ attains its maximum at some uniquely defined function in $L^{p^{\prime}}\left(A_{-}\right)$that we will call $Z(\varphi)$ in the following. We shall see that the positivity assumption $\int_{\mathbb{R}^{N}} \psi \mathcal{K} \psi d x \geq 0$ for all $\psi \in L^{p^{\prime}}\left(A_{-}\right)$ensures that the functional $\psi \mapsto J_{\lambda}(\varphi, \psi)$ is strictly concave so that the global maximization with respect to $\psi$ is the only reasonable approach to perform a saddle point reduction. We introduce the reduced functional $\tilde{J}_{\lambda}: L^{p^{\prime}}\left(A_{+}\right) \rightarrow \mathbb{R}$ via

$$
\tilde{J}_{\lambda}(\varphi):=\sup _{\psi \in L^{p^{\prime}}\left(A_{-}\right)} J_{\lambda}(\varphi, \psi) .
$$

Proposition 4.4. Assume that $\mathcal{K}: L^{p^{\prime}}\left(\mathbb{R}^{N}\right) \rightarrow L^{p}\left(\mathbb{R}^{N}\right)$ is compact and that $\int_{A_{-}} \psi \mathcal{K} \psi d x \geq 0$ for all $\psi \in L^{p^{\prime}}\left(A_{-}\right)$. Then for every $\varphi \in L^{p^{\prime}}\left(A_{+}\right)$there exists a unique $Z(\varphi) \in L^{p^{\prime}}\left(A_{-}\right)$such 
that for all $\lambda>0$ we have

$$
\tilde{J}_{\lambda}(\varphi)=J_{\lambda}(\varphi, Z(\varphi))
$$

Moreover:

(i) For any $\varphi \in L^{p^{\prime}}\left(A_{+}\right)$the corresponding maximizer $Z(\varphi)$ satisfies

$$
\|Z(\varphi)\|_{p^{\prime}} \leq\left(p^{\prime} \beta\|\varphi\|_{p^{\prime}}\right)^{\frac{1}{p^{\prime}-1}}
$$

where $\beta$ is defined in 4.7).

(ii) The map $Z: L^{p^{\prime}}\left(A_{+}\right) \rightarrow L^{p^{\prime}}\left(A_{-}\right)$is continuous.

(iii) The reduced functional $\tilde{J}_{\lambda}: L^{p^{\prime}}\left(A_{+}\right) \rightarrow \mathbb{R}$ is of class $\mathcal{C}^{1}$ with derivative

$$
\tilde{J}_{\lambda}^{\prime}[h]=\partial_{1} J_{\lambda}(\varphi, Z(\varphi))[h] .
$$

Proof. We first establish the existence of a maximizer. So fix $\varphi \in L^{p^{\prime}}\left(A_{+}\right)$and consider a maximizing sequence $\left(\psi_{n}\right)_{n} \subset L^{p^{\prime}}\left(A_{-}\right)$. Using $J_{\lambda}(\varphi, 0) \leq \sup _{\psi \in L^{p^{\prime}}\left(A_{-}\right)} J_{\lambda}(\varphi, \psi)=J_{\lambda}\left(\varphi, \psi_{n}\right)+o(1)$ as $n \rightarrow \infty$ we obtain

$$
o(1) \leq-\frac{1}{p^{\prime}}\left\|\psi_{n}\right\|_{p^{\prime}}^{p^{\prime}}+\int_{\mathbb{R}^{N}} \varphi \mathcal{K} \psi_{n}-\frac{1}{2} \int_{\mathbb{R}^{N}} \psi_{n} \mathcal{K} \psi_{n} \leq-\frac{1}{p^{\prime}}\left\|\psi_{n}\right\|_{p^{\prime}}^{p^{\prime}}+\beta\|\varphi\|_{p^{\prime}}\left\|\psi_{n}\right\|_{p^{\prime}} \quad(n \rightarrow \infty) .
$$

Here we used the nonnegativity assumption on $\mathcal{K}$ as well as 4.12. Hence,

$$
\left\|\psi_{n}\right\|_{p^{\prime}} \leq\left(p^{\prime} \beta\|\varphi\|\right)^{\frac{1}{p^{\prime}-1}}+o(1) \quad(n \rightarrow \infty),
$$

so $\left(\psi_{n}\right)_{n}$ is bounded. Passing to a subsequence we find $\psi^{*} \in L^{p^{\prime}}\left(A_{-}\right)$such that $\psi_{n} \rightarrow \psi^{*}$ in $L^{p^{\prime}}\left(A_{-}\right)$as $n \rightarrow \infty$. Using the compactness of $\mathcal{K}$ and the weak lower semicontinuity of the norm we find

$$
\begin{aligned}
& \sup _{\psi \in L^{p^{\prime}}\left(A_{-}\right)} J_{\lambda}(\varphi, \psi) \\
& =\frac{\lambda^{1-p^{\prime}}}{p^{\prime}}\|\varphi\|_{p^{\prime}}^{p^{\prime}}-\frac{1}{p^{\prime}}\left\|\psi_{n}\right\|_{p^{\prime}}^{p^{\prime}}-\frac{1}{2} \int_{\mathbb{R}^{N}} \varphi \mathcal{K} \varphi d x+\int_{\mathbb{R}^{N}} \varphi \mathcal{K} \psi_{n} d x-\frac{1}{2} \int_{\mathbb{R}^{N}} \psi_{n} \mathcal{K} \psi_{n} d x+o(1) \\
& =\frac{\lambda^{1-p^{\prime}}}{p^{\prime}}\|\varphi\|_{p^{\prime}}^{p^{\prime}}-\frac{1}{p^{\prime}}\left\|\psi_{n}\right\|_{p^{\prime}}^{p^{\prime}}-\frac{1}{2} \int_{\mathbb{R}^{N}} \varphi \mathcal{K} \varphi d x+\int_{\mathbb{R}^{N}} \varphi \mathcal{K} \psi^{*} d x-\frac{1}{2} \int_{\mathbb{R}^{N}} \psi^{*} \mathcal{K} \psi^{*} d x+o(1) \\
& \leq \frac{\lambda^{1-p^{\prime}}}{p^{\prime}}\|\varphi\|_{p^{\prime}}^{p^{\prime}}-\frac{1}{p^{\prime}}\left\|\psi^{*}\right\|_{p^{\prime}}^{p^{\prime}}-\frac{1}{2} \int_{\mathbb{R}^{N}} \varphi \mathcal{K} \varphi d x+\int_{\mathbb{R}^{N}} \varphi \mathcal{K} \psi^{*} d x-\frac{1}{2} \int_{\mathbb{R}^{N}} \psi^{*} \mathcal{K} \psi^{*} d x+o(1) \\
& =J_{\lambda}\left(\varphi, \psi^{*}\right)+o(1) .
\end{aligned}
$$

Hence the supremum is attained at $\psi^{*}$. Since equality must hold in the above estimate we conclude $\left\|\psi_{n}\right\|_{p^{\prime}} \rightarrow\left\|\psi^{*}\right\|_{p^{\prime}}$, whence $\psi_{n} \rightarrow \psi^{*}$ in $L^{p^{\prime}}\left(A_{-}\right)$as $n \rightarrow \infty$. This shows the existence of a maximizer satisfying the estimate stated in (i). So (i) is proved once we have established the uniqueness of the maximizer. 
To this end assume that $\psi^{*}, \psi^{\dagger} \in L^{p^{\prime}}\left(A_{-}\right)$are maximizers. Then we have

$$
\begin{aligned}
0 \leq & \frac{1}{2} J_{\lambda}\left(\varphi, \psi^{*}\right)+\frac{1}{2} J_{\lambda}\left(\varphi, \psi^{\dagger}\right)-J_{\lambda}\left(\varphi, \frac{1}{2}\left(\psi^{*}+\psi^{\dagger}\right)\right) \\
= & \frac{1}{p^{\prime}}\left(\left\|\frac{\psi^{*}+\psi^{\dagger}}{2}\right\|_{p^{\prime}}^{p^{\prime}}-\frac{1}{2}\left\|\psi^{*}\right\|_{p^{\prime}}^{p^{\prime}}-\frac{1}{2}\left\|\psi^{\dagger}\right\|_{p^{\prime}}^{p^{\prime}}\right) \\
& \quad+\frac{1}{2}\left(\int_{\mathbb{R}^{N}} \frac{\psi^{*}+\psi^{\dagger}}{2} \mathcal{K}\left[\frac{\psi^{*}+\psi^{\dagger}}{2}\right] \mathrm{d} x-\frac{1}{2} \int_{\mathbb{R}^{N}} \psi^{*} \mathcal{K} \psi^{*} \mathrm{~d} x-\frac{1}{2} \int_{\mathbb{R}^{N}} \psi^{\dagger} \mathcal{K} \psi^{\dagger} \mathrm{d} x\right) \\
= & \frac{1}{p^{\prime}}\left(\left\|\frac{\psi^{*}+\psi^{\dagger}}{2}\right\|_{p^{\prime}}^{p^{\prime}}-\frac{1}{2}\left\|\psi^{*}\right\|_{p^{\prime}}^{p^{\prime}}-\frac{1}{2}\left\|\psi^{\dagger}\right\|_{p^{\prime}}^{p^{\prime}}\right)-\frac{1}{8} \int_{\mathbb{R}^{N}}\left(\psi^{*}-\psi^{\dagger}\right) \mathcal{K}\left[\psi^{*}-\psi^{\dagger}\right] \mathrm{d} x \\
\leq & \frac{1}{p^{\prime}}\left(\left\|\frac{\psi^{*}+\psi^{\dagger}}{2}\right\|_{p^{\prime}}^{p^{\prime}}-\frac{1}{2}\left\|\psi^{*}\right\|_{p^{\prime}}^{p^{\prime}}-\frac{1}{2}\left\|\psi^{\dagger}\right\|_{p^{\prime}}^{p^{\prime}}\right) \\
\leq & 0,
\end{aligned}
$$

where we have used the non-negativity condition in the second last step and the convexity of $z \mapsto|z|^{p^{\prime}}$ in the last step. So we have equality in each estimate and conclude $\psi^{*}=\psi^{\dagger}$. Note that the maximizer does not depend on $\lambda$ since the only $\lambda$-dependent term in $J_{\lambda}(\varphi, \psi)$ is $\frac{\lambda^{1-p^{\prime}}}{p^{\prime}}\|\varphi\|_{p^{\prime}}^{p^{\prime}}$, which is independent of $\psi$.

We now prove (ii), i.e., the continuity of the map $Z$ : Assume $\varphi_{n} \rightarrow \varphi$ in $L^{p^{\prime}}\left(A_{+}\right)$and let $\left(\psi_{n}\right)_{n}:=\left(Z\left(\varphi_{n}\right)\right)_{n} \subset L^{p^{\prime}}\left(A_{-}\right)$be the associated maximizers. By 4.16$)$, the sequence $\left(\psi_{n}\right)_{n}$ is bounded and after passing to a subsequence we may assume $\psi_{n} \rightarrow \psi_{0}$ in $L^{p^{\prime}}\left(A_{-}\right)$as $n \rightarrow \infty$. Arguing as above we deduce

$$
\limsup _{n \rightarrow \infty} \tilde{J}_{\lambda}\left(\varphi_{n}\right)=\limsup _{n \rightarrow \infty} J_{\lambda}\left(\varphi_{n}, \psi_{n}\right)=\limsup _{n \rightarrow \infty} J_{\lambda}\left(\varphi, \psi_{n}\right) \leq J_{\lambda}\left(\varphi, \psi_{0}\right) \leq \tilde{J}_{\lambda}(\varphi) .
$$

using weak lower semicontinuity and $\liminf _{n \rightarrow \infty}\left\|\psi_{n}\right\|_{p^{\prime}} \geq\left\|\psi_{0}\right\|_{p^{\prime}}$. On the other hand, with the special choice $\psi=Z(\varphi)$ we obtain

$$
\liminf _{n \rightarrow \infty} \tilde{J}_{\lambda}\left(\varphi_{n}\right) \geq J_{\lambda}(\varphi, \psi)=J_{\lambda}(\varphi, Z(\varphi))=\tilde{J}_{\lambda}(\varphi) .
$$

Combining both estimates gives $\left\|\psi_{n}\right\|_{p^{\prime}} \rightarrow\left\|\psi_{0}\right\|_{p^{\prime}}$ as well as $\tilde{J}_{\lambda}\left(\varphi_{n}\right) \rightarrow \tilde{J}_{\lambda}(\varphi)$ as $n \rightarrow \infty$. Thus we have equality in (4.17), 4.18). Since maximizers are unique, we obtain $\psi_{0}=Z(\varphi)$ and in particular $Z\left(\varphi_{n}\right)=\psi_{n} \rightarrow \psi_{0}=Z\left(\varphi_{0}\right)$ in $L^{p^{\prime}}\left(A_{-}\right)$as $n \rightarrow \infty$. 
We are left to prove (iii). Let $h \in L^{p^{\prime}}\left(A_{+}\right)$be arbitrary. We can estimate the difference quotients as follows:

$$
\begin{aligned}
\liminf _{\tau \rightarrow 0} \frac{\tilde{J}_{\lambda}(\varphi+\tau h)-\tilde{J}_{\lambda}(\varphi)}{\tau} & \geq \liminf _{\tau \rightarrow 0} \frac{J_{\lambda}(\varphi+\tau h, Z(\varphi))-J_{\lambda}(\varphi, Z(\varphi))}{\tau} \\
& =\liminf _{\tau \rightarrow 0} \int_{0}^{1} \partial_{1} J_{\lambda}(\varphi+\tau \sigma h, Z(\varphi))[h] \mathrm{d} \sigma \\
& =\partial_{1} J_{\lambda}(\varphi, Z(\varphi))[h], \\
\limsup _{\tau \rightarrow 0} \frac{\tilde{J}_{\lambda}(\varphi+\tau h)-\tilde{J}_{\lambda}(\varphi)}{\tau} & \leq \limsup _{\tau \rightarrow 0} \frac{J_{\lambda}(\varphi+\tau h, Z(\varphi+\tau h))-J_{\lambda}(\tilde{\varphi}, Z(\varphi+\tau h))}{\tau} \\
& =\limsup _{\tau \rightarrow 0} \int_{0}^{1} \partial_{1} J_{\lambda}\left(\varphi^{*}+\tau \sigma h, Z(\varphi+\tau h)\right)[h] \mathrm{d} \sigma \\
& =\partial_{1} J_{\lambda}(\varphi, Z(\varphi))[h] .
\end{aligned}
$$

Here we used that $Z$ is continuous and that $\partial_{1} J_{\lambda}$ is continuous, see [65, Proposition 9] for a

similar computation. We conclude that $\tilde{J}_{\lambda}$ is Gâteaux-differentiable with continuous derivative $\varphi \mapsto \partial_{1} J_{\lambda}(\varphi, Z(\varphi))[\cdot]$, see Proposition 4.4 (iii). Hence, the reduced functional $\tilde{J}_{\lambda}$ is continuously (Fréchet-)differentiable with

$$
\tilde{J}_{\lambda}^{\prime}(\varphi)[h]=\partial_{1} J_{\lambda}(\varphi, Z(\varphi))[h] \quad \forall h \in L^{p^{\prime}}\left(A_{+}\right)
$$

as claimed.

Notice that the condition $\int_{\mathbb{R}^{N}} \psi \mathcal{K} \psi \mathrm{d} x \geq 0$ is also necessary for the existence of a global maximizer of $\psi \mapsto J_{\lambda}(\varphi, \psi)$ because otherwise this functional is unbounded from above.

\section{Palais-Smale sequences for the reduced functional}

In view of the results of the previous sections, we obtain a solution to our problem by proving the existence of a nontrivial critical point of the reduced functional $\tilde{J}_{\lambda}: L^{p^{\prime}}\left(A_{+}\right) \rightarrow \mathbb{R}$ introduced in (4.14). This will be done via Mountain-pass techniques for monotone families of functionals originating from the work of Jeanjean and Toland [39].

Definition 4.5. Let $X$ be a Banach space, $M \subset \mathbb{R}$ a compact interval. Then the family $\left(I_{\nu}\right)_{\nu \in M}$ of $\mathcal{C}^{1}$-functionals on $X$ is said to have the Mountain Pass Geometry if there exist $v_{1}, v_{2} \in X$ such that for all $\nu \in M$ it holds

$$
c_{\nu}:=\inf _{\gamma \in \Gamma} \sup _{t \in[0,1]} I_{\nu}(\gamma(t))>\max \left\{I_{\nu}\left(v_{1}\right), I_{\nu}\left(v_{2}\right)\right\},
$$

where $\Gamma:=\left\{\gamma \in C([0,1], X): \gamma(0)=v_{1}, \gamma(1)=v_{2}\right\}$.

Theorem 4.6. (Jeanjean, Toland) [39, Theorem 2.1] Assume that $X$ is a Banach space, $M \subset \mathbb{R}$ a compact interval and $\left(I_{\nu}\right)_{\nu \in M}$ a family of $\mathcal{C}^{1}$-functionals on $X$ having the Mountain Pass 
Geometry. Assume further that $\left(I_{\nu}\right)_{\nu \in M}$ has the following property:

For every sequence $\left(\nu_{n}, \varphi_{n}\right) \in M \times X$ with $\nu_{n} \nearrow \nu_{*} \in M$ and with

$$
-I_{\nu_{*}}\left(\varphi_{n}\right), \quad I_{\nu_{n}}\left(\varphi_{n}\right), \quad \frac{I_{\nu_{n}}\left(\varphi_{n}\right)-I_{\nu_{*}}\left(\varphi_{n}\right)}{\nu_{*}-\nu_{n}} \quad \text { bounded from above, }
$$

the sequence $\left(\varphi_{n}\right)$ is bounded itself, and $\limsup _{n \rightarrow \infty}\left(I_{\nu_{*}}\left(\varphi_{n}\right)-I_{\nu_{n}}\left(\varphi_{n}\right)\right) \leq 0$.

Then for almost all $\nu \in M$ there is a bounded Palais-Smale sequence (BPS) for $I_{\nu}$ at the level $c_{\nu}$.

We shall apply this result to $X=L^{p^{\prime}}\left(A_{+}\right)$and the family of $\mathcal{C}^{1}$-functionals $I_{\lambda}:=\tilde{J}_{\lambda}: X \rightarrow \mathbb{R}$. We first verify the Mountain Pass Geometry for parameters $\lambda \in\left(\lambda_{0}, \infty\right)$ where $\lambda_{0}=\left(2 \beta \alpha^{-1}\right)^{p}$. Let us recall that $\alpha, \beta$ were defined as

$$
\alpha:=\max _{\substack{\|\varphi\|_{p^{\prime}}=1, \operatorname{supp}(\varphi) \subset A_{+}}} \int_{\mathbb{R}^{N}} \varphi \mathcal{K} \varphi d x, \quad \beta:=\max _{\substack{\|\varphi\|_{p^{\prime}=\|\psi\|_{p^{\prime}}=1,} \\ \operatorname{supp}(\varphi) \subset A_{+}, \operatorname{supp}(\psi) \subset A_{-}}} \int_{\mathbb{R}^{N}} \varphi \mathcal{K} \psi d x .
$$

Proposition 4.7. Let $\mathcal{K}: L^{p^{\prime}}\left(\mathbb{R}^{N}\right) \rightarrow L^{p}\left(\mathbb{R}^{N}\right)$ be compact and assume $\int_{\mathbb{R}^{N}} \psi \mathcal{K} \psi d x \geq 0$ for all $\psi \in L^{p^{\prime}}\left(A_{-}\right)$. Then, for any given compact subinterval $M \subset\left(\lambda_{0}, \infty\right)$, the family of functionals $\left(\tilde{J}_{\lambda}\right)_{\lambda \in M}$ has the Mountain Pass Geometry according to Definition 4.5 .

Proof. For $\lambda \in M$ we define $r_{\lambda}:=\left(\lambda^{p^{\prime}-1} \alpha\right)^{1 /\left(p^{\prime}-2\right)}$. Then we have

$$
\begin{aligned}
\inf _{\|\varphi\|_{p^{\prime}}=r_{\lambda}} \tilde{J}_{\lambda}(\varphi) & =\inf _{\|\varphi\|_{p^{\prime}}=r_{\lambda}} \sup _{\psi \in L^{p^{\prime}}\left(A_{-}\right)} J_{\lambda}(\varphi, \psi) \geq \inf _{\|\varphi\|_{p^{\prime}}=r_{\lambda}} J_{\lambda}(\varphi, 0) \\
& =\inf _{\|\varphi\|_{p^{\prime}=r_{\lambda}}} \frac{\lambda^{1-p^{\prime}}}{p^{\prime}}\|\varphi\|_{p^{\prime}}^{p^{\prime}}-\frac{1}{2} \int_{\mathbb{R}^{N}} \varphi \mathcal{K} \varphi \mathrm{d} x \\
& =\frac{\lambda^{1-p^{\prime}}}{p^{\prime}} r_{\lambda}^{p^{\prime}}-\frac{\alpha}{2} r_{\lambda}^{2}=\alpha\left(\frac{1}{p^{\prime}}-\frac{1}{2}\right)\left(\lambda^{p^{\prime}-1} \alpha\right)^{\frac{2}{p^{\prime}-2}} \\
& >0 .
\end{aligned}
$$

On the other hand, we have

$$
\tilde{J}_{\lambda}(0)=\sup _{\psi \in L^{p^{\prime}\left(A_{-}\right)}} J_{\lambda}(0, \psi)=\sup _{\psi \in L^{p^{\prime}}\left(A_{-}\right)}\left[-\frac{1}{p^{\prime}}\|\psi\|_{p^{\prime}}^{p^{\prime}}-\frac{1}{2} \int_{\mathbb{R}^{N}} \psi \mathcal{K} \psi \mathrm{d} x\right]=0 .
$$

According to Definition 4.5 it therefore remains to find some $\varphi^{*} \in L^{p^{\prime}}\left(A_{+}\right)$with $\left\|\varphi^{*}\right\|_{p^{\prime}} \geq r_{\lambda_{0}}$ such that $\tilde{J}_{\lambda_{0}}\left(\varphi^{*}\right) \leq 0$ holds. Notice that in this case we actually have $\tilde{J}_{\lambda}\left(\varphi^{*}\right)<\tilde{J}_{\lambda_{0}}\left(\varphi^{*}\right) \leq 0$ 
for all $\lambda \in M \subset\left(\lambda_{0}, \infty\right)$. To achieve this we estimate $\tilde{J}_{\lambda_{0}}$ from above as follows

$$
\begin{aligned}
\tilde{J}_{\lambda_{0}}(\varphi) & =J_{\lambda_{0}}(\varphi, 0)-\frac{1}{p^{\prime}}\|Z(\varphi)\|_{p^{\prime}}^{p^{\prime}}+\int_{\mathbb{R}^{N}} \varphi \mathcal{K}(Z(\varphi))-\frac{1}{2} \int_{\mathbb{R}^{N}} Z(\varphi) \mathcal{K}(Z(\varphi)) \\
& \leq J_{\lambda_{0}}(\varphi, 0)-\frac{1}{p^{\prime}}\|Z(\varphi)\|_{p^{\prime}}^{p^{\prime}}+\beta\|\varphi\|_{p^{\prime}}\|Z(\varphi)\|_{p^{\prime}} \\
& \leq J_{\lambda_{0}}(\varphi, 0)+\frac{\beta^{p}}{p}\|\varphi\|_{p^{\prime}}^{p}
\end{aligned}
$$

where we have used that $\max _{c \geq 0}\left(-\frac{c^{p^{\prime}}}{p^{\prime}}+\beta\|\varphi\|_{p^{\prime}}^{p^{\prime}} c\right)=\frac{\beta^{p}}{p}\|\varphi\|_{p^{\prime}}^{p}$. We choose $\varphi^{*}=r_{0} \varphi_{0}$ where the function $\varphi_{0} \in L^{p^{\prime}}\left(A_{+}\right),\left\|\varphi_{0}\right\|_{p^{\prime}}=1$ attains the maximum $\alpha=\int_{\mathbb{R}^{N}} \varphi_{0} \mathcal{K} \varphi_{0} \mathrm{~d} x>0$. Then the choice $R:=\left(\frac{1}{2} \alpha \beta^{-p}\right)^{1 /(p-2)}$ yields after some computations $\left(\right.$ recall $\left.\lambda_{0}=\left(2 \beta \alpha^{-1}\right)^{p}\right)$

$$
\left\|\varphi^{*}\right\|_{p^{\prime}}=R=\left(\frac{1}{2} \alpha \beta^{-p}\right)^{\frac{1}{p-2}} \geq\left(\lambda_{0}^{p^{\prime}-1} \alpha\right)^{\frac{1}{p^{\prime}-2}}>\left(\lambda^{p^{\prime}-1} \alpha\right)^{\frac{1}{p^{\prime}-2}}=r_{\lambda} \quad \text { for all } \lambda \in M .
$$

Using again the explicit formulas for $R, \lambda_{0}$ we find

$$
\begin{aligned}
\tilde{J}_{\lambda_{0}}\left(R \varphi_{0}\right) & \leq J_{\lambda_{0}}\left(R \varphi_{0}\right)+\frac{\beta^{p} R^{p}}{p} \\
& =\frac{\lambda_{0}^{1-p^{\prime}}}{p^{\prime}} R^{p^{\prime}}-\frac{\alpha}{2} R^{2}+\frac{\beta^{p}}{p} R^{p} \\
& =\frac{R^{p^{\prime}}}{p^{\prime}} \cdot\left(\lambda_{0}^{1-p^{\prime}}-\frac{p \alpha}{2(p-1)} R^{2-p^{\prime}}+\frac{\beta^{p}}{p-1} R^{p-p^{\prime}}\right) \\
& =\frac{R^{p^{\prime}}}{p^{\prime}} \cdot\left(\lambda_{0}^{1-p^{\prime}}-\frac{1}{2} \alpha R^{2-p^{\prime}}\right) \\
& =\frac{R^{p^{\prime}}}{p^{\prime}} \cdot\left(\left(2 \beta \alpha^{-1}\right)^{-\frac{p}{p-1}}-\frac{1}{2} \alpha \cdot\left(\frac{1}{2} \alpha \beta^{-p}\right)^{\frac{1}{p-1}}\right) \\
& =0
\end{aligned}
$$

and thus the claim holds with $v_{1}=0$ and $v_{2}=\varphi^{*}=R \varphi_{0}$.

Having established the Mountain Pass Geometry of our functionals we now verify the condition $(\mathbf{H})$ in order to use Theorem 4.6

Proposition 4.8. For any compact subinterval $M \subset\left(\lambda_{0}, \infty\right)$ the family of $\mathcal{C}^{1}$-functionals $\left(\tilde{J}_{\lambda}\right)_{\lambda \in M}$ satisfies the condition $(\mathbf{H})$.

Proof. Consider a sequence $\left(\varphi_{n}, \lambda_{n}\right) \in L^{p^{\prime}}\left(A_{+}\right) \times M$ with $\lambda_{n} \nearrow \lambda_{*}$ and

$$
-\tilde{J}_{\lambda_{*}}\left(\varphi_{n}\right) \leq C, \quad \tilde{J}_{\lambda_{n}}\left(\varphi_{n}\right) \leq C, \quad \frac{\tilde{J}_{\lambda_{n}}\left(\varphi_{n}\right)-\tilde{J}_{\lambda_{*}}\left(\varphi_{n}\right)}{\nu_{*}-\nu_{n}} \leq C
$$

for all $n \in \mathbb{N}$. Then we have

$$
C \geq \frac{\tilde{J}_{\lambda_{n}}\left(\varphi_{n}\right)-\tilde{J}_{\lambda^{*}}\left(\varphi_{n}\right)}{\lambda_{*}-\lambda_{n}}=\frac{\lambda_{n}^{1-p^{\prime}}-\lambda_{*}^{1-p^{\prime}}}{\lambda_{*}-\lambda_{n}}\left\|\varphi_{n}\right\|_{p^{\prime}}^{p^{\prime}}=\left(\left(p^{\prime}-1\right) \lambda_{*}^{-p^{\prime}}+o(1)\right)\left\|\varphi_{n}\right\|_{p^{\prime}}^{p^{\prime}} \quad(n \rightarrow \infty)
$$


So we conclude that $\left(\varphi_{n}\right)$ is bounded. Furthermore, $\lambda_{n} \rightarrow \lambda^{*}>0$ gives

$$
\limsup _{n \rightarrow \infty}\left(I_{\lambda_{*}}\left(\varphi_{n}\right)-I_{\lambda_{n}}\left(\varphi_{n}\right)\right)=\limsup _{n \rightarrow \infty}\left(\lambda_{n}^{1-p^{\prime}}-\lambda_{*}^{1-p^{\prime}}\right)\left\|\varphi_{n}\right\|_{p^{\prime}}^{p^{\prime}}=0,
$$

which is all we had to show.

We thus conclude that Theorem 4.6 applies in our context and yields BPS sequences for $\tilde{J}_{\lambda}$ at the corresponding Mountain pass levels $c_{\lambda}$ for almost all $\lambda \in\left(\lambda_{0}, \infty\right)$. From the existence of BPS sequences we deduce rather easily the existence of critical points at the corresponding Mountain Pass level.

Proposition 4.9. Let $\mathcal{K}: L^{p^{\prime}}\left(\mathbb{R}^{N}\right) \rightarrow L^{p}\left(\mathbb{R}^{N}\right)$ be compact and assume $\int_{\mathbb{R}^{N}} \psi \mathcal{K} \psi d x \geq 0$ for all $\psi \in L^{p^{\prime}}\left(A_{-}\right)$. Then for all $\lambda \in\left(\lambda_{0}, \infty\right)$ every BPS sequence of $\tilde{J}_{\lambda}$ at its Mountain Pass level $c_{\lambda}$ converges to a critical point of $\tilde{J}_{\lambda}$ at the level $c_{\lambda}$.

Proof. Let $\left(\varphi_{j}\right)_{j}$ in $L^{p^{\prime}}\left(A_{+}\right)$be a BPS sequence for $\tilde{J}_{\lambda}$, i.e., $\tilde{J}_{\lambda}\left(\varphi_{j}\right) \rightarrow c>0$ and $\tilde{J}_{\lambda}^{\prime}\left(\varphi_{j}\right) \rightarrow 0$. We may thus assume w.l.o.g. $\varphi_{j} \rightarrow \varphi^{*}$. Moreover, Proposition 4.4 (i) implies the boundedness of $\left(\psi_{j}\right)_{j}:=\left(Z\left(\varphi_{j}\right)\right)_{j}$ and hence w.l.o.g. also weak convergence. For all $h \in L^{p^{\prime}}\left(A_{+}\right)$we then have, in view of the formula for $\tilde{J}_{\lambda}^{\prime}$ from Proposition 4.4 (iii),

$$
\begin{aligned}
& \left.\left|\int_{\mathbb{R}^{N}}\right| \varphi_{j}\right|^{p^{\prime}-2} \varphi_{j} h-\left|\varphi_{k}\right|^{p^{\prime}-2} \varphi_{k} h \mathrm{~d} x \mid \\
& \quad=\left|\tilde{J}_{\lambda}^{\prime}\left(\varphi_{j}\right) h-\tilde{J}^{\prime}\left(\varphi_{k}\right) h+\int_{\mathbb{R}^{N}} h \mathcal{K}\left[\varphi_{j}-\varphi_{k}\right] \mathrm{d} x-\int_{\mathbb{R}^{N}} h \mathcal{K}\left[\psi_{j}-\psi_{k}\right] \mathrm{d} x\right| \\
& \quad \leq\|h\|_{p^{\prime}} \cdot\left[\left\|\tilde{J}_{\lambda}^{\prime}\left(\varphi_{j}\right)\right\|+\left\|\tilde{J}_{\lambda}^{\prime}\left(\varphi_{k}\right)\right\|+\left\|\mathcal{K}\left[\varphi_{j}-\varphi_{k}\right]\right\|_{p}+\left\|\mathcal{K}\left[\psi_{j}-\psi_{k}\right]\right\|_{p}\right] \\
& \quad=\|h\|_{p^{\prime}} \cdot o(1) \quad(j, k \rightarrow \infty) .
\end{aligned}
$$

We infer that $\left(\left|\varphi_{j}\right|^{p^{\prime}-2} \varphi_{j}\right)_{j}$ converges strongly in $L^{p}\left(A_{+}\right)$. By uniqueness of weak limits, we infer $\left|\varphi_{j}\right|^{p^{\prime}-2} \varphi_{j} \rightarrow\left|\varphi^{*}\right|^{p^{\prime}-2} \varphi^{*}$ strongly in $L^{p}\left(A_{+}\right)$and hence in particular $\left\|\varphi_{j}\right\|_{p^{\prime}} \rightarrow\left\|\varphi^{*}\right\|_{p^{\prime}}$. This finally implies $\varphi_{j} \rightarrow \varphi^{*}$ strongly in $L^{p^{\prime}}\left(A_{+}\right)$. A standard computation finally shows $\tilde{J}_{\lambda}\left(\varphi^{*}\right)=c_{\lambda}$ as well as $\tilde{J}_{\lambda}^{\prime}\left(\varphi^{*}\right)=0$.

\section{Proof of Theorem 4.1 and Corollary 4.2}

We finally combine all auxiliary results to prove Theorem 4.1 .

Proof of Theorem 4.1. From Proposition 4.3 and Proposition 4.4 we infer that for almost all $\lambda \in\left(\lambda_{0}, \infty\right)$ a nontrivial solution $u \in L^{p}\left(\mathbb{R}^{N}\right)$ of the nonlinear Helmholtz equation 4.9. is found once we have proved the existence of nontrivial critical points of the reduced functional $\tilde{J}_{\lambda}$ for almost all $\lambda \in M$ where $M$ is an arbitrary compact subinterval of $\left(\lambda_{0}, \infty\right)$. From Proposition 4.7 we infer that the family $\left(\tilde{J}_{\lambda}\right)_{\lambda \in M}$ has the Mountain Pass Geometry. Moreover, by Proposition 4.8 , condition $(\mathbf{H})$ holds. So Theorem 4.6 yields for almost all $\lambda \in M$ a BPS sequence for $\tilde{J}_{\lambda}$ at the corresponding Mountain Pass level. By Proposition 4.9 each of these 
BPS sequences converges to a critical point $\varphi_{\lambda}$ of $\tilde{J}_{\lambda}$ at the Mountain Pass level. Since this critical point is necessarily nontrivial, we have thus obtained the desired claim for $L^{p}\left(\mathbb{R}^{N}\right)$ solutions of 4.9. From [31, Lemma 4.3] we infer that each of these solutions belongs to $W^{2, q}\left(\mathbb{R}^{N}\right) \cap \mathcal{C}^{1, \alpha}\left(\mathbb{R}^{N}\right)$ for all $p \leq q<\infty$ and $\alpha \in(0,1)$. Arguing as in Step 3 and Step 4 [51, p.13] one even obtains that these solutions belong to $W^{2, q}\left(\mathbb{R}^{N}\right)$ for all $q \in\left(\frac{2 N}{N-1}, p\right)$. In particular, these solutions are strong solutions of 4.9 , which finishes the proof.

Proof of Collorary 4.2. In order to apply Theorem 4.1 we show that 4.10 implies 4.8). In the special case $k=1$ the compactness of $\mathcal{K}$ was shown in Lemma 4.2 in [31. So the general case follows by rescaling. It therefore remains to show that $\delta:=\operatorname{diam}\left(A_{-}\right) \leq k^{-1} y_{\frac{N-2}{2}}$ implies $\int_{\mathbb{R}^{N}} \psi \mathcal{K} \psi d x \geq 0$ for all $\psi \in L^{p^{\prime}}\left(A_{-}\right)$. Due to $4.12, \mathcal{K}=|Q|^{1 / p} \mathbf{R}\left(|Q|^{1 / p} \cdot\right)$ and $Q \in L^{\infty}\left(\mathbb{R}^{N}\right)$ it suffices to prove

$$
\int_{\mathbb{R}^{N}} \psi \mathbf{R} \psi d x \geq 0, \quad \text { for all } \psi \in \mathcal{S}\left(A_{-}\right) .
$$

Using that $x, y \in A_{-}$implies $x-y \in B_{\delta}$ we infer from Corollary 5.4 in [15]

$$
\int_{\mathbb{R}^{N}} \psi \mathbf{R} \psi d x=\int_{\mathbb{R}^{N}} \psi\left[\mathbb{1}_{B_{\delta}} \Psi * \psi\right](x) d x \geq 0
$$

which proves 4.19 and hence the Corollary. 



\section{CHAPTER 5}

\section{Appendix}

\section{Failure of the nonvanishing-Theorem in the Stein-Tomas critical case}

In this section we will provide two different couterexamples regarding the nonvanishing Theorem 1.9 in the Stein-Tomas critical case $p=2_{*}=\frac{2(N+1)}{N-1}$. The first example is closely related to the construction in [12, pp. 231-233], that provides sharpness of the given range of exponents in the (non-selfdual) resolvent estimates stated in Theorem 1.2. The second example provides a possible connection to the results in [35] concerning maximizers of the Stein-Tomas inequality. Both examples result from an unpublished collaboration with G. Evéquoz.

First example: For $\rho \in[1, \infty)$, we consider the sets $C_{\rho}, D_{\rho}$ given by

$$
C_{\rho}=\left\{x \in \mathbb{R}^{N}: 2 \rho<x_{1}<\frac{5 \rho}{2} \text { and }\left|x_{i}\right|<\frac{\sqrt{\rho}}{2 \sqrt{N-1}}, 2 \leq i \leq N\right\}
$$

and

$$
D_{\rho}=\left\{x \in \mathbb{R}^{N}:-\frac{\rho}{2}<x_{1}<0 \text { and }\left|x_{i}\right|<\frac{\sqrt{\rho}}{2 \sqrt{N-1}}, 2 \leq i \leq N\right\} .
$$

First we note that in terms of sets we have $D_{\rho}=\left\{2 \rho e_{1}\right\}-C_{\rho}$ and therefore $\left|D_{\rho}\right|=\left|C_{\rho}\right|$. The latter is given by

$$
\left|C_{\rho}\right|=\frac{\rho}{2}\left(\frac{\sqrt{\rho}}{2 \sqrt{N-1}}\right)^{N-1} \omega_{N-1}=\omega_{N-1}(N-1)^{-\frac{N-1}{2}} 2^{-N} \rho^{\frac{N+1}{2}},
$$

where $\omega_{N-1}$ denotes the volume of the $(N-1)$-dimensional unit ball.

Let us now define functions $v_{\rho}, w_{\rho} \in L^{2+}\left(\mathbb{R}^{N}\right)$, where $2_{+}:=\left(2_{*}\right)^{\prime}=\frac{2(N+1)}{N+3}$, by

$$
v_{\rho}(x)=\rho^{-\frac{N+3}{4}} \mathbb{1}_{C_{\rho}}(x) e^{-i x_{1}} \quad \text { and } \quad w_{\rho}(x)=\rho^{-\frac{N+3}{4}} \mathbb{1}_{D_{\rho}}(x) e^{i x_{1}} .
$$

The families $\left(v_{\rho}\right)_{\rho \geq 1}$ and $\left(w_{\rho}\right)_{\rho \geq 1}$ are bounded in $L^{2+}\left(\mathbb{R}^{N}\right)$ and for any $r>0$ there holds

$$
\sup _{y \in \mathbb{R}^{N}} \int_{B_{r}(y)}\left|v_{\rho}\right|^{2+} d x=\rho^{-\frac{N+1}{2}} \sup _{y \in \mathbb{R}^{N}}\left|B_{r}(y) \cap C_{\rho}\right| \leq c_{N} \rho^{-\frac{N+1}{2}} r^{N} \omega_{N} \rightarrow 0, \text { as } \rho \rightarrow \infty,
$$

and similarly: $\sup _{y \in \mathbb{R}^{N}} \int_{B_{r}(y)}\left|w_{\rho}\right|^{2+} d x \rightarrow 0$, as $\rho \rightarrow \infty$.

We remark furthermore that $w_{\rho}(x)=e^{2 i \rho} v_{\rho}\left(2 \rho e_{1}-x\right)$, and since $\mathcal{R}$ is equivariant under translations we find

$$
\int_{\mathbb{R}^{N}} w_{\rho} \mathcal{R} w_{\rho} d x=e^{4 i \rho} \int_{\mathbb{R}^{N}} v_{\rho} \mathcal{R} v_{\rho} d x
$$


Now we distinguish the cases $\limsup _{\rho \rightarrow \infty}\left|\int_{\mathbb{R}^{N}} v_{\rho} \mathcal{R} v_{\rho} d x\right|>0$ and $\limsup _{\rho \rightarrow \infty}\left|\int_{\mathbb{R}^{N}} v_{\rho} \mathcal{R} v_{\rho} d x\right|=0$ and provide in each case a family $\left(f_{\rho}\right)_{\rho \geq 1}$ that satisfies

$$
\limsup _{\rho \rightarrow \infty}\left|\int_{\mathbb{R}^{N}} f_{\rho} \mathcal{R} f_{\rho} d x\right|>0 \text { and } \lim _{\rho \rightarrow \infty}\left[\sup _{y \in \mathbb{R}^{N}} \int_{B_{r}(y)}\left|f_{\rho}(x)\right|^{2+} d x\right]=0 \text { for any } r>0 .
$$

If $\limsup _{\rho \rightarrow \infty}\left|\int_{\mathbb{R}^{N}} v_{\rho} \mathcal{R} v_{\rho} d x\right|>0$ then the family $\left(f_{\rho}\right)_{\rho \geq 1}=\left(v_{\rho}\right)_{\rho \geq 1}$ satisfies (5.3) due to 5.1).

Let us assume that $\limsup _{\rho \rightarrow \infty}\left|\int_{\mathbb{R}^{N}} v_{\rho} \mathcal{R} v_{\rho} d x\right|=0$.

In this case, we will show that the family of functions $\left(f_{\rho}\right)_{\rho \geq 1}:=\left(v_{\rho}+w_{\rho}\right)_{\rho \geq 1}$ will satisfy 5.3 .

First let us note, that the second assertion in (5.3) is implied by (5.1) and the corresponding result for $\left(w_{\rho}\right)_{\rho \geq 1}$. Concerning the first assertion in $(5.3)$, notice that the symmetry stated in Lemma 1.30 of the quadratic form implies

$$
\int_{\mathbb{R}^{N}} w_{\rho} \mathcal{R} v_{\rho} d x=\int_{\mathbb{R}^{N}} v_{\rho} \mathcal{R} w_{\rho} d x \quad \text { for every } \rho \geq 1,
$$

and since we are assuming

$$
\limsup _{\rho \rightarrow \infty}\left|\int_{\mathbb{R}^{N}} v_{\rho} \mathcal{R} v_{\rho} d x\right|=0
$$

we find that

$$
\limsup _{\rho \rightarrow \infty}\left|\int_{\mathbb{R}^{N}}\left(v_{\rho}+w_{\rho}\right) \mathcal{R}\left(v_{\rho}+w_{\rho}\right) d x\right|=2 \limsup _{\rho \rightarrow \infty}\left|\int_{\mathbb{R}^{N}} w_{\rho} \mathcal{R} v_{\rho} d x\right|,
$$

using (5.2). Hence, to prove the first assertion in (5.3) for $\left(f_{\rho}\right)_{\rho \geq 1}:=\left(v_{\rho}+w_{\rho}\right)_{\rho \geq 1}$, it is enough to show

$$
\limsup _{\rho \rightarrow \infty}\left|\int_{\mathbb{R}^{N}} w_{\rho} \mathcal{R} v_{\rho} d x\right|>0
$$

The following approach is inspired by Börjeson [12, pp.231-233]. For this we start by considering the kernel $\Omega(x)=|x|^{\frac{1-N}{2}} e^{i|x|}, x \neq 0$ and write

$$
\begin{aligned}
\int_{\mathbb{R}^{N}} \int_{\mathbb{R}^{N}} w_{\rho}(x) \Omega(x-y) v_{\rho}(y) d y d x & =\rho^{-\frac{N+3}{2}} \int_{D_{\rho}} \int_{C_{\rho}} \frac{e^{i\left(|x-y|+\left(x_{1}-y_{1}\right)\right)}}{|x-y|^{\frac{N-1}{2}}} d y d x \\
& =\rho^{N-1} \int_{\rho^{-1} D_{\rho}} \int_{\rho^{-1} C_{\rho}} \frac{e^{i \rho\left(|x-y|+\left(x_{1}-y_{1}\right)\right)}}{|x-y|^{\frac{N-1}{2}}} d y d x .
\end{aligned}
$$

For $x \in \rho^{-1} D_{\rho}, y \in \rho^{-1} C_{\rho}$, there holds $-\frac{1}{2}<x_{1}<0,2<y_{1}<\frac{5}{2}$ and $\left|x_{i}\right|,\left|y_{i}\right|<\frac{1}{2}((N-1) \rho)^{-\frac{1}{2}}$ for all $2 \leq i \leq N$. Consequently, $\left|x_{1}-y_{1}\right|=-\left(x_{1}-y_{1}\right)>2>1-\frac{1}{4 \rho}$ for all $\rho \geq 1$ and this gives

$$
\frac{1}{2} \geq \frac{1}{2 \rho} \geq-\left|x_{1}-y_{1}\right|+\sqrt{\left|x_{1}-y_{1}\right|^{2}+\frac{1}{\rho}} \geq|x-y|+\left(x_{1}-y_{1}\right) \geq 0 .
$$


Plugging this estimate in the last integral above and taking real parts we obtain the uniform bound

$$
\begin{aligned}
& \operatorname{Re}\left(\int_{\mathbb{R}^{N}} \int_{\mathbb{R}^{N}} w_{\rho}(x) \Omega(x-y) v_{\rho}(y) d y d x\right) \\
& \geq \rho^{N-1} \int_{\rho^{-1} D_{\rho}} \int_{\rho^{-1} C_{\rho}} \frac{\cos \left(\frac{1}{2}\right)}{2^{\frac{N-1}{2}}} d y d x \\
& =2^{-\frac{N-1}{2}} \cos (1 / 2) \rho^{N-1} \rho^{-2 N}\left|D_{\rho}\right|\left|C_{\rho}\right| \\
& =2^{\frac{-3 N+1}{2}} \cos \left(\frac{1}{2}\right) \omega_{N-1}^{2}(N-1)^{-N-1}
\end{aligned}
$$

for every $\rho \geq 1$.

Now as in the proof of [31, Proposition 2.6], we have for $x \in D_{\rho}, y \in C_{\rho}$ that

$$
\Phi(x-y)=\gamma_{N} \Omega(x-y)(1+\delta(|x-y|)),
$$

where $\gamma_{N}:=\frac{1}{2}(2 \pi)^{-\frac{N-1}{2}} e^{-i \frac{N-3}{4}}$ and where $r \mapsto \delta(r)$ is a function satisfying $\delta_{*}:=\sup _{r \geq 1} r|\delta(r)|<$ $\infty$. As a consequence,

$$
\begin{aligned}
& \left|\int_{\mathbb{R}^{N}} \int_{\mathbb{R}^{N}} w_{\rho}(x)\left(\Phi(x-y)-\gamma_{N} \Omega(x-y)\right) v_{\rho}(y) d y d x\right| \\
& \leq\left|\gamma_{N}\right| \rho^{-\frac{N+3}{2}} \int_{D_{\rho}} \int_{C_{\rho}} \frac{|\delta(x-y)|}{|x-y|^{\frac{N-1}{2}}} d y d x \\
& \leq\left|\gamma_{N}\right| \delta_{*} \rho^{-\frac{N+3}{4}} \int_{D_{\rho}} \int_{C_{\rho}}(2 \rho)^{-\frac{N+1}{2}} d y d x \\
& =\left|\gamma_{N}\right| \delta_{*} 2^{-\frac{5 N+1}{2}} \omega_{N-1}^{2}(N-1)^{1-N} \rho^{-1}
\end{aligned}
$$

for all $\rho \geq 1$. It follows

$$
\begin{aligned}
\left|\int_{\mathbb{R}^{N}} w_{\rho} \mathcal{R} v_{\rho} d x\right| & \geq\left|\gamma_{N}\right| \operatorname{Re}\left(\int_{\mathbb{R}^{N}} \int_{\mathbb{R}^{N}} w_{\rho}(x) \Omega(x-y) v_{\rho}(y) d y d x\right) \\
& \quad-\left|\int_{\mathbb{R}^{N}} \int_{\mathbb{R}^{N}} w_{\rho}(x)\left(\Phi(x-y)-\gamma_{N} \Omega(x-y)\right) v_{\rho}(y) d y d x\right| \\
& \geq\left|\gamma_{N}\right| 2^{\frac{-3 N+1}{2}} \cos \left(\frac{1}{2}\right) \omega_{N-1}^{2}(N-1)^{-N-1}-\left|\gamma_{N}\right| \delta_{*} 2^{-\frac{5 N+1}{2}} \omega_{N-1}^{2}(N-1)^{1-N} \rho^{-1} \\
& >0
\end{aligned}
$$

for $\rho$ large. Therefore (5.4) holds and we conclude that $\left(f_{\rho}\right)_{\rho \geq 1}=\left(v_{\rho}+w_{\rho}\right)_{\rho \geq 1}$ satisfies (5.3) as claimed.

Second example For the second example we introduce the anisotropic scaling

$$
d_{\rho}: \mathbb{R}^{N} \rightarrow \mathbb{R}^{N}, \quad d_{\rho}\left(x_{1}, x_{2}, \ldots, x_{N}\right)=\left(\frac{x_{1}}{\rho}, \frac{x_{2}}{\sqrt{\rho}}, \ldots, \frac{x_{N}}{\sqrt{\rho}}\right)
$$


for $\rho>0$. Based on this scaling we define

$$
f_{\rho}(x):=\rho^{-\frac{N+3}{4}}\left(\eta\left(d_{\rho}(x)\right) e^{-\mathrm{i} x_{1}}+\overline{\eta\left(d_{\rho}(x)\right)} e^{\mathrm{i} x_{1}}\right), \quad \rho \geq 1
$$

for some fixed $\eta \in \mathcal{S}\left(\mathbb{R}^{N}\right) \backslash\{0\}$ such that $\widehat{\eta}$ is supported in the set

$$
P:=\left\{\xi \in \mathbb{R}^{N}: \xi_{1}<\frac{1}{2} \sum_{j=2}^{N} \xi_{j}^{2}\right\} .
$$

We will now show that the family $\left(f_{\rho}\right)_{\rho \geq 1}$ given in $(5.5)$, which is bounded in $L^{2+}\left(\mathbb{R}^{N}\right)$ satisfies (5.3).

First, for given $y \in \mathbb{R}^{N}$ and $r>0$ we have

$$
\begin{aligned}
\int_{B_{r}(y)}\left|f_{\rho}(x)\right|^{2+} d x & \leq \rho^{-\frac{N+1}{2}} \int_{B_{r}(y)}\left|\eta\left(d_{\rho}(x)\right) e^{-i x_{1}}+\overline{\eta\left(d_{\rho}(x)\right)} e^{i x_{1}}\right|^{2_{+}} d x \\
& \leq \rho^{-\frac{N+1}{2}} 2^{2_{+}} \int_{B_{r}(y)}\left|\eta\left(d_{\rho}(x)\right)\right|^{2_{+}} d x \\
& \leq 2^{2+} \int_{B_{r / \rho}\left(d_{\rho}(y)\right)}|\eta(x)|^{2+} d x \rightarrow 0, \quad \text { as } \rho \rightarrow \infty,
\end{aligned}
$$

where in the last step we have used the inclusion

$$
\left\{x \in \mathbb{R}^{N}:\left|d_{1 / \rho}(x)-y\right|<r\right\} \subset\left\{x \in \mathbb{R}^{N}:\left|x-d_{\rho}(y)\right|<\frac{r}{\rho}\right\} .
$$

For the Fourier transform of $f_{\rho}$ we have

$$
\widehat{f}_{\rho}(\xi)=\rho^{\frac{N-1}{4}}\left[\widehat{\eta}\left(\rho\left(\xi_{1}+1\right), \sqrt{\rho} \xi_{2}, \ldots, \sqrt{\rho} \xi_{N}\right)+\widehat{\widehat{\eta}\left(\rho\left(1-\xi_{1}\right),-\sqrt{\rho} \xi_{2}, \ldots,-\sqrt{\rho} \xi_{N}\right)}\right]
$$

and since $\overline{f_{\rho}}=f_{\rho}$, we conclude that $\check{f}_{\rho}=\overline{\widehat{f}_{\rho}}$. Here $\check{f}_{\rho}$ denotes the inverse Fourier transform

$$
\check{f}_{\rho}(\xi)=(2 \pi)^{-\frac{N}{2}} \int_{\mathbb{R}^{N}} e^{\mathrm{i} x \cdot \xi} f_{\rho}(x) d x .
$$

Now by definition of the resolvent operator $\mathcal{R}$ for any Schwartz-function $u$ we have

$$
\begin{aligned}
\int_{\mathbb{R}^{N}} u \mathcal{R} u d x & =\int_{\mathbb{R}^{N}} \int_{\mathbb{R}^{N}} u(x) u(y) \Phi(x-y) d x d y=\int_{\mathbb{R}^{N}} \check{u}(\xi) \widehat{\Phi * u}(\xi) d \xi \\
& =(2 \pi)^{\frac{N}{2}}\langle\widehat{\Phi}, \check{u} \widehat{u}\rangle_{\mathcal{S}^{\prime} \times \mathcal{S}}=(2 \pi)^{\frac{N}{2}} \lim _{\varepsilon \rightarrow 0^{+}} \int_{\mathbb{R}^{N}} \frac{\check{u}(\xi) \widehat{u}(\xi)}{|\xi|^{2}-(1+\mathrm{i} \varepsilon)} d \xi .
\end{aligned}
$$


Consequently for $f_{\rho}$ we obtain

$$
\begin{aligned}
(2 \pi)^{-\frac{N}{2}} & \int_{\mathbb{R}^{N}} f_{\rho} \mathcal{R} f_{\rho} d x=\lim _{\varepsilon \rightarrow 0^{+}} \int_{\mathbb{R}^{N}} \frac{\left|\widehat{f}_{\rho}(\xi)\right|^{2}}{|\xi|^{2}-(1+\mathrm{i} \varepsilon)} d \xi \\
= & \lim _{\varepsilon \rightarrow 0^{+}} \rho^{\frac{N-1}{2}} \int_{\mathbb{R}^{N}} \frac{\left|\widehat{\eta}\left(\rho\left(\xi_{1}+1\right), \sqrt{\rho} \xi_{2}, \ldots, \sqrt{\rho} \xi_{N}\right)+\overline{\widehat{\eta}\left(\rho\left(1-\xi_{1}\right),-\sqrt{\rho} \xi_{2}, \ldots,-\sqrt{\rho} \xi_{N}\right)}\right|^{2}}{|\xi|^{2}-(1+\mathrm{i} \varepsilon)} \\
= & \lim _{\varepsilon \rightarrow 0^{+}} \rho^{\frac{N-1}{2}} \int_{\mathbb{R}^{N}} \frac{\left|\widehat{\eta}\left(\rho\left(\xi_{1}+1\right), \sqrt{\rho} \xi_{2}, \ldots, \sqrt{\rho} \xi_{N}\right)\right|^{2}+\left|\widehat{\hat{\eta}\left(\rho\left(1-\xi_{1}\right),-\sqrt{\rho} \xi_{2}, \ldots,-\sqrt{\rho} \xi_{N}\right)}\right|^{2}}{|\xi|^{2}-(1+\mathrm{i} \varepsilon)} d \xi \\
& +2 \lim _{\varepsilon \rightarrow 0^{+}} \rho^{\frac{N-1}{2}} \int_{\mathbb{R}^{N}} \frac{\widehat{\eta}\left(\rho\left(\xi_{1}+1\right), \sqrt{\rho} \xi_{2}, \ldots, \sqrt{\rho} \xi_{N}\right) \frac{\widehat{\eta}\left(\rho\left(1-\xi_{1}\right),-\sqrt{\rho} \xi_{2}, \ldots,-\sqrt{\rho} \xi_{N}\right)}{|\xi|^{2}-(1+\mathrm{i} \varepsilon)}}{|\xi|^{2}-(1+\mathrm{i} \varepsilon)} \\
= & 2 \lim _{\varepsilon \rightarrow 0^{+}} \rho^{\frac{N-1}{2}} \int_{\mathbb{R}^{N}} \frac{\left|\widehat{\eta}\left(\rho\left(\xi_{1}+1\right), \sqrt{\rho} \xi_{2}, \ldots, \sqrt{\rho} \xi_{N}\right)\right|^{2}}{\mid \xi} \\
& +2 \lim _{\varepsilon \rightarrow 0^{+}} \rho^{\frac{N-1}{2}} \int_{\mathbb{R}^{N}} \frac{\widehat{\eta}\left(\rho\left(\xi_{1}+1\right), \sqrt{\rho} \xi_{2}, \ldots, \sqrt{\rho} \xi_{N}\right) \frac{\widehat{\eta}\left(\rho\left(1-\xi_{1}\right),-\sqrt{\rho} \xi_{2}, \ldots,-\sqrt{\rho} \xi_{N}\right)}{|\xi|^{2}-(1+\mathrm{i} \varepsilon)}}{}
\end{aligned}
$$

where we have substituted $-\xi_{k} \rightarrow \xi_{k}, k=1 \ldots N$ in the first integral in the last line. Since $\widehat{\eta}$ has compact support, we can find $\rho_{0} \geq 1$ such that

$$
\widehat{\eta}\left(\rho\left(\xi_{1}+1\right), \sqrt{\rho} \xi_{2}, \ldots, \sqrt{\rho} \xi_{N}\right) \widehat{\eta}\left(\rho\left(1-\xi_{1}\right),-\sqrt{\rho} \xi_{2}, \ldots,-\sqrt{\rho} \xi_{N}\right)=0
$$

for every $\rho \geq \rho_{0}$ and all $\xi \in \mathbb{R}^{N}$. For these $\rho$ therefore we have

$$
\begin{aligned}
& (2 \pi)^{-\frac{N}{2}} \int_{\mathbb{R}^{N}} f_{\rho} \mathcal{R} f_{\rho} d x=2 \rho^{\frac{N-1}{2}} \lim _{\varepsilon \rightarrow 0^{+}} \int_{\mathbb{R}^{N}} \frac{\left|\widehat{\eta}\left(\rho\left(\xi_{1}+1\right), \sqrt{\rho} \xi_{2}, \ldots, \sqrt{\rho} \xi_{N}\right)\right|^{2}}{|\xi|^{2}-(1+\mathrm{i} \varepsilon)} d \xi \\
& \quad=2 \lim _{\varepsilon \rightarrow 0^{+}} \int_{\mathbb{R}^{N}} \frac{|\widehat{\eta}(\xi)|^{2}}{\rho\left(\left|d_{\rho}(\xi)-e_{1}\right|^{2}-(1+\mathrm{i} \varepsilon)\right)} d \xi \\
& \quad=2 \lim _{\varepsilon \rightarrow 0^{+}} \int_{\mathbb{R}^{N}} \frac{|\widehat{\eta}(\xi)|^{2}}{\xi_{1}^{2} / \rho+\sum_{j=2}^{N} \xi_{j}^{2}-2 \xi_{1}-\mathrm{i} \varepsilon} d \xi \\
& \quad=2 \int_{\mathbb{R}^{N}} \frac{|\widehat{\eta}(\xi)|^{2}}{\xi_{1}^{2} / \rho+\sum_{j=2}^{N} \xi_{j}^{2}-2 \xi_{1}} d \xi
\end{aligned}
$$

Now since $\widehat{\eta}$ is supported in $P$ we conclude

$$
\lim _{\rho \rightarrow \infty}(2 \pi)^{-\frac{N}{2}} \int_{\mathbb{R}^{N}} f_{\rho} \mathcal{R}\left(f_{\rho}\right) d x=2 \int_{\mathbb{R}^{N}} \frac{|\widehat{\eta}(\xi)|^{2}}{\sum_{j=2}^{N} \xi_{j}^{2}-2 \xi_{1}} d \xi>0
$$


as claimed.

Note that if $\widehat{\eta}$ is supported in

$$
P^{\prime}:=\left\{\xi \in \mathbb{R}^{N}: \xi_{1}>\frac{1}{2} \sum_{j=2}^{N} \xi_{j}^{2}\right\}
$$

the same result still holds, since we obtain $<0$ instead of $>0$ in the last line of the proof. Let us remark that $\left(\widehat{f}_{\rho}\right)_{\rho \geq 1}$ concentrates as $\rho \rightarrow \infty$ simultaneosly at two antipodal points $(-1,0, \ldots, 0) \in \mathbb{S}^{N-1}$ and $(1,0, \ldots, 0) \in \mathbb{S}^{N-1}$ and it would be interesting to put this in relation with the results in $\mathbf{3 5}$. 


\section{CHAPTER 6}

\section{Summary}

The dual variational framework developed in [31] for the nonlinear Helmholtz equation

$$
-\Delta u-k^{2} u=Q(x)|u|^{p-2} u, \quad x \in \mathbb{R}^{N}, k>0
$$

has opened the path for a deep study of this and related types of partial differential equations. Let us set $k=1$ for a simpler presentation. The dual approach is based on a dual reformulation of the integral equation

$$
u=\mathbf{R}\left(Q|u|^{p-2} u\right), \quad u \in L^{p}\left(\mathbb{R}^{N}\right),
$$

where $\mathbf{R}: L^{p^{\prime}}\left(\mathbb{R}^{N}\right) \rightarrow L^{p}\left(\mathbb{R}^{N}\right)$ is a (real valued) resolvent operator. Since $\mathbf{R}$ is only bounded for $p \in\left[2_{*}, 2^{*}\right]$, the approach is in general limited to this values. For $Q \in L^{\infty}\left(\mathbb{R}^{N}\right) \backslash\{0\}$ non-negative, via the transformation $v:=Q^{\frac{1}{p^{\prime}}}|u|^{p-2} u$ the above integral equation leads to

$$
|v|^{p^{\prime}-2} v=Q^{\frac{1}{p}} \mathbf{R}\left(Q^{\frac{1}{p}} v\right)
$$

whose solutions coincide with critical points of the $\mathcal{C}^{1}-$ functional $J_{Q, p}: L^{p^{\prime}}\left(\mathbb{R}^{N}\right) \rightarrow \mathbb{R}$ given by

$$
J_{Q, p}(v):=\frac{1}{p^{\prime}} \int_{\mathbb{R}^{N}}|v|^{p^{\prime}} d x-\frac{1}{2} \int_{\mathbb{R}^{N}} v \mathbf{K}_{Q, p} v d x .
$$

For $p>2$ this functional has a Mountain-Pass geometry. Compactness properties significantly depend on $p$ and decay-properties of $Q$. Under the following additional conditions, in particular the existence of nontrivial dual bound state solutions have been obtained in [31]:

1) Compact case: If $Q$ satisfies $\operatorname{esssup}_{|x| \geq R} Q(x) \rightarrow 0$ as $R \rightarrow \infty$ and $p \in\left[2_{*}, 2^{*}\right)$,

2) Periodic case: $Q$ is $\mathbb{Z}^{N}$-periodic and $p \in\left(2_{*}, 2^{*}\right)$.

In the present thesis we presented the extendability of this dual technique with regard to several aspects and obtain in particular the existence of real-valued solutions of 6.1) under these new requirements, which we will summarize in the sequel.

With regard to our first contribution [Y1], which is presented in Chapter 2, we are concerned with the Sobolev-critical case $p=2^{*}$. Mainly due to the following two reasons, the existence of real-valued solutions in [31] could initially not be carried out to the Sobolev-critical case: First, the Birman-Schwinger operator $\mathbf{K}_{Q, p}: L^{p^{\prime}}\left(\mathbb{R}^{N}\right) \rightarrow L^{p}\left(\mathbb{R}^{N}\right)$ given by $\mathbf{K}_{Q, p}(f)=Q^{\frac{1}{p}} \mathbf{R}\left(Q^{\frac{1}{p}} f\right)$ that appears in the (dual) energy functional (6.3) fails even to be locally compact for $p=2^{*}$ 
due to the lack of compactness in an underlying Sobolev-embedding. Thus, the Palais-Smale condition for $J_{Q}$ does not hold for all levels. Secondly, the non-vanishing property was not shown to be true in this case, which is crucial in order to deal with weight-functions $Q$ that do no vanish at infinity, such as periodic functions.

In our approach we overcome lack of compactness issues by restoring the Palais-Smale condition at least for certain energylevels. In particular we prove, that the Palais-Smale condition is satisfied for levels $\beta$ with

$$
0<\beta<L_{Q}^{*}:=\frac{S^{N / 2}}{N\|Q\|_{\infty}^{\frac{N-2}{2}}},
$$

where $S$ denotes the best constant in the Sobolev embedding and $L_{Q}^{*}$ is the least energy among all possible limit problems

$$
-\Delta u=Q\left(x_{0}\right)|u|^{p-2} u, \quad \in \mathbb{R}^{N}
$$

for $x_{0} \in \mathbb{R}^{N}$. A main step towards this result is the compactness of the difference $\mathbf{R}-$ $\mathbf{R}_{0}: L^{2^{+}}\left(\mathbb{R}^{N}\right) \rightarrow L_{l o c}^{2^{*}}\left(\mathbb{R}^{N}\right)$ which we were able to prove by analyzing the difference of the corresponding fundamental solutions which turn out to be central to the whole article and we think it might be of independent interest.

As a second step, we prove under an additional flatness condition on $Q$ (see (Q2) below), that the Mountain-pass level lies indeed below $L_{Q}^{*}$, which only turns out to be true for dimensions $N \geq 4$. This comes from the fact that $\Psi-\Lambda$, where $\Lambda: \mathbb{R}^{N} \backslash\{0\} \rightarrow \mathbb{R}$ is the fundamental solution associated with Laplace's equation, acts positively on sets of small diameter if $N \geq 4$ and negatively in the case $N=3$ and in fact we show that the Mountain-pass level in equal to $L_{Q}^{*}$ in this case and is not attained. From this we conclude that no dual ground state exists for $N=3$. The difference between the cases $N \geq 4$ and $N=3$ was in a related context also pointed out by Brézis and Nirenberg in [10. We discover the arising difference from another viewpoint and find it interesting that an analytical property (namely the precise values of $\Lambda$ and $\Psi$ for small arguments and the corresponding sign-property of the difference) on the dual level does not allow (dual) ground state solutions in this particular situation. We also establish the non-vanishing property for the critical case, which allows to add periodic functions in our considerations. The proof relies on a modification of a specific argument, so that the complete result can be readily formulated for $p \in\left(2_{*}, 2^{*}\right]$. Together with the given counterexamples in Chapter 5 for $p=2_{*}$, we have established a complete description of this theorem for the range $p \in\left[2 *, 2^{*}.\right]$

The main existence and nonexistence result in the context of the Sobolev-critical case reads as follows.

Theorem 6.1. Let $N \geq 3$ and consider $Q \in L^{\infty}\left(\mathbb{R}^{N}\right) \backslash\{0\}$ such that $Q \geq 0$ a.e. in $\mathbb{R}^{N}$. (1) If $N \geq 4$ and $Q$ satisfies 
(Q1) $Q=Q_{0}+Q_{\mathrm{per}}$, where $Q_{0}, Q_{\mathrm{per}} \geq 0$ are such that $Q_{\mathrm{per}}$ is periodic and $Q_{0}(x) \rightarrow 0$ as $|x| \rightarrow \infty$.

(Q2) There exists $x_{0} \in \mathbb{R}^{N}$ with $Q\left(x_{0}\right)=\max _{\mathbb{R}^{N}} Q$ and as $\left|x-x_{0}\right| \rightarrow 0$ we have

$$
Q(x)-Q\left(x_{0}\right)= \begin{cases}o\left(\left|x-x_{0}\right|^{2}\right), & \text { if } N \geq 5 \\ O\left(\left|x-x_{0}\right|^{2}\right), & \text { if } N=4\end{cases}
$$

Then, the problem

$$
-\Delta u-u=Q(x)|u|^{2^{*}} u, \quad \text { in } \mathbb{R}^{N}
$$

has a dual ground state solution.

(2) If $N=3$, then no dual ground state exists for 6.4.

Contrary to the results for noncritical $p$, we are able to allow asymptotically periodic $Q$, which for example were also considered in [29]. Regarding (Q2) we want to mention, that since $Q$ is not constant, we indeed need a condition, that controls at which rate $Q$ attains its maximal value $\|Q\|_{L^{\infty}}$. The condition (Q2), which also arises in related articles (c.f. [14) seems to go back to [25] and [24], where the optimality of this condition is shown for several critical problems.

In the subsequent article [Y2] presented in Chapter 3, we focus on the extendability of the by resolvent estimates governed range for values of $p$ by investigating in particular values $p \leq 2_{*}$. Since this condition is related to the (sharp) Stein-Tomas inequality (see Theorem 1.3), we study possible improvements of the latter and corresponding implications with regard to resolvent estimates and nonvanishing properties.

We start by establishing an improved Fourier-extension result with regard to symmetric functions and weighted norms. In particular, for $a, \alpha>0$ we define

$$
L_{\alpha}:=\left\{x=\left(x^{(N-k)}, x^{(k)}\right) \in \mathbb{R}^{N-k} \times \mathbb{R}^{k}:\left|x^{(N-k)}\right| \leq a\left|x^{(k)}\right|^{-\alpha}\right\}
$$

and set $Q_{\alpha}:=\mathbb{1}_{L_{\alpha}}$. Since $Q \in L^{\infty}\left(\mathbb{R}^{N}\right) \backslash L^{s}\left(\mathbb{R}^{N}\right)$ for all $s \in[1, \infty), Q$ has no decay so all improvements will come from the additional symmetry assumption. We consider functions that are invariant under the group action of $G_{k}:=O(N-k) \times O(k) \subset O(N)$ for $k \in\{1, \ldots, N-1\}$. Our result reads as follows.

Theorem 6.2. Let $N \geq 3, k \in\{1, \ldots, N-1\}, \alpha>0$ and consider $Q_{\alpha}=\mathbb{1}_{L_{\alpha}}$ as above. Moreover, suppose that

$$
\alpha>\frac{1}{N-1} \quad \text { if } k=1, \quad \alpha<N-1 \quad \text { if } k=N-1,
$$


and let

$$
\lambda_{N, k, \alpha}:= \begin{cases}\frac{2(N-1)-\frac{2}{\alpha}}{N-2}, & \text { if } k=1 ; \\ \max \left\{\frac{2(N-k)-\frac{2 k}{\alpha}}{N-k-1}, \frac{2 k-2 \alpha(N-k)}{k-1}\right\} & \text { if } 2 \leq k \leq N-2 ; \\ \frac{2(N-1)-2 \alpha}{N-2}, & \text { if } k=N-1 .\end{cases}
$$

Then, for there exists a constant $C=C(N, k, \alpha, a)>0$ such that for $q>\lambda_{N, k, \alpha}$ the Fourierextension estimate

$$
\left\|Q_{\alpha} \check{F}_{\sigma}\right\|_{L^{q}\left(\mathbb{R}^{N}\right)} \leq C\|F\|_{L^{2}\left(\mathbb{S}^{N-1}\right)}
$$

holds for all $G_{k}$-invariant functions $F \in L^{2}\left(\mathbb{S}^{N-1}\right)$.

A triple $(G, q, Q)$ for a closed subgroup $G \subset O(N)$, some $q \geq 1$ and a measurable function $Q$ : $\mathbb{R}^{N} \rightarrow \mathbb{C}$ is called an admissible (Fourier-)extension triple, if 6.5 holds for every $G$-invariant function $F \in L^{2}\left(\mathbb{S}^{N-1}\right)$. In order to establish the dual variational method for such values $q$ as given in Theorem 6.2, we need to study consequences for weighted resolvent operators of the form $\mathcal{R}_{Q}: f \mapsto Q[\Psi *(Q f)]$ in the case of admissible extension triples. Indeed, we are able to deduce an resolvent estimate of the form

$$
\left\|\mathcal{R}_{Q} f\right\|_{L^{p}\left(\mathbb{R}^{N}\right)} \leq C\|f\|_{L^{p^{\prime}}\left(\mathbb{R}^{N}\right)}
$$

by proving more general non-selfdual resolvent estimates in the case of an admissible extension triple $(G, q, Q)$ for any $G$-invariant Schwartz-function $f \in \mathcal{S}\left(\mathbb{R}^{N}\right)$ and $p \in\left(\frac{2 N}{N-1} \frac{2 q}{q+2}, 2^{*}\right)$. Additionally we establish a corresponding nonvanishing result for the same values of $p$. This allows in particular to obtain the existence of G-invariant solutions of (6.1) for values $2<p \leq$ $2 *$.

Theorem 6.3. Let $N \geq 3$, let $G \subset O(N)$ be a closed subgroup, and let $Q \in L_{G}^{\infty}\left(\mathbb{R}^{N}\right)$ be a real-valued non-negative function with $Q \not \equiv 0$ and with the property that

$$
\|Q\|_{L^{1}\left(B_{R}(x)\right)} \rightarrow 0 \quad \text { as }|x| \rightarrow \infty \text { for some } R>0 .
$$

Moreover, let $q \in\left[1, \frac{2(N+1)}{N-1}\right]$, and let $p \in\left(\max \left\{\frac{2 N}{N-1} \frac{2 q}{q+2}, 2\right\}, \frac{2 N}{N-2}\right)$ be such that $\left(G, q, Q^{\frac{1}{p}}\right)$ is an admissible extension triple. Then (6.1) admits a nontrivial $G$-invariant dual bound state solution.

Moreover we obtain the following alternative result in the noncritical range in the case of the special choice $G=O(N-k) \times O(k)$, that allows to drop the asymptotic assumption (6.6)

Theorem 6.4. Let $N \geq 4$, let $k \in\{2, \ldots, N-2\}$, and let $Q \in L^{\infty}\left(\mathbb{R}^{N}\right)$ be a non-negative $G_{k}$-invariant function with $Q \not \equiv 0$. Then 3.4 admits a nontrivial $G_{k}$-invariant dual bound state solution for every $p \in\left(\frac{2(N+1)}{N-1}, \frac{2 N}{N-2}\right)$. 
The third contribution [Y3 presented in Chapter 4, regards mainly the dual method itself and demonstrates how sign-changing weight-functions $Q$ can be included in the dual variational reformulation of (6.1). In particular we will also obtain the existence of dual bound state solution for 6.1 in this case.

In order to allow sign-changing $Q$ in the dual formulation, we write $Q=Q_{+}-Q_{-}$with $Q_{ \pm}=$ $|Q| \mathbb{1}_{A_{ \pm}}$and $A_{+}:=\{Q>0\}$ and $A_{-}:=\{Q<0\}$. For $\lambda>0$ we then introduce $Q_{\lambda}:=\lambda_{+} Q_{+} Q_{-}$. Considering the restrictions $\left.u\right|_{A_{ \pm}}$we then derive an system, that is equivalent to $(6.2)$ and involves (dual) variables $(\varphi, \psi) \in L^{p^{\prime}}\left(A_{+}\right) \times L^{p^{\prime}}\left(A_{-}\right)$. This system carries for $p \in\left[2_{*}, 2^{*}\right]$ a variational structure through the dual energy-functional $J_{\lambda}: L^{p^{\prime}}\left(A_{+}\right) \times L^{p^{\prime}}\left(A_{-}\right) \rightarrow \mathbb{R}$ given by

$$
J_{\lambda}(\varphi, \psi)=\frac{1}{p^{\prime}}\|\varphi\|_{p^{\prime}}^{p^{\prime}}-\frac{1}{p^{\prime}}\|\psi\|_{p^{\prime}}^{p^{\prime}}-\frac{1}{2} \int_{\mathbb{R}^{N}}(\varphi-\psi) \mathbf{K}_{Q, p}(\varphi-\psi) d x .
$$

Assuming $\mathbf{K}_{Q, p}: L^{p^{\prime}}\left(\mathbb{R}^{N}\right) \rightarrow L^{p}\left(\mathbb{R}^{N}\right)$ given by $\mathbf{K}_{Q, p}: f \mapsto|Q|^{1 / p} \mathbf{R}\left(|Q|^{1 / p} f\right)$ to be compact, the values

$$
\alpha:=\max _{\substack{\|\varphi\|_{p^{\prime}}=1, \operatorname{supp}(\varphi) \subset A_{+}}} \int_{\mathbb{R}^{N}} \varphi \mathbf{K}_{Q, p} \varphi d x, \quad \beta:=\max _{\substack{\|\varphi\|_{p^{\prime}}=\|\psi\|_{p^{\prime}}=1, \operatorname{supp}(\varphi) \subset A_{+}, \operatorname{supp}(\psi) \subset A_{-}}} \int_{\mathbb{R}^{N}} \varphi \mathbf{K}_{Q, p} \psi d x .
$$

are indeed attained with $\beta \geq 0$ and $\alpha>0$, where for the latter we additionally require $\left|A_{+}\right|>0$. Our main result in this context is the following

Theorem 6.5. Let $p \in\left[\frac{2(N+1)}{N-1}, \frac{2 N}{N-2}\right)$ and $Q \in L^{\infty}\left(\mathbb{R}^{N}\right), Q^{+} \not \equiv 0$. Moreover assume that

$$
\mathbf{K}_{Q, p}: L^{p^{\prime}}\left(\mathbb{R}^{N}\right) \rightarrow L^{p}\left(\mathbb{R}^{N}\right) \text { is compact and } \int_{\mathbb{R}^{N}} \psi \mathbf{K}_{Q, p} \psi d x \geq 0 \text { for all } \psi \in L^{p^{\prime}}\left(A_{-}\right) \text {. }
$$

Then for almost all $\lambda>\lambda_{0}:=\left(2 \beta \alpha^{-1}\right)^{p}$ there is a dual bound state solution of

$$
-\Delta u-u=Q_{\lambda}(x)|u|^{p-2} u \quad \text { on } \mathbb{R}^{N} \text {. }
$$

The proof consists of two steps. First we perform a saddle-point reduction in order to eliminate the $\psi$-variable. Thanks to the non-negativity condition in $(6.8)$, the functional $J_{\lambda}(\varphi, \cdot)$ is bounded from above and concave for any $\varphi \in L^{p^{\prime}}\left(A_{+}\right)$. This allows to prove, that for any $\varphi \in L^{p^{\prime}}\left(A_{+}\right)$there exists a unique $\psi^{*} \in L^{p^{\prime}}\left(A_{-}\right)$such that

$$
J_{\lambda}\left(\varphi, \psi^{*}\right)=\max _{\psi \in L^{p^{\prime}}\left(A_{-}\right)} J_{\lambda}(\varphi, \psi):=\tilde{J}_{\lambda}(\varphi) .
$$

The reduced functional $\tilde{J}_{\lambda}: L^{p^{\prime}}\left(A_{+}\right) \rightarrow \mathbb{R}$ has a Mountain-Pass structure for $\lambda>\lambda_{0}$ and obeys a monotonicity condition, that we will use to apply an abstract result in [39] in order to obtain the existence of bounded Palais-Smale sequences, only for almost all $\lambda>\lambda_{0}$. As mentioned, compactness of $\mathbf{K}_{Q, p}$ can for example be achieved for weight-functions $Q$ that vanish at infinity. The non-negativity property is somehow more delicate. However, using a result [15, §5] this is indeed guaranteed, if $A_{-}$is small. In particular we have the following result. 
Corollary 6.6. Assume $p \in\left[\frac{2(N+1)}{N-1}, \frac{2 N}{N-2}\right)$ and $Q \in L^{\infty}\left(\mathbb{R}^{N}\right), Q^{+} \not \equiv 0$. Moreover assume

$$
\lim _{R \rightarrow \infty} \operatorname{esssup}_{|x| \geq R}|Q|=0 \quad \text { and } \quad \operatorname{diam}\left(A_{-}\right) \leq y_{\frac{N-2}{2}},
$$

where $y_{\frac{N-2}{2}}$ is the first zero of the Bessel-function $Y_{\frac{N-2}{2}}$. Then for almost all $\lambda>\lambda_{0}:=$ $\left(2 \beta \alpha^{-1}\right)^{p}$ there is a dual bound state solution of

$$
-\Delta u-u=Q_{\lambda}|u|^{p-2} u .
$$

Clearly it would be desirable to obtain solutions for all $\lambda>\lambda_{0}$. However, boundedness of Palais-Smale sequences seem to be difficult problem, so we see our approach as a first step. We think also, that our approach may help to understand and to formulate general dual approaches for a larger class of (possibly nonlocal) problems involving sign-changing nonlinearities. 


\section{References constituting the authors dissertation thesis}

[Y1] G. Evéquoz and T. Yeşil. Dual ground state solutions for the critical nonlinear Helmholtz equation. Proceedings of the Royal Society of Edinburgh: Section A Mathematics, 150(3):1155-1186,2020

[Y2] T. Weth and T. Yeşil. Fourier-extension estimates for symmetric functions and applications to nonlinear Helmholtz-equations. Annali di matematica. Annali di Matematica Pura ed Applicata. 1-32. 2021.

[Y3] R. Mandel, D. Scheider and T. Yeşil. Dual variational methods for a nonlinear Helmholtz equation with sign-changing nonlinearity. arXiv preprint arXiv:2011.07808, 2021. Appears in Calculus of Variations and Partial Differential Equations. 



\section{References}

[1] M. Abramowitz and I. A. Stegun. Handbook of mathematical functions with formulas, graphs, and mathematical table. In US Department of Commerce. National Bureau of Standards Applied Mathematics series 55, 1965.

[2] S. Alama and Y. Li. Existence of solutions for semilinear elliptic equations with indefinite linear part. Journal of Differential Equations, 96(1):89 - 115, 1992.

[3] C. O. Alves and S. H. Soares. Singularly perturbed elliptic systems. Nonlinear Analysis: Theory, Methods and Applications, 64(1):109 - 129, 2006.

[4] A. Ambrosetti and P. H. Rabinowitz. Dual variational methods in critical point theory and applications. Journal of functional Analysis, 14(4):349-381, 1973.

[5] A. Ambrosetti and M. Struwe. A note on the problem $-\Delta u=\lambda u+|u|^{2^{*}-2} u$. Manuscripta Mathematica, 54(4):373-379, 1986.

[6] S. Axler, P. Bourdon, and R. Wade. Harmonic Function Theory, volume 137. Springer Science \& Business Media, 2013.

[7] H. Berestycki and P.-L. Lions. Nonlinear scalar field equations, I existence of a ground state. Archive for Rational Mechanics and Analysis, 82(4):313-346, 1983.

[8] H. Berestycki and P.-L. Lions. Nonlinear scalar field equations, II existence of infinitely many solutions. Archive for Rational Mechanics and Analysis, 82(4):347-375, 1983.

[9] D. Bonheure, E. Moreira dos Santos, M. Ramos, and H. Tavares. Existence and symmetry of least energy nodal solutions for Hamiltonian elliptic systems. Journal de Mathématiques Pures et Appliquées, 104(6):1075 $-1107,2015$.

[10] H. Brezis and L. Nirenberg. Positive solutions of nonlinear elliptic equations involving critical Sobolev exponents. Communications on Pure and Applied Mathematics, 36:437-477, 1983.

[11] H. Brézis, J.-M. Coron, and L. Nirenberg. Free vibrations for a nonlinear wave equation and a theorem of P. Rabinowitz. Communications on Pure and Applied Mathematics, 33(5):667-684, 1980.

[12] L. Börjeson. Estimates for the Bochner-Riesz operator with negative index. Indiana University Mathematics Journal, 35(2):225-233, 1986.

[13] T. Cazenave. Semilinear Schrödinger Equations, volume 10. American Mathematical Society, 2003.

[14] J. Chabrowski and A. Szulkin. On a semilinear Schrödinger equation with critical Sobolev exponent. Proceedings of the American Mathematical Society, 130(1):85-93, 2002.

[15] H. Chen, G. Evéquoz, and T. Weth. Complex solutions and stationary scattering for the nonlinear Helmholtz equation. arXiv:1911.09557, 2019.

[16] F. H. Clarke. Solution périodique des équations hamiltoniennes. C.R. Acad Sci. Paris Sér, 281(14):A951952, 1978.

[17] F. H. Clarke. Periodic solutions to Hamiltonian inclusions. Journal of Differential Equations, 40(1):1 - 6, 1981.

[18] F. H. Clarke and I. Ekeland. Hamiltonian trajectories having prescribed minimal period. Communications on Pure and Applied Mathematics, 33(2):103-116, 1980. 
[19] P. Clément and V. der Vorst. Differential Integral Equations, 8(6):1317-1329, 1995.

[20] D. Colton and R. Kress. Inverse acoustic and electromagnetic scattering theory. Applied mathematical sciences, 93(2), 1992.

[21] L. Cossetti and R. Mandel. A limiting absorption principle for Helmholtz systems and time-harmonic isotropic Maxwell's equations. arXiv preprint arXiv:2009.0508\%, 2020.

[22] W.-Y. Ding and W.-M. Ni. On the existence of positive entire solutions of a semilinear elliptic equation. In Analysis and Continuum Mechanics, pages 17-42. Springer, 1989.

[23] J. Dolbeault. Sobolev and Hardy-Littlewood-Sobolev inequalities: Duality and fast diffusion. arXiv preprint arXiv:1103.1145, 2011.

[24] H. Egnell. Semilinear elliptic equations involving critical Sobolev exponents. Archive for Rational Mechanics and Analysis, 104(1):27-56, 1988.

[25] J. F. Escobar. Positive solutions for some semilinear elliptic equations with critical Sobolev exponents. Communications on pure and applied mathematics, 40(5):623-657, 1987.

[26] G. Evéquoz. Existence and asymptotic behavior of standing waves of the nonlinear Helmholtz equation in the plane. Analysis, 37(2), 01 May. 2017.

[27] G. Evéquoz. A dual approach in Orlicz spaces for the nonlinear Helmholtz equation. Zeitschrift Angewandte Mathematik und Physik, 66:2995-3015, 2015.

[28] G. Evéquoz. Multiple standing waves for the nonlinear Helmholtz equation concentrating in the high frequency limit. Annali di Matematica Pura ed Applicata, 196(6):2023-2042, 2017.

[29] G. Evéquoz. On the periodic and asymptotically periodic nonlinear Helmholtz equation. Nonlinear Analysis: Theory, Methods \& Applications, 152:88-101, 2017.

[30] G. Evéquoz and T. Weth. Real solutions to the nonlinear Helmholtz equation with local nonlinearity. Arch. Ration. Mech. Anal., 211(2):359-388, 2014.

[31] G. Evéquoz and T. Weth. Dual variational methods and nonvanishing for the nonlinear Helmholtz equation. Advances in Mathematics, 280:690-728, 2015.

[32] G. Evéquoz and T. Weth. Branch continuation inside the essential spectrum for the nonlinear Schrödinger equation. Journal of Fixed Point Theory and Applications, 19(1):475-502, 2017.

[33] A. Floer and A. Weinstein. Nonspreading wave packets for the cubic Schrödinger equation with a bounded potential. Journal of Functional analysis, 69(3):397-408, 1986.

[34] D. Foschi and D. O. e Silva. Some recent progress on sharp Fourier restriction theory. Analysis Mathematica, 43(2):241-265, 2017.

[35] R. L. Frank, E. H. Lieb, and J. Sabin. Maximizers for the Stein-Tomas inequality. Geometric and Functional Analysis, 26(4):1095-1134, 2016.

[36] I. M. Gel'fand and G. E. Shilov. Generalized functions. Vol. I: Properties and operations. Translated by Eugene Saletan. Academic Press, New York-London, 1964.

[37] S. Gutiérrez. Non trivial $L^{q}$ solutions to the Ginzburg-Landau equation. Mathematische Annalen, 328(12):1-25, 2004.

[38] L. Jeanjean. Solutions in Spectral Gaps for a Nonlinear Equation of Schrödinger Type. Journal of Differential Equations, 112(1):53-80, Jan. 1994.

[39] L. Jeanjean and J. F. Toland. Bounded Palais-Smale Mountain-Pass sequences. C. R. Acad. Sci. Paris Sér. I Math., 327(1):23-28, 1998.

[40] T. Kato. Growth properties of solutions of the reduced wave equation with a variable coefficient. Communications on Pure and Applied Mathematics, 12(3):403-425, 1959.

[41] M. Keel and T. Tao. Endpoint Strichartz estimates. American Journal of Mathematics, 120(5):955-980, 1998. 
[42] C. E. Kenig. Lectures on the energy critical nonlinear wave equation, volume 122. American Mathematical Soc., 2015.

[43] C. E. Kenig, A. Ruiz, and C. D. Sogge. Uniform Sobolev inequalities and unique continuation for second order constant coefficient differential operators. Duke Mathematical Journal, 55(2):329-347, 1987.

[44] N. Lebedev and R. Silverman. Special functions and their applications. Dover Books on Mathematics. Dover Publications, 1972.

[45] E. H. Lieb. Sharp constants in the Hardy-Littlewood-Sobolev and related inequalities. In Inequalities, pages 529-554. Springer, 2002.

[46] E. H. Lieb and M. Loss. Analysis, Graduate Stud. Math., Vol. 14. In Amer. Math. Soc, 1996.

[47] P.-L. Lions. The concentration-compactness principle in the calculus of variations. The locally compact case, part 1. 1(2):109-145, 1984.

[48] P.-L. Lions. The concentration-compactness principle in the calculus of variations. The locally compact case, part 2. 1(4):223-283, 1984.

[49] P.-L. Lions. The concentration-compactness principle in the calculus of variations. The limit case, part 1. Revista matemática iberoamericana, 1(1):145-201, 1985.

[50] P.-L. Lions. The concentration-compactness principle in the calculus of variations. The limit case, part 2. Revista matemática iberoamericana, 1(2):45-121, 1985.

[51] R. Mandel. Uncountably many solutions for nonlinear Helmholtz and curl-curl equations. Advanced Nonlinear Studies, 19(3):569-593, 2019.

[52] R. Mandel, E. Montefusco, and B. Pellacci. Oscillating solutions for nonlinear Helmholtz equations. $Z$. Angew. Math. Phys., 68(6), 2017.

[53] R. Mandel and D. Scheider. Dual variational methods for a nonlinear Helmholtz system. arXiv preprint arXiv:1710.04526, 2017.

[54] R. Mandel and D. Scheider. Bifurcations of nontrivial solutions of a cubic Helmholtz system. Advances in Nonlinear Analysis, 9(1):1026-1045, 2019.

[55] J.-C. Nédélec. Acoustic and Electromagnetic Equations: Integral Representations for Harmonic Problems. Springer Science \& Business Media, 2001.

[56] F. Rellich. Über das asymptotische Verhalten der Lösungen von $\Delta u+\lambda u=0$ in unendlichen Gebieten. Jahresbericht der Deutschen Mathematiker-Vereinigung, 53:57-65, 1943.

[57] W. Rudin. Functional analysis. Inc, New York, 1991.

[58] A. Sommerfeld. Die Greensche Funktion der Schwingungslgleichung. Jahresbericht der Deutschen Mathematiker-Vereinigung, 21:309-352, 1912.

[59] E. Stein and R. Shakarchi. Functional analysis: Introduction to Further Topics in Analysis, volume 4. Princeton University Press, 2011.

[60] E. M. Stein. Oscillatory integrals in Fourier analysis. Beijing lectures in Harmonic Analysis, 112:307-355, 1986.

[61] W. A. Strauss. Nonlinear wave equations. Number 73. American Mathematical Soc., 1990.

[62] R. S. Strichartz et al. Restrictions of Fourier transforms to quadratic surfaces and decay of solutions of wave equations. Duke Mathematical Journal, 44(3):705-714, 1977.

[63] M. Struwe. Variational methods, volume 991. Springer, 2000.

[64] A. Szulkin and T. Weth. Ground state solutions for some indefinite variational problems. Journal of functional analysis, 257(12):3802-3822, 2009.

[65] A. Szulkin and T. Weth. The method of Nehari manifold. In Handbook of nonconvex analysis and applications, pages 597-632. Int. Press, Somerville, MA, 2010. 


\section{REFERENCES}

[66] T. Tao. Some recent progress on the restriction conjecture. In Fourier analysis and convexity, pages 217-243. Springer, 2004.

[67] P. Tomas et al. A restriction theorem for the Fourier transform. Bull. Amer. Math. Soc, 81(s1), 1975.

[68] G. N. Watson. A treatise on the theory of Bessel functions. Cambridge university press, 1995.

[69] M. Willem. Progress in nonlinear differential equations and their applications, 1996.

[70] M. Willem. Minimax theorems, volume 24. Springer Science \& Business Media, 1997. 